\title{
Stereochemistry of Challenging Natural Products Studied by NMR-based Methods
}

\author{
Dissertation \\ zur Erlangung des mathematisch-naturwissenschaftlichen Doktorgrades \\ "Doctor rerum naturalium" \\ der Georg-August-Universität Göttingen \\ im Promotionsprogramm Chemie \\ der Georg-August University School of Science (GAUSS)
}

vorgelegt von

Han Sun

aus Peking, V. R. China

Göttingen, 2012 
$\underline{\text { Betreuungsausschuss }}$

Prof. Dr. Christian Griesinger, NMR-basierte Strukturbiologie, Max-Planck-Institut für Biophysikalische Chemie

Prof. Dr. Philipp Vana, Makromolekulare Chemie, Institut für Physikalische Chemie

$\underline{\text { Mitglieder der Prüfungskommission }}$

Referent: Prof. Dr. Christian Griesinger, NMR-basierte Strukturbiologie, Max-PlanckInstitut für Biophysikalische Chemie

Korreferent: Prof. Dr. Philipp Vana, Makromolekulare Chemie, Institut für Physikalische Chemie

$\underline{\text { Weitere Mitglieder der Prüfungskommission: }}$

Prof. Dr. George M. Sheldrick, Strukturchemie, Institut für Anorganische Chemie

Prof. Dr. Hartmut Laatsch, Institut für Organische und Biomolekulare Chemie

Prof. Dr. Bert de Groot, Computergestützte Biomolekulare Dynamik, Max-PlanckInstitut für Biophysikalische Chemie

Dr. Claudia Höbartner, Nukleinsäurechemie, Max-Planck-Institut für Biophysikalische Chemie

Tag der mündlichen Prüfung: 
海纳百川，有容乃大

壁立千伆，无欲则刚 


\section{Abstract}

Stereochemistry is a very important subject in chemistry and life sciences, as most of the biologically active molecules, including proteins, oligonucleotides, sugars, lipids and small molecules exist in one chiral form. In addition, small molecule compounds with biological activity - drugs - with the same constitution yet different configuration can exhibit divergent biological activities. The determination of the relative configuration of stereocenters was first established by Fischer in 1891 via organic synthesis. The question of absolute configuration was not solved until the work of Bijvoet in 1951 with anomalous X-ray diffraction of single crystals. For the compounds which can neither be crystallized nor easily be converted into crystallizable derivatives, it can still be difficult to establish their stereochemistry.

Within this thesis, the development and application of Residual Dipolar Coupling (RDC)-enhanced NMR techniques for the stereochemical elucidation of organic molecules - the RDC-based approach - will be presented. Residual dipolar couplings provide valuable information about the orientation of internuclear vectors with respect to the molecular frame, which complements nuclear Overhauser effects (NOEs), scalar $J$-couplings and chemical shifts that are short-range in nature. RDCs have been used for structure determination and dynamic studies of biomolecules for decades, however the use of this anisotropic parameter for configurational and conformational studies of small molecules has yet to be fully tapped. Herein, the new developments and improvements of this RDC-based approach for the study of small molecules can be categorized as:

Conformationally flexible molecules: Both single and multiple alignment tensor approaches were employed and compared. Molecular mechanics (MM) and molecular dynamics (MD) simulations were applied to statistically sample the extensive conformational space.

Natural products with limited availability: The preparation of a slim PH-gel for $1.7 \mathrm{~mm}$ NMR tubes has been developed for reproducibility and efficiency. RDC measurements in this low volume gel system have been made using a 
number of molecules.

Large numbers of unknown stereocenters: NOE and $J$-coupling restrained MD simulations with the floating chirality method were investigated. Combined with an RDC analysis in the final step, this enables the determination of the relative stereochemistry of a large number of stereocenters in a complex natural product.

Absolute configuration: An integrated approach is herein developed. This includes diastereomer differentiation using both isotropic and anisotropic NMR data. The chiroptical properties calculated using DFT of the NMR derived ensemble from this first step are used for enantiomer differentiation by comparison to experiments.

Using these improved methods, the stereochemistry of six challenging natural products - LLG1, naphth 1, naphth 2, comp.540a, vatiparol, and fibrosterol sulfate A - and one synthetic molecule - a Michael addition product - has been successfully established. 


\section{Zusammenfassung}

Stereochemie gehört zu den wichtigsten Themen in der Chemie und den Biowissenschaften, da die meisten biologisch aktiven Moleküle, einschließlich Proteine, Oligonukleotide, Zucker, Lipide und kleine Moleküle in einer chiralen Form vorliegen. Kleine Moleküle mit biologischer Aktivität - Wirkstoffe - mit der gleichen Konstitution, aber unterschiedlicher Konfiguration, können vollständig unterschiedliche biologische Aktivität aufweisen. Die Bestimmung der relativen Konfiguration der Stereozentren wurde erstmals von Fischer im Jahr 1891 mittels organischer Synthese erreicht. Allerdings wurde die Frage nach der absoluten Konfiguration erst mit der Arbeit von Bijvoet im Jahr 1951 mit Hilfe der anomalen Röntgenbeugung an Einkristallen gelöst. Für zahlreiche Verbindungen, die weder kristallisiert, noch leicht in kristallisierbare Derivate umgewandelt werden können, kann es immer noch sehr schwierig sein, die Stereochemie zu bestimmen.

Im Rahmen dieser Dissertation werden die Entwicklung und Anwendung der auf dipolaren Restkopplungen (RDCs) beruhenden NMR-Technik für die stereochemische Aufklärung von organischen Molekülen vorgestellt. Dipolare Restkopplungen liefern wertvolle Informationen über die Orientierung internuklearer Vektoren mit Bezug auf ein molekülfixiertes Bezugssystem. Sie ergänzen Kern-Overhauser-Effekte (NOEs), skalare J-Kopplungen und chemische Verschiebungen, die von kurzreichweitiger Natur sind. Obwohl RDCs zur Strukturaufklärung und Untersuchung der Dynamik von Biomolekülen schon seit Jahrzehnten verwendet werden, fanden sie bis jetzt nur in wenigen Fällen eine Anwendung in Studien zur Konfiguration und Konformation von kleinen Molekülen. Die neuen Entwicklungen und Verbesserungen der RDC-basierten Methode für die Untersuchung von kleinen Molekülen in dieser Arbeit können in den folgenden vier Punkten zusammengefasst werden:

Konformationell flexible Moleküle: Sowohl Einzeltensor als auch Mehrfachtensor Methoden wurden angewandt und verglichen. Molekülmechanik (MM) und Moleküldynamik (MD) Simulationen wurden angewandt, um statistisch den komplexen konformationellen Raum zu erfassen. 
Naturstoffe mit begrenzter Verfügbarkeit: Die Herstellung eines dünnen PHGels für 1,7 mm NMR-Röhrchen wurde in einer reproduzierbaren und einfachen Weise vorgeschlagen. RDC Messungen in diesem Gelsystem mit kleinem Volumen wurden weiterhin in verschiedenen aktuellen Konfigurationsbestimmungen angewandt.

Eine große Anzahl von unbekannten Stereozentren: Durch NOEs und $J$ Kopplungen eingeschränkte MD-Simulationen wurden mit der floating chirality Methode untersucht und verwendet. Die Kombination mit der RDC Analyse im letzten Schritt ermöglicht die korrekte Bestimmung der relativen Konfiguration von einer Vielzahl von Stereozentren in einem komplexen Naturstoff.

Bestimmung der absoluten Konfiguration: Eine kombinierte Methode wurde hier vorgeschlagen. Die Differenzierung der Diastereomere wurde zunächst mit isotropen und anisotropen NMR-Daten erreicht und die Differenzierung der Enantiomere anschließend über eine experimentelle Validierung der DFT berechneten chiroptischen Eigenschaften von NMR abgeleiteten Ensembles.

Mit den verbesserten Methoden wurde die Stereochemie von sechs herausfordernden Naturstoffen - LLG1, Naphth 1, Naphth 2, Comp.540a, Vatiparol, und Fibrosterol sulfat A - und einem synthetischen Molekül - einem Michael-Additionsprodukt - erfolgreich durchgeführt. 


\section{Related Publications}

Part of the work presented in this $\mathrm{PhD}$ thesis is based on the following publications.

- Han Sun, Edward J. d'Auvergne, Uwe M. Reinscheid, Luiz Carlos Dias, Carlos Kleber Z. Andrade, Rafael Oliveira Rocha, and Christian Griesinger, Bijvoet in Solution Reveals Unexpected Stereoselectivity in a Michael Addition, Chemistry - A European Journal, 17(6), 1811-1817, (2011). [Sun et al., 2011a]

- Han Sun, Uwe M. Reinscheid, Emily L. Whitson, Edward J. d'Auvergne, Chris M. Ireland, Armando Navarro-Vázquez, and Christian Griesinger, Challenge of Large-Scale Motion for Residual Dipolar Coupling Based Analysis of Configuration: The Case of Fibrosterol Sulfate A, Journal of the American Chemical Society, 133(37), 14629-14636, (2011). [Sun et al., 2011b]

- Emily L. Whitson, Han Sun, Chery L. Thomas, Curtis J. Henrich, Thomas J. Sayers, James B. McMahon, Christian Griesinger, and Tawnya C. McKee, Synergistic TRAIL Sensitizers from Barleria alluaudii and Diospyros maritima, Journal of Natural Products, 75(3), 394-399, (2012). [Whitson et al., 2012]

- Hui Ming Ge*, Han Sun*, Jiang Nan, Yan Hua Qin, Huan Dou, Yan Tong, Ya Yi Hou, Christian Griesinger, and Ren Xiang Tan, Relative and Absolute Configuration of Vatiparol (1 mg), a Novel Anti-inflammatory Polyphenol, Chemistry - A European Journal, 18(17), 5213-5221, (2012). (* equally contributing authors) [Ge et al., 2012]

Additionally, there are several other works that have been published during this $\mathrm{PhD}$ thesis.

- Han Sun, Laurence Dinan, René Lafont, Apichart Suksamrarn, Christian Griesinger, Uwe Reinscheid, and Silvia Lapenna, Absolute Configuration and Docking Study of Canescensterone, a Potent Phytoecdysteroid, with Non-Lepidopteran 
Ecdysteroid Receptor Selectivity, European Journal of Organic Chemistry, 30, 5791-5799, (2010). [Sun et al., 2010]

- Fernando Hallwass, Manuel Schmidt, Han Sun, Adam Mazur, Grit Kummerlöwe, Burkhard Luy, Armando Navarro-Vázquez, Christian Griesinger, and Uwe M. Reinscheid, Residual Chemical Shift Anisotropy (RCSA): A Tool for the Analysis of the Configuration of Small Molecules, Angewandte Chemie International Edition, 50(40), 9487-9490, (2011). [Hallwass et al., 2011]

- Carlos Pérez-Balado, Han Sun, Christian Griesinger, Ángel R. de Lera, and Armando Navarro-Vázquez, Residual Dipolar Coupling Enhanced NMR Spectroscopy and Chiroptics: A Powerful Combination for the Complete Elucidation of Symmetrical Small Molecules, Chemistry - A European Journal, 17(43),11983-11986, (2011). [Pérez-Balado et al., 2011]

- Manuel Schmidt, Han Sun, Per Rogne, Gerhard K. Scriba, Christian Griesinger, Lars T. Kuhn and Uwe M. Reinscheid, Determining the Absolute Configuration of $(+)$-Mefloquine $\mathrm{HCl}$, the Side-Effect-Reducing Enantiomer of the Antimalaria Drug Lariam, Journal of the American Chemical Society, 134(6), 3080-3083, (2012). [Schmidt et al., 2012]

- Manuel Schmidt, Han Sun, Christian Griesinger and Uwe M. Reinscheid, Chiral Discrimination by Anisotropic NMR Parameters using Chiral Polyacrylamide based Gels, Magnetic Resonance in Chemistry, in press.

- Anna Lange*, Han Sun*, Jens Pilger*, Uwe Reinscheid, and Harald Gross, Predicting the Structure of Cyclic Lipopeptides by Bioinformatics: Structure Revision of Arthrofactin, ChemBioChem, in press. (* equally contributing authors) 


\section{Acknowledgements}

This thesis would not have been possible without the help and support of many people to whom I would like to express my sincere gratitude.

Firstly I would like to deeply thank my supervisor Professor Christian Griesinger who has introduced me to the world of research and to NMR spectroscopy. I am very grateful to him for the opportunity to work on this exciting project, for all the intensive discussions and continuous inspiration, for the financial support, the outstanding scientific environment, and learning numerous techniques during my PhD.

I also would like to thank Prof. Philipp Vana for accepting me as an external PhD student and many helpful discussions at my thesis committee meetings. I am grateful to Prof. George Sheldrick, Prof. Hartmut Laatsch, Prof. Bert de Groot, and Dr. Claudia Höbartner for agreeing to be members of my $\mathrm{PhD}$ thesis committee. I am grateful to Prof. Martin Suhm for the financial and scientific support through the Physical and Chemical Graduate School Göttingen (GRK 782) and Prof. Bert de Groot for giving me the opportunity to perform my research week in the framework of the GRK 782 in his group.

I would like to express my special appreciation to Prof. Uwe Reinscheid for the extensive support and guidance during these four years, for organizing many interesting projects, for always being there, and also for carefully reading this thesis.

I would like to thank sincerely Dr. Armando Navarro-Vázquez for inviting me to work for one month in his group at the University of Vigo. It turned out to be one of the most fruitful times during my PhD study. Many thanks for his guidance and help related with the computational studies, and the tremendous input in our joined publications.

I am very grateful to all our collaborators from whom we obtained all the intriguing compounds for our study: Prof. Carlos Kleber Z. Andrade and Prof. Rafael 
Oliveira Rocha for the new Michael addition reaction product; Dr. Emily L. Whitson and Prof. Chris M. Ireland for fibrosterol sulfate A; Prof. Ren-Xiang Tan and Dr. Hui-Ming Ge for vatiparol; Dr. Emily L. Whitson and Dr. Tawnya C. McKee for naphth 1 and naphth 2; Liang-Yan Liu and Prof. Ji-Kai Liu for LLG1; Dr. Matthias Köck for comp.540a.

I would like to sincerely thank Dr. Edward d'Auvergne for introducing me to computer programming using Python and numerous aid concerning the theoretical

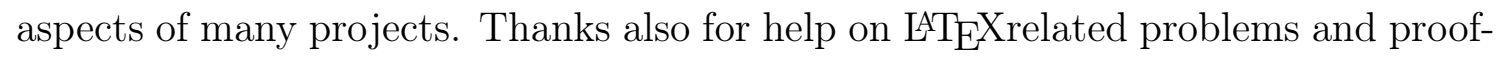
reading the abstract of this manuscript. I am very grateful to Manuel Schmidt for lots of scientific and technical support in the lab, for the great collaboration and many useful discussions. Furthermore, I also would like to thank Prof. Fernando Hallwass, Jens Pilger, Sibelle Cunha and Dr. Nilamoni Nath for the interesting discussions and inspirations. And thank you all for being such nice colleagues.

I am grateful to Dr. Per Rogne and Dr. Lars Kuhn for the guidance and help with the ECD measurements in their group, and Prof. Michael Reggelin and his coworkers for performing the ORD measurements for us.

I would like to thank Shengqi Xiang and Dr. Donghan Lee for the troubleshooting and practical help on the spectrometer. I would like to thank Dr. Venita Daebel for useful suggestions about thesis writing and graphical illustrations. I am grateful to Dr. Dirk Bockelmann for his continuous support with software installation and maintenance. I am also thankful to Dr. Zrinka Gattin for the installation of GROMOS and useful suggestions about the MD simulations. Many thanks to Dr. Dirk Matthes for teaching me how to perform MD simulations using GROMACS.

I am very grateful to all former and current colleagues in the NMRII department for the very pleasant and supportive working enviroment. I would like to thank all my friends for the nice time we shared together during and after the work. I really appreciate our precious friendships.

Most importantly I would like to deeply thank my husband, my parents, grandparents and all family members for the immeasurable support, trust and love throughout my life. 


\section{Contents}

Abstract $\quad$ V

$\begin{array}{lll}\text { Zusammenfassung } & \text { VII }\end{array}$

Related Publications

$\begin{array}{lll}\text { Acknowledgements } & \text { XI }\end{array}$

$\begin{array}{ll}\text { Contents } & \text { XIII }\end{array}$

List of Abbreviations $\quad$ XVII

1 Introduction 1

1.1 Chirality and stereochemistry . . . . . . . . . . . . . . 1

1.2 Stereochemical elucidation of natural products . . . . . . . . . . 3

1.3 Motivation and outline of this thesis . . . . . . . . . . . . 4

2 Theory 9

2.1 The phenomenon of nuclear magnetic resonance . . . . . . . . . . 9

2.2 Interactions in NMR spectroscopy . . . . . . . . . . . . . . . . 10

2.2.1 Chemical shielding . . . . . . . . . . . . . . 11

2.2 .2 Dipolar coupling . . . . . . . . . . . . . . . 13

$2.2 .3 \quad J$-coupling . . . . . . . . . . . . . . . . . . . . . . . . . . . 19

2.2.4 Quadrupolar coupling ................ . . 20

3 Materials and Methods $\quad 23$

3.1 Materials .......................... 23

3.1.1 Equipment . . . . . . . . . . . . . . . . 23

3.1 .2 Important chemicals . . . . . . . . . . . . . . . . 23

3.1.3 Software packages . . . . . . . . . . . . . . 23

3.2 Methods . . . . . . . . . . . . . . . . . . 26

3.2.1 NMR spectroscopy . . . . . . . . . . . . . . . 26

3.2.2 Circular dichroism $(\mathrm{CD}) \ldots \ldots \ldots \ldots . \ldots 27$ 
3.2.3 Optical rotatory dispersion (ORD) . . . . . . . . . . . . . 29

3.2.4 Molecular dynamics (MD) simulations and analysis . . . . . . 29

3.2.5 Molecular mechanics simulations . . . . . . . . . . . 30

3.2.6 Density functional theory (DFT) calculations . . . . . . . . 30

3.2.7 Analysis of residual dipolar couplings . . . . . . . . . . . . 31

3.2.8 Quantitative analysis of ROE and NOE data . . . . . . . . . 34

4 Stereochemical elucidation of molecules of class A 37

4.1 Introduction . . . . . . . . . . . . . . . . 37

4.2 Determination of the absolute configuration of LLG1 . . . . . . . 38

4.2 .1 Introduction . . . . . . . . . . . . . . . . 38

4.2.2 Elucidation of the relative configuration and conformation by NMR . . . . . . . . . . . . . . . . 39

4.2.3 Elucidation of the absolute configuration by chiroptics . . . . 44

4.2 .4 Conclusion . . . . . . . . . . . . . . . . . 48

4.3 Determination of the absolute configuration of vatiparol . . . . . . . . 49

4.3 .1 Introduction . . . . . . . . . . . . . . . . . 49

4.3.2 Elucidation of the relative configuration and conformation by NMR . . . . . . . . . . . . . . 5 50

4.3.3 Elucidation of the absolute configuration by chiroptics . . . . 58

4.3 .4 Conclusion . . . . . . . . . . . . . . 60

4.4 Determination of the absolute configuration of naphth 1 and naphth 262

4.4 .1 Introduction . . . . . . . . . . . . . . . 62

4.4.2 Elucidation of the absolute configuration by chiroptics . . . . 63

4.4 .3 Conclusion . . . . . . . . . . . . . 66

5 Stereochemical elucidation of molecules of class B1 69

5.1 Introduction . . . . . . . . . . . . . . . . . . . 69

5.2 Determination of the absolute configuration of a Michael addition product ...................... 70

5.2 .1 Introduction . . . . . . . . . . . . . 70

5.2.2 Elucidation of the relative configuration and conformation by NMR . . . . . . . . . . . . . . . . 72

5.2.3 Elucidation of the absolute configuration by ORD . . . . . . 83

5.2 .4 Conclusion . . . . . . . . . . . . . . . . 84

5.3 Configurational and conformational analysis of comp.540a . . . . . 86

5.3 .1 Introduction . . . . . . . . . . . . . . 86

5.3.2 Elucidation of the relative configuration of the macrolide ring 86 
5.3.3 Elucidation of the relative configuration between the macrolide ring and the sugar f . . . . . . . . . . . . . . . . . 98

5.3 .4 Conclusion . . . . . . . . . . . . . . . 100

6 Stereochemical elucidation of a molecule of class B2 103

6.1 Determination of the relative configuration of fibrosterol sulfate A . . 103

6.1.1 Introduction . . . . . . . . . . . . . . . 103

6.1.2 Elucidation of the relative configuration and conformation by multiple-tensor analysis . . . . . . . . . . . . 105

6.1.3 Elucidation of the relative configuration and conformation by single-tensor analysis . . . . . . . . . . . . . . . . 119

6.1.4 Comparison of the RDC-based single-tensor analysis and multipletensors analysis. . . . . . . . . . . . . . . . . . . 128

6.1.5 Validation of C20 and C20' configuration using RDC data . . 129

6.1.6 Conclusion . . . . . . . . . . . . . . . . 129

$\begin{array}{lll}7 & \text { Conclusions and outlook } & 131\end{array}$

7.1 Conclusions . . . . . . . . . . . . . . . . . . . . 131

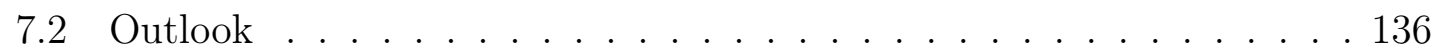

$\begin{array}{lr}\text { Bibliography } & 139\end{array}$

$\begin{array}{ll}\text { A Additional data } & 157\end{array}$

A.1 Additional data for LLG1 . . . . . . . . . . . . . . . . . . . . 157

A.2 Additional data for vatiparol . . . . . . . . . . . . . . . . 163

A.3 Additional data for naphth 1 and naphth 2 . . . . . . . . . . . 168

A.4 Additional data for the Michael addition product . . . . . . . . . . . 174

A.5 Additional data for comp.540a . . . . . . . . . . . . . . . . 180

A.6 Additional data for fibrosterol sulfate A . . . . . . . . . . . . . . 185

B Additional equations for thermodynamics 195

$\begin{array}{lr}\text { C Pulse program } & 199\end{array}$

$\begin{array}{ll}\text { Lebenslauf } & 203\end{array}$ 


\section{List of Abbreviations}

\begin{tabular}{|c|c|}
\hline AMPS & 2-Acrylamido-2-MethylPropane-1-Sulfonic acid \\
\hline APhES & 2-Acrylamido-2-PhenylEthane-1-Sulfonic acid \\
\hline $\mathrm{BACE}$ & $\beta$-secretase \\
\hline B3LYP & Becke, three-parameter, Lee-Yang-Parr \\
\hline CCL2 & Chemokine (C-C motif) Ligand 2 \\
\hline $\mathrm{CD}$ & Circular Dichroism \\
\hline $\mathrm{CFF}$ & Consistent Force Field \\
\hline CLIP-HSQC & CLean InPhase Heteronuclear Single-Quantum Coherence \\
\hline COSY & COrrelation SpectroscopY \\
\hline CS & Chemical Shift \\
\hline CVFF & Consistent Valence Force Field \\
\hline DDD & Distance-bounds-Driven Dynamics \\
\hline DFT & Density Functional Theory \\
\hline DG & Distance Geometry \\
\hline DMSO & DiMethylSulfOxide \\
\hline DNP & Dictionary of Natural Products \\
\hline ECD & Electronic Circular Dichroism \\
\hline E.COSY & Exclusive COrrelation SpectroscopY \\
\hline EFG & Electric-Field Gradient \\
\hline $\mathrm{EM}$ & ElectroMagnetic \\
\hline fc & Floating Chirality \\
\hline fc-rDG & Floating Chirality Restrained Distance Geometry \\
\hline
\end{tabular}


HECADE HEteronuclear Couplings from ASSCI-Domain experiments with E.COSY-type crosspeaks

HMBC Heteronuclear Multiple Bond Correlation

HRESIMS High-Resolution Electron Spray Ionization Mass Spectroscopy

HSQC Heteronuclear Single-Quantum Coherence

HSQMBC Heteronuclear Single Quantum Multiple Bond Correlation

IEFPCM

Integral Equation Formalism variant Polarizable Continuum Model

MCP-1 Monocyte Chemo-attractant Protein 1

MD

Molecular Dynamics

NCI

National Cancer Institute

NMR

Nuclear Magnetic Resonance

NOE

Nuclear Overhauser Effect

NOESY

Nuclear Overhauser Effect SpectroscopY

ORD

Optical Rotatory Dispersion

PAF

Principal Axis Frame

PAN

Poly(AcetoNitrile)

PAS

Principal Axis System

P.E.COSY Primitive Exclusive COrrelation SpectroscopY

PEEK PolyEther Ether Ketone

pdb Protein Data Bank

PKC Protein Kinase C

PPARs Peroxisome Proliferator-Activated Receptors

ppm Parts Per Million (relative unit of nuclear chemical shift)

RCSA Residual Chemical Shift Anisotropy

RDC Residual Dipolar Coupling

rDG Restrained Distance Geometry

RF Radio Frequency

RMSD Root-Mean-Square Deviation 
r-MD Restrained Molecular Dynamics

ROA Raman Optical Activity

ROE

Rotating frame Overhauser Effect

ROESY

Rotating frame Overhauser Effect SpectroscopY

SVD

Singular Value Decomposition

TD-DFT

Time-Dependent Density Functional Theory

TOCSY

TOtal Correlation SpectroscopY

TRAIL

Tumor necrosis factor- $\alpha$-Related Apotosis-Inducing Ligand

TS

Transition State

VCD

Vibrational Circular Dichroism 


\section{Chapter 1}

\section{Introduction}

\subsection{Chirality and stereochemistry}

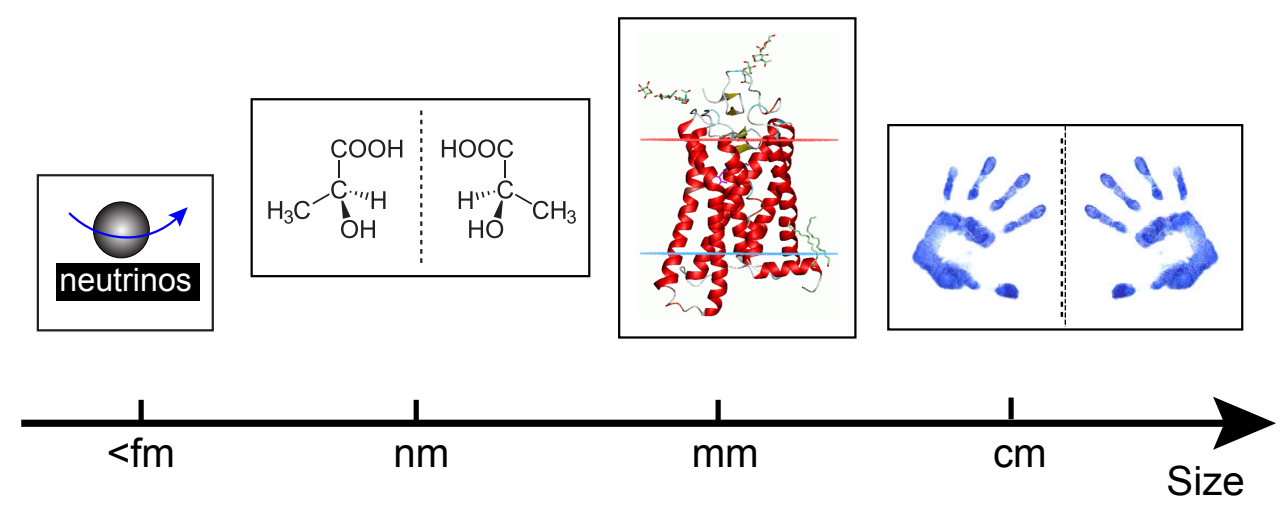

Figure 1.1: Chiral objects exist on all length scales.

Chirality is a property of objects that are not identical to their mirror images, i.e. image and mirror image are non-superimposable. As illustrated in Figure 1.1, the phenomenon of chirality can be discovered in almost all domains and dimensions in science and in our life [Janoschek, 1991], for instance elementary particles in physics, most organic molecules, $\alpha$-helices in proteins, and one of the most common examples which are our hands.

Stereochemistry, in which one of the most important branches is the study of the chirality in chemistry, deals with the three-dimensional arrangement of atoms within molecules. The phenomenon of stereochemistry was firstly described by Louis Pasteur during the period from 1848 to 1853 as he discovered that circularly polarized light could be rotated differently by salts of tartaric acid that were collected from different sources. Today, studying stereochemistry has become very essential in un- 
derstanding many problems in organic, inorganic, physical, analytical, biochemical and supramolecular chemistries [Eliel et al., 2005].

Molecules that have the same constitution and conformation but only differ in their absolute configuration are called enantiomers, which are mirror images of each other but not identical to each other, and are chiral. Enantiomers have the same chemical and physical properties and are thus very difficult to be distinguished. The first assignment of absolute configuration of an organic molecule was accomplished by Johannes Martin Bijvoet in 1951 with anomalous X-ray diffraction [Bijvoet et al., 1951]. Since this so-called "Bijvoet method" is based on the anomalous scattering which only occurs when the wavelength of the X-rays is near the absorption edge of the heavy atoms, a heavy atom with atomic mass larger than sulfur in the molecule is required. For many other molecules, the absolute configuration was related to other compounds often by chemical synthesis, which also means that the absolute configuration of all these chemically related molecules was solved. Nowadays, the methods applied for determining absolute stereochemistry have been significantly expanded and can be summarized in the following categories [Allenmark and Gawronski, 2008]: (i) Synthetic methods [Nicolaou and Snyder, 2005]: total synthesis, degradation reaction, converting the studied molecule into a compound with known configuration, and X-ray analysis of the synthetically converted Mosher ester; (ii) anomalous X-ray diffraction [Bijvoet et al., 1951]; (iii) chiroptical spectroscopy [Petrovic et al., 2010]: optical rotary dispersion (ORD), electronic circular dichroism (ECD), vibrational circular dichroism (VCD), and raman optical activity (ROA); (iv) empirical nuclear magnetic resonance (NMR) based methods [Seco et al., 2004]: e.g. Mosher ester analysis; (iv) chromatographic methods. However, since each method has its own limitations, for example, monocrystals of good quality with at least one heavy atom inside the molecule are required for anomalous X-ray crystallography, and Mosher ester analysis is restricted to molecules that have a functional group that can be derivatized (alcohols, amines, etc.), further method development for studying the absolute configuration is still demanded.

Molecules that have a different relative stereochemistry are called diastereomers. They have different physical and chemical properties and are often given different names, e.g. menthol and neomenthol. For the analysis of the relative configuration of organic molecules, one of the most frequently applied techniques is NMR spectroscopy, which relies in most cases on a combination of proton-proton distance estimation from nuclear Overhauser effect (NOE) intensities and determination of dihedral angles through Karplus analysis of scalar ${ }^{2,3} \mathrm{~J}$-couplings. In the last ten 
to fifteen years, ab initio computations [Bifulco et al., 2007, Di Micco et al., 2010], mostly DFT based, of chemical shifts and scalar couplings have proven to be useful as well for the establishment of the constitution and configuration of medium-sized organic molecules. However, for many molecules, ambiguities about the configuration still remain, especially when conformationally heterogeneous molecules with multiple stereocenters are analyzed.

\subsection{Stereochemical elucidation of natural products}

Natural products are chemical compounds that are produced by living organisms. They are also called secondary metabolites, because they are not essential for the life of their producers, however, are highly interesting because of their biological effect on other organisms [Hanson, 2003]. Furthermore, due to their variety and structural diversity, natural products are until now still a big reservoir for the discovery of new chemical entities with pharmaceutical, agrochemical and nutraceutical activities. Studies by Newman and Cragg show that almost 50\% of the new drugs introduced from 1981 to 2010 have a natural product origin [Newman and Cragg, 2007, Newman and Cragg, 2012].

Chirality is a key property of natural products. A statistical study from 2006 shows that about $80 \%$ of 161278 natural products from the Dictionary of Natural Products (DNP, dnp.chemnetbase.com) are chiral. The average number of chiral centers of the natural products in the DNP is 5.19 , whereas the number is 0.39 for a representative set of 24725 synthetic compounds, that are randomly sampled from a database containing 4.8 million screening samples [Mannhold et al., 2006]. This result indicates that natural products are "more chiral" than synthetic compounds and one possible explanation for this lies in the chirality of the enzymatic environment in the biosynthesis. Furthermore, various studies also show the importance and the complexity of the stereochemistry for the expression of bioactivity of natural products. Kenji Mori, a well-known researcher in the field of stereochemistry of natural products, has nicely summarized the stereochemistry-bioactivity relationships in one of his latest reviews in 2011 [Mori, 2011]. He pointed out, that the generally accepted view that only one certain enantiomer is responsible for the activity is not a fixed rule in nature. Also other various examples show different relationships between configuration and bioactivity, e.g. both enantiomers are necessary for the bioactivity, or even in the same genus different species use different enantiomers.

In most cases, structure elucidation is the first step for studying a natural prod- 
uct. Many important aspects, e.g. structure-activity relationship [Mori, 2011] or protein-ligand interactions, can only be studied after the structure including configuration of a compound has been determined. Conventionally, the configuration of natural products is studied by NMR, X-ray, synthetic approaches or chiroptical methods, as already described in the previous section. However, compared to synthetic organic molecules, stereochemical elucidation of natural products is significantly more challenging due to the following difficulties: (i) limited amount of sample: natural products isolated from embedded sources or species that are difficult to collect often only give trace amounts of sample; (ii) determination of absolute configuration: different from synthetic compounds where the absolute configuration can be derived from the stereo-information of the chiral educts, in natural products initially nothing is known about the configuration.

\subsection{Motivation and outline of this thesis}

Structure determination, in particular configuration of complex natural products is in many cases a formidable task. Often, ambiguities about the configuration still remain, when the conventional NMR-based methods such as NOE and J-coupling analysis are applied. However, this situation has been changed in last 10 years after the development of new weakly aligning media compatible with organic solvents, such as liquid crystal solutions [Marx and Thiele, 2009, Arnold et al., 2010, Thiele et al., 2011] or mechanically stretched cross-linked swollen polymers [Freudenberger et al., 2004, Freudenberger et al., 2005, Haberz et al., 2005, Luy et al., 2005, Kummerlöwe et al., 2007, Gil et al., 2008, Kummerlöwe et al., 2009]. These media impose a certain degree of orientational ordering on the studied solute molecules, and parameters hidden in isotropic solutions such as residual dipolar couplings (RDCs) or residual chemical shift anisotropies (RCSAs) become observable. Due to the fact that changes of sample conditions have only an insignificant influence on the scalar couplings, residual dipolar couplings can be directly measured by subtracting the couplings measured for the aligned sample from those in solution. In contrast, it is well known that chemical shifts are very sensitive to the sample conditions. Therefore, when residual chemical shift anisotropies are extracted, an additional contribution to the chemical shift changes due to sample variation from isotropic to anisotropic conditions needs to be considered [Hallwass et al., 2011, Kummerlöwe et al., 2011]. From the structural point of view, residual dipolar couplings provide valuable information about the relative orientation of internuclear vectors with respect to the molecular frame. The usage of dipolar couplings measured for different 
regions makes it possible to correlate distant parts within a molecule and thus provides long-range structural information which complements NOEs, J-couplings and chemical shifts that are short-range in nature. [Kummerlöwe and Luy, 2009, Sun et al., 2011b].

The stereochemical elucidation of small molecules based on the RDC method was firstly applied to conformationally rigid molecules, such as the prochiral protons assignment of strychnine [Thiele and Berger, 2003] and the determination of relative configuration of a five-membered ring [Yan et al., 2004]. The RDC analysis for these molecules uses the theoretical interpretation of the dipolar couplings in terms of the alignment tensor [Kramer et al., 2004]. It is a three by three matrix that contains five independent elements, which can be determined when five or more experimental $\mathrm{RDC}$ values are available. In case the molecule has a rigid conformation, the correctness of the structure can be evaluated directly by comparing the back-calculated RDCs from the determined alignment tensor with the experimentally measured ones.

For flexible systems, the experimentally measured RDC values are dynamically averaged because of their orientational dependence and therefore difficult to be interpreted in the analysis. In 2007, the relative stereochemistry of sagittamide A, that contains 6 unknown stereocenters in a long flexible acyclic chain, has been successfully studied using RDC-based analysis. [Schuetz et al., 2007] In this work, a "single-tensor" approach for an ensemble of molecules has been proposed for solving the problem of conformational heterogeneity. In the last five years, this approach has been applied to various systems by different groups [Schuetz et al., 2007, Sánchez-Pedregal et al., 2009, Thiele et al., 2009, Gayathri et al., 2010, Sun et al., 2011a, Kramer and Kleinpeter, 2011, Schmidt et al., 2012]. Nevertheless, if the shapes of the various conformations are very different, the "single-tensor" method may no longer be considered valid, and an individual alignment tensor needs to be calculated for each conformation. This approach, however, has been employed before this thesis only on simple model systems, e.g. $\alpha$-methylene- $\gamma$-butyrolactone [Thiele et al., 2009]. During this PhD work, on one hand, we extensively applied the "singletensor" approach to four complex systems and proposed different strategies to evaluate the NMR data for different classes of molecules, which will be presented in Chapters 4, 5 and 6. On the other hand, we have employed the "multiple-tensor" RDC analysis, combined with NOE and scalar coupling restraints, for studying a bissteroid, in which the two steroid bodies are connected by a highly flexible linker [Sun et al., 2011b]. Explicit solvent molecular dynamics (MD) simulations were employed in this study to sample the conformational space and calculate the main conforma- 
tions together with their population. This is the first time that the stereochemistry of a complex natural product with very different global conformations has been elucidated. This part of the work will be described in Chapter 6 .

Over the past two decades, chiroptical techniques such as optical rotatory dispersion, electronic circular dichroism and vibrational circular dichroism has emerged as a powerful tool to determine the absolute configuration of chiral molecules. However, as this requires all conformations to be known, these techniques are still mostly applied to relatively rigid compounds. Due to this limitation, another focus of this $\mathrm{PhD}$ thesis is to propose a new methodology to overcome the flexibility issue, which has been achieved by combining RDC-enhanced NMR spectroscopy for the determination of the precise conformation and relative configuration with chiroptical spectroscopy and computational methods such as DFT calculations to further elucidate the absolute configuration of natural products. A similar method has been applied firstly for the relatively rigid molecule dibromopalau'amine [Reinscheid et al., 2010]. The confirmation of the determined absolute configuration came one year later by organic synthesis [Seiple et al., 2011]. This combined approach has been continuously applied in this $\mathrm{PhD}$ thesis and further developed on several challenging flexible systems [Sun et al., 2011a, Ge et al., 2012]. In this contribution, a new fundamental methodology overcoming the flexibility issue to resolve the absolute configuration will be presented. Rather than sampling the entirety of the conformational space, ensembles derived by NMR spectroscopy, which reproduced the experimental NMR spectroscopic data were used to predict chiroptical properties of the molecule in solution. The obtained results will be presented in Chapters 4 and 5 .

Before this thesis all reported successful applications of the methodology using anisotropic NMR parameters have been performed with conventional $5 \mathrm{~mm}$ NMR tubes, which require a relatively large amount $(>5 \mu \mathrm{mol})$ of sample to compensate for the low sensitivity and substantial background signal from the alignment medium used to achieve anisotropic conditions. This limitation hampers measurements for many natural products of poor availability $(<3 \mu \mathrm{mol})$ and precludes the determination of their configuration by NMR [Sun et al., 2010]. The sensitivity problem of NMR spectroscopy has been significantly reduced since the commercial $1.7 \mathrm{~mm}$ microprobe became available in 2007. The usage of the microprobe has enabled structure elucidation of natural products with very restricted amounts in several studies [Molinski, 2009, Dalisay and Molinski, 2010, Molinski, 2010a]. However, this advantage has not yet been utilized for RDC measurements, probably due to the difficulties in preparing the alignment media for $1.7 \mathrm{~mm}$ tubes given their very small 
diameter. In this thesis, the first-time preparation of a slim PH-gel for $1.7 \mathrm{~mm}$ NMR tubes in a reproducible and efficient way [Ge et al., 2012] will be described. With this preparation, the required amount could be reduced to 1-3 $\mu$ mol. Furthermore the NMR spectra recorded with these slim NMR tubes are comparable or have even better quality than those from conventional $5 \mathrm{~mm}$ tubes. The results will be discussed in Chapter 4.
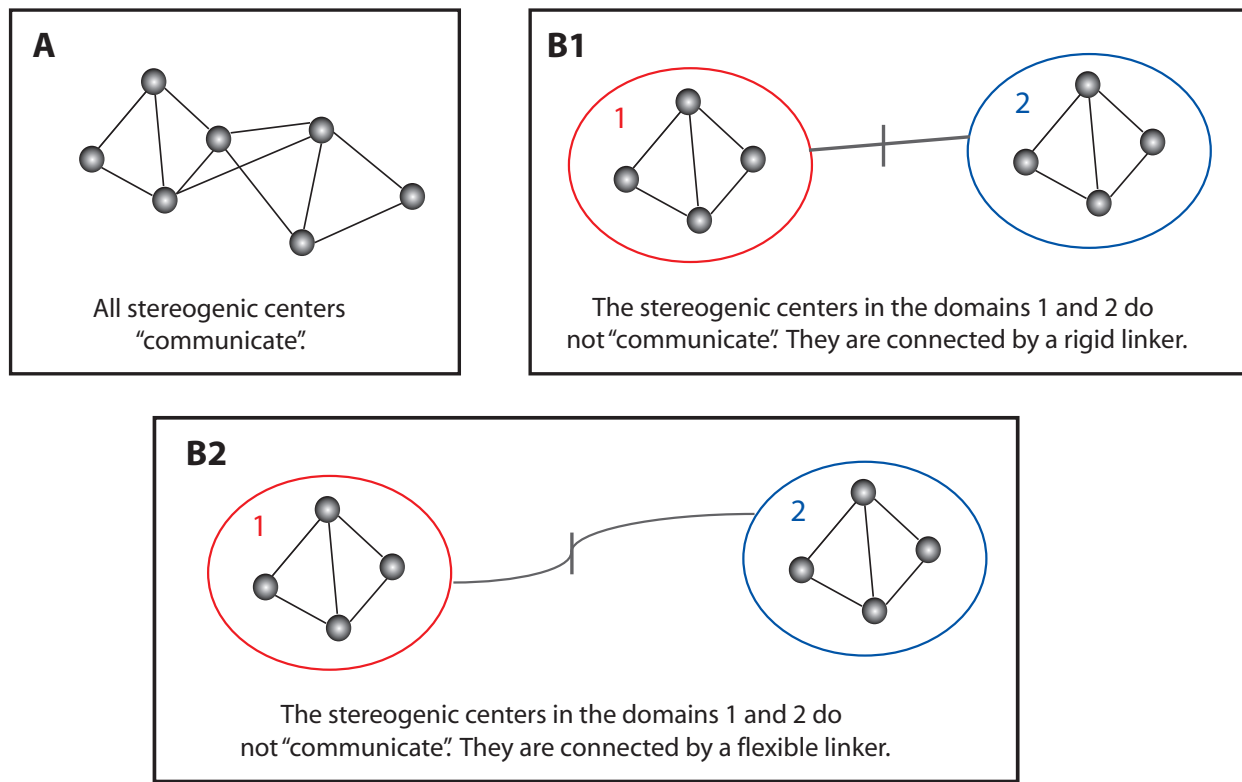

Figure 1.2: Categorization of chiral molecules based on conformational flexibilities.

During this $\mathrm{PhD}$ work in total the relative/absolute configuration of six natural products and one synthetic molecule has been determined. Each molecule posed different difficulties and challenges for the elucidation of their configuration. Since conformational flexibility was the central problem, these seven molecules have been divided into three different categories A, B1 and B2, based on the flexibility and connectivity of all stereogenic centers within one molecule. The classification as illustrated in Figure 1.2 follows the one in the proposal of the DFG research unit 934. In this work, Chapters 4, 5 and 6 present the results of the stereochemical elucidation of molecule classes A, B1 and B2, respectively, forming the core of this thesis. With this way a general methodology for the stereochemical determination of challenging natural products is proposed: it uses RDC-based NMR analysis combined with computational calculations and chiroptical spectroscopy. 


\section{Chapter 2}

\section{Theory}

\subsection{The phenomenon of nuclear magnetic resonance}

Spectroscopy is the study of the interaction between matter and electromagnetic (EM) radiation and is a fundamental method for investigation of physical properties of matter at different length scales, such as materials, crystals, molecules, atoms, nuclei, electrons, etc. Figure 2.1 shows different frequency regions of EM waves that are employed for the radiation in the most frequently applied spectroscopy techniques.

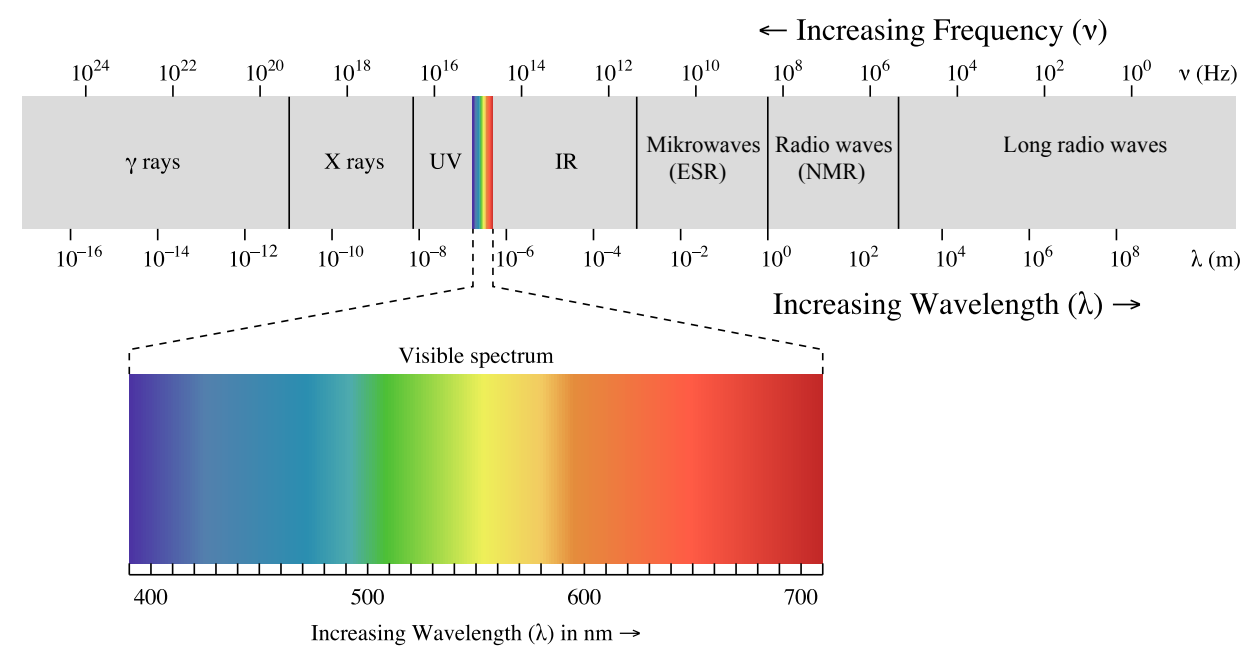

Figure 2.1: The electromagnetic spectrum. This figure was taken from wikipedia (en.wikipedia.org/wiki/File:EM_spectrum.svg) and modified accordingly.

Nuclear magnetic resonance is a spectroscopy technique in the radio frequency $(\mathrm{RF})$ range, which relies on the effect that $\mathrm{RF}$ waves $(\mathrm{MHz})$ are able to perturb 
the spin states of nuclei, when an external magnetic field (1-23.5 T) is present. To some extent, NMR spectroscopy follows the same principles as optical spectroscopy, in particular Fermi's golden rule and the selection rules apply. Historically, one group [Purcell et al., 1946] at Harvard has indeed invented NMR spectroscopy on bulk material by the inspiration from optical atomic spectroscopy. In their work they have described the frequency $\omega_{0}$ of the transition between two quantized Zeeman levels as the basic resonance condition:

$$
\omega_{0}=\frac{\Delta E}{\hbar}=-\gamma B_{0}
$$

with the gyromagnetic ratio $\gamma$ and external static magnetic field strength $B_{0}$. Nevertheless, in the same year of 1946, another group [Bloch et al., 1946] at Stanford University independently invented NMR on bulk material by considering magnetic moments as being reoriented with respect to the external magnetic field and rotating at the Larmor frequency $\omega_{L}$. In contrast to Purcell's quantum description, this approach has been called later the classical or macroscopic description. Although these two approaches are apparently very different, they describe in fact the same phenomenon. The quantum transition frequency $\omega_{0}$ is equal to the Larmor frequency $\omega_{L}$.

\subsection{Interactions in NMR spectroscopy}

In quantum mechanics, the Hamiltonian operator corresponds to the total energy in classical mechanics, and determines the time evolution of a system and describes the corresponding interactions in a quantified manner. In NMR spectroscopy, there are two components of spin Hamiltonian that need to be considered: one is based on the interaction between the spins and the external magnetic fields $\left(\mathscr{H}_{e x}\right)$, and the other is based on the internal interaction among the spins $\left(\mathscr{H}_{\text {int }}\right)$ :

$$
\mathscr{H}=\mathscr{H}_{\text {ex }}+\mathscr{H}_{\text {int }} .
$$

The external Hamiltonian consists of $\mathscr{H}_{\text {Zeeman }}$, which describes the interaction between the spins and the static magnetic field $B_{0}$ that is essential for observations of NMR signals, and $\mathscr{H}_{R F}$, which is present when the oscillating radio frequency field is switched on. The Hamiltonians for these two interactions are written as [Cavanagh 
et al., 2006]:

$$
\begin{aligned}
\mathscr{H}_{\text {Zeeman }} & =-\gamma B_{0} I_{z} \\
\mathscr{H}_{R F} & =\omega_{1}\left[I_{x} \cos \left(\omega_{r f} t+\phi\right)+I_{y} \sin \left(\omega_{r f} t+\phi\right)\right] \\
& =-\gamma B_{1}\left[I_{x} \cos \left(\omega_{r f} t+\phi\right)+I_{y} \sin \left(\omega_{r f} t+\phi\right)\right]
\end{aligned}
$$

where $I_{x}, I_{y}$ and $I_{z}$ are the spin angular momentum operators along the axes $x, y$ and $z$, respectively, and $B_{1}$ is the field strength of the transverse RF field.

The internal interaction of spins comprise the chemical shielding $\left(\mathscr{H}_{C S}\right)$, the $J$-coupling $\left(\mathscr{H}_{J}\right)$, the dipolar coupling $\left(\mathscr{H}_{D}\right)$, and the quadrupolar coupling $\left(\mathscr{H}_{Q}\right)$ :

$$
\mathscr{H}_{\text {int }}=\mathscr{H}_{C S}+\mathscr{H}_{J}+\mathscr{H}_{D}+\mathscr{H}_{Q}
$$

where the quadrupolar coupling interactions only exist for spin $I>\frac{1}{2}$.

\subsubsection{Chemical shielding}

The chemical shift is the most important parameter in NMR spectroscopy for characterizing atoms in a molecule. The chemical shift difference of individual atoms with different electronic environment stems from the modification of the Zeeman interactions by the chemical shielding, and thus slightly changes the resonance frequency. Due to the fact that the electronic charge distribution is anisotropic in a molecule, the Hamiltonian of the chemical shielding can be expressed in terms of a second rank tensor $\boldsymbol{\sigma}$ as:

$$
\mathscr{H}_{C S}=\gamma \mathbf{I} \boldsymbol{\sigma} \mathbf{B}_{\mathbf{0}}
$$

where $\mathbf{I}$ is an operator that represents the nuclear spin angular momentum. In general, the chemical shielding tensor can be represented by a $(3 \times 3)$ matrix [Cavanagh et al., 2006], and is able to be decomposed into independent symmetric and antisymmetric parts, of which only the symmetric one contributes to the chemical shift. In the principal axis frame (PAF), only the diagonal elements have non-zero values and these three elements $\left(\sigma_{x x}, \sigma_{y y}, \sigma_{z z}\right)$ are the important parameters for defining the isotropic shielding constant $\sigma_{\mathrm{o}}$, the chemical shift anisotropy $\Delta \sigma$ and the chemical shift asymmetry $\eta$ [Grant, 2007]:

$$
\begin{gathered}
\sigma_{\mathrm{o}}=\frac{\sigma_{x x}+\sigma_{y y}+\sigma_{z z}}{3}, \\
\Delta \sigma=\sigma_{z z}-\frac{\left(\sigma_{x x}+\sigma_{y y}\right)}{2},
\end{gathered}
$$




$$
\eta=\frac{\sigma_{x x}-\sigma_{y y}}{\sigma_{x x}-\sigma_{\mathrm{o}}}
$$

Due to the fact that the trace of a tensor does not change when the tensor is rotated, the isotropic shielding constant always remains unchanged. In contrast, the anisotropic part of the chemical shift depends on the orientation of the molecules in the magnetic field, which can be expressed in terms of the polar angles $(\theta, \phi)$ of the $\mathbf{B}_{0}$ field in the principal axis system (PAS) [Schmidt-Rohr and W, 1996]. The chemical shift anisotropy contains one axial part $\left(3 \cos ^{2} \theta-1\right)$ and a rhombic part $\left(\sin ^{2} \theta \cos 2 \phi\right)$. In solid-state NMR without magic angle spinning, since many different orientations are present in a powder, not a single line but a typical powder pattern can be observed in the spectrum. On the other hand this pattern reflects the property of the shielding tensor, which is characteristic of the symmetry in the molecule. In a weakly aligned sample, certain orientations are slightly preferential than the others, hence a small portion of the chemical shift anisotropy from this orientation contributes additionally to the chemical shift. The relationship between this so-called residual chemical shift anisotropy and the orientational properties of molecules can be expressed as [Losonczi et al., 1999]:

$$
\delta_{a n}=\frac{2}{3} \sum_{k=\{x, y, z\}}\left\langle\frac{3 \cos ^{2} \Theta_{k}-1}{2}\right\rangle \delta_{k k},
$$

where $\delta_{a n}$ is the residual chemical shift anisotropy, $\delta_{k k}$ are the elements of the diagonal chemical shift tensor in the principal axis frame (PAF), $\Theta_{k}$ are the angles between the principal axis frame of the chemical shift tensor and the external magnetic field. The angle brackets in the equation denote a time average due to motion. In the arbitrary molecular frame, the residual chemical shift anisotropy can be written as:

$$
\delta_{a n}=\frac{2}{3} \sum_{k, l=\{x, y, z\}} A_{k l} \delta_{k l},
$$

in which $\mathbf{A}$ is a symmetric and traceless tensor. This order matrix is identical to the one that is used in the residual dipolar coupling analysis to define the orientation of the molecule in the partially ordered medium. The property of this tensor will be described extensively in the section on dipolar coupling (Section 2.2.2). In practice, to determine the five independent components of the alignment tensor from the residual chemical shift anisotropies, one can solve the linear equation that has the form of [Losonczi et al., 1999]: 


$$
\left(\begin{array}{ccccc}
-\delta_{11}^{1}+\delta_{22}^{1} & -\delta_{11}^{1}+\delta_{33}^{1} & -\delta_{12}^{1}+\delta_{21}^{1} & -\delta_{13}^{1}+\delta_{31}^{1} & -\delta_{23}^{1}+\delta_{32}^{1} \\
-\delta_{11}^{2}+\delta_{22}^{2} & -\delta_{11}^{2}+\delta_{33}^{2} & -\delta_{12}^{2}+\delta_{21}^{2} & -\delta_{13}^{2}+\delta_{31}^{2} & -\delta_{23}^{2}+\delta_{32}^{2} \\
-\delta_{11}^{3}+\delta_{22}^{3} & -\delta_{11}^{3}+\delta_{33}^{3} & -\delta_{12}^{3}+\delta_{21}^{3} & -\delta_{13}^{3}+\delta_{31}^{3} & -\delta_{23}^{3}+\delta_{32}^{3} \\
-\delta_{11}^{4}+\delta_{22}^{4} & -\delta_{11}^{4}+\delta_{33}^{4} & -\delta_{12}^{4}+\delta_{21}^{4} & -\delta_{13}^{4}+\delta_{31}^{4} & -\delta_{23}^{4}+\delta_{32}^{4} \\
\cdot & \cdot & \cdot & \cdot & \cdot \\
\cdot & \cdot & \cdot & \cdot & \cdot \\
\cdot & \cdot & \cdot & \cdot & \cdot \\
-\delta_{11}^{n}+\delta_{22}^{n} & -\delta_{11}^{n}+\delta_{33}^{n} & -\delta_{12}^{n}+\delta_{21}^{n} & -\delta_{13}^{n}+\delta_{31}^{n} & -\delta_{23}^{n}+\delta_{32}^{n}
\end{array}\right)\left(\begin{array}{c}
A_{y y} \\
A_{z z} \\
A_{x y} \\
A_{x z} \\
A_{y z}
\end{array}\right)=\left(\begin{array}{c}
\delta_{a n}^{1} \\
\delta_{a n}^{2} \\
\delta_{a n}^{3} \\
\delta_{a n}^{4} \\
\cdot \\
\cdot \\
\cdot \\
\delta_{a n}^{n}
\end{array}\right)
$$

\subsubsection{Dipolar coupling}

The dipolar coupling results from the direct interaction between two nuclear magnetic moments through space. For nuclei with $I=\frac{1}{2}$, it is the most dominant interaction in solid state NMR, with a magnitude of $\mathrm{kHz}$. In the weakly aligned sample, the size of the dipolar couplings can be scaled down to the order of the $J$ couplings or below, which allows to obtain high-resolution NMR spectra with sharp lines. More importantly, this scaled dipolar coupling, the so-called residual dipolar coupling, provides valuable information about the relative orientation of internuclear vectors with respect to the molecular frame, and therefore has been proven in the last fifteen years to be a very important parameter for studying structure and dynamics of biological and organic molecules [Tjandra and Bax, 1997, Blackledge, 2005, Thiele, 2008]. In this thesis, residual dipolar couplings are a key source of information for the determination of configuration and conformation of organic molecules. In the following the theoretical aspects of the dipolar coupling and residual dipolar coupling will be explicitly discussed.

In general, the Hamiltonian of the dipolar coupling has the form of [Ernst et al., 2004]:

$$
\mathscr{H}_{D}=\mathbf{I}_{\mathbf{i}} \mathbf{D}_{\mathbf{i j}} \mathbf{I}_{\mathbf{j}}=d_{i j}\left(\mathbf{I}_{\mathbf{i}} \mathbf{I}_{\mathbf{j}}-3 \frac{1}{r_{i j}^{2}}\left(\mathbf{I}_{\mathbf{i}} \mathbf{r}_{\mathbf{i j}}\right)\left(\mathbf{I}_{\mathbf{j}} \mathbf{r}_{\mathbf{i j}}\right)\right),
$$

with $d_{i j}=\mu_{0} \gamma_{i} \gamma_{j} \hbar /\left(4 \pi r_{i j}^{3}\right)$, in which $\gamma_{i}$ and $\gamma_{j}$ are the gyromagnetic ratios of each interacting nucleus; $\mathbf{r}_{\mathbf{i}}$ is the internuclear vector; $\mu_{0}$ is the permeability of vacuum. In polar coordinates, the dipolar Hamiltonian can be expanded in terms of irreducible 
tensor operators as follows:

$$
\mathscr{H}_{D}=\sum_{q=-2}^{2} F_{i j}^{(q)} A_{i j}^{(q)}
$$

where $A_{i j}^{(q)}$ contains the spin operators:

$$
\begin{aligned}
A_{i j}^{(0)} & =d_{i j}\left\{I_{i z} I_{j z}-\frac{1}{4}\left(I_{i}^{+} I_{j}^{-}+I_{i}^{-} I_{j}^{+}\right)\right\}, \\
A_{i j}^{(1)} & =-\frac{3}{2} d_{i j}\left(I_{i z} I_{j}^{+}+I_{i}^{+} I_{j z}\right), \\
A_{i j}^{(-1)} & =-\frac{3}{2} d_{i j}\left(I_{i z} I_{j}^{-}+I_{i}^{-} I_{j z}\right), \\
A_{i j}^{(2)} & =-\frac{3}{4} d_{i j} I_{i}^{+} I_{j}^{+}, \\
A_{i j}^{(-2)} & =-\frac{3}{4} d_{i j} I_{i}^{-} I_{j}^{-},
\end{aligned}
$$

and $F_{i j}^{(q)}$ describes the orientational properties of the internuclear vector:

$$
\begin{aligned}
F_{i j}^{(0)} & =1-3 \cos ^{2} \theta_{i j}, \\
F_{i j}^{(1)} & =\sin \theta_{i j} \cos \theta_{i j} \exp \left(-i \phi_{i j}\right), \\
F_{i j}^{(-1)} & =\sin \theta_{i j} \cos \theta_{i j} \exp \left(i \phi_{i j}\right), \\
F_{i j}^{(2)} & =\sin ^{2} \theta_{i j} \exp \left(-2 i \phi_{i j}\right), \\
F_{i j}^{(-2)} & =\sin ^{2} \theta_{i j} \exp \left(2 i \phi_{i j}\right),
\end{aligned}
$$

in which $\theta_{i j}$ is the angle between the external magnetic field $\mathbf{B}_{\mathbf{0}}$ and the internuclear vector $\mathbf{r}_{\mathbf{i j}}$, and $\phi_{i j}$ is the azimuthal angle with respect to the $\mathrm{x}$-axis. Using the concept of the "dipolar alphabet" that depends on the functions $A_{i j}^{(q)}$ and $F_{i j}^{(q)}$ :

$$
\begin{aligned}
A+B & =\frac{1}{d_{i j}} F_{i j}^{(0)} A_{i j}^{(0)}, \\
C & =\frac{1}{d_{i j}} F_{i j}^{(1)} A_{i j}^{(1)}, \\
D & =\frac{1}{d_{i j}} F_{i j}^{(-1)} A_{i j}^{(-1)}, \\
E & =\frac{1}{d_{i j}} F_{i j}^{(2)} A_{i j}^{(2)}, \\
F & =\frac{1}{d_{i j}} F_{i j}^{(-2)} A_{i j}^{(-2)},
\end{aligned}
$$


the Hamiltonian of the dipolar coupling can be reformulated as:

$$
\mathscr{H}_{D}=d_{i j}(A+B+C+D+E+F) .
$$

Under the high-field approximation, in which the Zeeman interaction is much larger than the dipolar interaction, only terms $\mathrm{A}$ and $\mathrm{B}$ that commute with the Zeeman interaction $\left(I_{z}\right)$ retain, whereas the non-secular terms C, D, E and F, affected by the time evolution under $I_{z}$, average to zero. After the truncation of these non-secular terms, the dipolar Hamiltonian in Equation 2.13 can be simplified to:

$$
\mathscr{H}_{D}=\frac{1}{2} d_{i j}\left(1-3 \cos ^{2} \theta_{i j}\right)\left(2 I_{i z} I_{j z}-\frac{1}{2}\left(I_{i}^{+} I_{j}^{-}+I_{i}^{-} I_{j}^{+}\right)\right) .
$$

Due to the following relationship between the Cartesian angular momentum operators and the raising and lowering operators:

$$
\begin{aligned}
I_{i}^{+} I_{j}^{-} & =\frac{1}{2}\left(2 I_{i x} I_{j x}+2 I_{i y} I_{j y}-i 2 I_{i x} I_{j y}+i 2 I_{i y} I_{j x}\right), \\
I_{i}^{-} I_{j}^{+} & =\frac{1}{2}\left(2 I_{i x} I_{j x}+2 I_{i y} I_{j y}-i 2 I_{j x} I_{i y}+i 2 I_{j y} I_{i x}\right),
\end{aligned}
$$

the dipolar Hamiltonian can be further simplified to:

$$
\mathscr{H}_{D}=\frac{1}{2} d_{i j}\left(1-3 \cos ^{2} \theta_{i j}\right)\left(2 I_{i z} I_{j z}-I_{i x} I_{j x}-I_{i y} I_{j y}\right)
$$

For heteronuclear spin systems and homonuclear spin systems under the weak coupling regime, the transverse spin operators can be neglected, which leads to a further simplification to:

$$
\begin{aligned}
\mathscr{H}_{D} & =d_{i j}\left(1-3 \cos ^{2} \theta_{i j}\right) I_{i z} I_{j z}, \\
& =2 \pi D_{i j} I_{i z} I_{j z},
\end{aligned}
$$

where the dipolar coupling constant $D_{i j}$ is defined as:

$$
D_{i j}=-\frac{\mu_{0} \gamma_{i} \gamma_{j} \hbar}{4 \pi^{2} r_{i j}^{3}} \frac{\left(3 \cos ^{2} \theta_{i j}-1\right)}{2} .
$$

The dipolar coupling constant reaches its maximum value $D_{\max }$, when the internuclear vector is aligned with the magnetic field $\left(\theta_{i j}=0^{\circ}\right.$ or $\left.180^{\circ}\right)$. Dipolar couplings are zero in solution due to complete averaging of all orientations, while residual dipolar couplings can be measured in weakly oriented samples, because a certain anisotropic distribution of orientations relative to the magnetic field is present, and therefore the residual dipolar coupling constant can be described by considering the 
time and ensemble averaging of all sampled orientations as:

$$
D_{i j}=-\frac{\mu_{0} \gamma_{i} \gamma_{j} \hbar}{4 \pi^{2} r_{i j}^{3}}\left\langle\frac{3 \cos ^{2} \theta_{i j}-1}{2}\right\rangle .
$$

The angle $\theta_{i j}$ in the Equation 2.25 can be expressed in terms of the orientation of the molecule relative to the magnetic field $\left(\xi_{x}, \xi_{y}, \xi_{z}\right)$ and the orientation of the vector relative to the molecular frame $\left(\zeta_{x}, \zeta_{y}, \zeta_{z}\right)$, as graphically represented in Figure 2.2: [Blackledge, 2005]:

$$
\cos \theta_{i j}=\left(\begin{array}{c}
\cos \zeta_{x} \\
\cos \zeta_{y} \\
\cos \zeta_{z}
\end{array}\right) \cdot\left(\begin{array}{c}
\cos \xi_{x} \\
\cos \xi_{y} \\
\cos \xi_{z}
\end{array}\right)=\cos \zeta_{x} \cos \xi_{x}+\cos \zeta_{y} \cos \xi_{y}+\cos \zeta_{z} \cos \xi_{z}
$$

With this expression the $\left\langle 3 \cos ^{2} \theta_{i j}-1\right\rangle / 2$ term can be transformed to:

$$
\left\langle\frac{3 \cos ^{2} \theta_{i j}-1}{2}\right\rangle=\frac{3}{2}\left\langle\left(\cos \zeta_{x} \cos \xi_{x}+\cos \zeta_{y} \cos \xi_{y}+\cos \zeta_{z} \cos \xi_{z}\right)^{2}\right\rangle-\frac{1}{2}
$$

which can be further reformulated as $\left(C_{i}=\cos \zeta_{i}\right.$ and $\left.c_{i}=\cos \xi_{i}\right)$ :

$$
\begin{aligned}
\left\langle\frac{3 \cos ^{2} \theta_{i j}-1}{2}\right\rangle= & \frac{3}{2}\left[\left\langle c_{x}^{2}\right\rangle C_{x}^{2}+\left\langle c_{y}^{2}\right\rangle C_{y}^{2}+\left\langle c_{z}^{2}\right\rangle C_{z}^{2}\right] \\
& +2\left\langle c_{x} c_{y}\right\rangle C_{x} C_{y}+2\left\langle c_{x} c_{z}\right\rangle C_{x} C_{z} \\
& +2\left\langle c_{y} c_{z}\right\rangle C_{y} C_{z}-\frac{1}{2}
\end{aligned}
$$

The preferential orientation of molecules $\left(\left\langle c_{x}^{2}\right\rangle,\left\langle c_{y}^{2}\right\rangle,\left\langle c_{z}^{2}\right\rangle,\left\langle c_{x} c_{y}\right\rangle,\left\langle c_{x} c_{z}\right\rangle\right.$ and $\left.\left\langle c_{y} c_{z}\right\rangle\right)$ can be described in terms of an order tensor $\mathbf{A}$ (or alignment tensor) that has been firstly introduced by Saupe in the 1960s [Saupe, 1968]. In an arbitrary molecular frame, the relationship of the RDC and the order matrix can be expressed as:

$$
D_{i j}=-\frac{\mu_{0} \gamma_{i} \gamma_{j} \hbar}{4 \pi^{2} r_{i j}^{3}} \sum_{k, l=\{x, y, z\}} A_{k l} \cos \zeta_{k} \cos \zeta_{l}
$$

with

$$
A_{k l}=\left\langle\frac{3 \cos \xi_{k} \cos \xi_{l}-\delta_{k l}}{2}\right\rangle,
$$

in which $\delta_{k l}$ is the Kronecker delta with $\delta_{k l}=1$ for $k=l$ and $\delta_{k l}=0$ for $k \neq l$, and the alignment tensor $\mathbf{A}$ is a symmetric and traceless matrix, where five independent elements determine the tensor. Using an Euler rotation $R(\alpha, \beta, \gamma)$ one can transform the molecular frame into the principal axis system, in which only the diagonal elements of the alignment tensor $\left(A_{x x}, A_{y y}, A_{z z}\right)$ are non-zero. Using the convention 
$\left|A_{x x}\right| \leq\left|A_{y y}\right| \leq\left|A_{z z}\right|$, the axial and rhombic components of the alignment tensor are defined as follows:

$$
\begin{aligned}
& A_{a}=\frac{A_{z z}}{2}, \\
& A_{r}=\frac{A_{x x}-A_{y y}}{3} .
\end{aligned}
$$

The relationship between the residual dipolar coupling constant and $A_{a}, A_{r}$ can be expressed in terms of the polar angles of the inter-spin vector $[\theta, \phi]$, as depicted in Figure 2.2:

$$
D_{i j}=-\frac{\mu_{0} \gamma_{i} \gamma_{j} \hbar}{8 \pi^{2} r_{i j}^{3}}\left[A_{a}\left(3 \cos ^{2} \theta-1\right)+\frac{3}{2} A_{r} \sin ^{2} \theta \cos 2 \phi\right] .
$$

Another useful parameter which describes the deviation from axially symmetric ordering of the alignment tensor is the asymmetry parameter $(\eta)$ [Kramer et al., 2004]:

$$
\eta=\frac{A_{x x}-A_{y y}}{A_{z z}}
$$
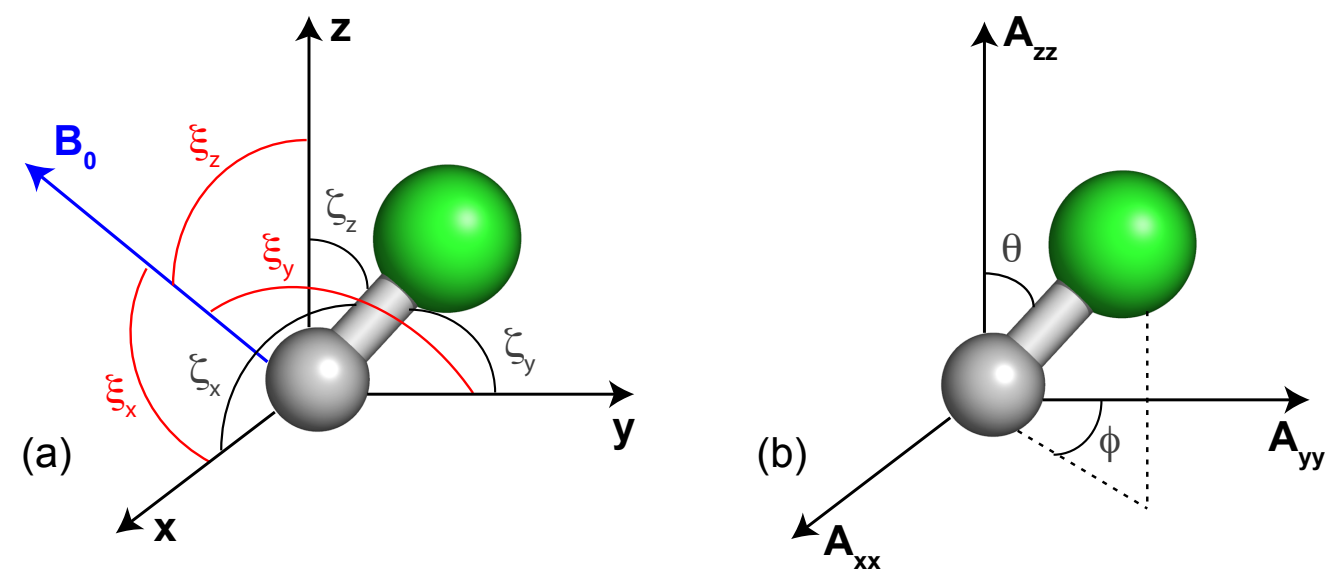

Figure 2.2: Schematic representations of (a) the orientation of the molecular frame with respect to the magnetic field $B_{0}\left(\xi_{x}, \xi_{y}, \xi_{z}\right)$ and the orientation of the inter-spin vector relative to the axes of the molecular frame $\left(\zeta_{x}, \zeta_{y}, \zeta_{z}\right)$, (b) the polar angles of the inter-spin vector $(\theta$ and $\phi)$ in the principal axis frame.

In practice, to determine the five independent components of the alignment tensor, one sets up first a linear equation in the form of [Losonczi et al., 1999]

$$
\mathbf{a x}=\mathbf{b}
$$

with 


$$
\begin{aligned}
& =\left(\begin{array}{ccccc}
\cos ^{2} \zeta_{y}^{1}-\cos ^{2} \zeta_{x}^{1} & \cos ^{2} \zeta_{z}^{1}-\cos ^{2} \zeta_{x}^{1} & 2 \cos \zeta_{x}^{1} \cos \zeta_{y}^{1} & 2 \cos \zeta_{x}^{1} \cos \zeta_{z}^{1} & 2 \cos \zeta_{y}^{1} \cos \zeta_{z}^{1} \\
\cos ^{2} \zeta_{y}^{2}-\cos ^{2} \zeta_{x}^{2} & \cos ^{2} \zeta_{z}^{2}-\cos ^{2} \zeta_{x}^{2} & 2 \cos \zeta_{x}^{2} \cos \zeta_{y}^{2} & 2 \cos \zeta_{x}^{2} \cos \zeta_{z}^{2} & 2 \cos \zeta_{y}^{2} \cos \zeta_{z}^{2} \\
\cos ^{2} \zeta_{y}^{3}-\cos ^{2} \zeta_{x}^{3} & \cos ^{2} \zeta_{z}^{3}-\cos ^{2} \zeta_{x}^{3} & 2 \cos \zeta_{x}^{3} \cos \zeta_{y}^{3} & 2 \cos \zeta_{x}^{3} \cos \zeta_{z}^{3} & 2 \cos \zeta_{y}^{3} \cos \zeta_{z}^{3} \\
\cos ^{2} \zeta_{y}^{4}-\cos ^{2} \zeta_{x}^{4} & \cos ^{2} \zeta_{z}^{4}-\cos ^{2} \zeta_{x}^{4} & 2 \cos \zeta_{x}^{4} \cos \zeta_{y}^{4} & 2 \cos \zeta_{x}^{4} \cos \zeta_{z}^{4} & 2 \cos \zeta_{y}^{4} \cos \zeta_{z}^{4} \\
\cdot & \cdot & \cdot & \cdot & \cdot \\
\cdot & \cdot & \cdot & \cdot & \cdot \\
\cdot & \cdot & \cdot & \cdot & \cdot \\
\cos ^{2} \zeta_{y}^{n}-\cos ^{2} \zeta_{x}^{n} & \cos ^{2} \zeta_{z}^{n}-\cos ^{2} \zeta_{x}^{n} & 2 \cos \zeta_{x}^{n} \cos \zeta_{y}^{n} & 2 \cos \zeta_{x}^{n} \cos \zeta_{z}^{n} & 2 \cos \zeta_{y}^{n} \cos \zeta_{z}^{n}
\end{array}\right), \\
\mathbf{x}=\left(\begin{array}{c}
D_{r e d}^{1} \\
D_{r e d}^{2} \\
D_{r z}^{3} \\
A_{x y} \\
A_{x z} \\
A_{y z}
\end{array}\right), \quad \mathbf{b}=\left(\begin{array}{l} 
\\
\cdot \\
\cdot \\
\cdot \\
D_{r e d}^{n}
\end{array}\right), &
\end{aligned}
$$

where $D_{r e d}^{i}=D_{i} \frac{r_{i j}^{3}}{\kappa}$ is the reduced RDC, $\kappa$ is defined as $\kappa=-\frac{3}{8 \pi^{2}} \gamma_{i} \gamma_{j} \mu_{0} \hbar$. This linear equation can be either solved by the standard least-square optimization algorithm or by the singular value decomposition (SVD) method. The accuracy of the solution of the determined linear system with the SVD method can be evaluated by the condition number $\kappa$, which is defined as follows:

$$
c=\|\mathbf{a}\|\left\|\mathbf{a}^{-\mathbf{1}}\right\|
$$

The linear system can be well determined, if the condition number is close or equal to 1 .

To compare two different alignment tensors, two mostly utilized parameters are the generalized degree of order (GDO) and the 5D angle of two tensors $\left(\mathbf{A}^{(\mathbf{1})}\right.$ and $\left.\mathbf{A}^{(\mathbf{2})}\right)$. GDO is independent of the molecular frame and represents the degree of the alignment. The 5D angle $\beta$ describes the orientational difference of two tensors. They are defined as follows [Kramer et al., 2004]:

$$
\begin{aligned}
\mathrm{GDO} & =\sqrt{\frac{3}{2}}|\mathbf{A}|, \\
\cos \beta & =\frac{\left\langle\mathbf{A}^{(\mathbf{1})} \mid \mathbf{A}^{(\mathbf{2})}\right\rangle}{\left|\mathbf{A}^{(\mathbf{1})}\right|\left|\mathbf{A}^{(\mathbf{2})}\right|},
\end{aligned}
$$


with

$$
\begin{aligned}
|\mathbf{A}| & =\sqrt{\sum_{k, l=\{x, y, z\}} A_{k l}^{2}}, \\
\left\langle\mathbf{A}^{(\mathbf{1})} \mid \mathbf{A}^{(\mathbf{2})}\right\rangle & =\sum_{k, l=\{x, y, z\}}\left(A_{k l}^{(1)}\right)\left(A_{k l}^{(2)}\right) .
\end{aligned}
$$

\subsubsection{J-coupling}

Compared to the direct dipolar coupling through space, the $J$-coupling arises from an indirect interaction mediated by the electrons of the bond between two nuclei. It provides local structural information such as dihedral angles [Karplus, 1963] and in some cases the information about hydrogen bonds [Kawahara et al., 2003]. The Hamiltonian of the $J$-coupling for two spins is given by [Ernst et al., 2004]:

$$
\mathscr{H}_{J}=2 \pi \mathbf{I}_{\mathbf{i}} \mathbf{J}_{\mathbf{i j}} \mathbf{I}_{\mathbf{j}}
$$

where $\mathbf{I}_{\mathbf{i}}$ and $\mathbf{I}_{\mathbf{j}}$ are the spin angular momentum operators of the spins $i$ and $j$, and $\mathbf{J}_{\mathbf{i j}}$ is the scalar coupling tensor, which can be separated into isotropic and anisotropic parts. The isotropic part is called scalar coupling and can be measured directly from the spectrum, whereas the anisotropic part cannot be easily distinguished from the dipolar coupling, however it can be neglected in the context of high-resolution liquid NMR. As briefly discussed in the introduction, this fact turns out to be an advantage in experiments for the measurement of the residual dipolar couplings, because there is no need to separate the anisotropic part of the $J$-couplings from the dipolar couplings, when a molecule is aligned in a weakly oriented medium.

For most heteronuclear and many homonuclear $J$-couplings the frequency difference $\left|\omega_{i}-\omega_{j}\right|$ fulfills the following criterion [Keeler, 2010]:

$$
\sin 2 \theta=\frac{J_{i j}}{\sqrt{\left(\omega_{i}-\omega_{j}\right)^{2}+J_{i j}^{2}}} \approx 0,
$$

which is called the weak coupling regime and the angle $\theta$ is defined as the strong coupling parameter. Under the weak coupling condition the Hamiltonian of the scalar coupling can be simplified to:

$$
\mathscr{H}_{J}=2 \pi I_{i z} J_{i j} I_{j z}
$$

in which the $I_{i z}$ and $I_{j z}$ are the spin angular momentum operators of the spins $i$ and $j$ along the $\mathrm{z}$ axis and $J_{i j}$ is the scalar coupling constant. However, many coupled 
spin systems, especially the protons in methylene groups, do not fulfill this condition. In such so-called strong coupled spin systems, the intensities and positions of the signals are affected, leading to a distortion of the multiplet. Analyzing the strong coupling effects can be in general facilitated by the numerical simulation of the spin system of interest.

One bond heteronuclear $J$-couplings vary from 100 to $300 \mathrm{~Hz}$ for different groups of chemical compounds due to their high dependency on the electronic environment. Two and three bonds homo- and heteronuclear couplings are more sensitive to the dihedral angles of the spins, whose relationship has been rationalized by Karplus [Karplus, 1959, Karplus, 1963] and Altona [Haasnoot et al., 1980] using the empirical methods:

$$
\begin{gathered}
{ }^{3} J(\phi)=A \cos ^{2} \phi+B \cos \phi+C \\
{ }^{3} J(\phi)=P_{1} \cos ^{2} \phi+P_{2} \cos \phi+P_{3}+\sum \Delta \chi_{1}\left\{P_{4}+P_{5} \cos ^{2}\left(\xi \phi+P_{6}|\Delta \chi|\right)\right\} .
\end{gathered}
$$

$\phi$ is the dihedral angle; $A, B, C$, and $P_{1}-P_{6}$ are semi-empirical constants that depend upon molecule classes; $\Delta \chi$ is the sum of the electronegativity differences between the substituents; $\xi$ stands for +1 or -1 according to the orientation of the substituent.

\subsubsection{Quadrupolar coupling}

Nuclei with $I>\frac{1}{2}$ exhibit a non-spherically electric charge distribution, and thus possess a nuclear electric quadrupole moments $Q$. The interaction of the quadrupole moment with the electric-field gradient (EFG) generated by their surroundings is called quadrupolar coupling. In the measurement of the partially aligned sample, the splitting of the deuterium signal arises from the quadrupolar coupling of the deuterium nuclei. The corresponding Hamiltonian of the quadrupolar coupling can be expressed as [Ernst et al., 2004]:

$$
\mathscr{H}_{Q}=\mathbf{I}_{\mathbf{i}} \mathbf{Q}_{\mathbf{i}} \mathbf{I}_{\mathbf{i}}
$$

in which $\mathbf{Q}_{\mathbf{i}}$ is the quadrupolar coupling tensor. In terms of the electric field gradient tensor $\mathbf{V}_{\mathbf{i}}$ at the site of the nucleus $i$ the Hamiltonian of quadrupolar coupling is given by:

$$
\mathscr{H}_{Q}=\frac{e Q_{i}}{2 I_{i}\left(2 I_{i}-1\right) \hbar} \mathbf{I}_{\mathbf{i}} \mathbf{V}_{\mathbf{i}} \mathbf{I}_{\mathbf{i}}
$$

where $\mathbf{V}_{\mathbf{i}}$ is a second-rank symmetrical tensor and $Q_{i}$ is the nuclear quadrupole moment of nucleus $i$. Under the high-field approximation, two different orders of quadrupolar coupling interaction can be distinguished. The first order of the quadrupolar coupling interaction, which splits the signal into a $2 I-1$ multiplet, can 
be averaged out under the isotropic tumbling condition. The second-order coupling that is not removed by high molecular tumbling, only needs to be considered in case the quadrupolar coupling is very strong. The splitting of the deuterium signal in the partially aligned sample is the consequence of the first-order quadrupolar coupling, which is a useful indicator as it can reflect the degree of the alignment in the medium. All equations derived for the dipolar couplings are in principle also valid for the quadrupolar couplings. 


\section{Chapter 3}

\section{Materials and Methods}

\subsection{Materials}

\subsubsection{Equipment}

In the following, all NMR spectrometers that were used during this thesis are listed in Table 3.1.

\subsubsection{Important chemicals}

Important chemicals used in this thesis are listed in Table 3.2.

\subsubsection{Software packages}

All software packages used for processing and analyzing NMR spectra, for molecular and quantum mechanics calculations, for RDC and NOE analysis and for molecular visualization and manipulation are listed in Table 3.3. 
Table 3.1: NMR spectrometers

\begin{tabular}{lll}
\hline $\begin{array}{l}\text { Field strength } \\
(\mathrm{MHz})\end{array}$ & Console & Probe head \\
\hline 400 & AVANCE & $5 \mathrm{~mm}$ PA-TCI with z-gradient \\
600 & AVANCE III & $5 \mathrm{~mm}$ QXI with z-gradient \\
600 & AVANCE & $5 \mathrm{~mm}$ cryo CP-QCI with z-gradient \\
700 & AVANCE & $5 \mathrm{~mm}$ cryo CP-TCI with z-gradient \\
700 & AVANCE III & $5 \mathrm{~mm}$ PA-TXI with z-gradient \\
& & $1.7 \mathrm{~mm}$ PA-TXI with z-gradient \\
800 & AVANCE III & $5 \mathrm{~mm}$ cryo CP-TCI with z-gradient \\
& & $1.7 \mathrm{~mm}$ cryo CP-TCI with z-gradient \\
900 & AVANCE & $5 \mathrm{~mm}$ cryo CP-TCI with z-gradient \\
\hline
\end{tabular}

Table 3.2: Important chemicals

\begin{tabular}{ll}
\hline Chemical & Company \\
\hline$N, N$-Dimethylacrylamide & Sigma-Aldrich, Germany \\
$N, N$-Methylenebisacrylamide & Sigma-Aldrich, Germany \\
2-Acrylamido-2-methylpropane & Sigma-Aldrich, Germany \\
1-sulfonic acid (AMPS) & \\
Ammonium persulfate & Sigma-Aldrich, Germany \\
$1 \mathrm{M} \mathrm{HCl}$ & Merck, Germany \\
$99.8 \% \mathrm{DMSO}-d_{6}$ & Deutero GmbH, Germany \\
$99.96 \% \mathrm{DMSO}_{6}$ & Deutero GmbH, Germany \\
\hline
\end{tabular}


Table 3.3: Software packages

\begin{tabular}{ll}
\hline Software & Reference/Origin \\
\hline Topspin 2.1 & Bruker, Germany \\
Sparky 3.110 & T. D. Goddard and D. G. Kneller, \\
& University of California, USA \\
& http://www.cgl.ucsf.edu/home/sparky \\
InsightII & Biosym Technologies, USA \\
Discover & Biosym Technologies, USA \\
Discover Studio 2.5 & Accelrys, USA \\
Mspin & Mestrelab Research SL, Spain \\
& http://www.mestrelab.com \\
PALES & [Zweckstetter and Bax, 2000] \\
& [Zweckstetter, 2008] \\
Gaussian03 & [Frisch et al., a] \\
Gaussian09 & [Frisch et al., b] \\
relax & [d'Auvergne and Gooley, 2008b] \\
& [d'Auvergne and Gooley, 2008a] \\
GROMOS & [Scott et al., 1999, Christen et al., 2005] \\
MOLMOL 2k.1 & [Koradi et al., 1996] \\
PyMOL & http://www.pymol.org \\
Open Babel & [O'Boyle et al., 2011] \\
SciPy & http://www.SciPy.org \\
\hline
\end{tabular}




\subsection{Methods}

\subsubsection{NMR spectroscopy}

All NMR experiments were acquired on the spectrometers that are listed in Table 3.1. All spectra were recorded at $25^{\circ} \mathrm{C}$. The processing of the spectra was performed using TOPSPIN 2.1 and analyzed using Sparky 3.110 or TOPSPIN 2.1 (see Table $3.3)$.

Resonance assignments were achieved using 1D and 2D NMR experiments, such as $1 \mathrm{D}-{ }^{1} \mathrm{H}, 1 \mathrm{D}-{ }^{13} \mathrm{C}$, COSY [Aue et al., 1976], TOCSY [Braunschweiler and Ernst, 1983, Cavanagh and Rance, 1990], NOESY [Jeener et al., 1979, Wider et al., 1984], ROESY [Bothner-By et al., 1984, Bax and Davis, 1985], $\left[{ }^{1} \mathrm{H},{ }^{13} \mathrm{C}\right]-\mathrm{HSQC}$ [Bodenhausen and Ruben, 1980, Palmer et al., 1991] and $\left[{ }^{1} \mathrm{H},{ }^{13} \mathrm{C}\right]-\mathrm{HMBC}$ [Bax and Summers, 1986, Cicero et al., 2001]. Unless stated otherwise, standard Bruker sequences were used to acquire the spectra. The information about interproton distances was extracted from ROESY or NOESY experiments. In some cases, a NOESY experiment with the suppression of zero-quantum interference [Thrippleton and Keeler, 2003] was utilized, so that the unwanted zero-quantum coherence did not contribute to the spectrum and the NOEs could be analyzed with high accuracy. Long-range homonuclear proton couplings were measured from $1 \mathrm{D}-{ }^{1} \mathrm{H}$, E.COSY [Griesinger et al., 1985, Griesinger et al., 1987] or P.E.COSY [Mueller, 1987] spectra, whereas long-range heteronuclear proton carbon couplings were derived with $\left[{ }^{1} \mathrm{H}-\right.$ ${ }^{13} \mathrm{C}$-HECADE [Kozminski and Nanz, 1997], $\left[{ }^{1} \mathrm{H}-{ }^{13} \mathrm{C}\right.$ ]-HSQMBC [Williamson et al., 2000] and robust-HMBC [Verdier et al., 2003] experiments.

For the RDC measurements, $\mathrm{t}_{2}$-coupled $\left[{ }^{1} \mathrm{H},{ }^{13} \mathrm{C}\right]$-HSQC or $\left[{ }^{1} \mathrm{H},{ }^{13} \mathrm{C}\right]$-CLIP-HSQC [Enthart et al., 2008] spectra were recorded for the isotropic and anisotropic samples, respectively. The pulse program of the $\left[{ }^{1} \mathrm{H},{ }^{13} \mathrm{C}\right]$-CLIP-HSQC experiment has been implemented in Bruker format and is given in the Appendix C. ${ }^{1} D_{\mathrm{CH}}$ couplings were extracted by measuring the splitting difference between isotropic and anisotropic signals.

The most frequently used solvent in this thesis was DMSO- $d_{6}$. Since above $18^{\circ} \mathrm{C}$ it is a highly hygroscopic liquid, an overwhelming $\mathrm{H}_{2} \mathrm{O}$ resonance could be observed in the proton-detected spectra. In some cases when only very limited amount of sample was available, it was necessary to seal the NMR glass tubes after the injection of the sample solution. Since the glass of the $1.7 \mathrm{~mm}$ tube is very difficult to be sealed, the plastic cap of the capillary tubes was sealed instead. 


\section{Preparation of the PH-gels as alignment media}

2-acrylamido-2-methylpropane 1-sulfonic acid (1 M), N,N-dimethylacrylamide (1 M), $N, N$-methylenebisacrylamide $(0.03 \mathrm{M})$, and ammonium persulfate $(8 \mathrm{mM})$ were dissolved in purified water followed by a devolatilization in vacuum for $15 \mathrm{~min}$. The stock solution was inserted into a gel cylinder with an inner diameter of 2 or $5.4 \mathrm{~mm}$ (Figure 3.1a), which are used for the 1.7 and $5 \mathrm{~mm}$ NMR tubes (Figure 3.1c), respectively. The material of the cylinder is polyether ether ketone (PEEK). The polymerization took 8-9 min for a gel cylinder with $2 \mathrm{~mm}$ diameter and 11-12 $\mathrm{min}$ for $5.4 \mathrm{~mm}$ at $70{ }^{\circ} \mathrm{C}$. Longer times of polymerization made the gels very fragile. The gels were washed twice with aqueous $\mathrm{HCl}(0.02 \mathrm{M})$, three times with purified water (each time for 1-2 h) and finally dried at ambient condition (air + room temperature) for at least 1 day. A comparison of the dried polymer sticks for 1.7 and $5 \mathrm{~mm}$ NMR tubes is shown in Figure 3.1b. The compounds of interest were dissolved in 450-600 $\mu \mathrm{L} \mathrm{DMSO-} d_{6}$ for $5 \mathrm{~mm}$ PH-gels and 40-60 $\mu \mathrm{L} \mathrm{DMSO-} d_{6}$ for $1.7 \mathrm{~mm}$ gels. First the dried PH-gel was placed at the bottom of the NMR tube and the prepared solution was added subsequently on top of the gel. The swelling of the PH-gels in DMSO solvent took 2-4 weeks until the gels reached an equilibrium state, which can be monitored by observation of the ${ }^{2} \mathrm{D}$ quadrupolar splitting of the deuterium signal. If the ${ }^{2} \mathrm{D}$ splitting is homogeneous and does not increase any more during time, the prepared aligned sample is ready to be measured.

\section{Preparation of the PAN-gels as alignment media}

The dried PAN sticks were prepared in the group of Prof. Burkhard Luy (Institut für Organische Chemie, Karlsruher Institut für Technologie, Karlsruhe, Germany), using the same protocol as published in [Kummerlöwe et al., 2007]. 500-650 $\mu \mathrm{L}$ of DMSO- $d_{6}$ were added to a $5 \mathrm{~mm}$ standard NMR tube together with a dried PAN polymer stick. The gel was swelled until it reached the equilibrium state, which can be monitored by observing the ${ }^{2} \mathrm{D}$ quadrupolar splitting of the solvent signal. The compounds of interest were dissolved in $50 \mu \mathrm{L}$ DMSO- $d_{6}$ and added on top of the gel, which was further allowed to equilibrate during 3-4 weeks at room temperature.

\subsubsection{Circular dichroism (CD)}

CD spectra were recorded at room temperature using a Jasco-J-810 CD spectrometer in the group of Dr. Lars Kuhn (European Neuroscience Institute, Göttingen, 

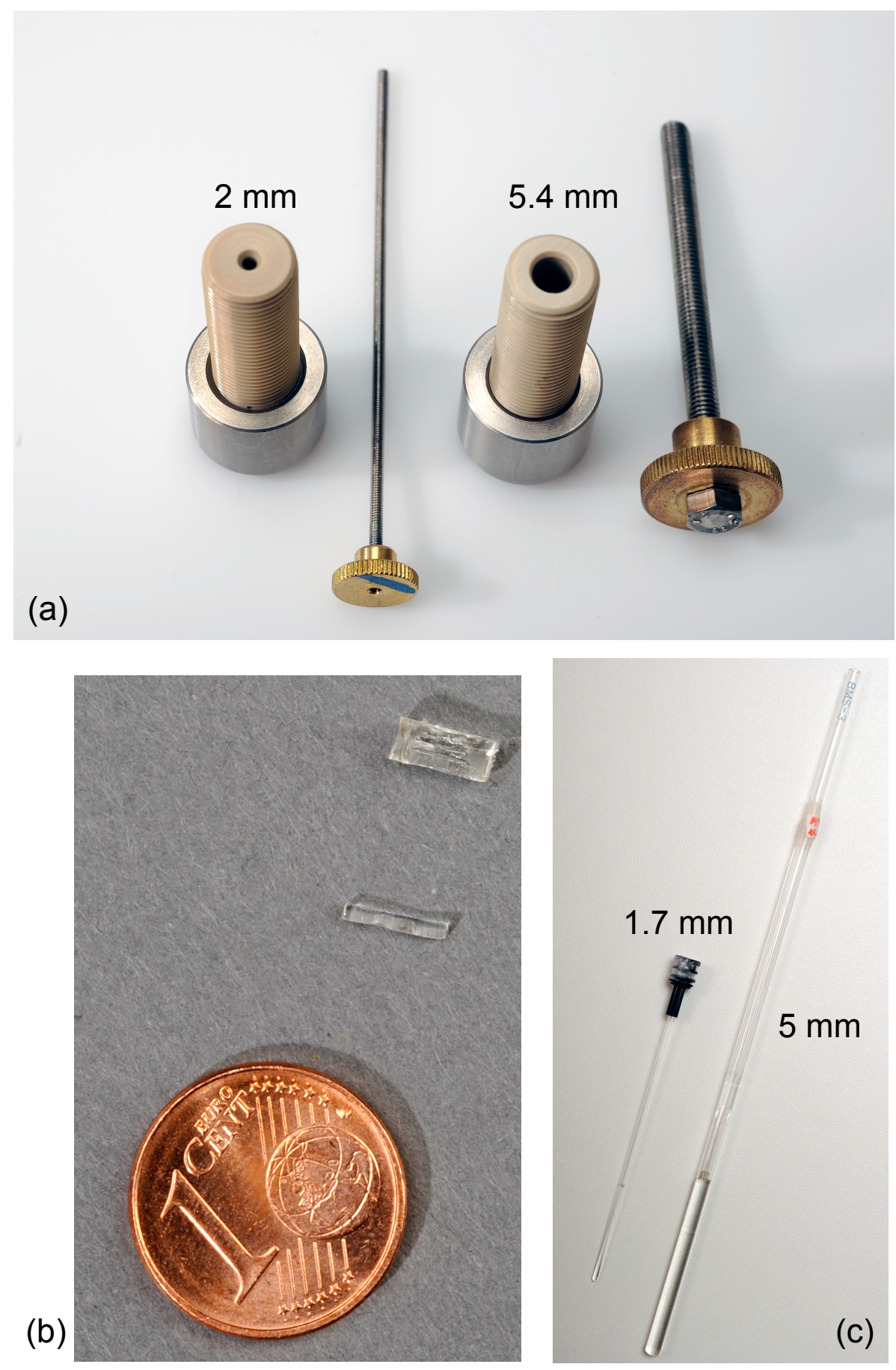

Figure 3.1: Schematic illustration of the preparations and usages of the PH-gels for NMR tubes with different sizes: (a) Apparatus for preparing PH-gels for 1.7 and $5 \mathrm{~mm} \mathrm{NMR}$ tubes. (b) Comparison of the dried gels for 1.7 and $5 \mathrm{~mm}$ NMR tubes. (c) Swelling gels with sample in 1.7 and $5 \mathrm{~mm}$ NMR tubes.

Germany). $200 \mu \mathrm{L}$ of $20-30 \mu \mathrm{M}$ sample dissolved in DMSO solvent was measured using a $0.1 \mathrm{~cm}$ path length cuvette. The spectra were acquired from $250 \mathrm{~nm}$ to 
$400 \mathrm{~nm}$ with $0.5 \mathrm{~nm}$ resolution at a scan-rate of $20 \mathrm{~nm} / \mathrm{min}$.

\subsubsection{Optical rotatory dispersion (ORD)}

Optical rotations were measured at $22-25{ }^{\circ} \mathrm{C}$ using a $2 \mathrm{~mL}$ cuvette at $589 \mathrm{~nm}, 578 \mathrm{~nm}$, $546 \mathrm{~nm}$ and $436 \mathrm{~nm}$ with a Perkin Elmer model 241 polarimeter. All the measurements were performed by the coworkers of Prof. Michael Reggelin (Clemens-SchöpfInstitut für Organische Chemie und Biochemie, Technische Universität Darmstadt, Darmstadt, Germany).

\subsubsection{Molecular dynamics (MD) simulations and analysis}

\section{Explicit solvent molecular dynamics simulations}

Explicit solvent molecular dynamics simulations were performed with the GROMOS96 software package [Scott et al., 1999]. The force field parameters for the systems were taken from the 53A6 GROMOS united atom force field [Oostenbrink et al., 2004] whereas the topology of the molecules was constructed manually. After minimization in vacuum the molecule was solvated in a DMSO solvent box using the standard GROMOS96 DMSO solvent model. For charged molecules, counter ions were solvated in the DMSO solvent box as well. The center of mass of the molecule was placed in the center of the box. The energy of the whole system was first minimized using the conjugate gradient method. After 100 ps of equilibration at $298 \mathrm{~K}$, simulations were performed at constant temperature $(298 \mathrm{~K})$ and pressure (1 atm).

\section{Conformational clustering}

For the chosen trajectory, the root-mean-square difference (RMSD) of the atomic coordinates between pairs of structures was calculated after superimposing all carbon atoms. Two conformations were put into the same cluster if their RMSD was below a threshold of $0.2 \mathrm{~nm}$. The central member structure, defined as the structure with the highest number of neighbors in one cluster, is defined as the representative conformation of this cluster. The population of each cluster was determined by the number of conformations contained in the cluster.

\section{Molecular dynamics simulations in vacuo}

Molecular dynamics simulations for [Sun et al., 2011a] were performed in vacuo with the Discover software using the consistent valence force field (CVFF) [Hagler 
et al., 1974, Hagler et al., 1979], a standard force field for small molecules. The MD simulations were carried out at $298 \mathrm{~K}$ for $1 \mathrm{~ns}$.

\subsubsection{Molecular mechanics simulations}

\section{Simulated annealing structural calculations}

Simulated annealing structural calculations for [Sun et al., 2011b] were performed with the Discover software using the CFF91 force field [Maple et al., 1988]. The starting structures were firstly minimized in vacuo, using the steepest descent algorithm. The simulated annealing protocol comprised an initial molecular dynamics procedure at $298 \mathrm{~K}$ to obtain 200 structures. Then each of these 200 structures was heated to a temperature of $600 \mathrm{~K}$ and equilibrated at this temperature for 5 ps. They were then slowly cooled down to $300 \mathrm{~K}, 100 \mathrm{~K}$ and finally to $3 \mathrm{~K}$. At each temperature step the molecule was equilibrated for 5 ps. At the end of simulated annealing, all structures were energy minimized using steepest descent followed by the conjugate gradient algorithm.

\section{Conformational search}

Conformational search based on molecular mechanics calculations was performed with Discovery Studio 2.5 using the standard CFF force field [Lifson and Warshel, 1968]. In the program we used the "generate conformations" protocol with the "FAST" or "BEST" search methods. Each new generated conformation is optimized in the torsion angle space with pooling penalty to maintain conformational diversity.

\subsubsection{Density functional theory (DFT) calculations}

\section{Structure optimizations}

Structure optimizations by DFT were performed with Gaussian03 or Gaussian09 (keyword $=\mathrm{opt}$ ). All the calculations were performed with the integral equation formalism variant polarizable continuum model (IEFPCM).

\section{Energy and free energy calculations}

Energy $(E)$ and free energy (or free enthalpy $G$ ) were calculated with Gaussian03 or Gaussian09 (keyword = freq). All the calculations were performed with the integral equation formalism variant polarizable continuum model (IEFPCM). The energy and free energy were calculated at $298 \mathrm{~K}$ and 1 atm. The equations used for calculating energy and free energy are given in Thermochemistry in Gaussian 
(www.gaussian.com/g_whitepap/thermo.htm). For convenience, the most important equations for calculating these thermodynamic properties are given in Appendix B.

\section{Circular dichroism computations}

Time-dependent DFT (TD-DFT) [Berova et al., 2007, Petrovic et al., 2010] was used to calculate the spin-allowed excitation energies, rotatory $\left(R_{n}\right)$ and oscillator strengths $\left(f_{n}\right)$ of the lowest 50 excited states (keyword: $t d=($ nstates $=50)$ ). The calculations were carried out with Gaussian03 or Gaussian09 with the IEFPCM solvent continuum model. The peak intensity $\Delta \epsilon(\nu)$ was calculated via the following relation [Petrovic et al., 2010]:

$$
\Delta \epsilon(\nu)=\frac{R_{i} \nu_{i}}{2.296 \times 10^{-39} \sqrt{\pi} \sigma} \exp \left[-\left(\frac{\nu-\nu_{i}}{\sigma}\right)^{2}\right]
$$

where $\sigma$ is the bandwidth in $\mathrm{eV}, \nu$ the frequency in $\mathrm{cm}^{-1}$, and $R$ the theoretical ECD response.

\section{Optical rotatory dispersion computations}

The optical rotatory dispersion calculations were performed at the four wavelengths 436, 546, 578 and $589 \mathrm{~nm}$ using the optimized structures as input coordinates (keyword: polar $=$ OptRot). The calculations were carried out with Gaussian03 or Gaussian09 in vacuo with the IEFPCM solvent continuum model.

\subsubsection{Analysis of residual dipolar couplings}

\section{RDC fitting on a single conformer}

Five linearly independent parameters of the traceless and symmetric alignment tensor A were calculated by using:

$$
D=-\frac{3 \mu_{0} \hbar \gamma_{A} \gamma_{B}}{8 \pi^{2} R^{3}} \mathbf{r}^{\mathbf{T}} \mathbf{A r}
$$

in which $\gamma_{A}$ and $\gamma_{B}$ are the gyromagnetic ratios of both interacting nuclei; $R$ is the internuclear distance; $\mathbf{r}$ is the unit norm vector connecting the nuclei; $\mathbf{A}$ is the alignment tensor. The fitting procedure of the experimental RDC data was performed using either an in-house modified version of the Mspin or the PALES program. Residual dipolar couplings and the pdb or xyz files of the selected conformers were given as the input data. Methyl and phenyl ${ }^{1} D_{\mathrm{CH}}$ couplings were treated in the 
analysis by means of the 3-fold and 2-fold jump model [Verdier et al., 2003, SánchezPedregal et al., 2009]. Five independent members of the alignment tensor were optimized using the singular value decomposition (SVD) method [Losonczi et al., 1999]. The fitting between theoretical and experimental RDCs was evaluated according to Cornilescu's $Q$ factor: [Cornilescu et al., 1998]:

$$
Q=\sqrt{\frac{\sum_{j}\left(D_{j}^{o b s}-D_{j}^{c a l c}\right)}{\sum_{j}\left(D_{j}^{o b s}\right)^{2}}},
$$

where $j$ is the index over all RDCs.

\section{Ensemble RDC fitting using single alignment tensor approach}

Under the assumption of a single alignment tensor, the relative orientation of conformers in the ensemble is determined by rotating each structure to a common frame, which can either be a rigid part of the structure or the mean structure. This procedure was performed with the program MOLMOL2k.1 or an in-house modified version of the Mspin program using the command line (world.superimpose"a,b-d" (a,b,d are atom numbers)). Five linearly independent parameters of the traceless and symmetric alignment tensor $\mathbf{A}$ were calculated by using:

$$
\langle D\rangle=-\sum_{i=1}^{N} p_{i} \frac{3 \mu_{0} \hbar \gamma_{A} \gamma_{B}}{8 \pi^{2} R^{3}} \mathbf{r}_{\mathbf{i}}^{\mathbf{T}} \mathbf{A r}_{\mathbf{i}}
$$

in which $p_{i}$ is the population; $\mathbf{r}_{\mathbf{i}}$ is the unit norm vector of the $i$ th conformer; $\mathbf{A}$ is the common alignment tensor for all conformers. The fitting procedure of the experimental RDC data was performed using either an in-house modified version of the Mspin or relax program. Residual dipolar couplings and the pdb or xyz files of the selected conformers were given as the input data. Methyl and phenyl ${ }^{1} D_{\mathrm{CH}}$ couplings were treated in the analysis by means of the 3-fold and 2-fold jump model [Verdier et al., 2003, Sánchez-Pedregal et al., 2009].

Five independent members of the alignment tensor were optimized either using the singular value decomposition (SVD) method [Losonczi et al., 1999] as implemented in the Mspin program or a simplex algorithm that was implemented in the relax program. In Mspin, the population of each structure can be simultaneously determined using a combination of grid-search and a constrained least-squared Levenberg-Marquardt procedure as previously reported [Sánchez-Pedregal et al., 2009]. The theoretical RDCs were calculated from the obtained alignment tensor. The fitting between theoretical and experimental RDCs was evaluated according to 
Cornilescu's $Q$ factor:

$$
Q=\sqrt{\frac{\sum_{j}\left(D_{j}^{o b s}-\overline{D_{j}^{c a l c}}\right)}{\left(\sum_{j} D_{j}^{o b s}\right)^{2}}},
$$

where $j$ is the index over all RDCs and the back-calculated $\overline{D_{j}^{c a l c}}$ is averaged over all members of the ensemble.

\section{Ensemble RDC fitting using multiple alignment tensors}

In the multiple-tensor analysis the alignment tensor for each conformer was determined individually by using:

$$
\langle D\rangle=-\sum_{i=1}^{N} p_{i} \frac{3 \mu_{0} \hbar \gamma_{A} \gamma_{B}}{8 \pi^{2} R^{3}} \mathbf{r}_{\mathbf{i}}^{\mathbf{T}} \mathbf{A}^{\mathbf{i}} \mathbf{r}_{\mathbf{i}}
$$

Note that $\langle D\rangle$ depends on the product of the population $p_{i}$ and the individual alignment tensor $\mathbf{A}^{\mathbf{i}}$, and therefore the overall degree of alignment cannot be determined unless the populations are previously known, or alternatively, the populations can be determined if the size of the tensors $\left|\mathbf{A}^{\mathbf{i}}\right|$ is known [Thiele et al., 2009, PérezBalado et al., 2011]. In this thesis, the populations of the main conformers have been derived from MD simulations. The coordinates of each conformation (pdb or xyz files) and its population were given as input for back-calculation of the RDCs. The calculation of the alignment tensor for each structure performed by the SVD method was implemented using the Python programming language and the SciPy numeric library. Methyl ${ }^{1} D_{\mathrm{CH}}$ RDCs were treated by averaging of the corresponding SVD entries as previously described [Sánchez-Pedregal et al., 2009]. The linear equation for determining the alignment tensor for each conformer from the experimental RDCs using the SVD method is: 


$$
\left(\begin{array}{c}
D_{1}^{r e d} \\
D_{2}^{r e d} \\
D_{3}^{r e d} \\
\cdot \\
\cdot \\
\cdot \\
D_{n-1}^{r e d} \\
D_{n}^{r e d}
\end{array}\right)=\mathbf{M}\left(\begin{array}{c}
A_{x y}^{1} \\
A_{x z}^{1} \\
A_{y y}^{1} \\
A_{y z}^{1} \\
A_{z z}^{1} \\
\cdot \\
\cdot \\
\cdot \\
A_{x y}^{m} \\
A_{x z}^{m} \\
A_{y y}^{m} \\
A_{y z}^{m} \\
A_{z z}^{m}
\end{array}\right),
$$

where $D_{i}^{r e d}=D_{i} \frac{R^{3}}{\kappa}$ is the reduced RDC, $\kappa$ is defined as $\kappa=-\frac{3}{8 \pi^{2}} \gamma_{I} \gamma_{S} \mu_{0} \hbar, \mathbf{A}^{\mathbf{i}}$ is the alignment tensor or the $i$ th conformer and $\mathbf{M}$ is a matrix composed of the direction cosines of the internuclear vectors [Losonczi et al., 1999]. The theoretical RDCs were back-calculated from the obtained tensor and the previously determined populations from the MD simulations. We evaluated the fitting between theoretical and experimental RDCs according to the $Q$ factor (Equation 3.5).

\subsubsection{Quantitative analysis of ROE and NOE data}

NOESY or ROESY peaks were integrated using the Lorentzian fit method that has been implemented in the Sparky program. The NOE or ROE integrals were translated into interproton distances, using as a reference proton pairs with known distances, such as methylene protons $(1.78 \AA)$ or protons in aromatic rings. The violation for the ROE or NOE in $\AA^{2}$ is defined as:

$$
U= \begin{cases}\sum_{j}\left(\overline{r_{j}^{\text {calc }}}-r_{\text {min }}\right)^{2}, & \text { if } \overline{r_{j}^{\text {calc }}}<r_{\text {min }} \\ 0, & \text { if } r_{\text {min }} \leq \overline{r_{j}^{\text {calc }}} \leq r_{\max } \\ \sum_{j}\left(\overline{r_{j}^{\text {calc }}}-r_{\max }\right)^{2}, & \text { if } \overline{r_{j}^{\text {calc }}}>r_{\max }\end{cases}
$$

where $\overline{r_{j}^{\text {calc }}}$ is the back-calculated ROE or NOE distance, averaged to the $-6^{\text {th }}$ power over all members of the ensemble, and the uncertainty of the experimental NOE or ROE determines $r_{\min }$ and $r_{\max }$.

Similarly to the RDC analysis, the $Q$ factor for ROEs or NOEs is defined as: 
3.2. METHODS

$$
Q=\sqrt{\frac{U}{\sum_{j}\left(r_{j}^{o b s}\right)^{2}}} .
$$




\section{Chapter 4}

\section{Stereochemical elucidation of molecules of class A}

\subsection{Introduction}

As illustrated in Figure 1.2, molecule class A contains those molecules in which all stereogenic centers "communicate" with each other. In general, the relative configuration of these correlated stereogenic units can be determined using the conventional NMR restraints, such as NOEs and long-range $J$-couplings, or recently with the aid of quantum mechanics supported chemical shift computations. However, when the molecules of interest are either flexible or complex, sometimes ambiguities about the configuration still remain. The molecule LLG1, whose absolute configuration will be illustrated in this chapter, belongs to this class of molecules. Herein, to overcome these obstacles, we have included RDC analysis in the stereochemical investigation. The resulting relative configurational and conformational information was subsequently employed for the determination of absolute configuration by ECD spectroscopy supported by DFT computations.

In the second part of this chapter, the absolute configuration of vatiparol, a new identified resveratrol trimer, was determined by a combination of RDC- and NOE-based NMR analysis together with DFT-supported chiroptics. The precise conformation of vatiparol was established by the RDC analysis, which was essential for the correct calculation of chiroptical properties. This example demonstrates the important power of RDCs again in the configurational and conformational determination. The major difficulty of this project came from the limitation of the sample amounts and conventional methods for measuring RDCs could not be applied in this case. Herein, we decided to take advantage of microprobe NMR for the structural elucidation of natural products with low availability. The preparation of a slim PH- 
gel for $1.7 \mathrm{~mm}$ NMR tubes was introduced, so that only one milligram of vatiparol was required for the RDC measurements. Based on the RDC-determined ensemble of conformers, comparison of experimental and computed ECD and ORD spectra lead independently to the same absolute configuration of vatiparol.

The third system that will be presented in this chapter are two naphthoquinone epoxides, which are 2,3-epoxy-2,3-dihydrolapachol (naphth 1) and 2,3-epoxy-2,3dihydro-8-hydroxylapachol (naphth 2), respectively. These two molecules each contain two unknown stereocenters, nevertheless due to geometrical restrictions, only one relative configuration is possible. Chiroptical methods together with DFT computations were utilized to establish the absolute configuration of naphth 1 and naphth 2 .

\subsection{Determination of the absolute configuration of LLG1}

\subsubsection{Introduction}

LLG1 is a secondary metabolite produced by Fusarium sp.. As shown in Figure 4.1, it is a derivative of a pyrrole fatty ester, containing two long alkyl chains (C1-C11 and C14-C20). $4 \mathrm{mg}$ of LLG1 has been isolated for the first time by our collaborators Liang-Yan Liu and Prof. Ji-Kai Liu (Kunming Institute of Botany, Chinese Academy of Science, Kunming, China). The aim of our work here is to determine the absolute configuration of the two unknown stereocenters C12 and C21.

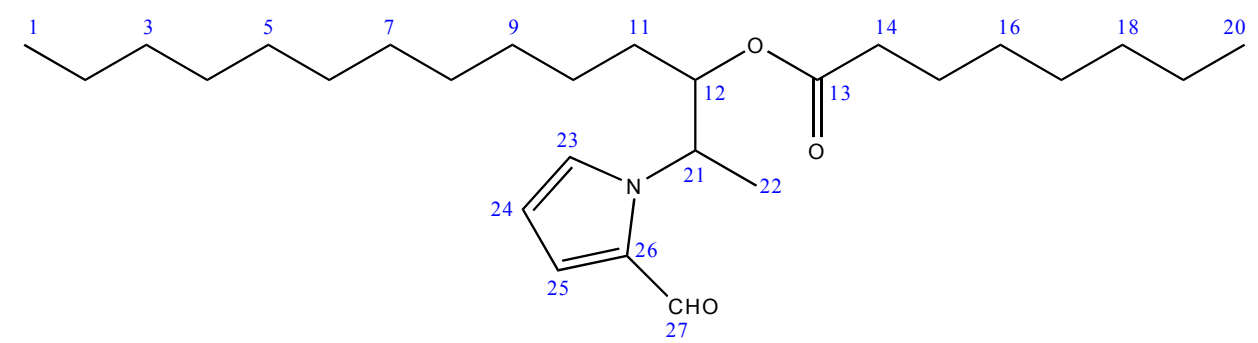

Figure 4.1: Structure and atom numbering of LLG1 
Table 4.1: Carbon and proton chemical shifts of LLG1 in DMSO- $d_{6}$ at $298 \mathrm{~K}$.

\begin{tabular}{lcll}
\hline Carbon & Chemical shift $(\mathrm{ppm})$ & Proton & Chemical shift $(\mathrm{ppm})$ \\
\hline C10 & 25.2 & H10a & 1.22 \\
& & H10b & 1.16 \\
C11 & 30.6 & H11a & 1.43 \\
& & H11b & 1.34 \\
C12 & 75.1 & H12 & 5.03 \\
C13 & 172.6 & & \\
C14 & 33.9 & H14 & 2.24 \\
C15 & 24.9 & H15 & 1.46 \\
C21 & 54.4 & H21 & 5.37 \\
C22 & 15.1 & H22 & 1.44 \\
C23 & 129.6 & H23 & 5.37 \\
C24 & 110.3 & H24 & 6.24 \\
C25 & 126.0 & H25 & 7.04 \\
C26 & 131.7 & & \\
C27 & 180.2 & H27 & 9.50 \\
\hline
\end{tabular}

\subsubsection{Elucidation of the relative configuration and conforma- tion by NMR}

\section{NMR resonance assignment}

The ${ }^{1} \mathrm{H}$ and ${ }^{13} \mathrm{C}$ chemical shifts of LLG1 in DMSO- $d_{6}$ were assigned by means of $1 \mathrm{D}-{ }^{1} \mathrm{H}, 1 \mathrm{D}-{ }^{13} \mathrm{C}$, COSY, TOCSY, NOESY, $\left[{ }^{1} \mathrm{H},{ }^{13} \mathrm{C}\right]-\mathrm{HSQC}$ and $\left[{ }^{1} \mathrm{H},{ }^{13} \mathrm{C}\right]-\mathrm{HMBC}$ NMR spectra and are listed in Table 4.1. All NMR spectra were recorded at $25{ }^{\circ} \mathrm{C}$. Due to strong chemical shift degeneracy of the alkyl chain, the assignment of the protons and carbons 1-9 and 16-20 could not be achieved.

\section{Measurement of scalar and residual dipolar couplings}

Long-range proton-proton couplings were extracted from a P.E.COSY spectrum, and long-range proton-carbon couplings were derived with $\left[{ }^{1} \mathrm{H}^{-13} \mathrm{C}\right]$-HECADE and $\left[{ }^{1} \mathrm{H}-{ }^{13} \mathrm{C}\right]-\mathrm{HSQMBC}$ experiments (Table 4.2).

For the RDC measurement, $2.3 \mu$ mol LLG1 was aligned in a PH-gel with $50 \mu \mathrm{L}$ DMSO in a $1.7 \mathrm{~mm}$ NMR tube. After 3 weeks of full swelling, a deuterium quadrupolar splitting of $13.4 \mathrm{~Hz}$ was observed and $10{ }^{1} D_{\mathrm{CH}}$ values (Table 4.3) were measured using $\left[{ }^{1} \mathrm{H}-{ }^{13} \mathrm{C}\right]-\mathrm{CLIP}-\mathrm{HSQC}$ experiments. 
Table 4.2: Long-range proton-proton and proton-carbon coupling constants of LLG1, n: no signals in the HSQMBC and HMBC spectra.

\begin{tabular}{llll}
\hline Atoms & ${ }^{3} J_{\mathrm{HH}}(\mathrm{Hz})$ & Atoms & ${ }^{2,3} J_{\mathrm{CH}}(\mathrm{Hz})$ \\
\hline H12-H11a & 3.3 & H21-C12 & -1.4 \\
H12-H11b & 9.4 & H12-C21 & -2.6 \\
& & H12-C22 & 3.0 \\
& & H12-C10 & 2.8 \\
& & H11b-C21 & $\mathrm{n}$ \\
& & H10a-C12 & 1.7 \\
& & H10b-C12 & $\mathrm{n}$ \\
& & H21-C11 & $\mathrm{n}$ \\
\hline
\end{tabular}

\section{Configurational analysis}

LLG1 is a molecule that contains a large number of potentially rotatable bonds, leading to a complex conformational space that is difficult to sample. In this study in order to simplify the problem, only the conformations stemming from the rotation of the bonds $\mathrm{C} 11-\mathrm{C} 12, \mathrm{C} 12-\mathrm{C} 21$, and $\mathrm{C} 21-\mathrm{N}$, which are close to the two unknown stereocenters, were considered. Because of a small proton-proton coupling between $\mathrm{H} 11 \mathrm{a}$ and H12 (around $3 \mathrm{~Hz}$ ) and a large coupling between H11b and H12 (around $10 \mathrm{~Hz}$ ), as shown in Figure 4.2 and Table 4.2, only two out of three conformers of the C10-C11-C12-O dihedral are possible main conformations. Since from the $J$-coupling analysis no conclusive results can be obtained regarding the major conformation of the C11-C12-C21-N dihedral, all three conformations (C11/N: + gauche, - gauche, anti) needed to be considered. Besides, two conformations for the H21-C21N-C23 dihedral (H21/C23: trans, cis) were found from DFT calculations. Taken together, $12(=2 \times 3 \times 2)$ conformations were generated for the two possible relative configurations $(12 S, 21 S)$ and $(12 S, 21 R)$, respectively, and DFT optimized at the B3LYP $/ 6-31 G(d)$ level using the IEFPCM solvent continuum model with DMSO as the solvent (Figure A.1). For all these 12 conformations of each configuration, the alkyl chain from $\mathrm{C} 1$ to $\mathrm{C} 11$ and from $\mathrm{C} 14$ to $\mathrm{C} 20$ was assumed to adopt a anti conformation, which is the energetically most preferable conformer [Nasipuri, 2012]. The important dihedral angles for defining the conformation of possible conformers 1-12 for both possible relative configurations are given in Table A.1.

After the attempt to determine the relative configuration of the two neighboring stereocenters C12 and C21 using ${ }^{2,3} J$-couplings (Table 4.2) exclusively had failed, residual dipolar couplings were utilized to probe the conformation and relative con- 


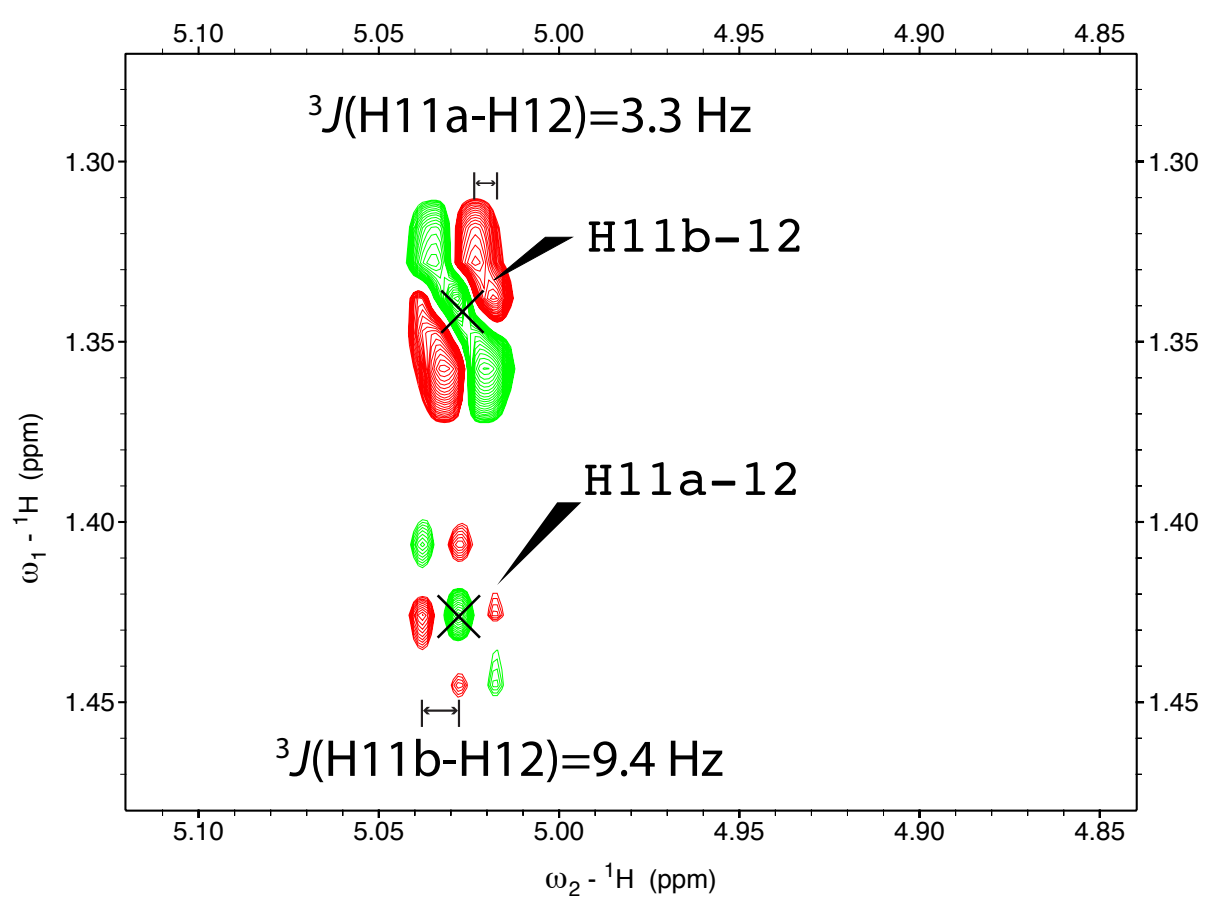

Figure 4.2: Regions from P.E.COSY spectrum showing the homonuclear couplings of H11a-H12 and H11b-H12.

figuration of LLG1. 10 RDCs were used to calculate the alignment tensor for each possible conformation using the SVD method [Losonczi et al., 1999], followed by a back-calculation of the theoretical RDCs based on the obtained alignment tensor and structural model. All calculations were performed with an in-house modified version of the program Mspin. In the RDC analysis, it was necessary to assign the two pairs of prochiral protons of $\mathrm{C} 10$ and $\mathrm{C} 11$, which has been achieved as follows: H10a was defined as the proton that is closest to H12 based on the strong NOE peak (Figure 4.6b); H11b was defined as the proton which is anti to H12 based on the large scalar couplings (Table 4.2 and Figure 4.2). The quality of the fit between the experimental RDCs and the back-calculated ones - expressed by the $Q$ factor was used to identify the correct configuration and conformation. The results summarized in Figure 4.3 clearly show that the two lowest $Q$ factors were obtained for conformations 9 and 11 of $(12 R, 21 R)$ or $(12 S, 21 S)$ with $Q$ factors of 0.20 and 0.09 , whereas the $Q$ factors of all other possible conformers of $(12 R, 21 S)$ or $(12 S, 21 R)$ are significantly higher. Furthermore, as seen in Figure A.3, no significant violation of the experimental data is observed for any $\mathrm{RDC}$ of the the best fitting conformer 11 of the relative configuration $(12 R, 21 R)$ or its enantiomer.

Conformations 9 and 11 that exhibit the lowest $Q$ factors of the RDC fitting mainly differ in the dihedral H12-C12-C21-H21 (Table A.1, Figure 4.4). According 
Table 4.3: Experimental residual dipolar couplings of LLG1 in PH-gel/DMSO together with the back-calculated ones for the best fitting conformer 11 of $(12 S, 21 S)$ or $(12 R, 21 R)$.

\begin{tabular}{lrr}
\hline CH-vector & Exp. ${ }^{1} D_{\mathrm{CH}}(\mathrm{Hz})$ & Calc. ${ }^{1} D_{\mathrm{CH}}(\mathrm{Hz})$ \\
\hline C10-H10a & 17.1 & 18.5 \\
C10-H10b & 7.6 & 7.4 \\
C11-H11a & 3.6 & 4.0 \\
C11-H11b & 12.1 & 12.5 \\
C12-H12 & 19.1 & 17.1 \\
C21-H21 & -3.4 & -3.3 \\
C22-H22 & -5.0 & -6.6 \\
C23-H23 & 18.2 & 18.1 \\
C24-H24 & -3.2 & -2.9 \\
C25-H25 & 6.2 & 6.3 \\
\hline
\end{tabular}

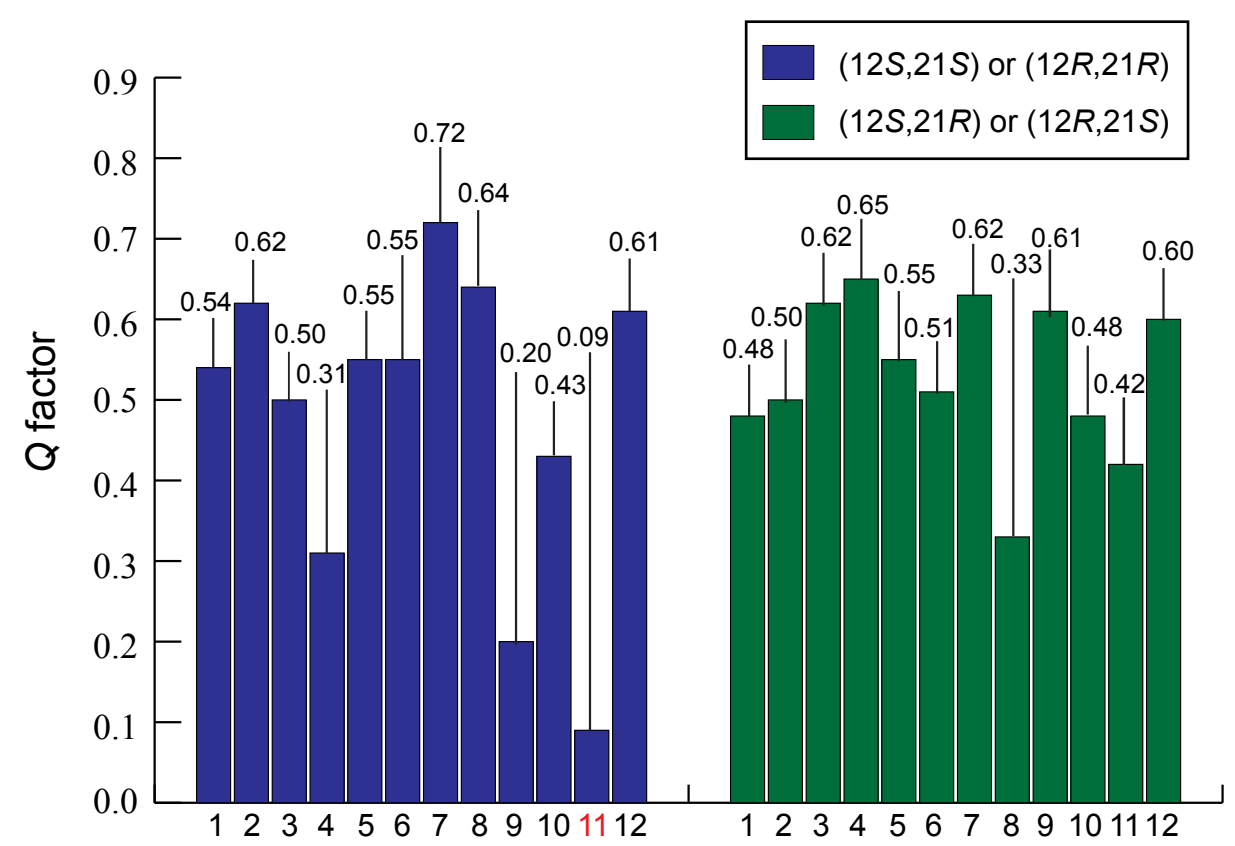

Figure 4.3: Comparison of the $Q$ factors of the RDC fitting for the two possible relative configurations $(12 S, 21 S)$ and $(12 S, 21 R)$ or their enantiomers. For each configuration 12 different conformers were considered in the RDC fitting. The conformer 11 of the configuration $(12 S, 21 S)$ or $(12 R, 21 R)$, which exhibits the significantly lowest $Q$ factor, is highlighted in red.

to [Cyr et al., 1978, Riccio et al., 2003], for dihedral angles $\mathrm{O}-\mathrm{C}_{\mathrm{A}}-\mathrm{C}_{\mathrm{B}}-\mathrm{H}$, where $\mathrm{C}_{\mathrm{B}}$ bears one oxygen or nitrogen, the observed ${ }^{2} J$ values range from -6 to $0 \mathrm{~Hz}$. In addition, the anti conformation between proton and heteroatom leads to a small value of the two bond $\mathrm{C} / \mathrm{H}$ coupling constant $\left({ }^{2} J_{\mathrm{CH}}\right)$, whereas a large coupling con- 
stant is associated with a gauche conformation. Due to the fact that small negative coupling constants for both $\mathrm{H} 21 / \mathrm{C} 12$ and $\mathrm{H} 12 / \mathrm{C} 21$, and a small positive coupling for $\mathrm{H} 12 / \mathrm{C} 22$ (Table 4.2) were observed, the anti relationship between H12 and N, as well as between $\mathrm{H} 21$ and $\mathrm{O}$ was determined as the main conformation. This is the case for conformer 11, but not for conformer 9, as shown with the aid of the Newman projections in Figure 4.4. Additionally, a NOESY spectrum with $200 \mathrm{~ms}$ mixing time was acquired and NOE peaks that are relevant in the configurational and conformational analysis have been integrated (Table 4.4). For a comparison, the corresponding interproton distances of the best RDC-fitting conformer 11 were calculated (Table 4.4). The relationship between NOE integrals and the distance to the power of minus six is in a good agreement for the following proton pairs: H12 and H10a, H22 and H23, H25 and H27, H11b and H12, H11a and H12, H21 and H27, which further supports conformer 11 as the major conformer. However, a relatively smaller NOE integral of $\mathrm{H} 10 \mathrm{~b}$ and $\mathrm{H} 21$, together with a relatively larger NOE integral of H10a and H21, were observed compared to the predicted ones. The reason for this could be a conformational averaging around the dihedral C11-C10, which nevertheless does not change the conclusion that conformer 11 was determined as the main conformation, because only dihedrals of C11-C12, C12-C21 and C21-N define the conformation. To sum up, together with the $J$-coupling analysis, the observed key NOEs as indicated by arrows in Figure 4.6 confirmed conformer 11 as the preferred main conformation. For the second best RDC-fitting conformer 9, an NOE between H23 and H10b is expected, as in the structural model the distance between these two protons is less than $2.8 \AA$. The absence of this NOE in the NOESY spectrum (Figure 4.6) excludes conformer 9 as the major conformation.

The energy and free energy of each possible conformer of the relative configuration $(12 R, 21 R)$ or $(12 S, 21 S)$ was calculated by DFT using the B3LYP/6-31G(d) basis set that has been employed in the structure optimizations as well. Comparison of the computational energy of individual conformers as shown in Table A.2 identifies the conformer $2(\Delta \mathrm{E}=0, \Delta \mathrm{G}=0)$ and NMR-determined conformer $11(\Delta \mathrm{E}=0.5$ $\left.\mathrm{kcal} \cdot \mathrm{mol}^{-1}, \Delta \mathrm{G}=1.1 \mathrm{kcal} \cdot \mathrm{mol}^{-1}\right)$ as the lowest energy and free energy conformations. However, due to a deficit agreement with the $\operatorname{RDC}$ data $(Q=0.62)$ as well as from the NOE analysis, as the expected NOE between H21 and H23 cannot be observed in the spectrum, the energetically most preferential conformation (conformer 2, see Table A.1 and Figure A.1) can be excluded to be the major conformation. Although many studies show that the NMR data can be approved by DFT-based energy calculations [Sánchez-Pedregal et al., 2009, Pérez-Balado et al., 2011], this is an example suggesting that for some systems DFT computations with the B3LYP func- 
tionals are not able to accurately predict the relative energies [Wodrich et al., 2007]. In conclusion, the relative configuration of LLG1 was established as $(12 R, 21 R)$ or $(12 S, 21 S)$ by the RDC analysis exclusively. Furthermore, disregarding the potential conformational flexibility of the alkyl chains, the preferential population of a single local conformation around the dihedrals of C11-C12, C12-C21 and C21-N (conformer 11) was determined by the excellent fit of the RDC data, and was further corroborated by the $J$-coupling and NOE analysis.

C12:R, C21:R

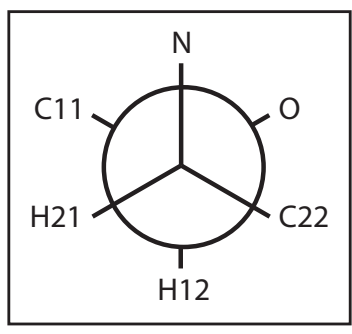

conformer 11

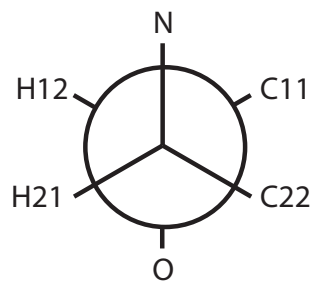

conformer 9

\begin{tabular}{lll}
\hline${ }^{3}(\mathrm{H} 12-\mathrm{H} 21)$ & very small & very weak cross peak in COSY \\
${ }^{2}(\mathrm{H} 21-\mathrm{C} 12)$ & $-1.4 \mathrm{~Hz}$ & extracted from $\left[{ }^{1} \mathrm{H}-{ }^{-13} \mathrm{C}\right]-\mathrm{HECADE}$ \\
${ }^{2} \mathrm{~J}(\mathrm{H} 12-\mathrm{C} 21)$ & $-2.6 \mathrm{~Hz}$ & extracted from $\left[{ }^{1} \mathrm{H}-{ }^{13} \mathrm{C}\right]-\mathrm{HECADE}$ \\
${ }^{3} \mathrm{~J}(\mathrm{H} 12-\mathrm{C} 22)$ & $3.0 \mathrm{~Hz}$ & extracted from $\left[{ }^{1} \mathrm{H}-{ }^{13} \mathrm{C}\right]-\mathrm{HECADE}$ \\
\hline
\end{tabular}

Figure 4.4: Newman projections of the conformers 9 and 11 for dihedral C12-C21. All conformers have a configuration of $(12 R, 21 R)$. Small negative coupling constants observed for both $\mathrm{H} 21 / \mathrm{C} 12$ and $\mathrm{H} 21 / \mathrm{C} 11$, together with a small couplings of $\mathrm{H} 12 / \mathrm{C} 22$, suggest conformer 11 as the main conformation.

\subsubsection{Elucidation of the absolute configuration by chiroptics}

To determine the absolute configuration, ECD spectra of both enantiomeric forms $(12 S, 21 S)$ and $(12 R, 21 R)$ of NMR-determined conformation 11 have been calculated with time-dependent density functional theory (TD-DFT). The ECD calculations were conducted with the B3LYP/6-31G(d) basis set using the IEFPCM solvent continuum model with DMSO as the solvent. The ECD spectrum calculated for $(12 R, 21 R)$ shown in Figure 4.7a reproduced both the signs and the shape of the measured one. The agreement between the recorded spectrum and the calculated one is excellent, when the calculated spectrum of $(12 R, 21 R)$ is red-shifted by $25 \mathrm{~nm}$, 


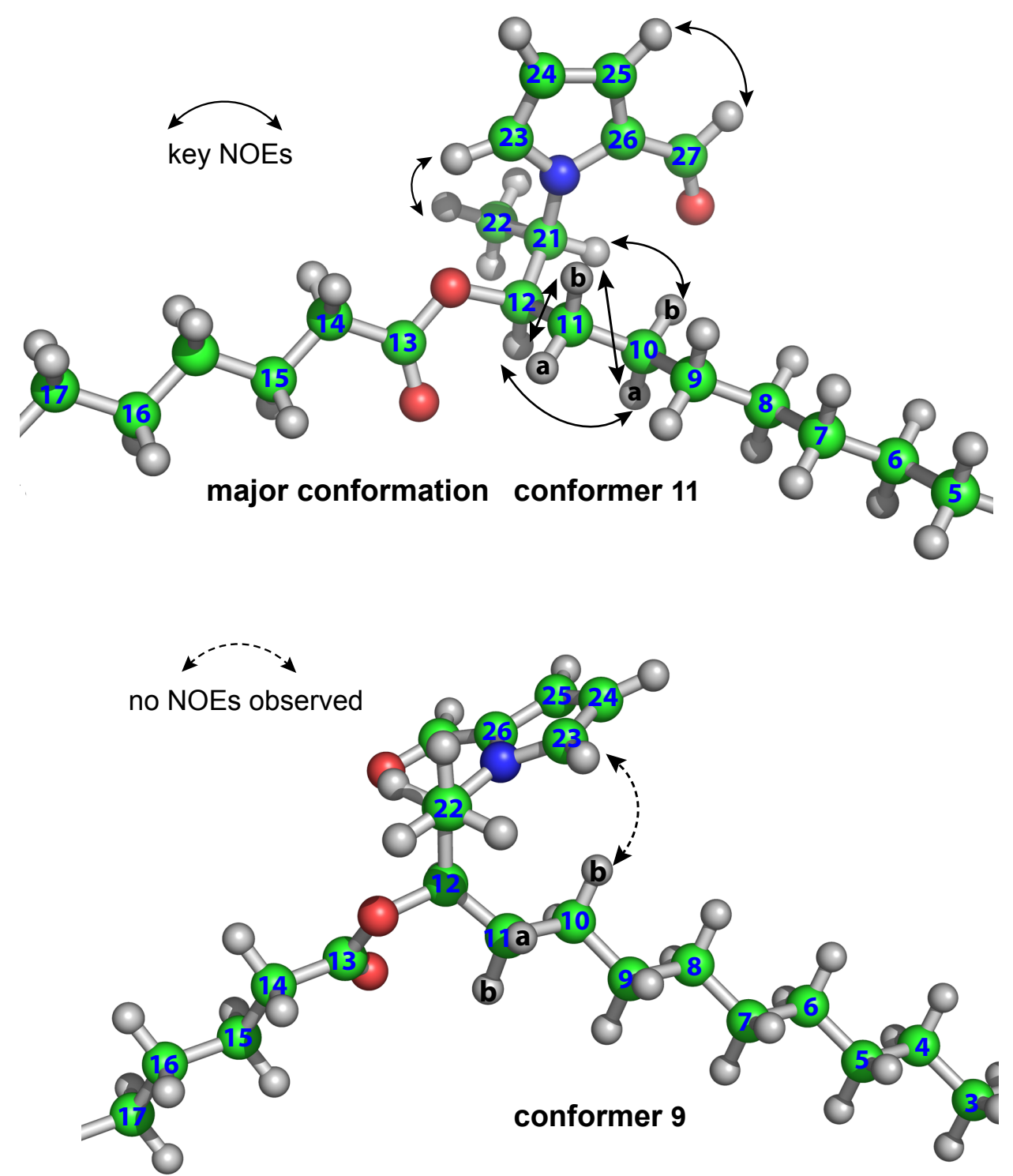

Figure 4.5: Key NOE correlations additionally supporting the NMR-determined conformer 11 and relative configuration $(12 S, 21 S)$ or $(12 R, 21 R)$ of LLG1 and the absence of the NOE in the NOESY spectrum excluding conformer 9 as the major conformation.

(Figure 4.7b). This procedure is in general allowed, because it has been shown in many previous studies that using the TD-DFT calculation with the B3LYP functionals the excitation energy can be predicted either too high or too low, depending on which system class is taken into account [Diedrich and Grimme, 2003]. Based on the ECD data, the absolute configuration of LLG1 was assigned as $(12 R, 21 R)$.

Furthermore we measured ORD values at five different wavelengths $(365,436$, 546, 578, $589 \mathrm{~nm}$ ) in DMSO for LLG1, and calculated them for the conformer 

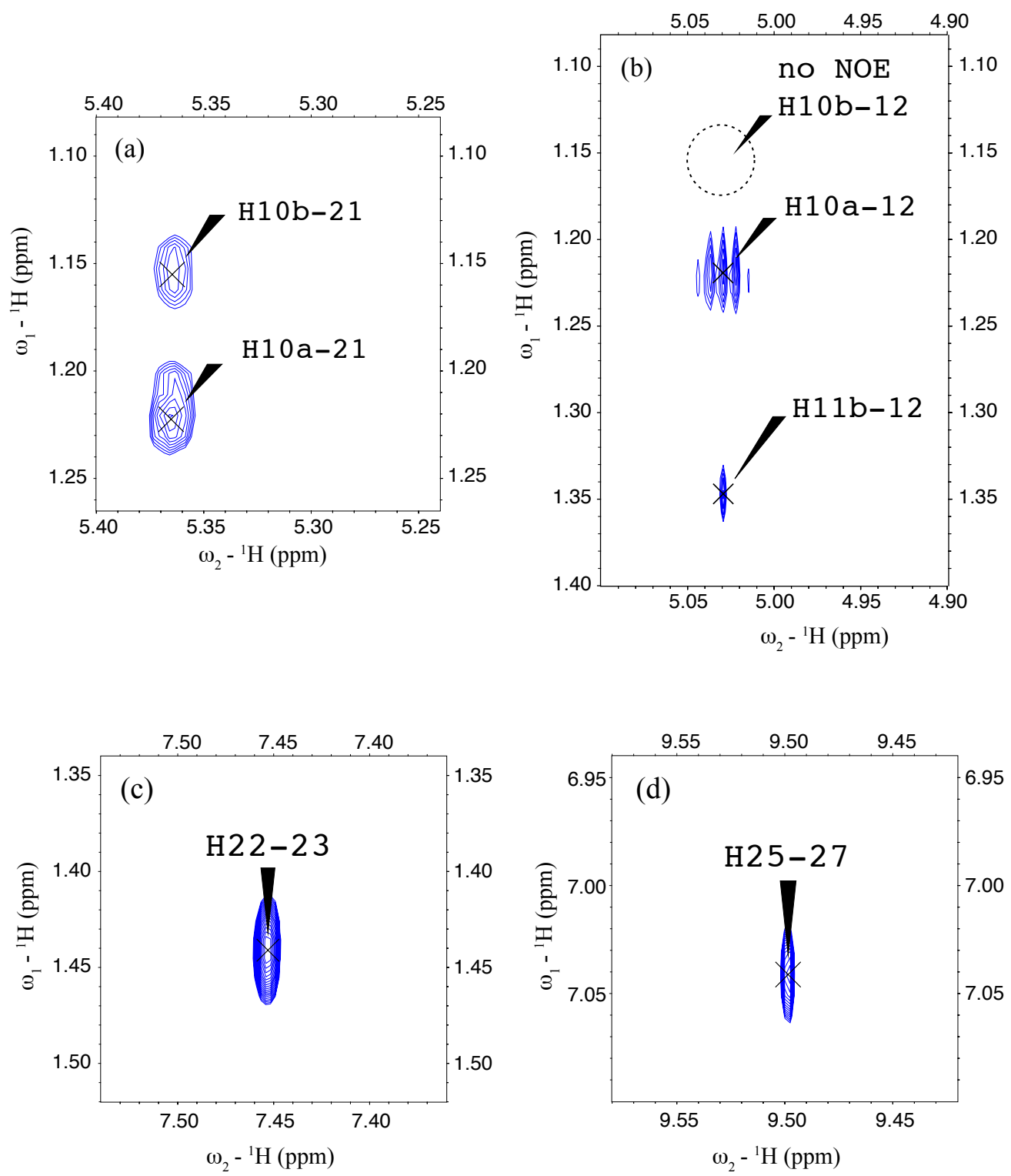

Figure 4.6: Selected regions of NOESY spectrum of LLG1. The mixing time of the NOESY spectrum is $200 \mathrm{~ms}$.

11 with DFT at the B3LYP/6-31G(d) level using the IEFPCM solvent continuum model with DMSO as the solvent. Figure 4.8 shows that the calculated ORD curve of $(12 R, 21 R)$ has a very good agreement with the experimental one, which further approved the assignment of the absolute configuration from the ECD data. In conclusion, comparison of the DFT computations of the NMR-determined conformation with the experimental data shows that both ECD and ORD yield the same absolute configuration determination for LLG1, which is $(12 R, 21 R)$. 
Table 4.4: Comparison of the NOE integrals and the interproton distances for the best RDC-fitting conformer 11. The NOESY spectrum was acquired with a mixing time of $200 \mathrm{~ms}$. The distance to the power of minus six is referenced to proton pair H10b-H21.

\begin{tabular}{llll}
\hline Atoms & NOE integrals & $\begin{array}{c}\text { Distance } \\
(\AA)\end{array}$ & $\begin{array}{c}\text { (Distance) }^{-6} \\
\text { (Referenced to H10b-H21) }\end{array}$ \\
\hline H12-H10a & $5.60 \times 10^{5}$ & 2.53 & 0.56 \\
H22-H23 & $7.15 \times 10^{5}$ & 2.61 & 0.47 \\
H25-H27 & $2.10 \times 10^{5}$ & 2.55 & 0.54 \\
H10b-H21 & $1.11 \times 10^{5}$ & 2.30 & 1.00 \\
H10a-H21 & $1.40 \times 10^{5}$ & 3.11 & 0.16 \\
H11b-H12 & $1.81 \times 10^{5}$ & 3.06 & 0.18 \\
H11a-H12 & $4.97 \times 10^{5}$ & 2.50 & 0.61 \\
H21-H27 & $3.20 \times 10^{4}$ & 3.73 & 0.05 \\
\hline
\end{tabular}

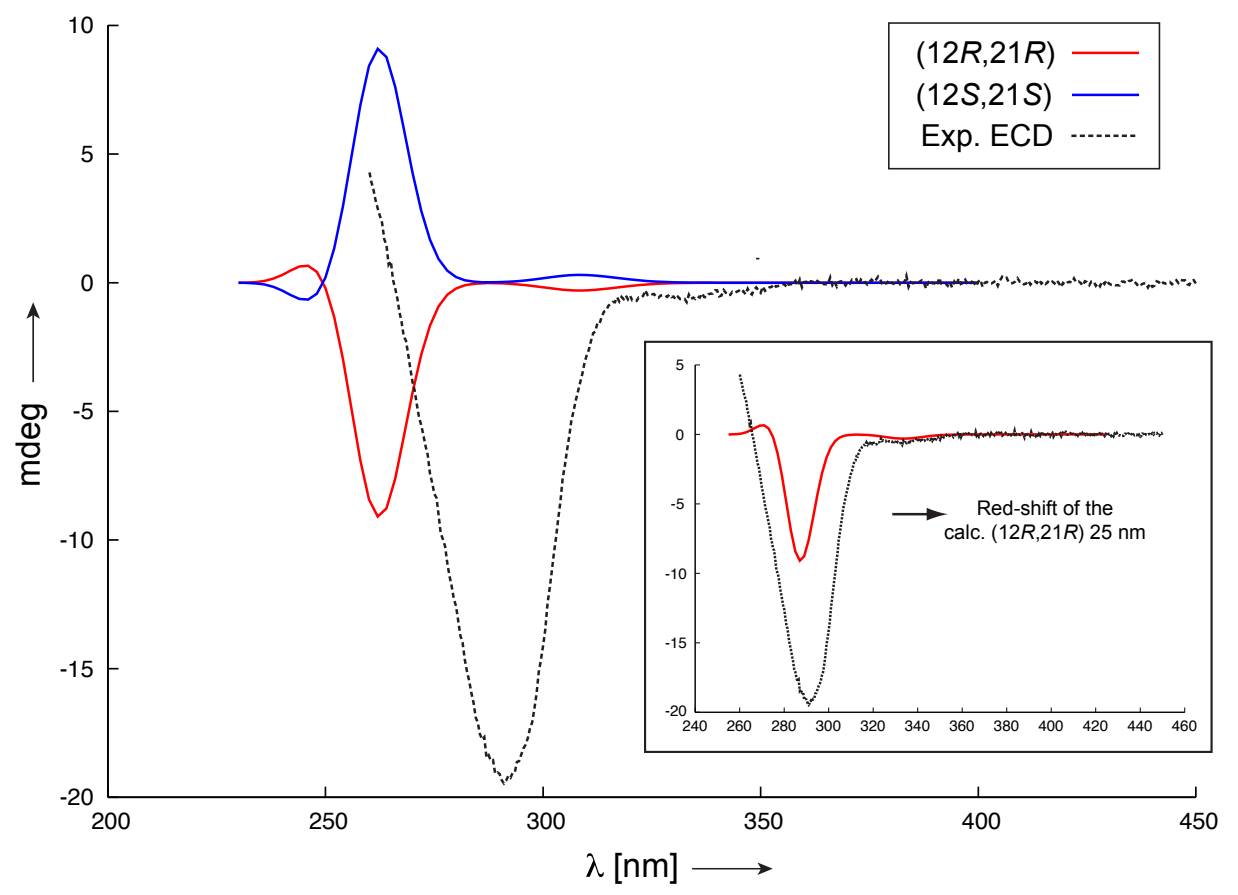

Figure 4.7: (a) Comparison of experimentally measured ECD spectrum of LLG1 in DMSO (dashed line) to the calculated ones using the RDC determined conformer 11 for both enantiomers $(12 S, 21 S)$ and $(12 R, 21 R)$. (b) A $25 \mathrm{~nm}$ shift to higher wavelength of the calculated ECD spectrum of $(12 R, 21 R)$ results in a good fit to the experimental spectrum. 


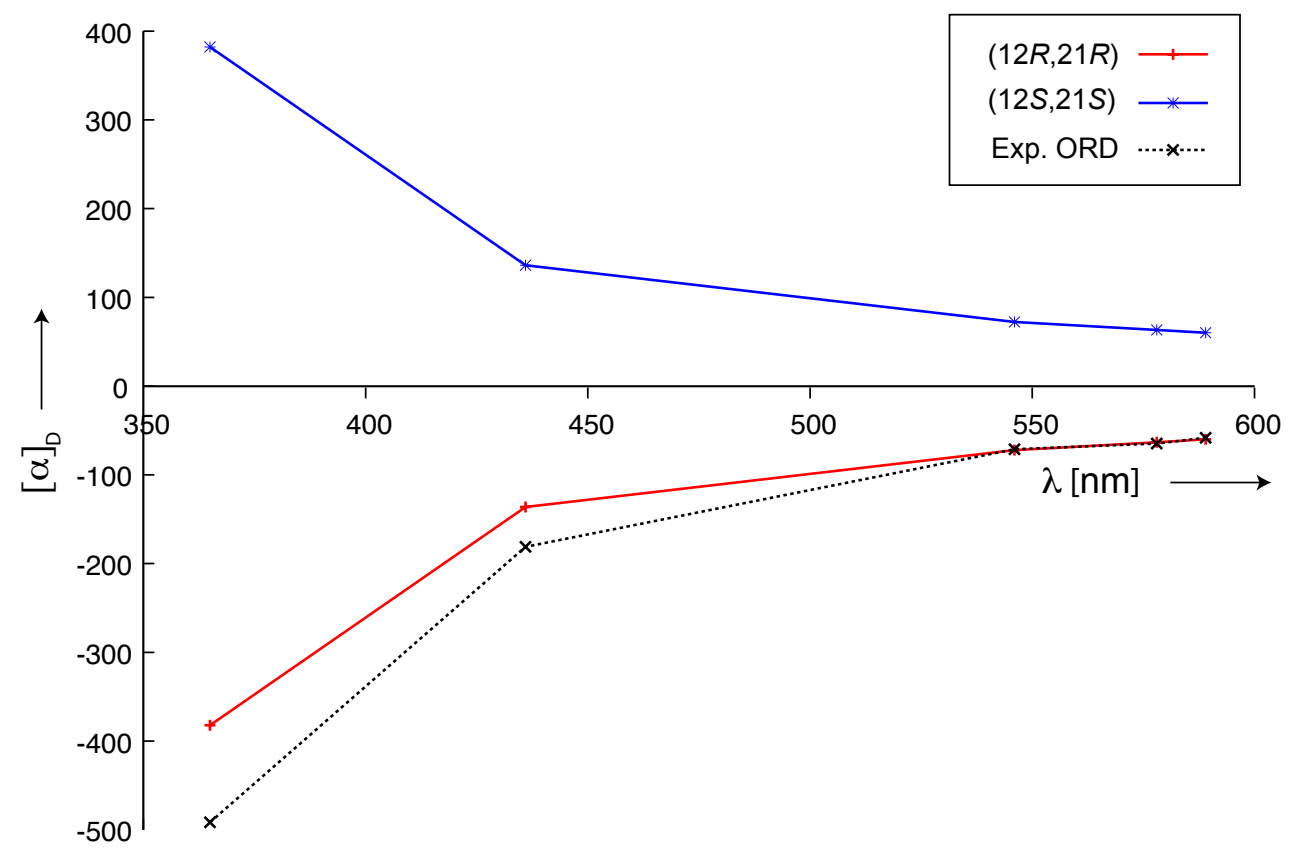

Figure 4.8: Comparison of experimentally measured ORD spectrum of LLG1 in DMSO (dashed line) to the calculated ones using the RDC determined conformer 11 for both enantiomers $(12 S, 21 S)$ and $(12 R, 21 R) .1 \mathrm{mg}$ of LLG1 was used for the ORD measurements. The accuracy of the mass measurement is $1 \pm 0.3 \mathrm{mg}$.

\subsubsection{Conclusion}

The absolute configuration of LLG1 has been established as $(12 R, 21 R)$ using a combination of NMR and computationally supported chiroptical spectroscopy. It is worth to note that in this study without the aid of the RDC analysis, an unambiguous determination of configuration and conformation was not feasible due to the excessive conformational possibilities of this open-chain compound. Using the SVD fitting of the ${ }^{1} D_{\mathrm{CH}}$ data on the individual possible conformers, not only the relative configuration was successfully established, but also the selection of the correct conformations has been remarkably simplified. A NOE and $J$-coupling analysis was subsequently utilized to validate the RDC-determined conformation and configuration. To our surprise, disregarding the potential conformational flexibility in the alkyl chains, prevalence of one conformer around the dihedral of C11-C12, C12-C21 and $\mathrm{C} 21-\mathrm{N}$ has been determined in solution, which is shown in Figure 4.9. In this figure, the alkyl chains $\mathrm{C} 1-\mathrm{C} 10$ and $\mathrm{C} 14-\mathrm{C} 20$ were adopted to have an anti conformation, which should be the lowest energy conformer [Nasipuri, 2012]. According to the literature [Hoffmann, 2000], it is interesting to note that high conformational preferences have been already observed for numerous open-chain polyketides, especially when the alkyl chains contain certain functional groups. For example the 
methyl groups in the alkyl chains do not affect the flexibility of the backbone, nevertheless they reduce the number of low energy local conformers. Our study here shows that LLG1 is another case, in which nature has chosen an acyclic backbone with a high preference to populate a preferred local conformation.

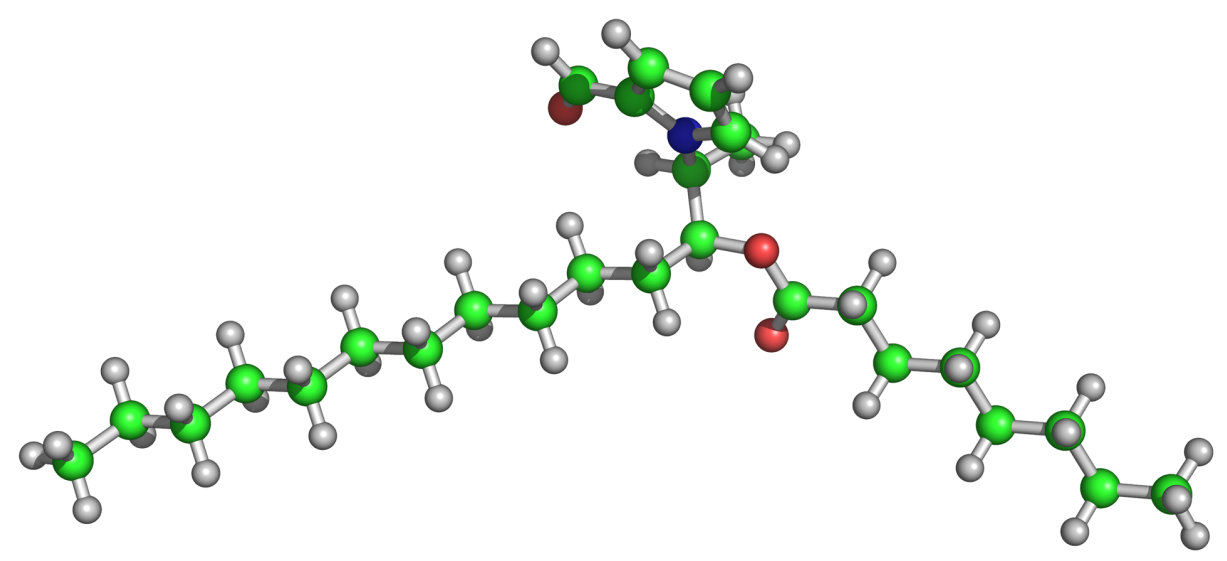

Figure 4.9: Major conformation of LLG1 with the determined absolute configuration $(12 R, 21 R)$.

\subsection{Determination of the absolute configuration of vatiparol}

\subsubsection{Introduction}

Vatiparol is a new identified resveratrol trimer from Vatica parvifolia. Resveratrol is a natural polyphenol that exists as a phytoalexin in grapes and other plant-derived foodstuffs, and has been shown to display an array of biological activities, such as cancer chemoprevention [Jang et al., 1997], lifespan extension [Baur et al., 2006], and selective disruption of amyloid fibrils, which are associated with the incidence of Alzheimer's disease [Ladiwala et al., 2010]. Using it as a starting unit, the plant species of at least nine families - including Dipterocarpaceae, Cyperaceae, Gnetaceae, Iridaceae, Leguminosae, Moraceae, Orchidaceae, Polygonaceae and Paeoniaceae [Shen et al., 2009, Choi et al., 2011] (though possibly all families may use this) - can biochemically synthesize a diversity of resveratrol oligomers. Some of these have antitumor activity [Lee et al., 2006], are antioxidant [He et al., 2008], activate PPARs (peroxisome proliferator-activated receptors) [Tsukamoto et al., 2010], inhibit the $\alpha$-secretase BACE-15, and are anti-inflammatory [Chung et al., 2003]. Furthermore, some irregular resveratrol oligomers, such as immunosuppressor dip- 
toindonesin G [Ge et al., 2010] and acetylcholinesterase inhibitor hopeahainol A [Ge et al., 2008] have been characterized from Hopea chinensis and Hopea hainanensis (Dipterocarpaceae).

As a rich source of resveratrol oligomers the Vatica genus (Dipterocarpaceae), consisting of over sixty small-to-medium sized trees growing mainly in Southeast Asia, has been identified. It is an efficient producer of uniquely structured resveratrol oligomers possessing diverse biological activities [Ladiwala et al., 2010]. However, Vatica parvifolia remains poorly investigated, presumably because it is a rare species endemic to the Hainan Island of China. After careful isolation and characterization, vatiparol (Figure 4.10), a minor resveratrol trimer with a novel carbon skeleton has been discovered by our collaborators Dr. Ming-Hui Ge and Prof. Ren-Xiang Tan from Institute of Functional Biomolecules, School of Lifesciences, Nanjing University, Nanjing, China.

Assignment of the configuration of vatiparol was complicated since it has neither acylable hydroxyl groups, which precludes the commonly used Mosher's approach, nor a well recognizable chirality-associated chromophore, which excludes the exciton chirality method. Moreover, the molecule could also not be crystallized by using single or composite solvents according to our collaborators. Therefore the goal of this study was to determine the absolute configuration of vatiparol using our established method of combining RDC enhanced NMR and DFT-supported chiroptical spectroscopy. Furthermore, since only trace amounts of vatiparol have been isolated, a new technique for the establishment of the PH-gels in $1.7 \mathrm{~mm}$ NMR tubes for the RDC measurements was introduced.

\subsubsection{Elucidation of the relative configuration and conforma- tion by NMR}

\section{Determination of the constitution and NMR resonance assignment}

Vatiparol, a pale yellow powder, gave an $[\mathrm{M}+\mathrm{Na}]^{+}$ion at $m / z 701.1779\left(\left[\mathrm{C}_{42} \mathrm{H}_{30} \mathrm{O}_{9} \mathrm{Na}\right]^{+}\right.$, calcd 701.1782) in its high resolution electrospray-ionization mass spectrum; this suggests that it is most likely a resveratrol trimer following correlation of its molecular formula with that of resveratrol. However, the ${ }^{1} \mathrm{H}-\mathrm{NMR}$ and ${ }^{1} \mathrm{H},{ }^{1} \mathrm{H}-\mathrm{COSY}$ spectra exhibited only two 1,2,3,5-tetrasubstituted benzene rings ("heads" of resveratrol units), which resonated as two pairs of doublets at $\delta\left({ }^{1} \mathrm{H}\right)=6.68$ (H10a) and $6.01 \mathrm{ppm}(\mathrm{H} 12 \mathrm{a})(J=2.1 \mathrm{~Hz})$ as well as at $\delta\left({ }^{1} \mathrm{H}\right)=6.21(\mathrm{H} 10 \mathrm{c})$ and $6.45 \mathrm{ppm}$ 


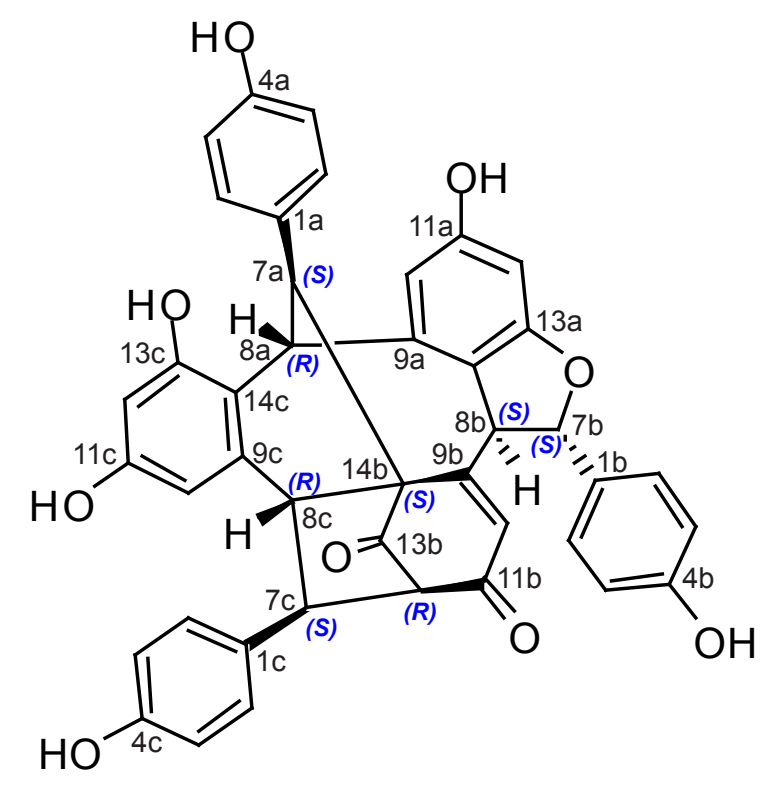

Figure 4.10: The structure of vatiparol with atom numbering and determined absolute configuration.

(H12c) $(J=1.7 \mathrm{~Hz})$. In addition to having an HSQC cross-peak with the carbon signal at $\delta\left({ }^{13} \mathrm{C}\right)=125.5 \mathrm{ppm}$, the olefinic singlet at $\delta\left({ }^{1} \mathrm{H}\right)=5.85 \mathrm{ppm}$ showed HMBC correlations to the quaternary carbon appearing at $\delta\left({ }^{13} \mathrm{C}\right)=61.4 \mathrm{ppm}$, and to the methine carbon signal at $\delta\left({ }^{13} \mathrm{C}\right)=70.7 \mathrm{ppm}$. Rationalizing the magnitude of the three non-protonated carbon resonance lines at $\delta\left({ }^{13} \mathrm{C}\right)=204.2,193.9$ and $168.9 \mathrm{ppm}$, the spectral evidence highlighted that one of the 3,5-dihydroxyphenyl residues was transformed into a 2,5,6,6-tetrasubstituted cyclohex-4-ene-1,3-dione moiety during the resveratrol oligomerization in the plant.

The molecular formula suggested that four additional non-aromatic rings had to be proposed for the structure of vatiparol. Further interpretation of the ${ }^{1} \mathrm{H}$ NMR and ${ }^{1} \mathrm{H},{ }^{1} \mathrm{H}$-COSY spectra disclosed that a sequence of three vicinally coupled methine protons resonating at $\delta\left({ }^{1} \mathrm{H}\right)=3.82(\mathrm{~d}, J=7.5 \mathrm{~Hz}, \mathrm{H} 12 \mathrm{~b}), 3.98(\mathrm{t}, J=7.5 \mathrm{~Hz}$, $\mathrm{H} 7 \mathrm{c})$ and $3.45 \mathrm{ppm}(\mathrm{d}, J=7.5 \mathrm{~Hz}, \mathrm{H} 8 \mathrm{c})$ was accompanied by two pairs of mutually coupled doublets at $\delta\left({ }^{1} \mathrm{H}\right)=3.59(\mathrm{H} 7 \mathrm{a})$ and $5.65 \mathrm{ppm}(\mathrm{H} 8 \mathrm{a})(J=2.8 \mathrm{~Hz})$ as well as at $\delta\left({ }^{1} \mathrm{H}\right)=5.62(\mathrm{H} 7 \mathrm{~b})$ and $3.85 \mathrm{ppm}(\mathrm{H} 8 \mathrm{~b})(J=10.3 \mathrm{~Hz})$. Subsequently, the HMBC spectrum of vatiparol was found to map unambiguously these sequences of coupled nuclei with the aforementioned substructures. Furthermore a 2-(4-hydroxyphenyl)2,3-dihydrofuran motif was required by the key HMBC correlations of H7b with $\mathrm{C} 2 \mathrm{~b} / 6 \mathrm{~b}, \mathrm{C} 9 \mathrm{~b}$ and C14a, and of H8b with C1b, C9a, C13a and C10b. Moreover, a 
five-membered carbocycle with C7c carrying a 4-hydroxyphenyl group was demonstrated to be fused to the cyclohex-4-ene-1,3-dione moiety by the HMBC correlations of $\mathrm{H} 7 \mathrm{c}$ with $\mathrm{C} 2 \mathrm{c} / 6 \mathrm{c}$ and $\mathrm{C} 11 \mathrm{~b}$, of $\mathrm{H} 8 \mathrm{c}$ with $\mathrm{C} 10 \mathrm{c}, \mathrm{C} 13 \mathrm{~b}$ and $\mathrm{C} 14 \mathrm{c}$, and of $\mathrm{H} 12 \mathrm{~b}$ with $\mathrm{C} 10 \mathrm{~b}$ and $\mathrm{C} 14 \mathrm{~b}$. As suggested by the biogenetic consideration, the six- and sevenmembered rings were constructed by connecting C7a with C1a, C8a and C14b. This hypothesis was substantiated by HMBC correlations of $\mathrm{H} 7 \mathrm{a}$ with $\mathrm{C} 2 \mathrm{a} / 6 \mathrm{a}, \mathrm{C} 9 \mathrm{a}, \mathrm{C} 9 \mathrm{~b}$, $\mathrm{C} 13 \mathrm{~b}$ and $\mathrm{C} 14 \mathrm{c}$, and of $\mathrm{H} 8 \mathrm{a}$ with $\mathrm{C} 1 \mathrm{a}, \mathrm{C} 10 \mathrm{a}, \mathrm{C} 14 \mathrm{~b}, \mathrm{C} 9 \mathrm{c}$ and $\mathrm{C} 13 \mathrm{c}$. Thus, the constitution of vatiparol with a carbon skeleton that had previously never been found was determined, and it was further corroborated by the excellent fitting of the RDC data, as shown below. The ${ }^{1} \mathrm{H}$ and ${ }^{13} \mathrm{C}$ chemical shifts of vatiparol are listed in Table 4.5 .

\section{Measurements of scalar and residual dipolar couplings}

${ }^{3} J_{\mathrm{HH}}$ couplings were extracted from an $1 \mathrm{D}-{ }^{1} \mathrm{H}$ spectrum, and long-range proton carbon couplings were derived with $\left[{ }^{1} \mathrm{H}_{-}{ }^{13} \mathrm{C}\right]-\mathrm{HECADE}$ and $\left[{ }^{1} \mathrm{H}_{-}{ }^{13} \mathrm{C}\right]-\mathrm{HSQMBC}$ experiments. All couplings are listed in Table 4.6.

As vatiparol was isolated from a very rare species of the plant, the limited availability of the sample constitutes a methodological challenge for the RDC measurements using the conventionally applied methods. To overcome the present limitations, we introduced a preparation of a slim PH-gel for $1.7 \mathrm{~mm}$ NMR tubes for commercial high-resolution NMR probe heads for the first time. In this slim-gel procedure, we used the same concentration of the stock solution as for regular $\mathrm{PH}-$ gels [Haberz et al., 2005] and filled it in a $2.0 \mathrm{~mm}$ cylinder instead of a $5.4 \mathrm{~mm}$ cylinder, which is used for $5 \mathrm{~mm}$ NMR tubes. The comparison of the apparatus for preparing the gels with different sizes is shown in the materials and methods chapter (Figure 3.1). The polymerization was accomplished in $8-9$ min at $70{ }^{\circ} \mathrm{C}$. Longer polymerization times made the gels very fragile. After being washed and dried, the gel can be easily introduced into a $1.7 \mathrm{~mm}$ NMR tube together with DMSO and the sample. A full re-swelling of the gel took 2-3 weeks, similar to gels in $5 \mathrm{~mm}$ tubes. In our study, a deuterium quadrupolar splitting of $10.3 \mathrm{~Hz}$ was observed and 16 ${ }^{1} D_{\mathrm{CH}}$ values in the range of -45 to $50 \mathrm{~Hz}$ could be extracted for vatiparol by means of $\left[{ }^{1} \mathrm{H},{ }^{13} \mathrm{C}\right]$-CLIP-HSQC experiments. In addition to the sensitivity advantage of a factor of 5 due to the usage of a $1.7 \mathrm{~mm}$ instead of $5 \mathrm{~mm}$ NMR tube when using the same amount of sample, we noted much sharper resonance lines than for anisotropic samples prepared in $5 \mathrm{~mm}$ tubes. This might be due to easier shimming in the tube with the smaller diameter, but could also arise from the more homogeneous gel 
Table 4.5: Carbon and proton chemical shifts of vatiparol in DMSO- $d_{6}$ at $298 \mathrm{~K}$.

\begin{tabular}{|c|c|c|c|}
\hline Carbon & Chemical shift (ppm) & Proton & Chemical shift (ppm) \\
\hline C1a & 130.6 & & \\
\hline $\mathrm{C} 2 \mathrm{a}, 6 \mathrm{a}$ & 128.5 & $\mathrm{H} 2 \mathrm{a}, 6 \mathrm{a}$ & 7.05 \\
\hline C3a, $5 \mathrm{a}$ & 115.8 & H3a, $5 \mathrm{a}$ & 6.72 \\
\hline $\mathrm{C} 4 \mathrm{a}$ & 156.4 & & \\
\hline $\mathrm{C} 7 \mathrm{a}$ & 39.3 & $\mathrm{H} 7 \mathrm{a}$ & 3.59 \\
\hline $\mathrm{C} 8 \mathrm{a}$ & 37.3 & $\mathrm{H} 8 \mathrm{a}$ & 5.47 \\
\hline C9a & 140.0 & & \\
\hline C10a & 109.1 & $\mathrm{H} 10 \mathrm{a}$ & 6.68 \\
\hline C11a & 158.9 & & \\
\hline C12a & 95.8 & $\mathrm{H} 12 \mathrm{a}$ & 6.01 \\
\hline C13a & 158.9 & & \\
\hline C14a & 114.5 & & \\
\hline C1b & 128.8 & & \\
\hline $\mathrm{C} 2 \mathrm{~b}, 6 \mathrm{~b}$ & 129.3 & $\mathrm{H} 2 \mathrm{~b}, 6 \mathrm{~b}$ & 6.91 \\
\hline $\mathrm{C} 3 \mathrm{~b}, 5 \mathrm{~b}$ & 115.7 & $\mathrm{H} 3 \mathrm{~b}, 5 \mathrm{~b}$ & 6.71 \\
\hline $\mathrm{C} 4 \mathrm{~b}$ & 158.4 & & \\
\hline $\mathrm{C} 7 \mathrm{~b}$ & 86.4 & $\mathrm{H} 7 \mathrm{~b}$ & 5.62 \\
\hline $\mathrm{C} 8 \mathrm{~b}$ & 51.0 & $\mathrm{H} 8 \mathrm{~b}$ & 3.85 \\
\hline $\mathrm{C} 9 \mathrm{~b}$ & 168.9 & & \\
\hline $\mathrm{C} 10 \mathrm{~b}$ & 125.5 & $\mathrm{H} 10 \mathrm{~b}$ & 5.85 \\
\hline C11b & 193.9 & & \\
\hline $\mathrm{C} 12 \mathrm{~b}$ & 70.7 & $\mathrm{H} 12 \mathrm{~b}$ & 3.82 \\
\hline $\mathrm{C} 13 \mathrm{~b}$ & 204.2 & & \\
\hline $\mathrm{C} 14 \mathrm{~b}$ & 61.4 & & \\
\hline $\mathrm{C} 1 \mathrm{c}$ & 128.9 & & \\
\hline $\mathrm{C} 2 \mathrm{c}, 6 \mathrm{c}$ & 129.6 & $\mathrm{H} 2 \mathrm{c}, 6 \mathrm{c}$ & 7.07 \\
\hline $\mathrm{C} 3 \mathrm{c}, 5 \mathrm{c}$ & 116.1 & $\mathrm{H} 3 \mathrm{c}, 5 \mathrm{c}$ & 6.74 \\
\hline $\mathrm{C} 4 \mathrm{c}$ & 157.0 & & \\
\hline $\mathrm{C} 7 \mathrm{c}$ & 45.1 & H7c & 3.98 \\
\hline $\mathrm{C} 8 \mathrm{c}$ & 50.2 & $\mathrm{H} 8 \mathrm{c}$ & 3.45 \\
\hline $\mathrm{C} 9 \mathrm{c}$ & 137.4 & & \\
\hline C10c & 104.6 & $\mathrm{H} 10 \mathrm{c}$ & 6.01 \\
\hline $\mathrm{C} 11 \mathrm{c}$ & 157.0 & & \\
\hline $\mathrm{C} 12 \mathrm{c}$ & 101.8 & $\mathrm{H} 12 \mathrm{c}$ & 6.36 \\
\hline $\mathrm{C} 13 \mathrm{c}$ & 153.6 & & \\
\hline $\mathrm{C} 14 \mathrm{c}$ & 121.4 & & \\
\hline
\end{tabular}


Table 4.6: Long-range proton-proton and proton-carbon coupling constants of vatiparol in DMSO.

\begin{tabular}{lcll}
\hline Atoms & ${ }^{3} J_{\mathrm{HH}}(\mathrm{Hz})$ & Atoms & ${ }^{3} J_{\mathrm{CH}}(\mathrm{Hz})$ \\
\hline H7a-H8a & 2.8 & H7a-C9a & 8.2 \\
H7b-H8b & 10.3 & H7a-C14c & 3.0 \\
H7c-H8c & 7.5 & H8a-C1a & 2.8 \\
H7c-H12b & 7.5 & H8a-C14b & 8.0 \\
& & H8b-C7b & -5.6 \\
& & H8b-C10b & 3.1 \\
& & H12b-C10b & 2.5 \\
& & H7c-C12b & -5.0 \\
& & H8c-C10c & 1.5 \\
\hline
\end{tabular}

Table 4.7: Residual dipolar couplings of vatiparol in PH-gel/DMSO.

\begin{tabular}{lclc}
\hline CH-vector & ${ }^{1} D_{\mathrm{CH}}(\mathrm{Hz})$ & CH-vector & ${ }^{1} D_{\mathrm{CH}}(\mathrm{Hz})$ \\
\hline C2a,6a-H2a,6a & -3.7 & C10b-H10b & 39.6 \\
C7a-H7a & 17.5 & C12b-H12b & -13.8 \\
C8a-H8a & -4.2 & C2c,6c-H2c,6c & -2.0 \\
C10a-H10a & 50.3 & C3c,5c-H3c,5c & -4.4 \\
C12a-H12a & 4.5 & C7c-H7c & 20.1 \\
C2b,6b-H2b,6b & -3.4 & C8c-H8c & 5.8 \\
C7b-H7b & -44.1 & C10c-H10c & -30.6 \\
C8b-H8b & -40.9 & C12c-H12c & 51.5 \\
\hline
\end{tabular}

in the smaller volume. A section of the overlaid isotropic and anisotropic spectra measured using a slim-gel in a $1.7 \mathrm{~mm}$ NMR tube is shown in Figure 4.11.

\section{Configurational analysis}

Starting from the constitution of vatiparol elucidated above, we tried to establish the relative and absolute configurations of the eight unknown stereocenters $\mathrm{C} 7 \mathrm{a}$, C8a, C7b, C8b, C12b, C14b, C7c and C8c. The J-coupling analysis shown in Figure A.4 enables to establish the relative configuration of $\mathrm{C} 7 \mathrm{a}$ and $\mathrm{C} 8 \mathrm{a}$ to be syn $(R S$ or $S R)$. The configuration of $\mathrm{C} 7 \mathrm{~b}$ and $\mathrm{C} 8 \mathrm{~b}$ was determined as anti $(R R$ or $S S$ ) due to the large coupling constants of $\mathrm{H} 7 \mathrm{~b} / \mathrm{H} 8 \mathrm{~b}$ (Table 4.6) and a relatively small NOE between this proton pair (Table 4.8). Based on geometric considerations 


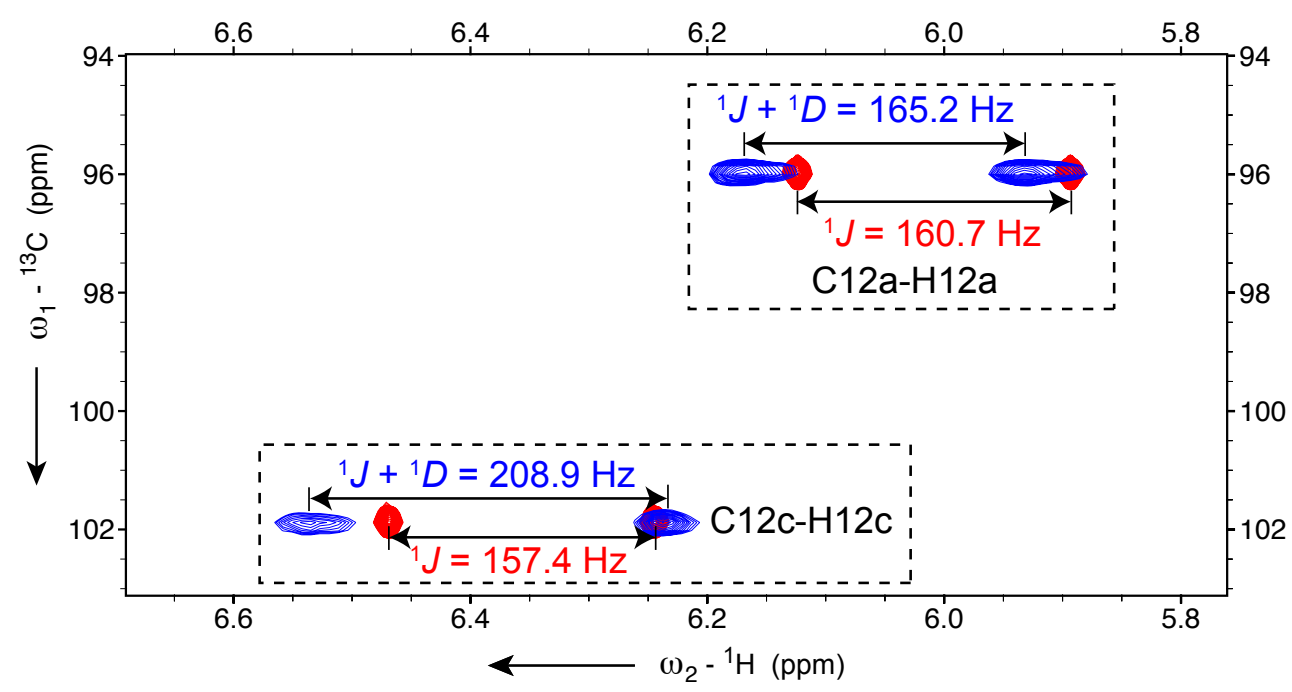

Figure 4.11: A section of overlaid isotropic and anisotropic spectra measured using a slimgel in a $1.7 \mathrm{~mm}$ NMR tube. ${ }^{1} D_{\mathrm{CH}}$ values are extracted as the difference of the splitting between isotropic and anisotropic signals.

the relative configuration of $\mathrm{C} 7 \mathrm{a} / \mathrm{C} 14 \mathrm{~b}$ should be anti and $\mathrm{C} 12 \mathrm{~b} / \mathrm{C} 14 \mathrm{~b}$ syn. Using these restraints, the 128 possible configurations of vatiparol can be reduced to 16 , which are $R S R R S R S S, R S R R S R R S, R S R R S R R R, R S S S S R R S, R S R R S R S R$, RSSSSRRR, RSSSSRSS and RSSSSRSR for C7a, C8a, C7b, C8b, C12b, C14b, $\mathrm{C} 7 \mathrm{c}$ and $\mathrm{C} 8 \mathrm{c}$, respectively, and their enantiomers. We, therefore, manually built one structure per diastereomer in Discovery Studio 2.5 and energy-minimized these using the CFF force field [Lifson and Warshel, 1968]. The structural models were further optimized with DFT at the B3LYP/3-21G(d) and B3LYP/6-31G(d) level using the IEFPCM solvent continuum model with DMSO as the solvent. The conformations did not change significantly when a further optimization with the larger basis set B3LYP/6-311G(2d,p) was performed.

To evaluate the possible diastereomers we tested the optimized conformation of each configuration against the NOE data. Quantitative NOE build-up curves for five different mixing times varying from 200 to $400 \mathrm{~ms}$ were derived from NOESY experiments with the suppression of zero-quantum interference [Thrippleton and Keeler, 2003]. Altogether 56 NOE distances (Table 4.8) were integrated at $300 \mathrm{~ms}$ mixing time and translated into proton-proton distances, which were referenced to $\mathrm{H} 10 \mathrm{a} / \mathrm{H} 12 \mathrm{a}$, considering the distance between two meta proton pairs in an aromatic ring to be $4.29 \AA$. These NOEs include 41 long-range NOEs defined here as NOEs between two protons separated by more than four bonds. The 8 possible configurations have been evaluated based on the calculation of the total NOE violations of each 
optimized structure. Figure 4.12 shows clearly that the configuration $S R S S R S S R$ (or its enantiomer) has the lowest total NOE violations out of the eight possible diastereomers. Furthermore, no significant individual NOE violation can be found for this configuration (Table A.4).

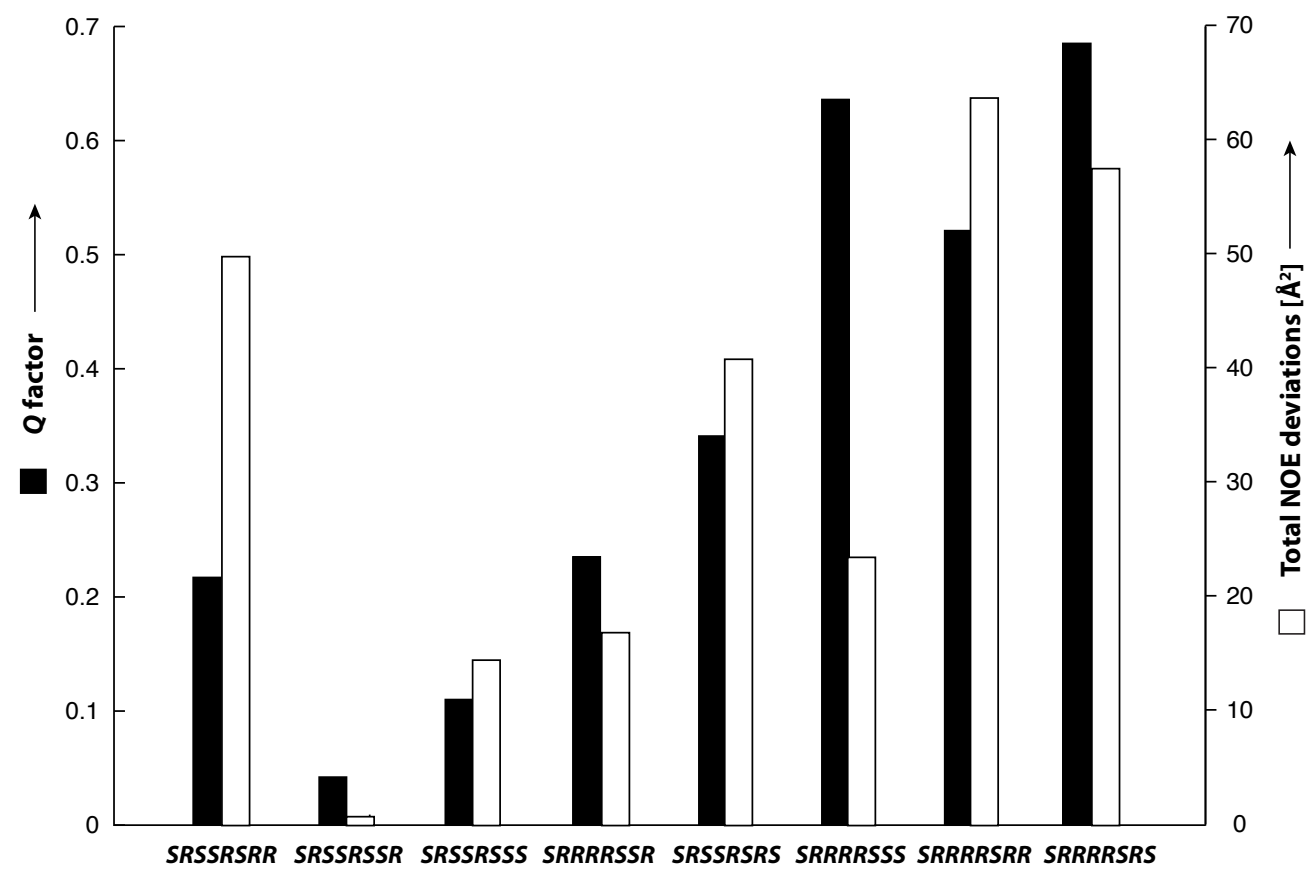

Figure 4.12: Comparison of $Q$ factors of the RDC data and total NOE deviations of 56 NOEs for eight possible configurations of vatiparol. The structural models for the NOE and RDC analysis were optimized with DFT at at the B3LYP/6-31G(d) level using the IEFPCM solvent continuum model with DMSO as the solvent. The fitting of thirteen ${ }^{1} D_{\mathrm{CH}}$ values from the core structure was performed with the SVD method. The 56 NOEs were derived from a NOESY experiment with 300 ms mixing time.

Although the NOEs are very discriminative for relative configuration, we measured RDCs of vatiparol to independently validate the relative configuration, and establish the conformation of the rotatable phenyl rings. The latter is necessary for the accurate calculation of the chiroptical properties of the molecule. The determination of the torsion angles of the three potentially freely rotating phenyl rings is very difficult from NOEs between protons in the ring and on the carbon to which the phenyl ring is attached due to the weak distance dependence on the torsion angle.

For evaluating different diastereomers independent of the NOE analysis, $12{ }^{1} D_{\mathrm{CH}}$ values from the core structure, excluding RDCs from the potentially rotatable phenyl rings, were analyzed by using singular value decomposition (SVD). Consistent with 
Table 4.8: Proton-proton distances of vatiparol derived from NOESY spectra at $300 \mathrm{~ms}$ mixing time. All distances were referenced to H10a/H12a, considering the distance between two meta proton pairs in an aromatic ring to be $4.29 \AA$.

\begin{tabular}{llll}
\hline Atoms & Distance $(\AA)$ & Atoms & Distance $(\AA)$ \\
\hline H2a,6a-H7a & 3.0 & H3b,5b-H7b & 4.1 \\
H2a,6a-H8a & 2.4 & H3b,5b-H10b & 4.7 \\
H2a,6a-H10a & 3.5 & H7b-H8b & 3.2 \\
H2a,6a-H2,6b & 4.2 & H7b-H10b & 2.5 \\
H2a,6a-H8b & 3.2 & H7b-H2c,6c & 3.5 \\
H2a,6a-H10b & 4.8 & H7b-H3c,5c & 5.0 \\
H3a,5a-H7a & 4.3 & H7b-H8c & 3.7 \\
H3a,5a-H8a & 3.8 & H8b-H10b & 3.6 \\
H3a,5a-H8b & 3.6 & H8b-H8c & 4.2 \\
H3a,5a-H12c & 5.3 & H10b-H12b & 4.6 \\
H7a-H8a & 2.8 & H10b-H2c,6c & 3.5 \\
H7a-H10a & 4.4 & H10b-H3c,5c & 4.6 \\
H7a-H8b & 4.3 & H10b-H8c & 3.9 \\
H7a-H12b & 5.2 & H12b-H2c,6c & 3.3 \\
H7a-H7c & 4.1 & H12b-H3c,5c & 5.0 \\
H7a-H8c & 4.1 & H12b-H7c & 2.5 \\
H7a-H12a & 5.1 & H12b-H8c & 4.0 \\
H8a-H10a & 2.9 & H12b-H10c & 3.7 \\
H8a-H8b & 4.2 & H2c,6c-H7c & 2.8 \\
H8a-H8c & 4.5 & H2c,6c-H8c & 2.6 \\
H8a-H12c & 4.7 & H2c,6c-H10c & 3.1 \\
H10a-H12a & 4.3 & H3c,5c-H7c & 3.9 \\
H10a-H8c & 4.6 & H3c,5c-H8c & 3.8 \\
H10a-H12c & 4.4 & H3c,5c-H10c & 4.2 \\
H12a-H7b & 4.7 & H7c-H8c & 3.1 \\
H2b,6b-H7b & 3.0 & H7c-H10c & 2.4 \\
H2b,6b-H8b & 2.8 & H8c-H10c & 3.5 \\
H2b,6b-H10b & 3.4 & H10c-H12c & 3.8 \\
\hline
\end{tabular}


the NOE analysis, the smallest $Q$ factor of 0.04 was found for SRSSRSSR (or its enantiomer; Figure 4.12 and Figure A.5), this confirms the relative configuration beyond reasonable doubt. In order to fully define the conformation, which as mentioned is essential for correct calculation of chiroptical properties, we included four dipolar couplings of the rotatable phenyl rings. Calculation of the RDCs in each of the aromatic rings from the previously described conformation satisfied the experimental RDCs for ring a (C1a-C6a), but not for ring b (C1b-C6b) and c (C1c-C6c; Figure 4.13a). This confirmed that the NOE data cannot be used to determine the conformation of ring $\mathrm{b}$ and $\mathrm{c}$ reliably. Accordingly, we performed a molecular mechanics conformational search (BEST method in Discovery Studio 2.5) and two different torsion angles for each aromatic ring $b$ and c could be found, amounting to four conformers (Figure 4.14). After optimizing with DFT at the B3LYP/6-31G(d) level using the IEFPCM solvent continuum model with DMSO as the solvent, all $16{ }^{1} D_{\mathrm{CH}}$ back-calculated RDCs matched the experimental ones and the populations of the different conformations were calculated under the assumption that the different torsion angles of the phenyl rings are not correlated with each other (Figure $4.13 \mathrm{~b})$. In the RDC analysis, since the shape of different conformers is highly similar, the relative orientation of conformers in the ensemble was determined by superimposing each to the mean structure.
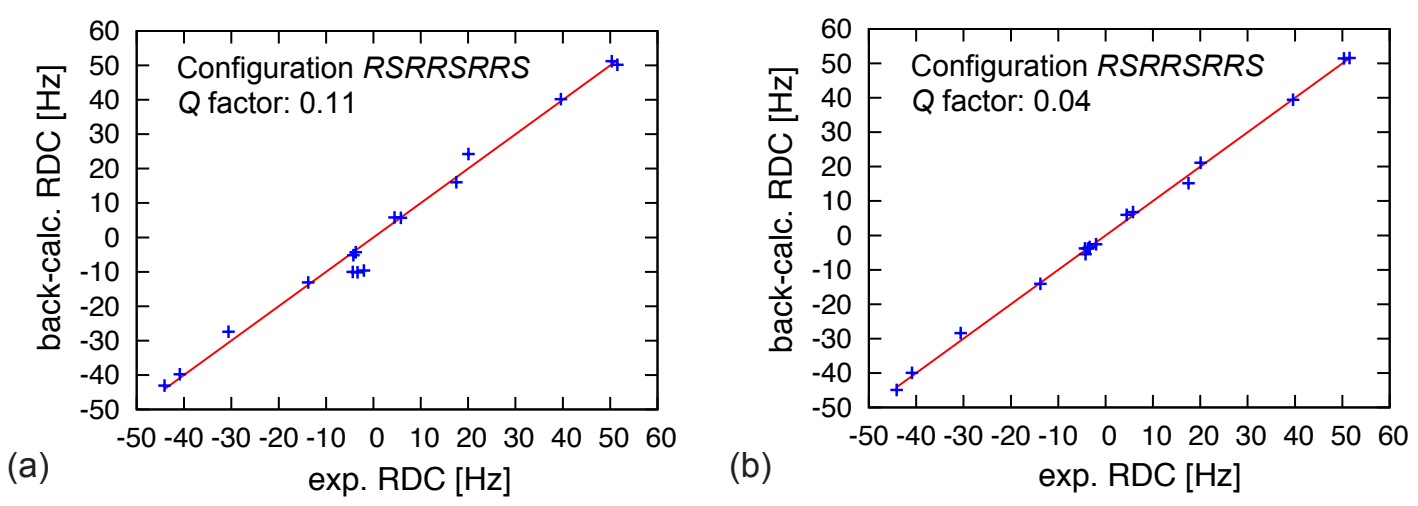

Figure 4.13: Correlation between experimental and back-calculated RDCs using (a) only one orientation of the flexible aromatic ring systems, (b) four different orientations of the flexible aromatic ring systems (rings b and c) found by the conformational search based on molecular mechanics calculations.

\subsubsection{Elucidation of the absolute configuration by chiroptics}

Having established the conformation and relative configuration of vatiparol by RDCand NOE-based NMR spectroscopy measurements, chiroptical methods were em- 


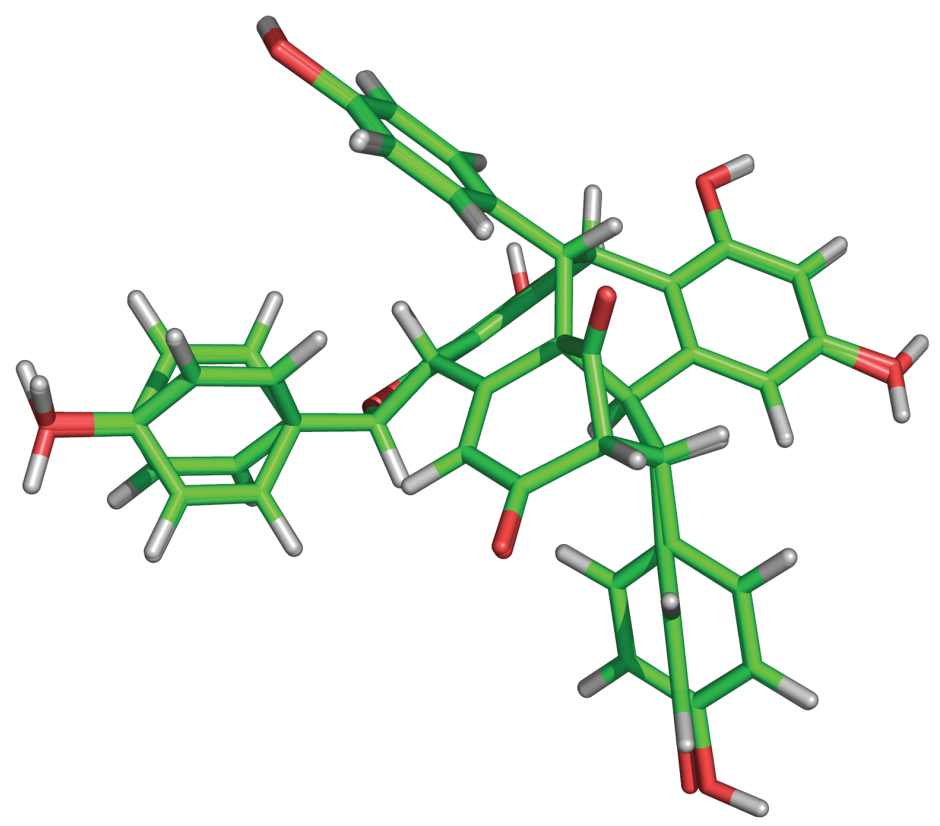

\begin{tabular}{|c|c|c|c|}
\hline Conformer & $\begin{array}{c}H 7 b-C 7 b- \\
C 1 b-C 6 b / 2 b\end{array}$ & $\begin{array}{c}\mathrm{H} 7 \mathrm{c}-\mathrm{C} 7 \mathrm{c}- \\
\mathrm{C} 1 \mathrm{c}-\mathrm{C} 6 \mathrm{c} / 2 \mathrm{c}\end{array}$ & Population \\
\hline 1 & $5^{\circ}$ & $-18^{\circ}$ & $26 \%$ \\
\hline 2 & $66^{\circ}$ & $-18^{\circ}$ & $39 \%$ \\
\hline 3 & $5^{\circ}$ & $-76^{\circ}$ & $14 \%$ \\
\hline 4 & $66^{\circ}$ & $-76^{\circ}$ & $21 \%$ \\
\hline
\end{tabular}

Figure 4.14: Conformation of vatiparol with two different orientations of the rotatable aromatic rings $\mathrm{C} 1 \mathrm{~b}-\mathrm{C} 6 \mathrm{~b}$ and $\mathrm{C} 1 \mathrm{c}-\mathrm{C} 6 \mathrm{c}$ each. The configuration is SRSSRSSR. The table gives the dihedral angles which define aromatic ring orientations for the four conformations and their populations assuming no correlation of the torsion angle of the phenyl rings $b$ and $\mathrm{c}$.

ployed to elucidate the absolute configuration. Computation of the ECD spectra for the RDC determined conformers were performed with time-dependent DFT at the B3LYP/6-31G(d) level. Figure 4.15a shows that the calculated ECD spectra of the four RDC determined conformers are significantly different. Linear combination with the populations determined from the RDC analysis yields the theoretical curve (Figure 4.15b), this indicates the importance of the accurately determined conformations from the RDC data. This theoretical ECD spectrum was compared with the experimental one recorded for a wavelength range of 250-400 $\mathrm{nm}$. The ECD spectrum calculated for SRSSRSSR agrees very well with the measured one (Figure 4.15b). Three calculated intense Cotton effects at 245, 272, and $291 \mathrm{~nm}$ could be 
assigned to the experimental Cotton effects at 253, 290, and $309 \mathrm{~nm}$, respectively, whereas the two negative Cotton effects of $R S R R S R R S$ could not be assigned in the experimental spectrum. When the calculated spectrum of SRSSRSSR is shifted to lower wavelengths by $15 \mathrm{~nm}$ (Figure 4.15c) [Giorgio et al., 2005, Di Bari et al., 2005, Berova et al., 2007], it becomes more obvious that both sign and shape of the measured spectrum can be very well reproduced by the calculated one. In order to verify the ECD results, we measured the ORD values at four different wavelengths in DMSO for vatiparol, and calculated them for the four possible conformations with DFT at the B3LYP/6-31G(d) level. Figure 4.15d shows that the calculated ORD curves for the different conformations of SRSSRSSR have the same sign and reproduce the experimental curve very well. Considering the concurrent results from ORD and ECD together, beyond reasonable doubt the absolute configuration of vatiparol is: $\mathrm{C} 7 \mathrm{a} S, \mathrm{C} 8 \mathrm{a} R, \mathrm{C} 7 \mathrm{~b} S, \mathrm{C} 8 \mathrm{~b} S, \mathrm{C} 12 \mathrm{~b} R, \mathrm{C} 14 \mathrm{~b} S, \mathrm{C} 7 \mathrm{c} S$ and $\mathrm{C} 8 \mathrm{c} R$.

To rationalize the biochemical construction of the novel carbon skeleton for vatiparol, a biosynthetic pathway has been proposed based on the computations of reactivity of the tautomeric resveratrol radicals by our collaborators from Nanjing University. The obtained results are shown in Figure A.6 and [Ge et al., 2012]. Furthermore, by using a motif-inspired biological assessment strategy, vatiparol was shown to possess selective inhibitory effect on the expression of monocyte chemoattractant protein 1 (MCP-1, also known as CCL2), which has been reported to be an attractive target for anti-inflammatory agents [Gu et al., 2000].

\subsubsection{Conclusion}

In conclusion, we have determined the relative configuration and accurate conformation of vatiparol with RDC enhanced NMR spectroscopy with only $1 \mathrm{mg}$ of the sample in a $1.7 \mathrm{~mm}$ NMR tube. We have proposed a reproducible and efficient way for preparing the slim-gels for $1.7 \mathrm{~mm}$ NMR tubes. Based on the NMR determined conformation, the absolute configuration was elucidated by a comparison of the DFT calculated and experimental ECD and ORD spectra. The presented methodology and strategy will be generally useful for the determination of structure of novel natural compounds with limited availability. In this study we have recorded the spectrum under anisotropic conditions with a "room temperature" probe. By using a $1.7 \mathrm{~mm}$ (or less) cryogenically cooled probe, it can be expected that the required analyte amount could be further reduced to as little as $300 \mu \mathrm{g}$, which is at the submicromolar level. 

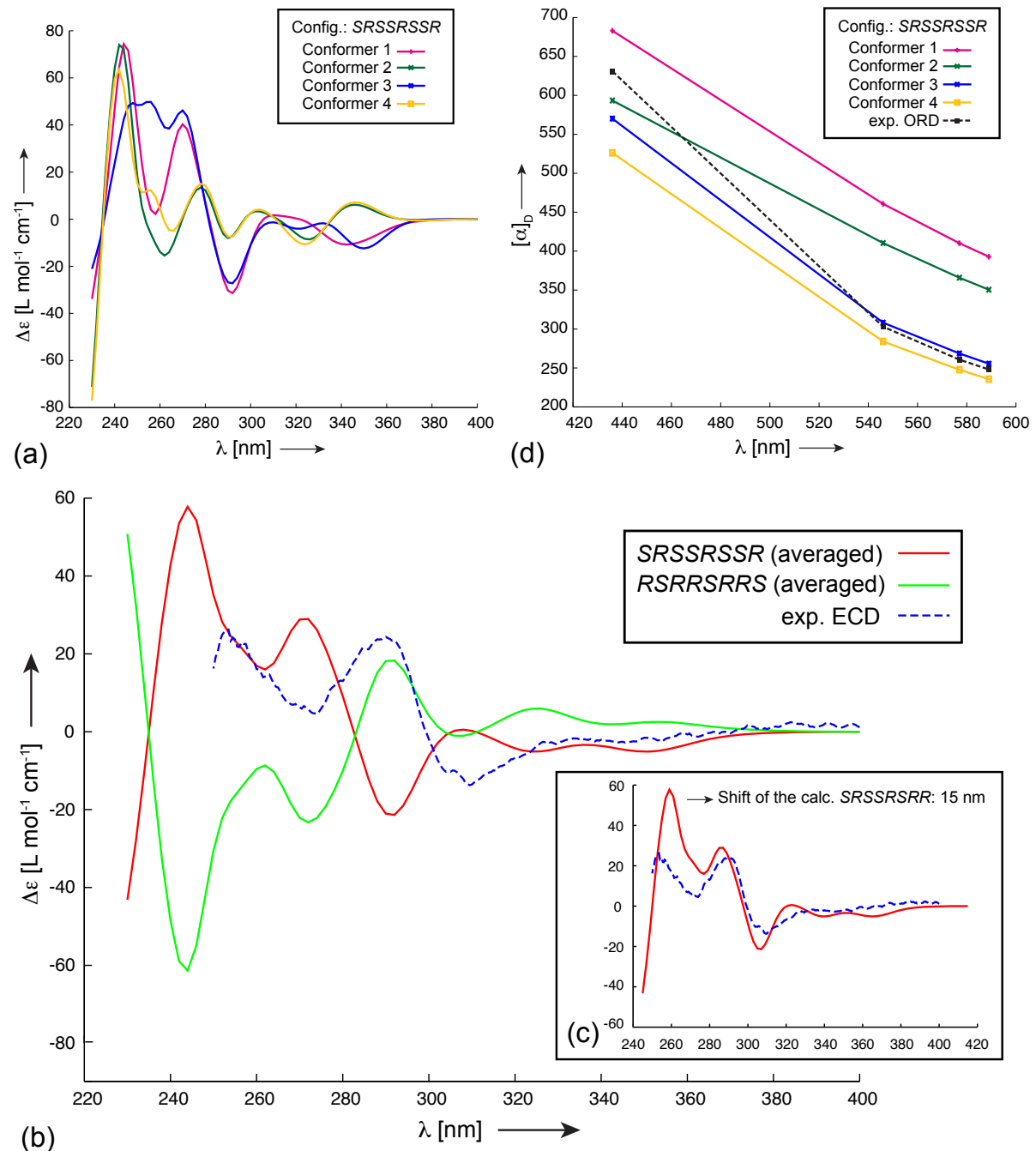

(b)

Figure 4.15: Comparison of the computed and acquired ECD/ORD curves for vatiparol. (a) Comparison of calculated ECD spectra of four RDC-determined conformers. (b) Comparison of experimentally measured ECD spectrum of vatiparol in DMSO to the linearly weighted averages calculated from the RDC-derived ensemble for both enantiomers SRSSRSRR and RSRRSRSS. (c) A $15 \mathrm{~nm}$ shift to higher wavelength of the calculated ECD spectrum of $S R S S R S R R$ results in a good fit to the experimental spectrum. (d) Comparison of experimental ORD values of vatiparol in DMSO (dashed line) to those calculated (solid lines) for the RDC determined conformers. All calculations were performed with DFT at the B3LYP/6-31G(d) level using the IEFPCM solvent continuum model with DMSO as the solvent. 


\subsection{Determination of the absolute configuration of naphth 1 and naphth 2}

\subsubsection{Introduction}

Two new naphthoquinone epoxides, 2,3-epoxy-2,3-dihydrolapachol (naphth 1) and 2,3-epoxy-2,3-dihydro-8-hydroxylapachol (naphth 2), both not previously isolated from natural sources, were identified from Barleria alluaudii by our collaborators Dr. Emily L. Whitson and Dr. Tawnya C. McKee from the Center for Cancer Research, National Cancer Institute (NCI), Frederick, USA. B. alluaudii, a plant endemic to Madagascar, was investigated for the first time as part of an ongoing search for synergistic TRAIL (tumor necrosis factor- $\alpha$-related apoptosis-inducing ligand) sensitizers. The newly isolated compounds naphth 1 and naphth 2 (Figure 4.16 ), similarly to many other members of the lapachol family of quinones, show TRAIL sensitization effects. $\beta$-Lapachol was evaluated by the NCI in the clinic during the 1970s, but was later withdrawn due to high levels of toxicity [Cragg et al., 2009]. The closely related $\beta$-lapachone is currently in Phase II clinical trials for advanced solid tumors [Cragg et al., 2009, Ravelo et al., 2004].

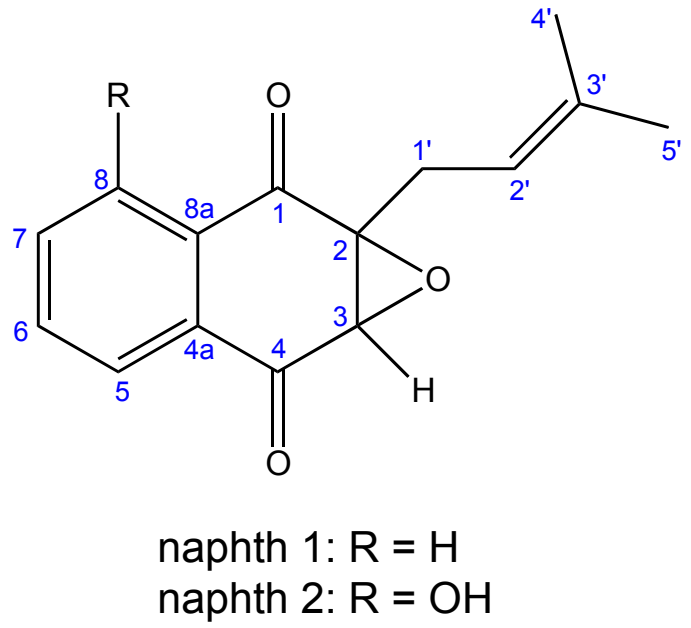

Figure 4.16: Structure and atom numbering of naphth1 and naphth2.

The structures of naphth 1 and naphth 2, as shown in Figure 4.16, only differ in the substituent at $\mathrm{C} 8$. There are two unknown stereocenters at $\mathrm{C} 2$ and $\mathrm{C} 3$, whose relative configuration can be established as $(2 R, 3 S)$ or $(2 S, 3 R)$ due to the geometrical restriction. The aim of our study is to determine the absolute configuration of naphth 1 and naphth 2 with the aid of chiroptical methods. 


\subsubsection{Elucidation of the absolute configuration by chiroptics}

In order to determine the absolute configuration of naphth 2 by chiroptical methods, it is essential to perform an analysis of the conformation of the molecule. In this study, a conformational search using molecular mechanics calculations yielded 14 possible conformers within a $10 \mathrm{kcal} / \mathrm{mol}$ energy threshold. The low-field shifted chemical shift of the hydroxyl proton in phenol suggests a hydrogen bonding between this proton and the quinone oxygen, therefore before the computation the hydroxyl proton was manually rotated so that the hydrogen bond was formed. The geometries of naphth 2 were optimized at the B3LYP/6-31G(d) level affording six possible conformers within a $1 \mathrm{kcal} / \mathrm{mol}$ energy difference (naphth 2a-2f, Figure 4.17, Table A.8). In agreement with the energy calculations, experimental proton-carbon long-range couplings also indicate a conformational averaging around the dihedrals C3-C2-C1'C2' and C2-C1'-C2'-C3' (Table A.7 and A.8). However, due to the conformational complexity and lack of experimental data, the population of the individual conformers cannot be determined by NMR. Here the conformational distributions have been calculated using the relative energies, relative zero point energies, relative Gibbs free energies based on a Boltzmann distribution, and are given in Table 4.9.

The ECD calculations for naphth 2a-2f were then conducted with the B3LYP6-31G(d) basis set using the IEFPCM solvent continuum model with methanol as the solvent (Figure A.7). The calculated ECD spectra, weighted based on the Gibbs free energy, of both the $(2 S, 3 R)$ and $(2 R, 3 S)$ enantiomers, along with the experimental ECD spectrum for naphth 2, are shown in Figure 4.18. The weighted ECD spectrum for the $(2 S, 3 R)$ enantiomer is in good accordance with the experimental ECD spectrum. In particular, the positive Cotton effect at $380 \mathrm{~nm}$ and the negative Cotton effect at $330 \mathrm{~nm}$ clearly follow the ECD pattern for naphth 2. Also it is important to note that the computed ECD spectra for individual conformers are very similar in the region of $250 \mathrm{~nm}$ to $400 \mathrm{~nm}$ (Figure A.7). This finding indicates that the weighted spectrum in this region would not change significantly if the computed populations were not accurately predicted, which is indeed the case for many systems using DFT-based energy calculations [Wodrich et al., 2007]. Comparison between experimental ORD values and the calculated ones for both possible enantiomers using DFT at three different wavelengths further supports the $(2 S, 3 R)$ configuration (Figure 4.19, Table A.9). Here all conformers except conformer 3 show positive ORD curves, which is in agreement with the experimental data. Taken all together, the absolute configuration of naphth 2 was established as $(2 S, 3 R)-2,3-$ epoxy-2,3-dihydro-8-hydroxylapachol. 

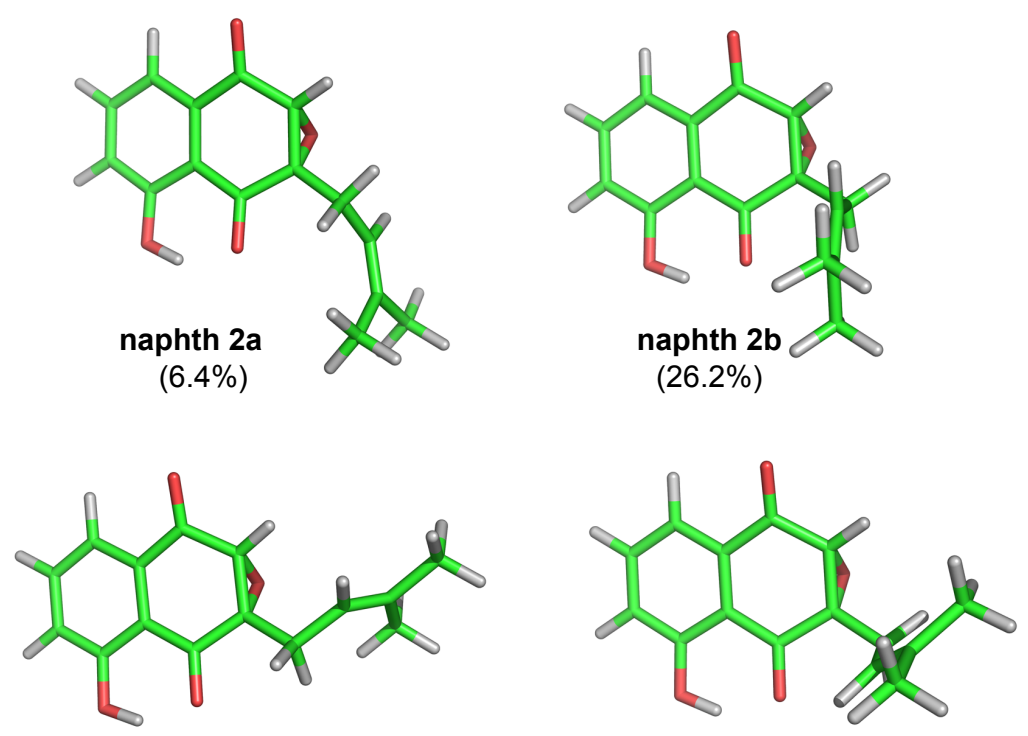

naphth 2c

$(15.4 \%)$

naphth 2d

$(10.5 \%)$
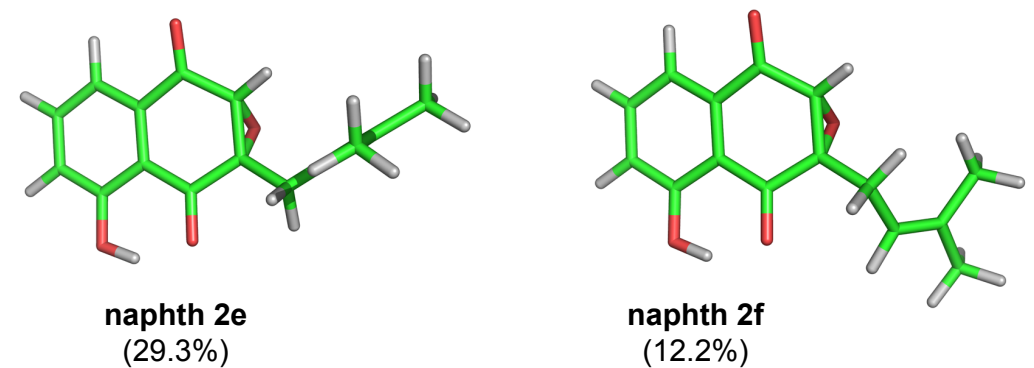

Figure 4.17: Six lowest energy conformers of $(2 S, 3 R)$-2,3-epoxy-2,3-dihydro-8hydroxylapachol (naphth 2) with their populations calculated using the Gibbs free energy.

The absolute configuration of naphth 1 was established by comparison of experimental and TD-DFT calculated ECD spectra. Following the approach outlined above, geometry optimization of naphth 1 resulted in six possible conformers within a $1 \mathrm{kcal} / \mathrm{mol}$ energy difference (naphth 1a-1f, Figure A.9, Table A.10). A comparison of the observed ECD spectrum for naphth 1 with spectra calculated by TD-DFT for the $(2 S, 3 R)$ and $(2 R, 3 S)$ enantiomers is seen in Figure 4.20. The weighted ECD spectrum for the $(2 S, 3 R)$ enantiomer is in good accordance with the experimental ECD for naphth 1. Interestingly, the optical rotations for naphth 1 and 2 are of opposite sign $(\lambda=589 \mathrm{~nm}$ : naphth $1,-9.0$; naphth $2,+92.8)$. Naphth 1 was subjected to chiral HPLC analysis to determine whether a mixture of enantiomers was present, and a single peak was observed on six chiral columns. The ORD values for the $(2 S, 3 R)$ and $(2 R, 3 S)$ enantiomers of naphth 1 were also calculated using DFT at three different wavelengths (Figure 4.21, Table A.11). Individually, the conformer values are not small, but since half of the conformers are of opposite sign, the average 
Table 4.9: Conformational analysis of naphth 2 using the IEFPCM solvent continuum model (methanol). Relative energy, relative zero point energy, and relative Gibbs free energy were calculated at the B3LYP/6-31G(d) level, respectively ( $\mathrm{kcal} / \mathrm{mol}$ ). Room temperature $(298.15 \mathrm{~K})$ was considered in the calculation for the Gibbs free energy. Conformational distribution was calculated based on the Boltzmann distribution.

\begin{tabular}{clrlrlr}
\hline Conformer & $\begin{array}{c}\Delta E \\
\left(\mathrm{kcal} \cdot \mathrm{mol}^{-1}\right)\end{array}$ & $\begin{array}{c}p_{E} \\
(\%)\end{array}$ & $\begin{array}{c}\Delta E_{0} \\
\left(\mathrm{kcal} \cdot \mathrm{mol}^{-1}\right)\end{array}$ & $\begin{array}{c}p_{E_{0}} \\
(\%)\end{array}$ & $\begin{array}{c}\Delta G \\
\left(\mathrm{kcal} \cdot \mathrm{mol}^{-1}\right)\end{array}$ & $\begin{array}{c}p_{G} \\
(\%)\end{array}$ \\
\hline 2a & 1.02 & 5.0 & 1.33 & 3.1 & 0.89 & 6.4 \\
2b & 0.05 & 25.9 & 0.04 & 27.4 & 0.07 & 26.2 \\
2c & 0.38 & 14.8 & 0.37 & 15.7 & 0.38 & 15.4 \\
2d & 0.21 & 19.7 & 0.30 & 17.8 & 0.61 & 10.5 \\
2e & 0.00 & 28.2 & 0.00 & 29.3 & 0.00 & 29.3 \\
2f & 0.88 & 6.4 & 0.87 & 6.7 & 0.52 & 12.2 \\
\hline
\end{tabular}
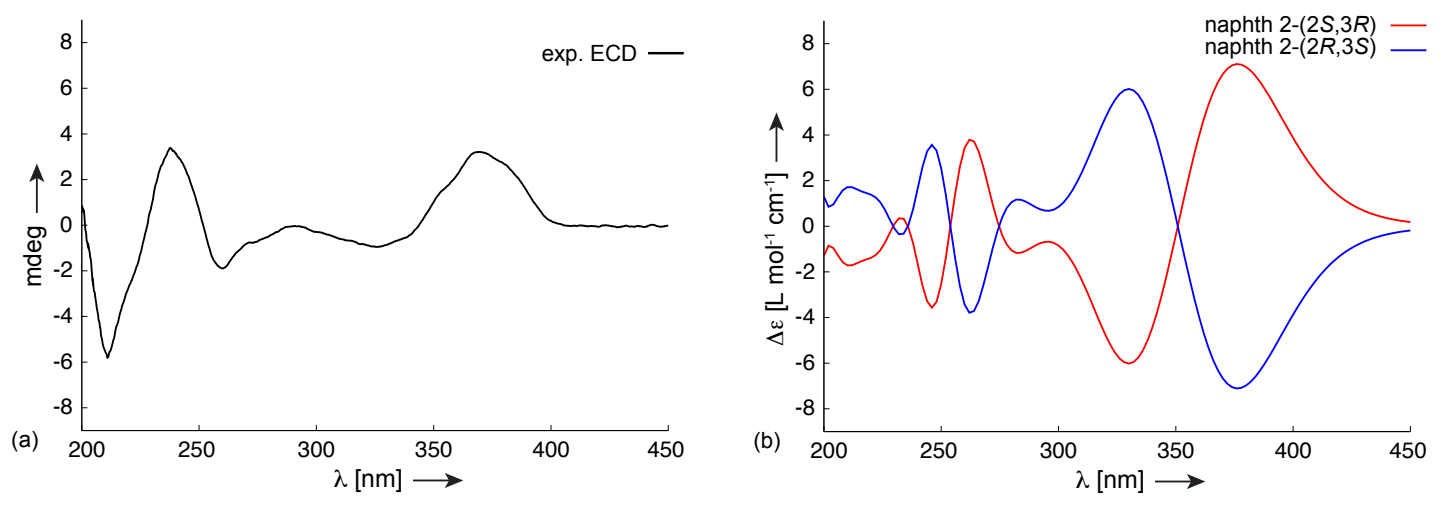

Figure 4.18: Comparison of (a) experimental ECD values with (b) those calculated for the two possible enantiomers $(2 S, 3 R$ and $2 R, 3 S)$ of naphth 2 . The calculations were performed with DFT at the B3LYP/6-31G(d) level using the IEFPCM solvent continuum model with methanol as the solvent. The calculated ECD values are weighted based on the Gibbs free energy.

values become much smaller. In this case, the calculated population of the individual conformers, derived from the energy calculation using the DFT method, determines the correct prediction of the ORD values. A small error in the energy calculation can therefore change the sign of the calculated ORD. As will be shown in Chapter 4 on the example of an Michael addition product, the correct ensemble with accurate population from the NMR analysis approximating the full conformational space of the molecule is essential for the calculation of the chiroptical properties. Furthermore, according to [McCann and Stephens, 2006], the ORD values are not reliable if the absolute value of the difference between calculated and experimental for both enantiomers is less than 74.0. Considering all these points, in the case of naphth 1 , 


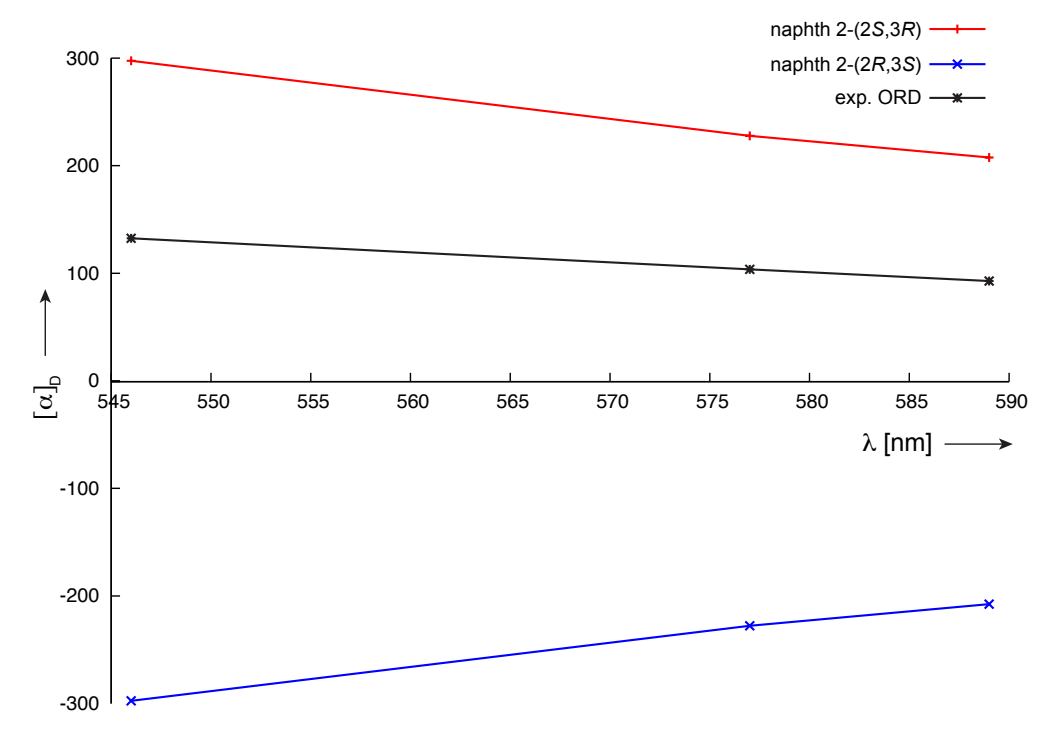

Figure 4.19: Comparison of experimental ORD values with those calculated for the two possible enantiomers $(2 S, 3 R$ and $2 R, 3 S)$ of naphth 2 . The calculations were performed with DFT at the B3LYP/6-31G(d) level using the IEFPCM solvent continuum model with methanol as the solvent. The calculated ORD values are weighted based on the Gibbs free energy.

the calculated averaged ORD values are not reliable. The absolute configuration of naphth 1 was established here as $(2 S, 3 R)$-2,3-epoxy-2,3-dihydrolapachol by the good agreement between the $(2 S, 3 R)$ calculated and experimental ECD spectra. This is in agreement with the biosynthetic principles considering naphth 1 and naphth 2 having the same $(2 S, 3 R)$ absolute configuration, because they were concurrently isolated from the same organism.

\subsubsection{Conclusion}

In summary, the absolute configuration of the 2,3-epoxy-2,3-dihydrolapachol (naphth 1 ) and 2,3-epoxy-2,3-dihydro-8-hydroxylapachol (naphth 2) that were isolated from $B$. alluaudii for the first time, has been determined using TD-DFT calculations of chiroptics. Since chiroptical properties depend on the overall molecular stereochemistry that includes the conformation as well as configuration, a careful analysis of the conformations was performed for both molecules using molecular mechanics based conformational search, followed by DFT optimizations. The ECD and ORD spectra of individual conformers have been calculated and compared. For naphth 2, the similarity of the calculated ECD pattern between $250 \mathrm{~nm}$ and $400 \mathrm{~nm}$ for all conformers, as well as the positive ORD curves for all conformers except conformer 

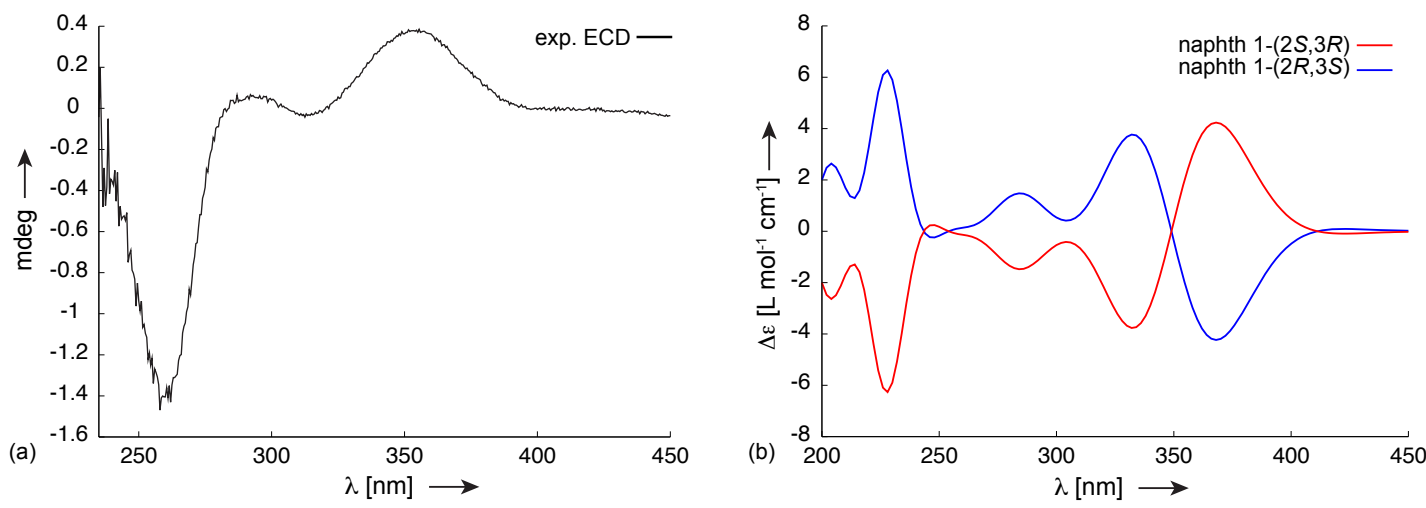

Figure 4.20: Comparison of (a) experimental ECD values with (b) those calculated for the two possible enantiomers $(2 S, 3 R$ and $2 R, 3 S)$ of naphth 1 . The calculations were performed with DFT at the B3LYP/6-31G(d) level using the IEFPCM solvent continuum model with methanol as the solvent. The calculated ECD values are weighted based on the Gibbs free energy.

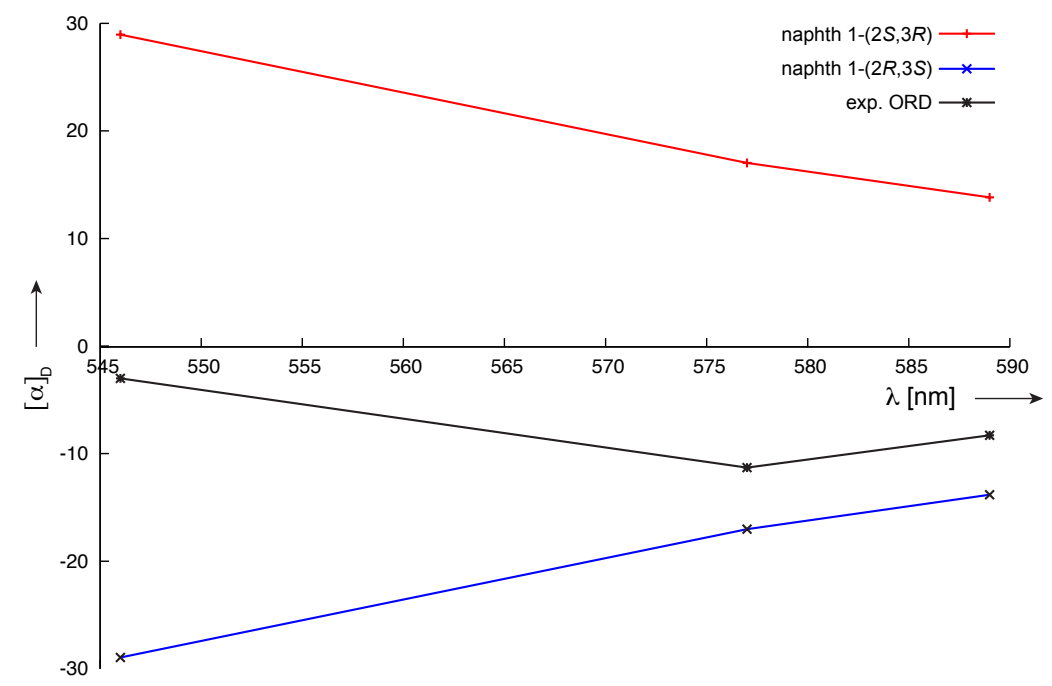

Figure 4.21: Comparison of experimental ORD values with those calculated for the two possible enantiomers $(2 S, 3 R$ and $2 R, 3 S)$ of naphth 1 . The calculations were performed with DFT at the B3LYP/6-31G(d) level using the IEFPCM solvent continuum model with methanol as the solvent. The calculated ORD values are weighted based on the Gibbs free energy.

3, indicate that the weighted spectrum has a low dependence on the accuracy of the DFT-derived populations. By comparison of the experimental ECD and ORD spectra with the weighted ones, the absolute configuration of naphth 2 was determined as $(2 S, 3 R)$. For naphth 1 , the weighted ECD spectrum for the $(2 S, 3 R)$ 
enantiomer is also in good accordance with the experimental ECD spectrum. However the experimental ORD cannot be reproduced by the weighted data of $(2 S, 3 R)$, which is due to the fact that the ORD values of half of the conformers are of opposite sign and therefore the precision of the calculated population determines the correct prediction of the ORD values. Nevertheless, the averaging of the calculated ORD values is corroborated with the 10 times smaller absolute value of experimental ORD of naphth 1 compared to naphth 2. To sum up, the comparison of experimental and computed ECD spectra determines the absolute configuration of naphth 1 as $(2 S, 3 R)$, matching with the prediction based on biosynthesis pathways. 


\section{Chapter 5}

\section{Stereochemical elucidation of molecules of class B1}

\subsection{Introduction}

Molecule class B1 has been defined for those molecules, in which the stereochemical domains do not communicate, however are connected by a relatively rigid linker (Figure 1.2). In many cases, since the stereochemical domains cannot be related by short-range experimental data such as $J$-couplings and NOEs, it is difficult to establish the relative configuration of the molecules of class B1 unambiguously with such conventional NMR methods. Because of this, the configuration of many organic molecules in this class still remains undetermined, especially either when conformational flexibility exists within one stereochemical domain or when two stereogenic domains are relatively far apart. Residual dipolar couplings make it possible to correlate different domains based on the angular information of the internuclear vectors, and therefore have the great potential for the determination of the configuration of these molecules. In this chapter two molecules of class B1 are considered, with each molecule posing different difficulties and challenges for the elucidation of their configuration using RDC-enhanced techniques.

In the first part of this chapter, the determination of absolute configuration of the product of a new Michael addition, synthesized via a niobium(V) chiral enolate, is described. As this Michael addition product contains several rotatable bonds, the conformational averaging constitutes an immense challenge for the configurational study. In this contribution, a new fundamental methodology overcoming the flexibility issue to resolve the absolute configuration will be presented. Rather than sampling the entire conformational space, ensembles of structures derived by NMR spectroscopy, which reproduce the experimental NMR spectroscopic data will be 
used to predict chiroptical properties of the molecule in solution. The technique involves two steps: residual dipolar coupling (RDC)-enhanced NMR spectroscopy to enable the determination of the conformational ensemble and the relative configuration; and subsequent prediction of ORD spectra by DFT calculations to differentiate between enantiomers. We will show that the ORD values for the different conformations in the ensemble vary tremendously and, therefore, the determination of a faithful ensemble representing the situation in solution is mandatory for calculating correct ORD values with DFT. By using the new methodology we determine the relative configuration of the reaction product, reveal with this an unexpected stereoselectivity and provide an insight into mechanistic organic chemistry.

In the second part, the determination of the relative configuration of a newly identified macrolide, comp.540a, will be presented. Since the configuration of eight centers needed to be studied, a combination of restrained MD (r-MD) simulations with the floating chirality method was proposed to determine the unknown configuration of the stereocenters simultaneously. Herein, experimental $J$-couplings and NOEs were employed as restraints, so that snapshots with the correct configuration were present with higher frequency during the MD simulations. With an energy filter that selects only a certain fraction of the snapshots with the lowest deviation energy, the stereochemical possibilities were remarkably reduced. Finally, RDC fitting to the obtained ensemble from the NOE- and $J$-restrained MD simulation was utilized to select the correct configuration from the remaining possible configurations. Furthermore, with the help of RDC-based analysis not only the relative configuration of the stereogenic centers in the backbone, but also the correct orientation and relative configuration between lactone ring and sugar could be determined unambiguously. Using this study as an example, we show that the conformation and a large number of unknown stereocenters of a complex natural product can be precisely and correctly determined using RDC-enhanced r-MD simulations with the floating chirality method.

\subsection{Determination of the absolute configuration of a Michael addition product}

\subsubsection{Introduction}

The molecule of interest in this study is the product of a new Michael addition, synthesized via a niobium(V) chiral enolate. The conjugated addition to $\alpha, \beta$ - 
unsaturated compounds (Michael reaction) is one of the most important reactions in C-C bond formation. A few examples that use metallic enolates prepared from chiral and achiral oxazolidinones in conjugated addition reactions have been described to deliver versatile intermediates in the synthesis of compounds with interesting pharmacological activities [Yura et al., 1987, Mukaiyama et al., 1987, Yura et al., 1988, Evans et al., 1990,Evans et al., 1991, Bach et al., 1995]. Based on the previously established methodology for preparing niobium enolates of oxazolidinones [Andrade, 2004,Andrade and Rocha, 2006], a study of the conjugated addition of these enolates to chalcones has been performed by our collaborators Prof. Rafael Oliveira Rocha and Prof. Carlos Kleber Z. Andrade from Instituto de Química, Universidade de Brasília, Brasília-DF, Brazil. The niobium(V)-catalyzed reaction is shown in Figure 5.1. To our knowledge, this is the first example of an addition of a chiral oxazolidinone enolate to chalcones. Furthermore, when $\mathrm{TiCl}_{4}$ was used as the Lewis acid under similar conditions no reaction was observed between these educts demonstrating the importance of $\mathrm{NbCl}_{5}$ in this process. The Michael addition product $(R)-\mathbf{3}$ was isolated with a yield of $72 \%$. The identity of this adduct was confirmed by NMR spectroscopy and a high 95:5 ratio of two diastereomers was determined by integration of the methyl signals in the ${ }^{1} \mathrm{H}$ NMR spectrum of the crude product. Since this Michael addition reaction can in principle lead to four possible diastereomers shown in Figure 5.2, our goal was to determine the configuration of the main product.

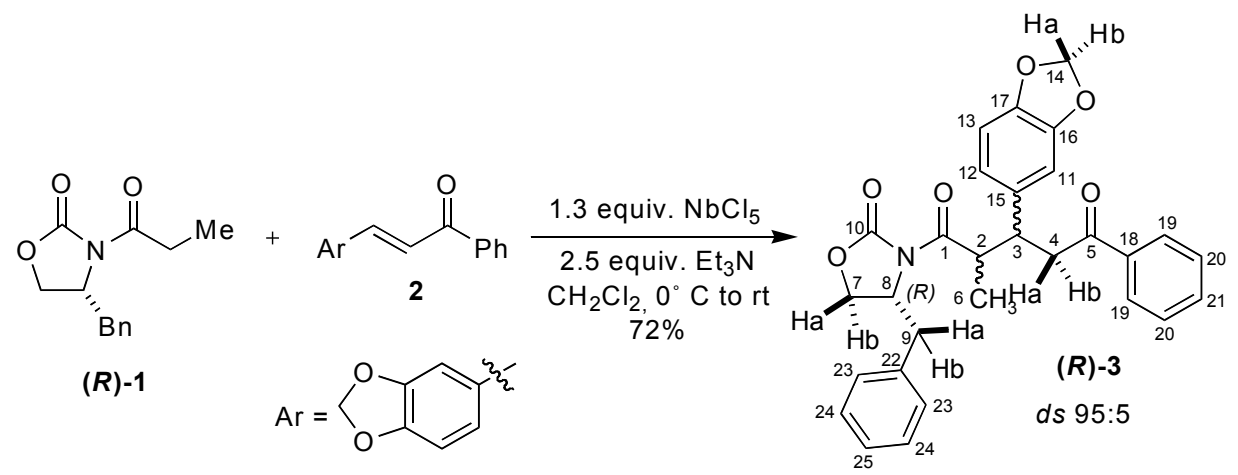

Figure 5.1: Stereoselective conjugated addition of the niobium enolates to chalcone; d.r. $=$ diastereomeric ratio. 


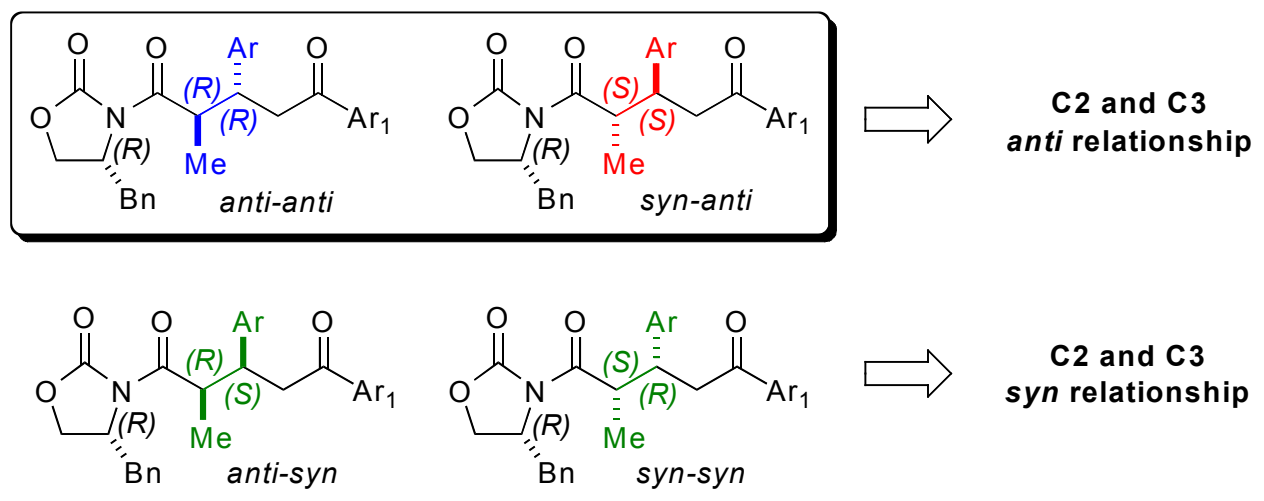

Figure 5.2: Possible diastereomers from the Michael addition of the niobium (V) enolate of oxazolidinone $(R)-\mathbf{1}$ to chalcone $\mathbf{2}$. Top left: $(R, R, R)-\mathbf{3}$, top right: $(R, S, S)-\mathbf{3}$, bottom left: $(R, R, S) \mathbf{- 3}$, bottom right: $(R, S, R)-\mathbf{3}$.

\subsubsection{Elucidation of the relative configuration and conforma- tion by NMR}

\section{NMR resonance assignment}

The ${ }^{1} \mathrm{H}$ and ${ }^{13} \mathrm{C}$ chemical shifts of the Michael addition product $(R)-\mathbf{3}$ in DMSO$d_{6}$ were assigned by means of $1 \mathrm{D}^{1} \mathrm{H}, \mathrm{COSY}$, TOCSY, ROESY,$\left[{ }^{1} \mathrm{H},{ }^{13} \mathrm{C}\right]-\mathrm{HSQC}$ and $\left[{ }^{1} \mathrm{H},{ }^{13} \mathrm{C}\right]$-HMBC NMR spectra and are listed in Table 5.1. The ${ }^{15} \mathrm{~N}$ chemical shift was assigned using a $\left[{ }^{1} \mathrm{H},{ }^{15} \mathrm{~N}\right]-\mathrm{HMBC}$ NMR spectrum. All NMR spectra were recorded at $25^{\circ} \mathrm{C}$.

\section{Measurements of scalar and residual dipolar couplings}

To elucidate the relative configuration of the two unknown stereocenters in the major product, it is necessary to perform a $J$-coupling analysis for the $\mathrm{C} 2$-C3 bond. ${ }^{3} J_{\mathrm{CH}}$ couplings were measured by $\left[{ }^{1} \mathrm{H},{ }^{13} \mathrm{C}\right]$-HECADE and $\left[{ }^{1} \mathrm{H},{ }^{13} \mathrm{C}\right]$-robust-HMBC experiments, whereas the ${ }^{3} J_{\mathrm{HH}}$ couplings were extracted from both an E.COSY spectrum and $a^{1} \mathrm{H}$ spectrum apodized with a Lorentz-to-Gauss window function.

For the RDC measurements, polymer media used to align $(R)$-3-anti were a PHgel [Haberz et al., 2005] and a PAN-gel [Kummerlöwe et al., 2007], whose properties and preparations are described in Table A.13. The RDCs were taken from $t_{2}$-coupled $\left[{ }^{1} \mathrm{H},{ }^{13} \mathrm{C}\right]$-HSQC spectra by superimposing and fitting $\omega_{2}$ traces from isotropic and anisotropic spectra. In total, two different RDC data sets were obtained, as given in Table 5.4. 
Table 5.1: Carbon and proton chemical shifts of $(R)-3-a n t i$ in DMSO- $d_{6}$ at $298 \mathrm{~K}$.

\begin{tabular}{|c|c|c|c|}
\hline Carbon & Chemical shift (ppm) & Proton & Chemical shift (ppm) \\
\hline $\mathrm{C} 1$ & 175.3 & & \\
\hline $\mathrm{C} 2$ & 42.8 & $\mathrm{H} 2$ & 4.13 \\
\hline $\mathrm{C} 3$ & 42.4 & H3 & 3.75 \\
\hline \multirow[t]{2}{*}{$\mathrm{C} 4$} & 39.9 & $\mathrm{H} 4 \mathrm{a}$ & 3.34 \\
\hline & & $\mathrm{H} 4 \mathrm{~b}$ & 3.62 \\
\hline C5 & 198.7 & & \\
\hline C6 & 14.8 & H6 & 1.15 \\
\hline \multirow[t]{2}{*}{$\mathrm{C} 7$} & 65.9 & $\mathrm{H} 7 \mathrm{a}$ & 4.26 \\
\hline & & $\mathrm{H} 7 \mathrm{~b}$ & 4.05 \\
\hline $\mathrm{C} 8$ & 54.4 & H8 & 4.59 \\
\hline \multirow[t]{2}{*}{ C9 } & 36.6 & H9a & 2.50 \\
\hline & & $\mathrm{H} 9 \mathrm{~b}$ & 2.63 \\
\hline $\mathrm{C} 10$ & 153.3 & & \\
\hline $\mathrm{C} 11$ & 108.9 & H11 & 6.95 \\
\hline $\mathrm{C} 12$ & 121.8 & $\mathrm{H} 12$ & 6.77 \\
\hline $\mathrm{C} 13$ & 108.0 & H13 & 6.77 \\
\hline \multirow[t]{2}{*}{$\mathrm{C} 14$} & 100.9 & $\mathrm{H} 14 \mathrm{a}$ & 5.92 \\
\hline & & $\mathrm{H} 14 \mathrm{~b}$ & 5.88 \\
\hline $\mathrm{C} 15$ & 137.2 & & \\
\hline $\mathrm{C} 16$ & 147.2 & & \\
\hline $\mathrm{C} 17$ & 145.8 & & \\
\hline C18 & 137.1 & & \\
\hline C19 & 128.2 & H19 & 7.95 \\
\hline $\mathrm{C} 20$ & 128.9 & $\mathrm{H} 20$ & 7.50 \\
\hline $\mathrm{C} 21$ & 133.4 & H21 & 7.61 \\
\hline $\mathrm{C} 22$ & 135.7 & & \\
\hline $\mathrm{C} 23$ & 129.6 & H23 & 6.86 \\
\hline $\mathrm{C} 24$ & 127.0 & H24 & 7.20 \\
\hline $\mathrm{C} 25$ & 128.6 & H25 & 7.22 \\
\hline
\end{tabular}

Table 5.2: Nitrogen chemical shift of $(R)-3$-anti in DMSO- $d_{6}$ at $298 \mathrm{~K}$.

\begin{tabular}{ll}
\hline Nitrogen & Chemical shift (ppm) \\
\hline $\mathrm{N}$ & 149.9 \\
\hline
\end{tabular}


Table 5.3: Long-range proton-proton and proton-carbon coupling constants of $(R)$-3-anti in DMSO.

\begin{tabular}{lrll}
\hline Atoms & ${ }^{3} J_{\mathrm{HH}}(\mathrm{Hz})$ & Atoms & ${ }^{3} J_{\mathrm{CH}}(\mathrm{Hz})$ \\
\hline H2-H3 & 8.6 & H2-C4 & 2.7 \\
H3-H4a & 4.0 & H2-C15 & $1-2$ \\
H3-H4b & 10.0 & H3-C1 & $1-2$ \\
H8-H7a & 8.0 & H3-C5 & $1-2$ \\
H8-H7b & 3.0 & H3-C6 & 3.7 \\
H8-H9a & 7.3 & H4a-C2 & 1.0 \\
H8-H9b & 3.4 & H4b-C2 & 2.0 \\
& & H8-C22 & $3-4$ \\
& & H9a-C7 & 4.9 \\
& & H9b-C7 & 6.5 \\
\hline
\end{tabular}

Table 5.4: Experimental residual dipolar couplings of $(R)$-3-anti in two alignment media.

\begin{tabular}{lrl}
\hline CH-vector & ${ }^{1} D_{\mathrm{CH}}(\mathrm{Hz})$ in PH-gel & ${ }^{1} D_{\mathrm{CH}}(\mathrm{Hz})$ in PAN-gel \\
\hline C2-H2 & -2.0 & 26.4 \\
C3-H3 & 0.0 & 26.9 \\
C7-H7a & -5.5 & -3.3 \\
C7-H7b & 7.1 & -25.5 \\
C8-H8 & 5.2 & 21.9 \\
C9-H9a & -3.2 & \\
C9-H9b & 5.2 & \\
C11-H11 & -0.4 & 19.4 \\
C12-H12 & 1.7 & 10.8 \\
C13-H13 & -0.5 & 17.5 \\
C14-H14a & -2.5 & -7.5 \\
C14-H14b & 2.1 & -16.3 \\
C21-H21 & 1.6 & -17.5 \\
C25-H25 & 3.1 & -15.1 \\
\hline
\end{tabular}




\section{Configurational analysis}

The Michael adduct $(R)-\mathbf{3}$ contains three stereocenters. The one located in the oxazolidinone ring (C8) is known to have an $R$ configuration. To elucidate the relative configuration of the two unknown stereocenters $\mathrm{C} 2$ and $\mathrm{C} 3$ in the major product, all measured long-range $J$-couplings were translated into dihedral angles with the calibration of an Altona-equation [Haasnoot et al., 1980]. Comparison of the dihedral angles with possible conformations in Newman projections of the C2-C3 bond establishes the anti relative configuration for compound ( $R$ )-3-anti (Figure 5.3). Therefore, the possible diastereomers are reduced from four to just two $(R, R, R)-\mathbf{3}$ and $(R, S, S)-\mathbf{3}$, in which the stereocenters correspond to $\mathrm{C} 8, \mathrm{C} 2$, and $\mathrm{C} 3$, respectively (Figure 5.2).

Since the two neighboring unknown stereocenters and the known stereocenter in the oxazolidinone ring are separated by two fully and one partially rotatable bonds, long-range structure restraints were required to find the correct configuration. Such restraints were provided by quantitative ROEs, as well as by RDCs. A total number of 40 ROEs were derived from a ROESY experiment with $400 \mathrm{~ms}$ mixing time. A total of 36 local and remote ROEs (Table 5.5) were then used as the restraints in the following structure evaluation.

Due to the flexibility of this molecule, conformational averaging constitutes a methodological challenge. In this study, to sample the conformational space we calculated a molecular dynamics trajectory for each diastereomer in vacuo. The MD-simulation was carried out at $298 \mathrm{~K}$ for $1 \mathrm{~ns}$ using the Discover software. A single structure was logged every 1 ps so that a trajectory with 1000 structures was obtained. Then 10000 ensembles, each of 10 conformations, were generated for the diastereomers $(R, R, R)-\mathbf{3}$ and $(R, S, S)-\mathbf{3}$. These 10 structures were randomly picked from the 1000 snapshots of the molecular dynamics trajectory.

The ROEs and RDCs were used to rank the selected ensembles of both diastereomers according to the violation of the ROEs (Equation 3.8) and the quality of the fit to the RDCs ( $Q$ factor, Equation 3.5). The full ROE and RDC analysis was implemented in the program relax. For each ensemble the ROE violation was averaged to the minus $6^{\text {th }}$ power over all members of the ensemble. The RDC fitting for each ensemble was performed independently for the two sets of RDC data (from a PH-gel and a PAN-gel) by calculating the alignment tensor using the Nelder-Mead simplex optimization method [Nelder and Mead, 1965]. The ten structures in an ensemble were assumed to have equal populations. In this study, we use the assumption that 
Table 5.5: Proton distance information derived from ROESY spectrum at $400 \mathrm{~ms}$ mixing time. All distances were referenced to the methylene protons $\mathrm{H} 9 \mathrm{a} / \mathrm{H} 9 \mathrm{~b}$, which was assumed to have a proton distance of $1.78 \AA$.

\begin{tabular}{llll}
\hline Atoms & Distance $(\AA)$ & Atoms & Distance $(\AA)$ \\
\hline H2-H3 & 2.7 & H2-H4a & 3.5 \\
H2-H4b & 3.1 & H2-H6 & 2.0 \\
H2-H8 & 4.6 & H2-H9a & 4.1 \\
H2-H11 & 3.3 & H2-H12 & 3.1 \\
H3-H6 & 2.5 & H3-H11 & 2.7 \\
H3-H12 & 2.6 & H3-H19 & 3.4 \\
H4a-H6 & 2.2 & H4a-H11 & 4.7 \\
H4a-H19 & 2.7 & H4b-H6 & 3.6 \\
H4b-H11 & 2.9 & H4b-H12 & 2.9 \\
H4b-H19 & 2.9 & H6-H8 & 4.8 \\
H6-H11 & 3.8 & H6-H12 & 3.8 \\
H6-H19 & 4.3 & H7a-H8 & 2.6 \\
H7b-H8 & 3.1 & H7b-H9b & 3.3 \\
H7b-H23 & 2.8 & H8-H9a & 2.7 \\
H8-H9b & 2.7 & H8-H23 & 3.2 \\
H9a-H23 & 2.7 & H9b-H23 & 2.7 \\
H11-H14a & 4.3 & H11-H19 & 4.0 \\
H12-H19 & 4.7 & H14a-H24 & 4.0 \\
\hline
\end{tabular}




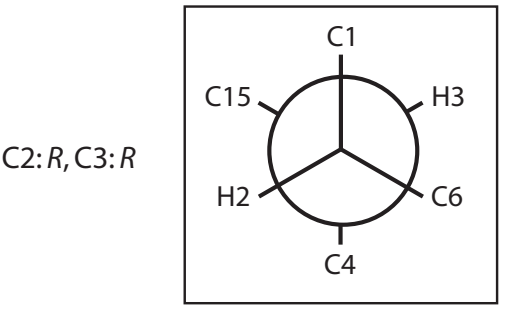

3

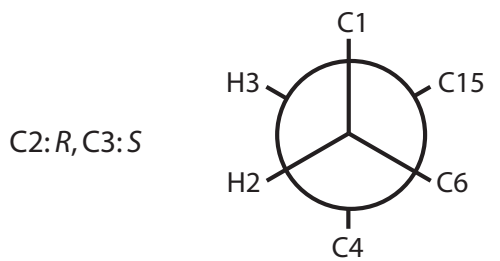

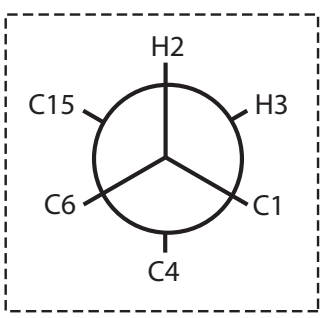

1

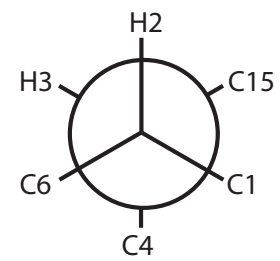

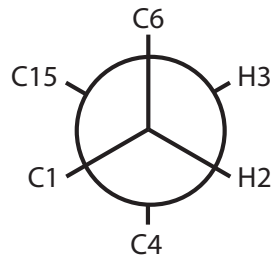

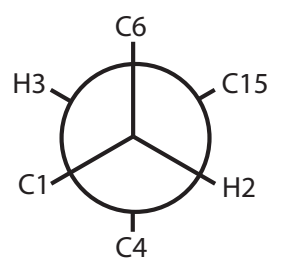

\begin{tabular}{lll}
\hline${ }^{3} J(\mathrm{H} 2-\mathrm{H} 3)$ & $8.6 \mathrm{~Hz}$ & extracted from $1 \mathrm{D}$ spectrum \\
${ }^{3} \mathrm{~J}(\mathrm{H} 3-\mathrm{C} 6)$ & $3.7 \mathrm{~Hz}$ & extracted from $\left[{ }^{1} \mathrm{H}-{ }^{13} \mathrm{C}\right]-\mathrm{HECADE}$ \\
${ }^{3} \mathrm{~J}(\mathrm{H} 3-\mathrm{C} 1)$ & $1-2 \mathrm{~Hz}$ & extracted from $\left[{ }^{1} \mathrm{H}-{ }^{13} \mathrm{C}\right]-$ robust-HMBC \\
${ }^{3} J(\mathrm{H} 2-\mathrm{C} 4)$ & $2.7 \mathrm{~Hz}$ & extracted from $\left[{ }^{1} \mathrm{H}-{ }^{13} \mathrm{C}\right]-\mathrm{HECADE}$ \\
${ }^{3} \mathrm{~J}(\mathrm{H} 2-\mathrm{C} 15)$ & $1-2 \mathrm{~Hz}$ & extracted from $\left[{ }^{1} \mathrm{H}-{ }^{13} \mathrm{C}\right]-$ robust-HMBC \\
\hline
\end{tabular}

Figure 5.3: Newman projections of 6 possible conformers for the dihedral C2-C3. The three conformers on top have a configuration of $(2 R, 3 R)$, whereas the three conformers at the bottom have a configuration of $(2 R, 3 S)$. $J$-coupling analysis suggests an anti configuration of $\mathrm{C} 2$ and $\mathrm{C} 3$. The Newman projections in the frames with solid and broken lines were determined as the major and minor conformers, respectively. The ratio of populations of the major and minor conformers is $3: 1$.

all the structures in an ensemble have the same alignment tensor [Schuetz et al., 2007, Schuetz et al., 2008]. This assumption is justified, since the overall shape of the conformations in the ensembles is very similar (Figure 5.6). To use the single alignment tensor approximation, the relative orientation of conformers in the ensemble is determined by superimposing all heavy atoms of each conformation in the ensemble to the heavy atoms of the mean structure using the program MOLMOL2.1. As opposed to previous approaches [Schuetz et al., 2007, Schuetz et al., 2008], no weighting of the RDCs and ROEs is required, which establishes a more robust way of analyzing the data. The dots in Figure 5.4 represent the ROE violation in $\AA^{2}$, the $Q$ factor for the PH-gel data and the $Q$ factor for the PAN-gel data for each ensemble. From Figure 5.4 it is obvious that the blue $(R, R, R)-\mathbf{3}$ and the red $(R, S, S)-\mathbf{3}$ 
datasets are well resolved. The ensembles of $(R, R, R)-3$ fulfill the ROEs and RDCs significantly better than those of $(R, S, S)$-3. Furthermore, the projection of Figure 5.4 onto the PAN/PH plane is depicted in Figure 5.5. Here, less $(R, S, S)-\mathbf{3}$ ensembles are present than $(R, R, R) \mathbf{- 3}$ ones as the $(R, R, R)$-3 ensembles violate the restraints less than the $(R, S, S)-\mathbf{3}$ ensembles. This $2 \mathrm{D}$ projection clearly demonstrates that $(R, R, R)-\mathbf{3}$ is better supported by the NMR data. For all conformations and for both diastereomers, the $\mathrm{C}^{1}-\mathrm{N}$ bond is antiperiplanar.

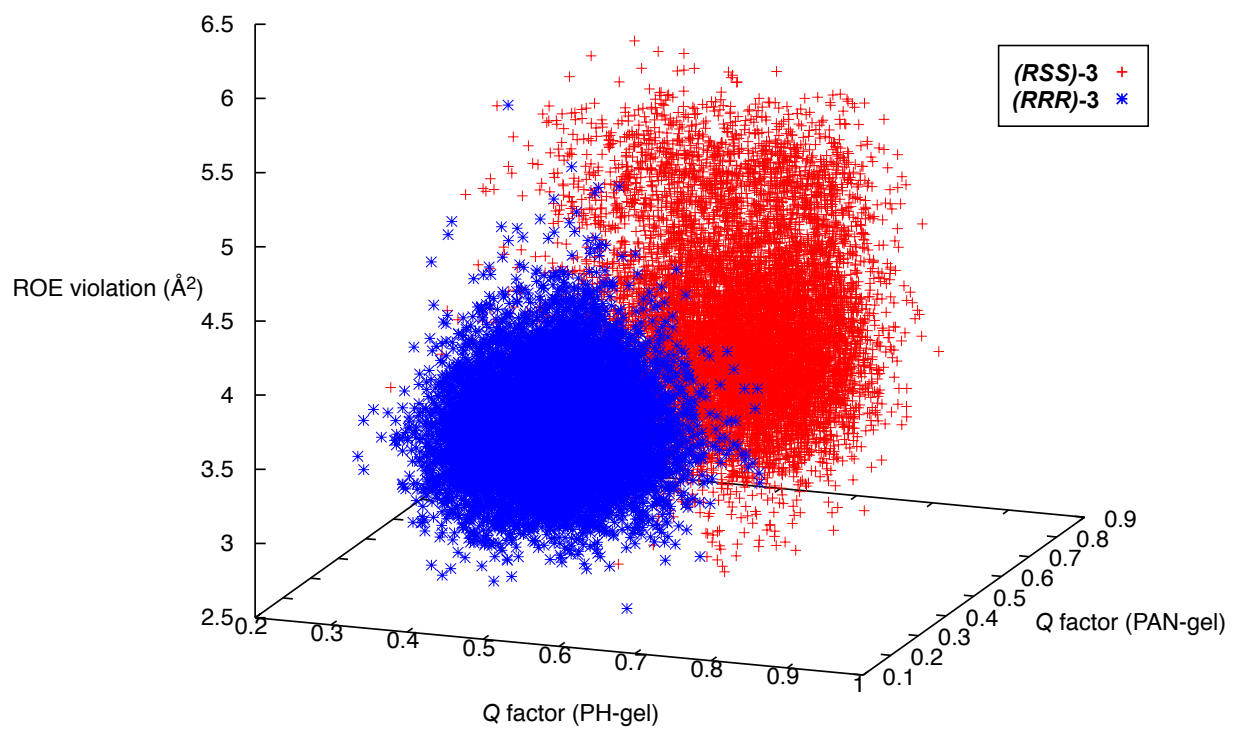

Figure 5.4: Distinguishing diastereomers by using RDCs and ROEs. The axes of the plot are the ROE violation in $\AA^{2}$, the $Q$ factor of the $\mathrm{PH}$ gel RDC data and the $Q$ factor of the PAN gel RDC data. The 10000 ensembles of 2 diastereomers are symbolized in different colors: $(R, R, R)-\mathbf{3}$ (blue) and $(R, S, S)-\mathbf{3}$ (red). 2D projections are presented in Figure A.10. For both diastereomers, the $\mathrm{C}^{1}-\mathrm{N}$ bond is antiperiplanar.

Another less robust method is to compare the best ensemble of $(R, R, R)-\mathbf{3}$ to that of $(R, S, S)-\mathbf{3}$. Here "best" is defined by a combined $Q$ factor using quadratic averaging:

$$
Q_{\text {total }}=\sqrt{Q_{\mathrm{PAN}}^{2}+Q_{\mathrm{PH}}^{2}+Q_{\mathrm{ROE}}^{2}}
$$

in which the $Q_{\mathrm{ROE}}$ was calculated using Equation 3.9. For $(R, R, R)$-3, the best ensemble has $Q$ factors of 0.17 and 0.32 for PAN and PH gel data, respectively, whereas $(R, S, S)-\mathbf{3}$ has $Q$ factors of 0.34 and 0.50 , respectively. The ROE violation of these two best ensembles is smaller than $3.5 \AA^{2}$ (Figure 5.5), corresponding to a $Q_{\mathrm{ROE}}$ factor of 0.10. Also the principal components of the alignment tensors $D_{\mathrm{xx}}$, $D_{\text {yy }}$, and $D_{\text {zz }}$ are smaller for the best ensemble of $(R, R, R)-\mathbf{3}$ than the $(R, S, S)-\mathbf{3}$ en- 


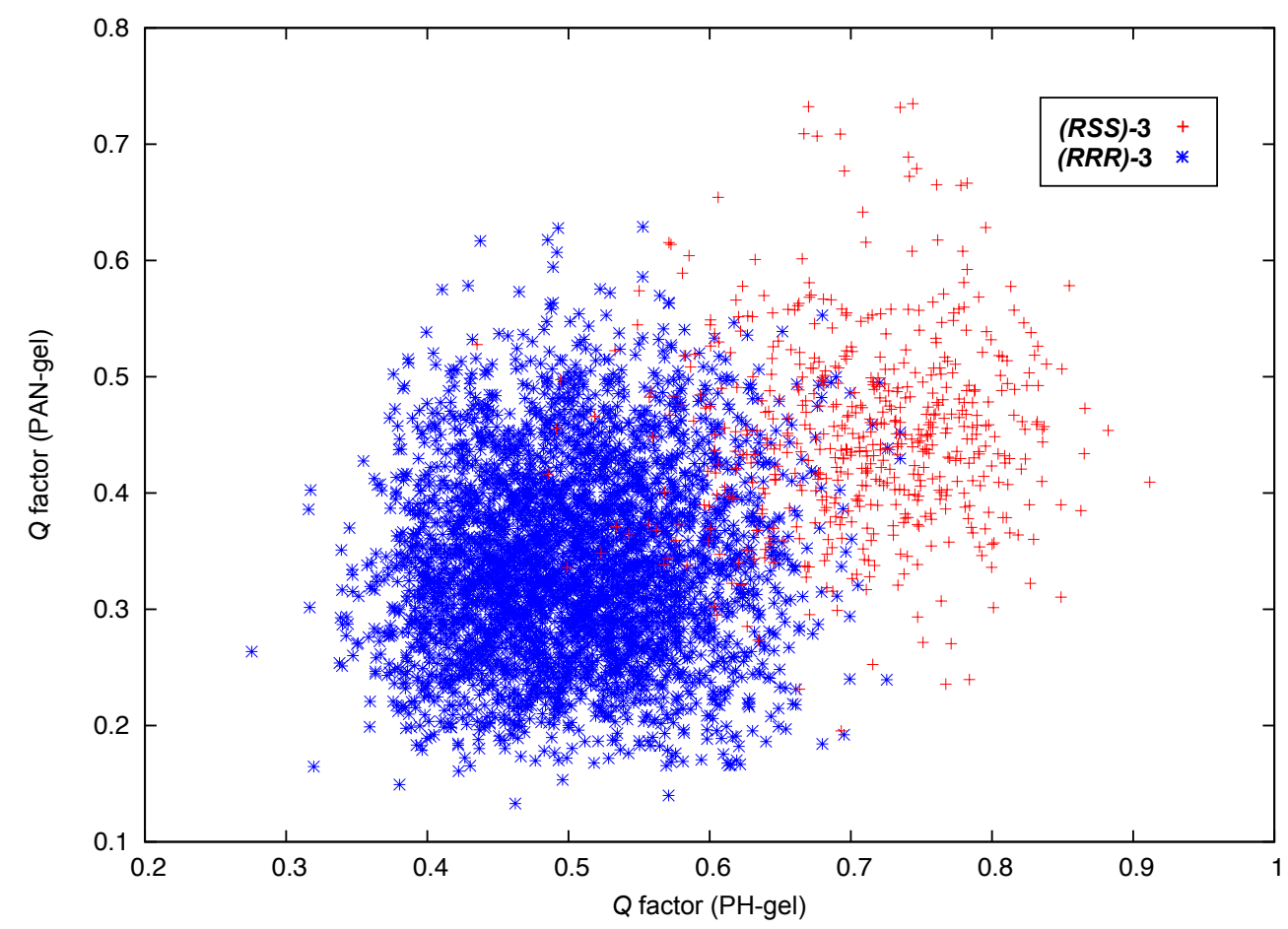

Figure 5.5: The projection of Figure 5.4 onto the PAN/PH $Q$ factor plane, only including ROE violations between 0 and $3.5 \AA^{2}$.

semble (Table 5.6). The largest RDC value of $(R)$-3-anti in the PAN gel is $26.92 \mathrm{~Hz}$, whereas that of the $\mathrm{PH}$ gel is $7.12 \mathrm{~Hz}$. In both alignment media, the $D_{z z}$ values of $(R, R, R)-\mathbf{3}$ rather than $(R, S, S)-\mathbf{3}$ are closer to the maximum experimental dipolar couplings. A similar observation has been made during the analysis of previous configurational studies [Schuetz et al., 2007], in which fitting the experimental dipolar couplings with the wrong diastereomers yielded larger alignment tensors than fitting them with the correct diastereomer. Furthermore, using the Atonal equation (Equation 2.46) a cross-validation of the experimental ${ }^{3} J$-couplings (H2-H3, H3-H4a, and H3-H4b) given in Table 5.7 against the structural models of the best ensembles was performed, suggesting that the best ensembles of both configurations $(R, R, R)-\mathbf{3}$ and $(R, S, S)-\mathbf{3}$ are in a good agreement with the experimental data (Table 5.7).

Without the presence of an organometal, the $\mathrm{C}^{1}-\mathrm{N}$ amide bond is in slow exchange on the ${ }^{1} \mathrm{H}$ NMR spectroscopy time scale at room temperature due to its double-bond character. Since we see only one set of peaks, only one of the two possible configurations is populated. Above, we assumed that the configuration is 
Table 5.6: The diagonalized alignment tensors and the $Q$ factors of the best ensembles of $(R, R, R)-\mathbf{3}$ and $(R, S, S)-\mathbf{3}$.

\begin{tabular}{llllll}
\hline Configuration & Medium & $D_{x x}(\mathrm{~Hz})$ & $D_{y y}(\mathrm{~Hz})$ & $D_{z z}(\mathrm{~Hz})$ & $Q$ factor \\
\hline$(R, R, R)-\mathbf{3}$ & PAN-gel & -37.64 & 1.07 & 37.57 & 0.17 \\
& PH-gel & -6.69 & -0.72 & 8.41 & 0.32 \\
$(R, S, S)-\mathbf{3}$ & PAN-gel & -52.05 & 2.71 & 50.35 & 0.34 \\
& PH-gel & -7.50 & -2.75 & 11.24 & 0.50 \\
\hline
\end{tabular}

Table 5.7: The measured coupling constants versus the back-calculated coupling constants of the five best ensembles of $(R, R, R)-\mathbf{3}$ and $(R, S, S)-\mathbf{3}$. For both diastereomers, the $\mathrm{C}^{1}-\mathrm{N}$ bond is antiperiplanar.

\begin{tabular}{|c|c|c|c|}
\hline Coupled protons & $\begin{array}{l}\text { Calculated } \\
(R, R, R)-\mathbf{3}\end{array}$ & $\begin{array}{c}\text { coupling }(\mathrm{Hz}) \\
(R, S, S)-3\end{array}$ & Measured coupling $(\mathrm{Hz})$ \\
\hline H2-H3 & $9.8-10.7$ & $8.1-9.3$ & 8.5 \\
\hline H3-H4a & $8.3-10.8$ & $10.3-11.1$ & 10.0 \\
\hline $\mathrm{H} 3-\mathrm{H} 4 \mathrm{~b}$ & $3.1-4.7$ & $3.3-3.8$ & 4.0 \\
\hline
\end{tabular}

$\mathrm{C}^{1}-\mathrm{N}$ antiperiplanar. We therefore repeated the above described analysis with the $\mathrm{C}^{1}-\mathrm{N}$ bond in the synperiplanar configuration and compared this with the results assuming the antiperiplanar configuration. From Figure 5.7 it is obvious that the $(R, R, R)-3\left(\mathrm{C}^{1}-\mathrm{N}\right.$ antiperiplanar $)$ configuration fulfils the $\mathrm{RDC}$ data measured in the PAN gel much better than the $(R, R, R)-\mathbf{3}\left(\mathrm{C}^{1}-\mathrm{N}\right.$ synperiplanar $)$ configuration. Also in the worse fitting configuration $(R, S, S)-\mathbf{3}$, the structures with antiperiplanar configuration show lower $Q$ factors than the ones with synperiplanar configuration (Figure A.11). The obtained result is in agreement with the literature [Lou et al., 1995, Li et al., 1994], which shows that the C-N antiperiplanar configuration is lower in energy than the $\mathrm{C}-\mathrm{N}$ synperiplanar configuration. This analysis shows again the power of RDC, which could be used to determine the relative orientation of different moieties in one molecule. In contrast to the RDC, the NOE is not very sensitive due to the flexibility of the molecule and the minus $6^{\text {th }}$ power dependence.

The stereochemical outcome $((R, R, R)-3)$ of the previously unused niobium(V)catalyzed version of the Michael addition is quite surprising. Expected transition states of this reaction based on the literature [Yura et al., 1987, Mukaiyama et al., 1987, Yura et al., 1988, Evans et al., 1990, Evans et al., 1991, Bach et al., 1995] would 

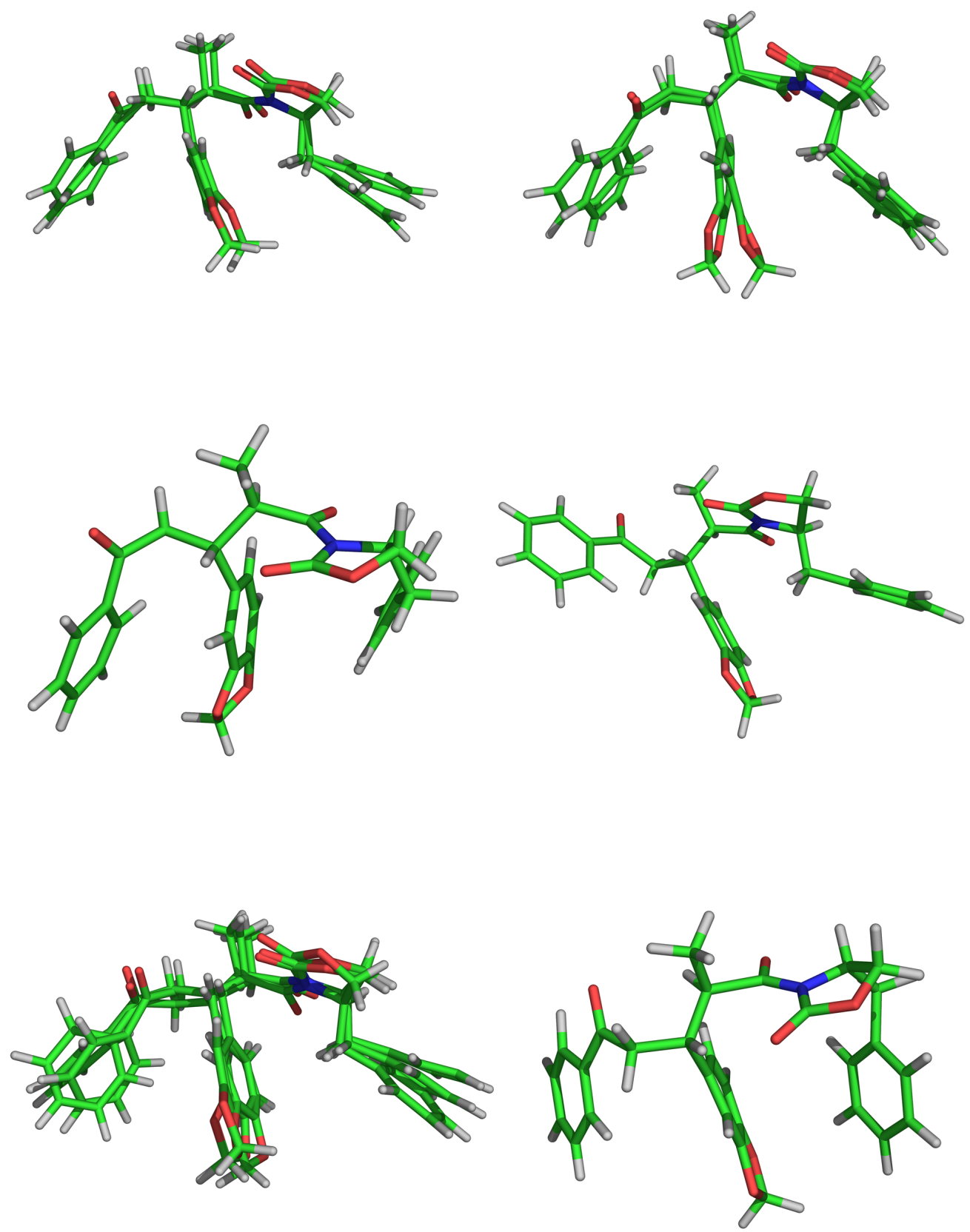

Figure 5.6: The ten structures of the best ensemble of $(R, R, R)-\mathbf{3}$. For all conformations, the $\mathrm{C}^{1}-\mathrm{N}$ is antiperiplanar.

lead to the $(R, S, S) \mathbf{- 3}$ isomer. Since the $(R, R, R)-\mathbf{3}$ isomer is formed instead, our collaborators from Brazil suggested four new transition states with antiperiplanar and synclinal attack on the $S i$ and $R e$ faces of the chalcone. In all cases, niobium is complexed to the carbonyl group of the chalcone, which promotes its activation and forms 8-membered cyclic transition states in that the endo carbonyl group of 


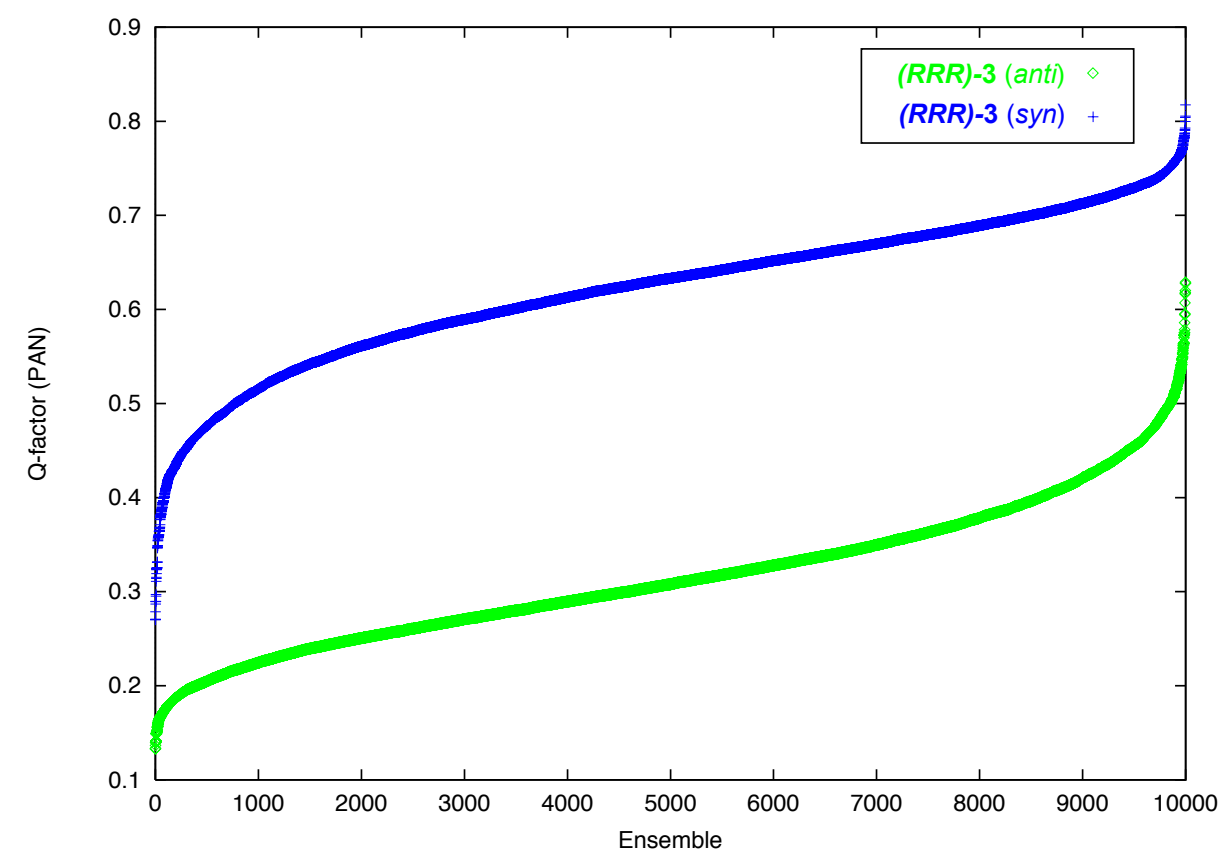

Figure 5.7: Comparison of the $Q_{\mathrm{PAN}}$ factors between the $\mathrm{C}^{1}-\mathrm{N}$ antiperiplanar (green) and $\mathrm{C}^{1} \mathrm{-N}$ synperiplanar (blue) configuration of the diastereomer $(R, R, R)-\mathbf{3}$.

the oxazolidinone is opposite to the enolate to minimize electronic effects, such as the dipole moment (Figure 5.8). The lack of activity of $\mathrm{TiCl}_{4}$ in this reaction may be due to its inability to simultaneously complex to both carbonyl groups of the oxazolidinone and the chalcone. Whether these transition states are indeed formed in this reaction remains to be seen. They are, nevertheless, chemically plausible and lead to the observed products.

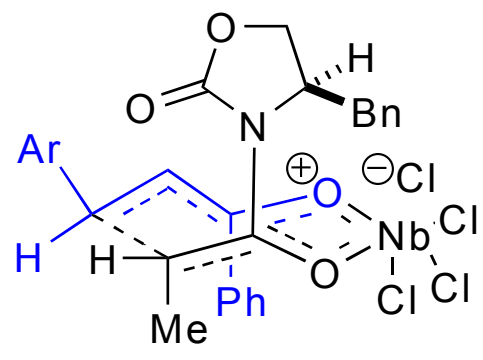

TS-A

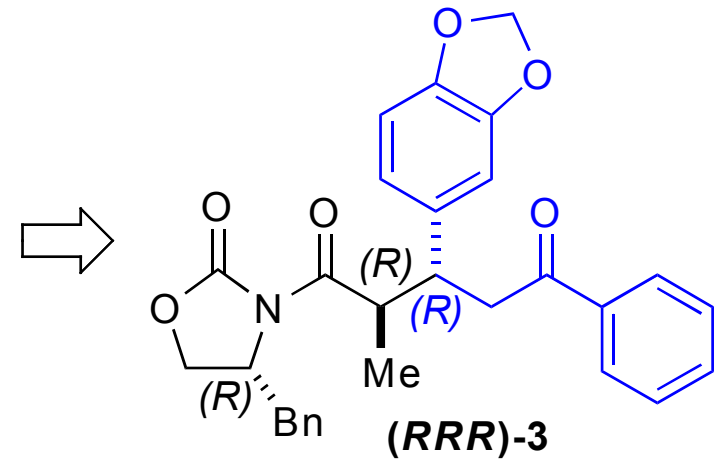

(RRR)-3

Figure 5.8: Chair-like transition state (TS) for $(R, R, R)-\mathbf{3}$. 


\subsubsection{Elucidation of the absolute configuration by ORD}

Although C8 is known to be in the $R$ configuration, since the configuration of the chiral auxiliary was known a priori, here we present the hypothetical case that the configuration of $\mathrm{C} 8$ is unknown and therefore two enantiomers $(R, R, R)-\mathbf{3}$ and $(S, S, S)-\mathbf{3}$ are possible. For completion, we also included $(R, S, S)-\mathbf{3}$ and $(S, R, R)-\mathbf{3}$ ignoring the previous determination of the relative configuration using NMR spectroscopy. This study can be considered to be a test for the accuracy of the conformational ensemble. In addition, it would establish the combination of the ensembles determined by NMR spectroscopy and chiroptical measurement as a versatile method for the determination of the absolute configuration of flexible molecules.

ORD values at 4 different wavelengths (436, 546, 578 and $589 \mathrm{~nm})$ were measured and compared with those calculated with DFT from the best ensemble of structures as determined by NMR spectroscopy. The structures of the best ensemble were firstly minimized and then DFT optimized at the B3LYP/6-31G(d) level of theory. The optical rotation dispersion calculations were performed with the optimized structures as input coordinates with the same basis sets as the optimizations using the IEFPCM solvent continuum model with DMSO as the solvent. Table A.14 and Figure 5.9 comprise the experimental and calculated ORD data measured at different wavelengths. Taking large errors in the calculated values into account, the measured negative values are compatible with both $(R, R, R)-\mathbf{3}$ and $(S, R, R)-\mathbf{3}$ configurations. Since the relative configuration of $(S, R, R)$-3 is incompatible with the NMR spectroscopic analysis, and could therefore be eliminated, the absolute configuration is unambiguously determined to be $(R, R, R)-\mathbf{3}$. This answer to the hypothetical question required no information about the configuration of the auxiliary. Thus, the finding of the configuration of the auxiliary used in the reaction, namely $R$, shows that the approach is useful for flexible molecules in solution. It should also be noted from Figure 5.10 and Tables A.15 and A.16 that the calculated ORD values of each member of the ensemble are highly divergent. Approximately half of the conformations in the ensemble yield single conformation ORD values that would suggest the $(S, S, S)$-3 configuration. Only when we use the correct ensemble of conformations and average over the individual ORD values do we get the correct answer. Hence an accurate ensemble approximating the full conformational space of the molecule is essential for the calculation of the chiroptical properties. The ensembles without NMR spectroscopy distance and RDC restraints would not be sufficient. The high dependence of the ORD values on the conformation was observed in the study of naphth 1 as well (see Chapter 4). However in this case, since the conformational distribution could not be determined experimentally, the conformationally averaged 
ORD values could not be predicted correctly.

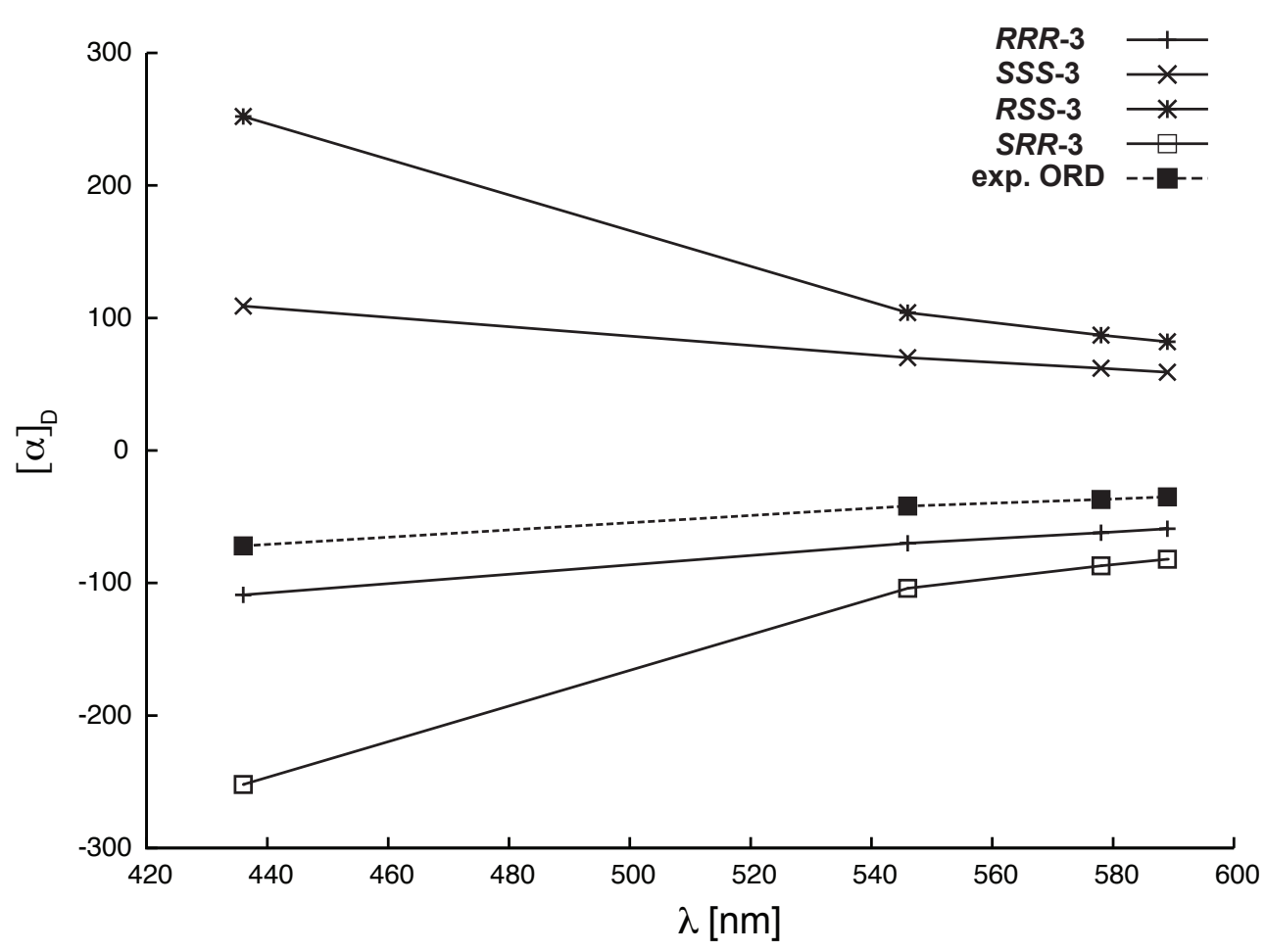

Figure 5.9: Comparison of experimental ORD values with those calculated by DFT for the four possible diastereomers of $\mathbf{3}$.

Thus, combining diastereomer differentiation by using isotropic and anisotropic NMR spectroscopy data with enantiomer differentiation by experimental validation of the DFT-calculated chiroptical properties of ensembles derived from NMR spectroscopy, the hypothetical absolute configuration question is determined as $(R, R, R)$ 3 beyond resonable doubt.

\subsubsection{Conclusion}

In conclusion, a new methodology using RDC-enhanced NMR spectroscopy was proposed to first determine the ensemble of conformations, which reveals the relative configuration by the NMR spectroscopy data and gives a faithful description of the solution ensemble. The chiroptical properties of the ensemble is then predicted by DFT and compared to experimental data to establish the absolute configuration.

Our results show that for a highly flexible molecule, such as compound $(R, R, R)$ 3 that was synthesized by a novel $\mathrm{Nb}(\mathrm{V})$-catalyzed Michael addition, the relative 


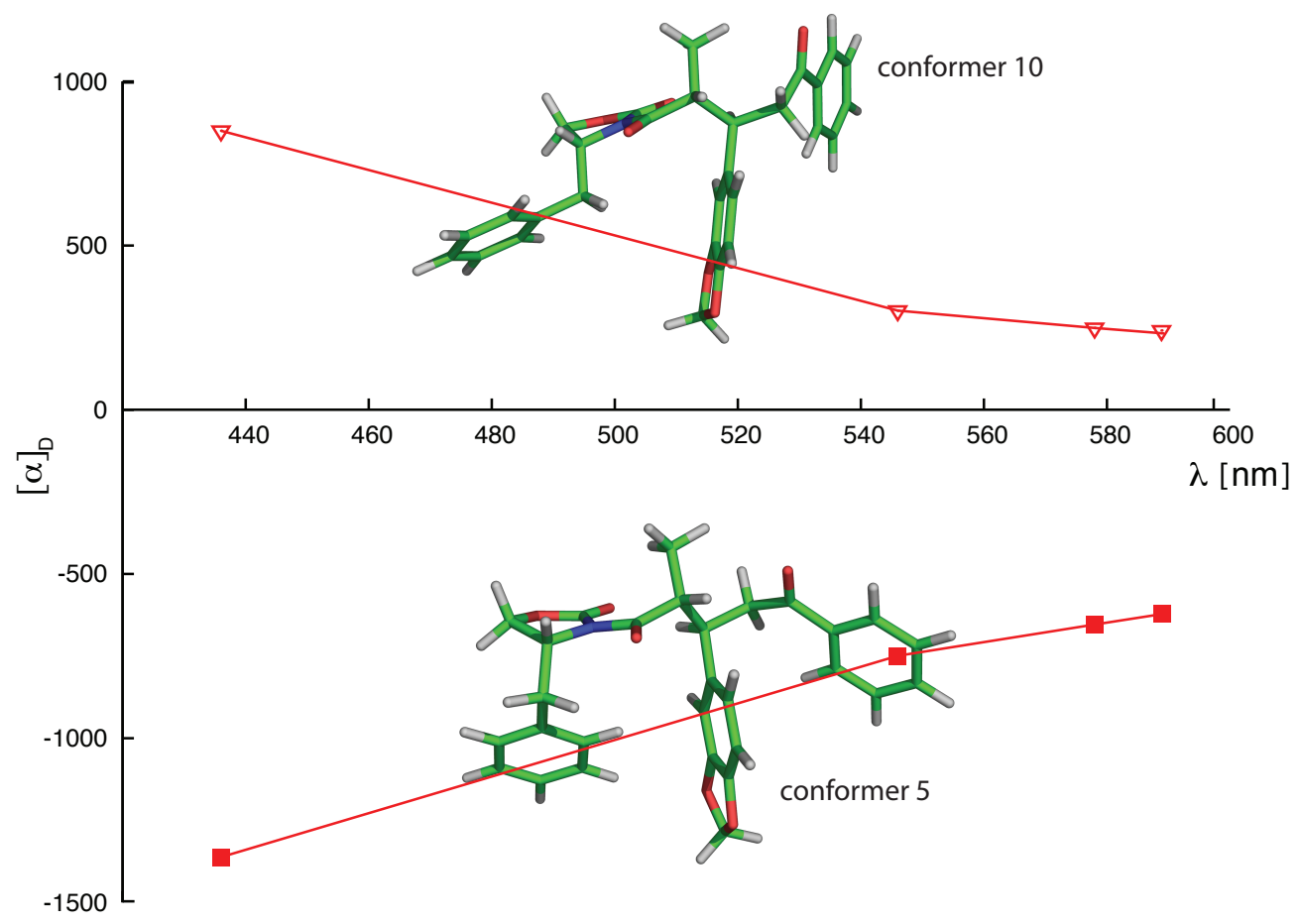

Figure 5.10: Plot of the calculated ORD values for two different conformations, selected from the best ensemble of $(R, R, R)-\mathbf{3}$.

configuration can be determined by carefully measuring $J$-couplings, NOE or ROE derived distances, and RDCs. Two different alignment media were used in this study to obtain the anisotropic RDC data set. The distance and anisotropic data are then used to select the best ensemble of 10 structures, whereby each member is obtained randomly from free molecular dynamics simulations, from a large pool of 10000 different ensembles. The fit of the RDCs used the single alignment tensor approximation for all different conformations in the ensemble. The obtained results clearly show that the ensembles with $(R, R, R)-\mathbf{3}$ configuration are in far better agreement with the experimental data than the $(R, S, S)-\mathbf{3}$ ensembles.

Extending the methodology to determine the absolute configuration without the knowledge that the configuration of $\mathrm{C} 8$ is $R$, chiroptical measurements were employed. The chiroptical properties of the ensembles selected from the NMR spectroscopic analysis were calculated by DFT and compared to measured ORD values. Since the ORD values vary tremendously when the molecule adopts different conformations, the determination of a faithful ensemble representing the situation in solution is mandatory. As a result, the hypothetical absolute configuration question is unequivocally and indisputably answered, and is $(R, R, R)-\mathbf{3}$. 


\subsection{Configurational and conformational analysis of comp.540a}

\subsubsection{Introduction}

Comp.540a (Figure 5.11) is a 16-membered macrolide that has been isolated from marine actinobacteria Solwaraspora. This compound has the same constitution in the macrocyclic lactone ring part as tylosin, which is a widely used veterinary medicine for treating bacterial infections. The absolute configuration of tylosin has been firstly determined by X-ray crystallography [Omura et al., 1980]. Here we have obtained $13 \mathrm{mg}$ of comp.540a from our collaborator Dr. Matthias Köck from Alfred-Wegener-Institut, Bremerhaven, Germany. The goal of this study is on the one hand to determine the relative configuration of the stereocenters in the macrocylic lactone ring, as well as the relative configuration between the lactone ring and the rhamnose (6-desoxy-mannose). On the other hand since macrolides are a big group of natural products that have the general property of containing a large number of stereocenters, we want to derive here a general and simple method to elucidate the configuration of these molecules on the basis of the example of comp.540a.

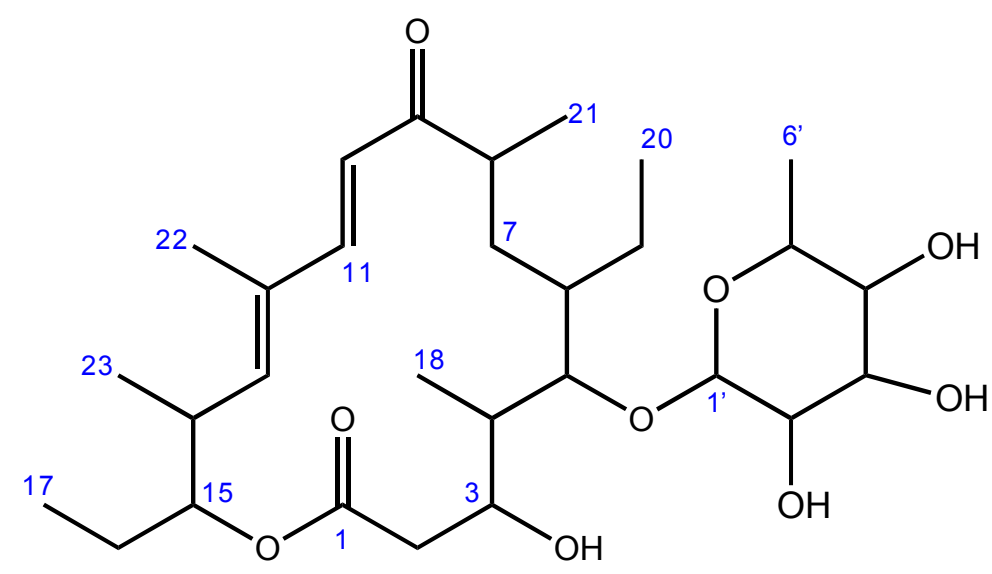

Figure 5.11: The structure of comp.540a with atom numbering.

\subsubsection{Elucidation of the relative configuration of the macrolide ring}

NMR resonances assignment

The ${ }^{1} \mathrm{H}$ and ${ }^{13} \mathrm{C}$ chemical shifts of comp.540a in DMSO- $d_{6}$ were assigned by means of 1D- ${ }^{1} \mathrm{H}, \mathrm{COSY}$, TOCSY, NOESY, $\left[{ }^{1} \mathrm{H},{ }^{13} \mathrm{C}\right]-\mathrm{HSQC}$ and $\left[{ }^{1} \mathrm{H},{ }^{13} \mathrm{C}\right]-\mathrm{HMBC}$ NMR spec- 
tra (Table 5.8). All NMR spectra were recorded at $25{ }^{\circ} \mathrm{C}$.

\section{Measurements of scalar and residual dipolar couplings}

${ }^{3} J_{\mathrm{HH}}$ couplings were extracted from an E.COSY spectrum, and long-range proton carbon couplings were derived with $\left[{ }^{1} \mathrm{H}_{-}{ }^{13} \mathrm{C}\right]$-HECADE and $\left[{ }^{1} \mathrm{H}_{-}{ }^{13} \mathrm{C}\right]-\mathrm{HSQMBC}$ experiments. All the couplings are listed in Table 5.9.

For the RDC measurements, both PH-gel and $(R)$-APhES-gel were employed to align comp.540a. $(R)$-APhES-gel has very similar properties as the PH-gel. The only change is that one of the two methyl groups in the monomer 2-Acrylamido2-methylpropane-1-sulfonic acid (AMPS) of the PH-gel were replaced by a phenyl group leading to 2-Acrylamido-2-phenylethane-1-sulfonic acid (APhES) as the building block for radical polymerization. Nevertheless, this constitutional change results in a significant difference of the alignment property [Schmidt et al., 2009], which will also be shown later in this study. Deuterium quadrupolar splittings of $3.8 \mathrm{~Hz}$ and $9.8 \mathrm{~Hz}$ were observed for PH-gel and $(R)$-APhES-gel, respectively. The two experimental RDC data sets are listed in Table 5.10

\section{Quantitative analysis of the NOEs}

Quantitative NOE build-up curves for nine different mixing times varying from 50 to $200 \mathrm{~ms}$ were derived from NOESY experiments that suppress zero-quantum artifacts. 46 NOEs were integrated at $200 \mathrm{~ms}$ mixing time and translated into proton-proton distances (Table 5.11), which were referenced to $\mathrm{H7a} / \mathrm{H} 7 \mathrm{~b}$, assuming the distance between two geminal protons to be $1.78 \AA$.

\section{Configurational analysis of the macrocyclic lactone ring}

There are 7 unknown stereocenters (C3, C4, C5, C6, C8, C14 and C15) of comp.540a, resulting in 128 stereochemical possibilities. The previously applied approach of cross-validating each possible configuration against the NMR data is in this case highly time consuming and leads to a tremendous amount of work. Better methods such as floating chirality-restrained distance geometry (DG) in combination with distance-bounds-driven dynamics (DDD), the so called "fc-rDG/DDD" method [Grube and Köck, 2007,Köck et al., 2012], would allow a simultaneous determination of the relative configuration of all stereocenters. However, since the fc-rDG/DDD 
Table 5.8: Carbon and proton chemical shifts of comp.540a in DMSO- $d_{6}$ at $298 \mathrm{~K}$.

\begin{tabular}{lcll}
\hline Carbon & Chemical shift $(\mathrm{ppm})$ & Proton & Chemical shift $(\mathrm{ppm})$ \\
\hline C1 & 172.6 & & \\
C2 & 40.9 & H2a & 1.95 \\
& & H2b & 2.43 \\
C3 & 66.1 & H3 & 3.52 \\
C4 & 42.2 & H4 & 1.54 \\
C5 & 81.4 & H5 & 3.49 \\
C6 & 40.0 & H6 & 0.92 \\
C7 & 33.6 & H7a & 1.27 \\
& & H7b & 1.78 \\
C8 & 45.4 & H8 & 2.53 \\
C9 & 203.1 & & \\
C10 & 119.2 & H10 & 6.50 \\
C11 & 147.5 & H11 & 7.08 \\
C12 & 133.5 & & \\
C13 & 146.1 & H13 & 5.61 \\
C14 & 38.8 & H14 & 2.71 \\
C15 & 78.3 & H15 & 4.56 \\
C16 & 24.5 & H16a & 1.81 \\
& & H16b & 1.51 \\
C17 & 10.2 & H17 & 0.87 \\
C18 & 9.9 & H18 & 0.84 \\
C19 & 22.3 & H19a & 1.21 \\
& & H19b & 1.45 \\
C20 & 12.8 & H20 & 0.83 \\
C21 & 17.7 & H21 & 1.16 \\
C22 & 13.1 & H22 & 1.79 \\
C23 & 16.4 & H23 & 1.01 \\
C1' & 102.9 & H2' & 4.46 \\
C2' & 71.1 & H3' & 3.72 \\
C3' & 71.4 & & \\
C4' & 72.2 & H5' & 3.20 \\
C5' & 69.7 & & 3.51 \\
C6 & 18.1 & & \\
\hline
\end{tabular}


Table 5.9: Long-range proton-proton and proton-carbon coupling constants of comp.540a in DMSO.

\begin{tabular}{|c|c|c|c|}
\hline Atoms & ${ }^{3} J_{\mathrm{HH}}(\mathrm{Hz})$ & Atoms & ${ }^{2,3} J_{\mathrm{CH}}(\mathrm{Hz})$ \\
\hline H2a-H3 & 5.5 & $\mathrm{H} 2 \mathrm{a}-\mathrm{C} 3$ & -0.7 \\
\hline $\mathrm{H} 2 \mathrm{~b}-\mathrm{H} 3$ & 9.2 & $\mathrm{H} 2 \mathrm{~b}-\mathrm{C} 3$ & -6.8 \\
\hline $\mathrm{H} 4-\mathrm{H} 5$ & 10.3 & H3-C2 & -2.6 \\
\hline $\mathrm{H} 6-\mathrm{H} 7 \mathrm{a}$ & 0.7 & $\mathrm{H} 4-\mathrm{C} 3$ & -1.5 \\
\hline H6-H7b & 10.9 & $\mathrm{H} 4-\mathrm{C} 5$ & -6.0 \\
\hline H7b-H8 & 9.9 & $\mathrm{H} 5-\mathrm{C} 4$ & -2.3 \\
\hline H13-H14 & 10.9 & H5-C18 & 1.7 \\
\hline \multirow[t]{16}{*}{ H14-H15 } & 10.4 & H6-C8 & 1.2 \\
\hline & & H7a-C6 & 4.7 \\
\hline & & $\mathrm{H} 7 \mathrm{~b}-\mathrm{C} 21$ & 1.6 \\
\hline & & $\mathrm{H} 8-\mathrm{C} 7$ & 2.9 \\
\hline & & $\mathrm{H} 13-\mathrm{C} 15$ & 1.5 \\
\hline & & $\mathrm{H} 13-\mathrm{C} 22$ & 7.8 \\
\hline & & $\mathrm{H} 13-\mathrm{C} 23$ & 3.6 \\
\hline & & $\mathrm{H} 14-\mathrm{C} 16$ & 2.8 \\
\hline & & $\mathrm{H} 15-\mathrm{C} 17$ & 3.8 \\
\hline & & $\mathrm{H} 15-\mathrm{C} 23$ & 1.8 \\
\hline & & H16a-C14 & 2.0 \\
\hline & & H16a-C15 & 5.7 \\
\hline & & $\mathrm{H} 16 \mathrm{~b}-\mathrm{C} 14$ & 0.1 \\
\hline & & H16b-C15 & 0.6 \\
\hline & & $\mathrm{H} 19 \mathrm{~b}-\mathrm{C} 7$ & 1.5 \\
\hline & & H14-C15 & 6.8 \\
\hline
\end{tabular}


Table 5.10: Experimental residual dipolar couplings of comp.540a in two alignment media.

\begin{tabular}{lrc}
\hline CH-vector & \multicolumn{2}{c}{${ }^{1} D_{\mathrm{CH}}(\mathrm{Hz})$} \\
& PH-gel & $(R)$-APhES-gel \\
\hline C2-H2a & -7.3 & 0.0 \\
C2-H2b & 9.3 & 16.8 \\
C3-H3 & -3.3 & 1.5 \\
C4-H4 & 0.0 & 2.2 \\
C5-H5 & 3.8 & 16.0 \\
C6-H6 & -8.6 & -24.0 \\
C7-H7a & -8.4 & -19.2 \\
C7-H7b & 15.5 & 17.4 \\
C8-H8 & 18.1 & 18.4 \\
C10-H10 & 12.1 & 18.1 \\
C11-H11 & 15.4 & 21.5 \\
C13-H13 & 18.2 & 21.4 \\
C14-H14 & 16.1 & 27.3 \\
C15-H15 & 16.3 & 22.6 \\
C18-H18 & 0.0 & -1.9 \\
C21-H21 & 0.0 & -5.3 \\
C22-H22 & -4.8 & -6.3 \\
C23-H23 & 1.9 & 4.7 \\
C1'-H1' & -5.2 & -8.2 \\
C2'-H2' & 8.4 & 18.4 \\
C3'-H3' & -6.6 & -9.1 \\
C4'H4' & -7.6 & -11.4 \\
C5'-H5' & -6.9 & -9.3 \\
C6'-H6' & -3.2 & -4.0 \\
\hline
\end{tabular}


Table 5.11: Proton-proton distances of comp.540a derived from a NOESY spectrum at $200 \mathrm{~ms}$ mixing time. All distances were referenced to $\mathrm{H7a} / \mathrm{H} 7 \mathrm{~b}$, considering the distance between two geminal protons to be $1.78 \AA$.

\begin{tabular}{llll}
\hline Atoms & Distance $(\AA)$ & Atoms & Distance $(\AA)$ \\
\hline H2a-H3 & 3.1 & H7a-H10 & 3.7 \\
H2a-H4 & 2.5 & H7b-H8 & 2.3 \\
H2a-H14 & 4.1 & H8-H10 & 4.0 \\
H2a-H15 & 4.2 & H8-H11 & 4.5 \\
H2b-H3 & 2.6 & H8-H19b & 2.7 \\
H2b-H4 & 3.3 & H8-H20 & 4.0 \\
H2b-H18 & 2.7 & H8-H21 & 3.0 \\
H3-H4 & 2.5 & H10-H21 & 2.9 \\
H3-H6 & 2.5 & H10-H22 & 2.2 \\
H3-H11 & 4.2 & H11-H13 & 2.3 \\
H4-H7b & 2.8 & H13-H14 & 2.8 \\
H4-H10 & 3.6 & H13-H15 & 3.2 \\
H4-H1' & 4.0 & H13-H23 & 2.9 \\
H4-H2' & 3.7 & H14-H15 & 2.8 \\
H5-H6 & 2.2 & H14-H16a & 3.3 \\
H5-H7a & 3.3 & H14-H16b & 2.4 \\
H5-H19a & 2.3 & H14-H23 & 2.4 \\
H5-H20 & 2.9 & H15-H16a & 2.9 \\
H5-H1' & 2.3 & H15-H16b & 2.7 \\
H5-H2' & 3.6 & H15-H23 & 2.9 \\
H6-H7b & 2.8 & H18-H1' & 2.7 \\
H6-H8 & 3.6 & H18-H2' & 2.8 \\
H7a-H8 & 2.6 & H18-H3' & 3.7 \\
\hline
\end{tabular}


method utilizes only the information of the distances derived from NMR, the conformation of the molecules cannot be defined in many cases due to insufficient experimental data and therefore the configuration cannot be determined unambiguously. Furthermore, for flexible molecules, NOE data are often not very sensitive to distinguish the correct and wrong conformations because of the minus $6^{\text {th }}$ power dependence. Until now, the fc-rDG/DDD method has been only applied to condensed ring systems such as tetrabomostyloguanidine [Grube and Köck, 2007], axinellamine A and 3,7-epi-massadine chloride [Köck et al., 2012], that are rigid in nature. To overcome the limitations of fc-rDG/DDD, in this study we decided to combine restrained MD simulation (r-MD) with the floating chirality method, in order to benefit from the precise conformational elucidation from the force field based MD simulation and the simultaneous determination of several configurations from the floating chirality method. Finally, we performed an RDC fitting on the obtained ensemble from the NOE- and $J$-restrained MD simulation to check the correctness of the structures and determine the correct configuration from the remaining possible configurations.

Restrained MD simulations of comp.540a were run for 40 ns at $298 \mathrm{~K}$ using GROMOS. The topology was manually parametrized based on the GROMOS 53A6 force field. Before starting the simulation, comp.540a was solvated in a DMSO solvent box with periodic boundary conditions. In our study the floating chirality method was achieved by excluding the improper dihedral energy term for each unknown stereocenter during the simulations. Experimental ${ }^{3} J_{\mathrm{HH}}$ and ${ }^{2,3} J_{\mathrm{CH}}$ coupling data indicate the existence of main dihedral conformations around C2-C3 and C14-C15 as shown in Figure 5.12 with Newman projections. Anti conformation of C1 and C4, and H14 and H15 were determined for the dihedral angles of C2-C3 and C14-C15, respectively. Together with the 46 NOE-derived proton-proton distances, they were used as restraints in the MD simulations.

Figure 5.13 and 5.14 show the evolution of improper dihedrals of the seven unknown stereocenters with simulation time. An improper dihedral between $0^{\circ}$ and $60^{\circ}$ corresponds to a different configuration than an improper dihedral between $300^{\circ}$ and $360^{\circ}$, indicating that the improper dihedral is a useful parameter for monitoring the transition of the configuration during the simulation time. In the restrained MD simulation force constants of $3000 \mathrm{kcal} /\left(\mathrm{mol} \cdot \mathrm{nm}^{2}\right)$ and $3000 \mathrm{kcal} /\left(\mathrm{mol} \cdot \mathrm{deg}^{2}\right)$ were applied for NOE and dihedral terms, respectively. As expected the inversion of the chirality for several stereocenters could be observed (Figure 5.13), which means that different stereoisomers could be generated during the simulations. For comparison, a MD simulation without NMR restraints was performed, showing a much 
Dihedral C2-C3

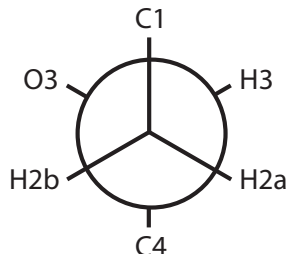

H2a: proR, H2b:proS, C3:S

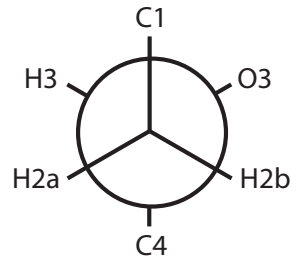

H2a: $\operatorname{pro} R, \mathrm{H} 2 \mathrm{~b}: \operatorname{proS}, \mathrm{C} 3: R$

\begin{tabular}{lll}
\hline${ }^{3}(\mathrm{H} 2 \mathrm{a}-\mathrm{H} 3)$ & $5.5 \mathrm{~Hz}$ & extracted from P.E. COSY spectrum \\
${ }^{3} \mathrm{~J}(\mathrm{H} 2 \mathrm{~b}-\mathrm{H} 3)$ & $9.2 \mathrm{~Hz}$ & extracted from P. E. COSY spectrum \\
${ }^{2} \mathrm{~J}(\mathrm{H} 2 \mathrm{a}-\mathrm{C} 3)$ & $-0.7 \mathrm{~Hz}$ & extracted from $\left[{ }^{1} \mathrm{H}-{ }^{13} \mathrm{C}\right]-\mathrm{HECADE}$ spectrum \\
${ }^{2} \mathrm{~J}(\mathrm{H} 2 \mathrm{~b}-\mathrm{C} 3)$ & $-6.8 \mathrm{~Hz}$ & extracted from $\left[{ }^{1} \mathrm{H}-{ }^{13} \mathrm{C}\right]-\mathrm{HECADE}$ spectrum \\
\hline
\end{tabular}

Dihedral C14-C15

$\mathrm{C} 14: R, \mathrm{C} 15: R$

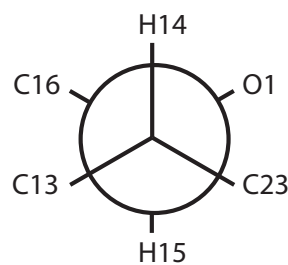

or

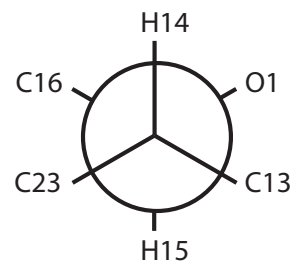

C14:S, C15:S<smiles>CC12CC([18OH])C3CC(C)(CC(C1)C3(Cl)Cl)C2(Cl)Cl</smiles>

or

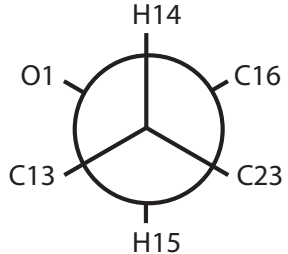

\begin{tabular}{lll}
\hline${ }^{3}(\mathrm{H} 14-\mathrm{H} 15)$ & $10.4 \mathrm{~Hz}$ & extracted from COSY spectrum \\
${ }^{3}(\mathrm{H} 14-\mathrm{H} 16)$ & $2.8 \mathrm{~Hz}$ & extracted from $\left[{ }^{1} \mathrm{H}-{ }^{13} \mathrm{C}\right]-\mathrm{HECADE}$ spectrum \\
& & \\
${ }^{3} \mathrm{~J}(\mathrm{H} 15-\mathrm{C} 23)$ & $1.8 \mathrm{~Hz}$ & extracted from $\left[{ }^{1} \mathrm{H}-{ }^{13} \mathrm{C}\right]-\mathrm{HECADE}$ spectrum
\end{tabular}

Figure 5.12: Newman projections of possible conformers for dihedral C2-C3 and C14$\mathrm{C} 15$, respectively. $J$-coupling analysis suggests that $\mathrm{C} 1$ and $\mathrm{C} 4$ are anti to each other in the dihedral of $\mathrm{C} 2-\mathrm{C} 3$, and $\mathrm{H} 14$ and $\mathrm{H} 15$ are also anti to each other in the dihedral of C14-C15.

more stable trend to maintain the original configuration (Figure 5.14). This result clearly implies the importance of the NOE and dihedral energy terms in changing 
the chirality and the development of the correct relative configuration during the simulation time. In order to understand the influence of the starting configuration and to study the robustness of the method, three independent restrained MD simulations were performed with different starting configurations (first: SSSRSSR, second: $R R S R S R S$, third: $S S R S S R S$ ), that have been chosen randomly. During these simulations, only stereocenters of the macrolide were left floating, whereas those of the sugar moiety were fixed. Here we assumed that the rhamnose has a D form and the $\alpha$-configuration of the sugar was established from the evidence that the experimental ${ }^{3} J$-couplings of H1-C3 and H1-C5 are both 7.2 Hz.

While analyzing the trajectories we found that simulations I-III yield different configurations with lowest dihedral and NOE deviation energy (Table 5.12). Nevertheless, when $10 \%, 15 \%$ or $20 \%$ of the snapshots with lowest deviation energy were taken into account, the snapshots from simulation I and II of the configurations $R S S S R S R$ and $R S R S R S R$ were mainly populated, whereas the snapshots of the enantiomeric configurations $S R R R S R S$ and $S R S R S R S$ were found to have the lowest deviation energy in simulation III (Figure 5.15 and Table A.17). Since the population of these configurations with lowest deviation energy reflects how well the restraints are fulfilled, it is evident that using our proposed method the extensive possibilities of the relative configurations could be significantly reduced. Furthermore, due to the fact that the highly populated relative configurations are identical in simulations I-III, we can conclude that the final result does not depend on the starting configuration. However, the presence of the enantiomeric configurations in simulation III suggests that only the relative configuration of the macrolide itself can be determined in our case, but not the relative configuration between the macrolide and the sugar moiety. This is not entirely surprising, considering that the rotation around the glycosidic dihedral angles $\phi$ and $\psi$ using conventional MD is on the microsecond timescale [Hansen and Hünenberger, 2011], however the performed MD simulations are on the nanosecond timescale. Therefore the correct orientation and relative configuration between the macrolide ring and the sugar was difficult to be assessed using our method.

In order to determine the correct configuration of the macrolide lactone ring from the remaining possible configurations, a cross-validation against the RDC data was performed. For each of the four configurations with lowest deviations obtained from simulations I and II (RSSSRSR, RSRSRSR, RSRSSSR, SSRSRSR), a $10 \mathrm{~ns}$ free MD simulation was carried out without NMR restraints. This additional step enables sampling the conformational space more extensively and removes distortions 


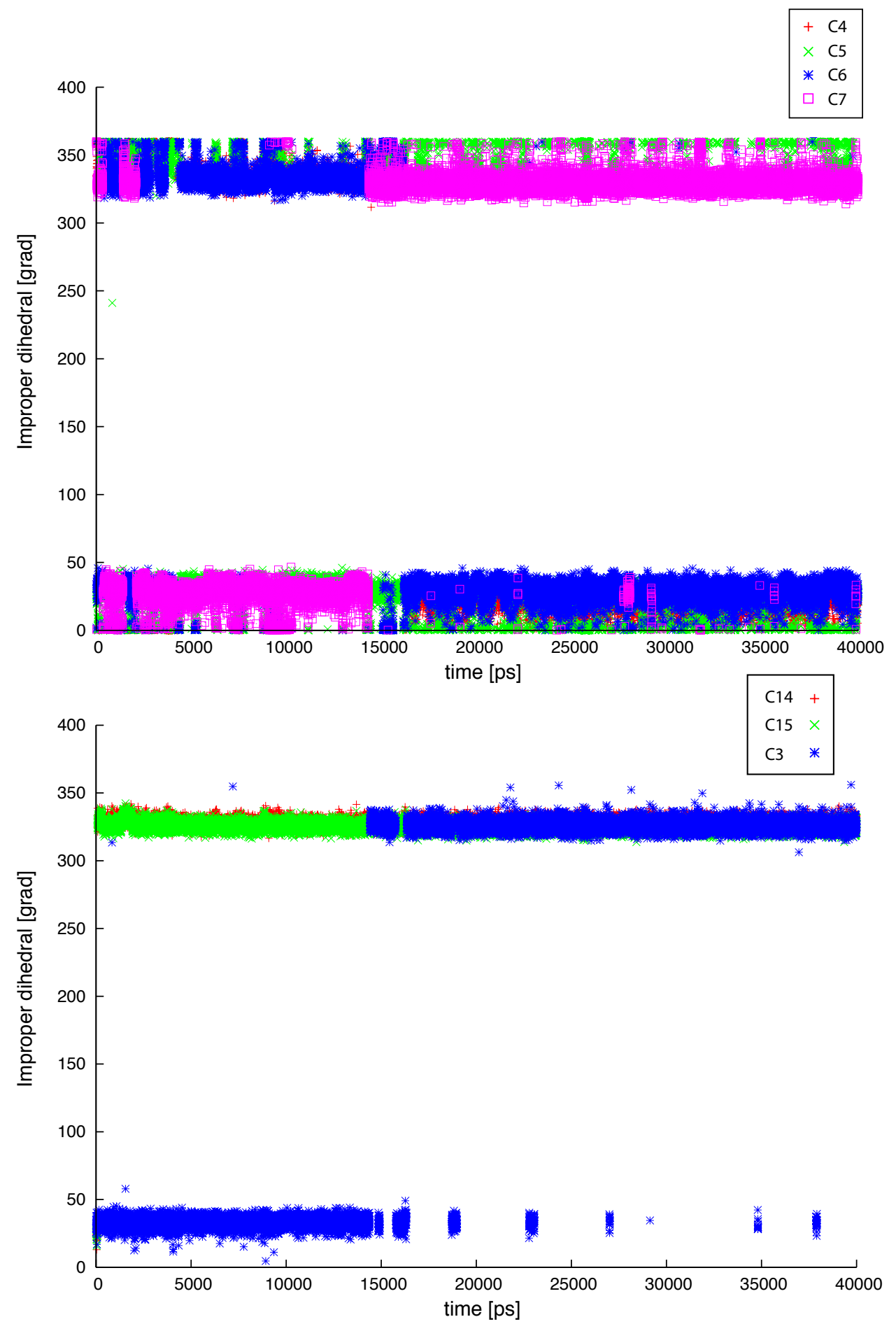

Figure 5.13: Improper dihedral angles of the seven unknown centers (C3, C4, C5, C6, C7, $\mathrm{C} 14, \mathrm{C} 15)$ as a function of time for a restrained MD simulation with the floating chirality method.

of the structure resulting from the experimental errors of $J$-couplings and NOEs. For each calculated trajectory, the snapshots were clustered based on the similarity of the conformations and the four most populated main clusters were selected as 


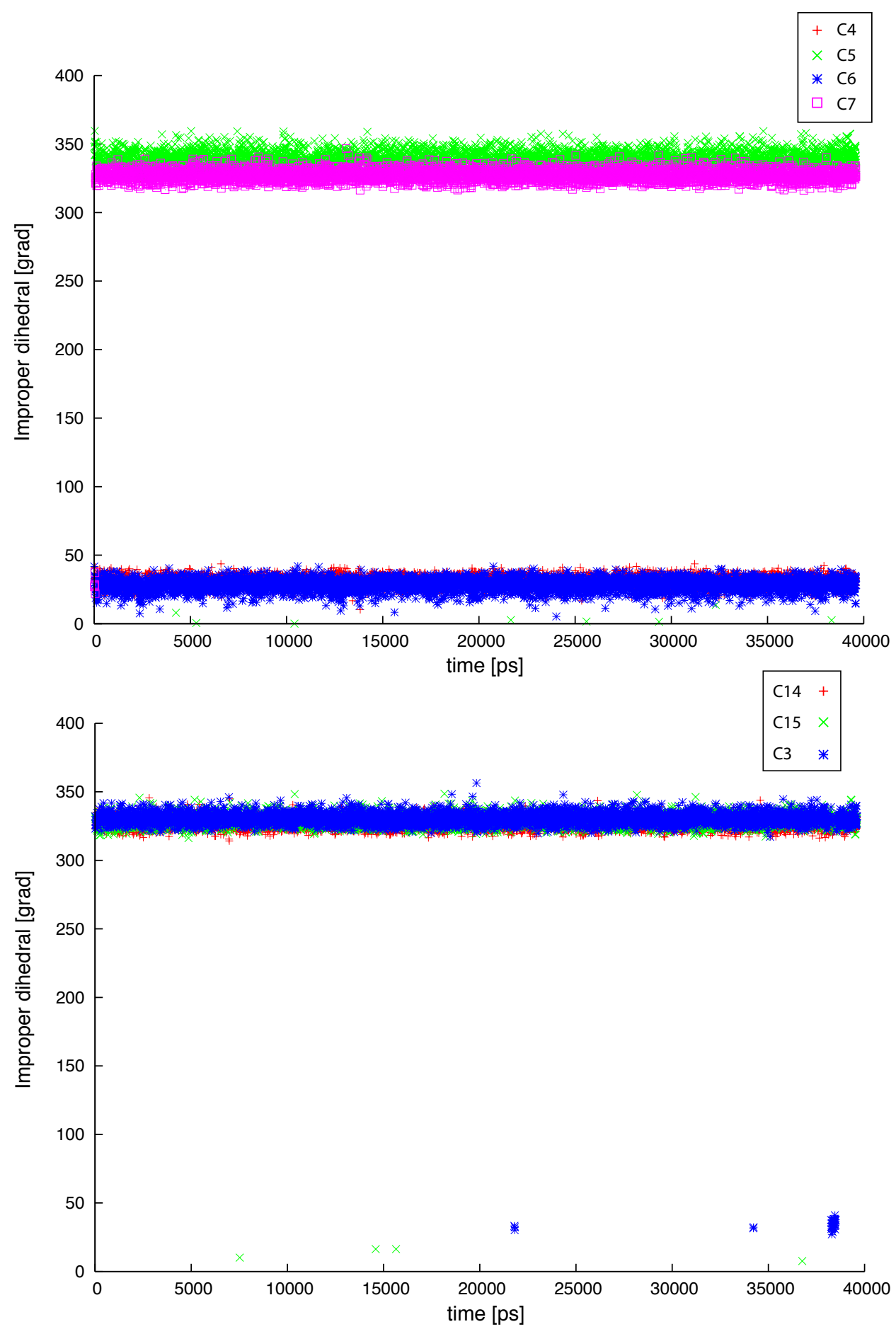

Figure 5.14: Improper dihedral angles of the seven unknown centers (C3, C4, C5, C6, $\mathrm{C} 7, \mathrm{C} 14, \mathrm{C} 15)$ as a function of time for a free MD simulation with the floating chirality method.

the main conformations. The ensemble of the main conformations were then fitted against two sets of RDC data from the macrolide moiety (Table 5.10), and at the same time the populations were calculated based on the experimental RDCs (Ta- 
Table 5.12: The configurations with the lowest NOE and dihedral deviation energy generated in the restrained MD simulations with the floating chirality method.

\begin{tabular}{llll}
\hline & $\begin{array}{l}\text { Configuration with } \\
\text { lowest NOE and di- } \\
\text { hedral deviation }\end{array}$ & $\begin{array}{l}\text { Dihedral deviation } \\
\left(\mathrm{kcal} \cdot \mathrm{mol}^{-1}\right)\end{array}$ & $\begin{array}{l}\text { NOE deviation } \\
\left(\mathrm{kcal} \cdot \mathrm{mol}^{-1}\right)\end{array}$ \\
\hline Simulation I & $R S S S R S R$ & 8.71 & 94.25 \\
Simulation II & $R S R S R S R$ & 7.68 & 91.84 \\
Simulation III & $S R R R S R S$ & 6.48 & 78.10 \\
\hline
\end{tabular}

ble 5.13). For this purpose, we used the SVD method to determine the alignment tensor and population simultaneously, as implemented in an in-house version of the program MSpin. Similar to the previous study of an Michael addition product (see Chapter 4.1), the single tensor assumption was used due to the high similarity of the main conformations of the macrolides (Figure 5.16). Since only the RDCs of the macrolide were included in the calculations, the relative orientation of conformers in the ensemble is determined by superimposing each to the mean structure of the macrolide ring.

The result of the RDC analysis is given in Table 5.13 and Figure A.14, which shows that two configurations fit well with low $Q$ factors: $R S S S R S R$ (PH-gel: $Q=0.085$, APhEs-gel: $Q=0.113), R S R S R S R$ (PH-gel: $Q=0.102$, APhEs-gel: $Q=0.202)$. Nevertheless, the lowest $Q$ factor was obtained for the configuration $R S S S R S R$ for both RDC data sets, and the $Q$ factor difference in the APhEs-gel data is significant. The 5D angle (Equation 2.39) between the two calculated tensors is $63.8^{\circ}$, indicating that the two RDC data sets are independent from each other. At this step we can conclude that the relative configuration of the stereocenters in the macrolide ring can be determined as $\mathrm{C} 3: R, \mathrm{C} 4: S, \mathrm{C} 5: S, \mathrm{C} 6: S, \mathrm{C} 7: R, \mathrm{C} 14: S$, $\mathrm{C} 15: R$, or its enantiomer. Another evidence of $R S R S R S R$ as the wrong configuration comes from the $J$-coupling analysis of C5-C6, shown in Figure A.15, as the two configurations that need to be distinguished ( $R S R S R S R$ and $R S R S R S R$ ) only differ in the relative configuration of the stereocenters C5 and C6. 


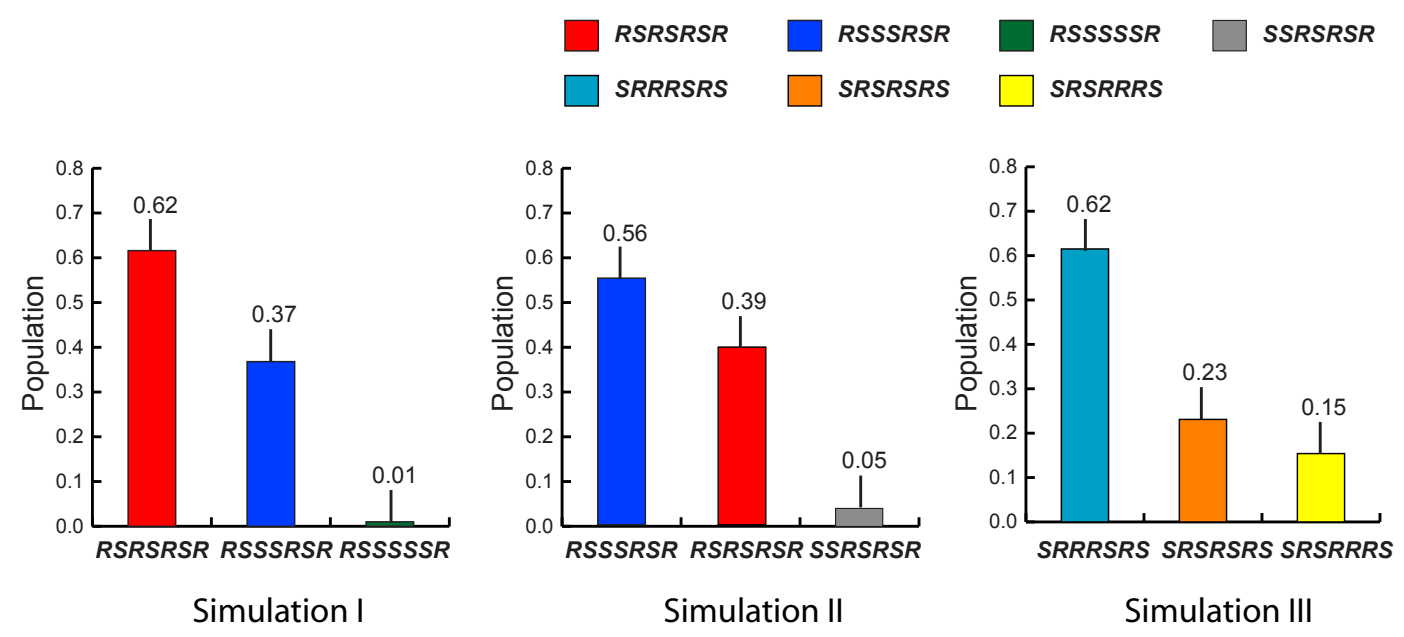

Figure 5.15: The populations of the configurations determined from the snapshots of the restrained MD simulations with $20 \%$ lowest deviation energy. The three independent simulations I, II and III were performed with the floating chirality method and started with different initial configurations.

\subsubsection{Elucidation of the relative configuration between the macrolide ring and the sugar}

After determining the relative configuration in the macrolide backbone, we turned our focus on the establishment of the relative configuration between the macrocyclic lactone ring and the sugar. If the configuration of the lactone ring is fixed to $R S S S R S R$, then the sugar attached at C5 can be either $\alpha$-D-rhamnose or $\alpha$ L-rhamnose. The determination of the relative configuration of these two moieties requires the simultaneous elucidation of the conformation. However, as already mentioned before, the rotation around the glycosidic dihedral angles $\phi$ and $\psi$ in solution is on the microsecond time scale, therefore it is very difficult to sample the conformational space of the carbohydrates using conventional MD-simulations within a reasonable simulation time. This problem has been also observed in our $10 \mathrm{~ns}$ free MD simulation, as the dihedral angles $\phi$ and $\psi$ do not change significantly from the original values during the simulation. To overcome this limitation, we have used here another approach, which is to rotate the $\phi$ and $\psi$ dihedrals manually from $-60^{\circ}$ to $300^{\circ}$ in steps of $120^{\circ}$, followed by an energy minimization. In other words, $+\mathrm{g}$, $-\mathrm{g}$ and $\mathrm{t}$ conformations for each dihedral were generated, resulting in 9 different conformers in total for each possible relative configuration. After discarding high energy structures, 8 and 7 conformers remained within an energy difference of 10 $\mathrm{kcal} \cdot \mathrm{mol}^{-1}$ for the configuration with $\alpha$-L-rhamnose and $\alpha$-D-rhamnose, respectively (Table A.18). In Figure A.16, 8 conformers with different sugar orientations are 
Table 5.13: The $Q$ factors of the RDC fitting and populations of the main conformations determined by the RDC data. The configurations in the table were selected based on low $J$-coupling and NOE deviation energy in the restrained MD simulations with the floating chirality method. The RDC fitting was performed using a structure ensemble assuming a uniform alignment tensor for different conformations within the ensemble.

\begin{tabular}{llllll}
\hline $\begin{array}{l}\text { Alignment } \\
\text { medium }\end{array}$ & Configuration & \multicolumn{3}{c}{ Population } & $Q$ factor \\
& & Conformer 1 & Conformer 2 & Conformer 3 \\
\hline PH-gel & RSSSRSR & $15 \%$ & $49 \%$ & $36 \%$ & 0.085 \\
& $R S R S R S R$ & $64 \%$ & $36 \%$ & - & 0.102 \\
& $R S S S S S R$ & $100 \%$ & - & - & 0.412 \\
& $S S R S R S R$ & $75 \%$ & $15 \%$ & $10 \%$ & 0.393 \\
\hline \multirow{2}{*}{ APhEs-gel } & $R S S S R S R$ & $39 \%$ & $22 \%$ & $39 \%$ & 0.113 \\
& $R S R S R S R$ & $61 \%$ & $38 \%$ & - & 0.202 \\
& $R S S S S S R$ & $59 \%$ & $40 \%$ & - & 0.336 \\
& $S S R S R S R$ & $50 \%$ & $29 \%$ & $21 \%$ & 0.574 \\
\hline
\end{tabular}

depicted for the configuration with $\alpha$-L-rhamnose.

To determine the correct orientation between the sugar and the lactone ring, SVD fitting of the RDC data was performed on the individual conformers of the configurations with $\alpha$-D-rhamnose and $\alpha$-L-rhamnose, respectively (Table A.18). The conformers with the lowest $Q$ factor were selected for the two possible configurations. Results summarized in Table 5.14 suggest that the $\alpha$-L-rhamnose has a better agreement with the RDC data in both alignment media. Additionally, seven specific NOEs between macrolide and sugar moiety could be identified and quantitatively analyzed. No significant NOE violation was observed for the $\alpha$-L-rhamnose configurations, whereas three distances between the sugar protons and lactone protons are incompatible with the experimental NOEs for the $\alpha$-D-rhamnose configurations. In conclusion, both NOE and RDC analysis suggest the configuration of the macrolide to be $R S S S R S R$ and of the sugar to be $\alpha$-L-rhamnose or of the macrolide to be $S R R R S R S$ and of the sugar to be $\alpha$-D-rhamnose, since the enantiomeric pairs cannot be distinguished by the present NMR data. The dihedral angles $\phi$ and $\psi$ of the determined conformers are $26.80^{\circ}$ and $-88.26^{\circ}$, respectively.

In the mean time, the crystal structure of comp.540a has been solved independently by our collaborators from the Scripps Research Institute. A comparison of 


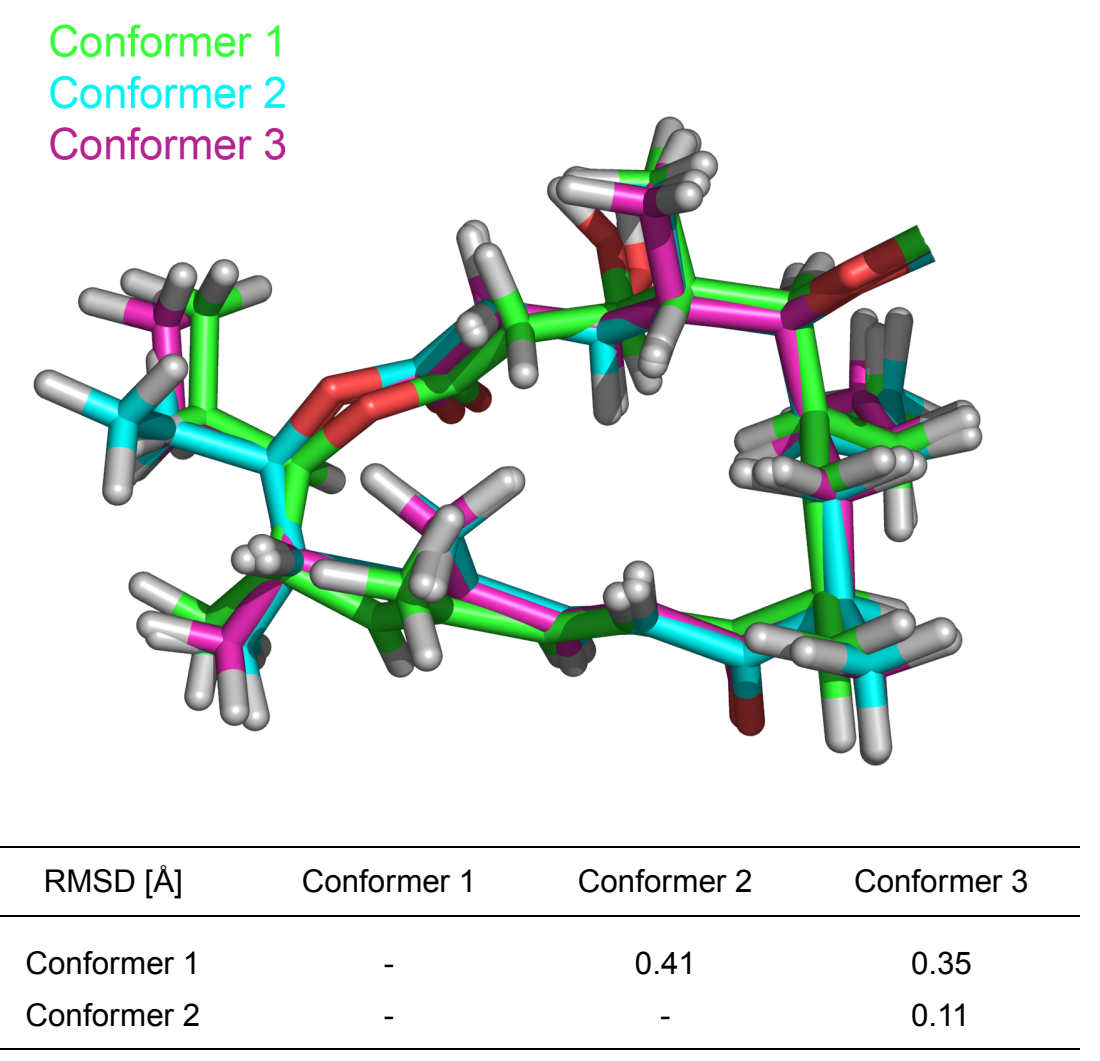

Figure 5.16: Main conformations of the best fitting configuration RSSSRSR superimposed on the heavy atoms of the macrolide ring system. The RMSD was calculated based on the heavy atoms of the macrolide ring system.

the NMR and crystal structures, as shown in Figure 5.17, highlights the strong similarity of these two structures, for which the calculated RMSD of all heavy atoms is $0.49 \AA$. In conclusion, NMR and X-ray crystallography, independently led to the same relative configuration and surprisingly also to a similar conformation of the macrocyclic lactone ring, as well as to the same relative orientation between the lactone ring and the sugar.

\subsubsection{Conclusion}

Restrained MD simulations with the floating chirality method combined with NMR data including RDC obtained in two different gel-based alignment media enabled the simultaneous determination of the relative configuration and conformation of comp.540a, a novel macrolide that has been recently isolated from the marine actinobacteria Solwaraspora. Three independent MD simulations that were performed with different starting configurations, resulted in the same relative configurations $R S S S R S R$ or $R S R S R S R$ with the lowest deviation energy, demonstrating the ro- 
Table 5.14: The $Q$ factor and deviation of 7 specific NOEs in $\AA^{2}$ for two different sugar configurations.

\begin{tabular}{llll}
\hline & & $\alpha$-D-rhamnose & $\alpha$-L-rhamnose \\
\hline$Q$ factor & PH-gel & 0.24 & 0.13 \\
& $(R)$-APhES-gel & 0.31 & 0.23 \\
\hline NOE deviation & H18'-H1 & 0.08 & 0.09 \\
$\left(\AA^{2}\right)$ & H18'-H2 & 3.81 & 0.15 \\
& H18'-H3 & 2.04 & 0.45 \\
& H1'-H5 & 0.02 & 0.01 \\
& H2'-H4 & 2.27 & 0.05 \\
& H2'-H5 & 0.01 & 0.08 \\
& H1'-H4 & 0.04 & 0.02 \\
\hline
\end{tabular}

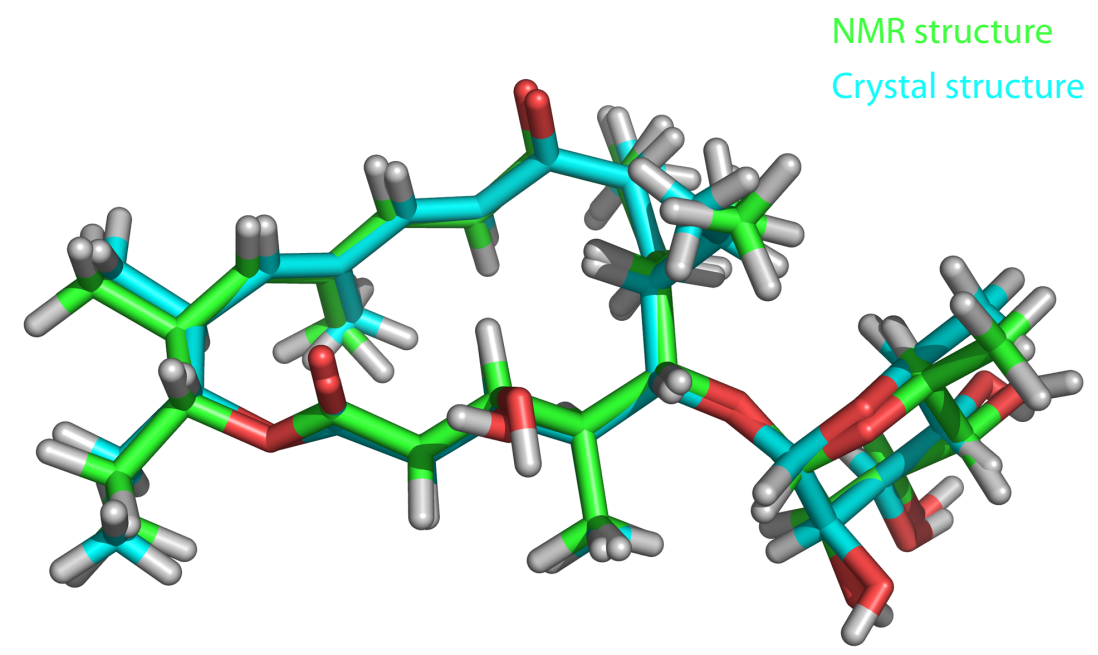

Figure 5.17: Comparison of the structures determined by NMR and X-ray.

bustness of the applied method. SVD fitting of the RDC data was utilized to identify the correct relative configuration from the remaining stereochemical possibilities that could not be distinguished on the basis of the NOE and $J$-restrained MD simulations alone. Furthermore, the usage of the complete RDC data sets allowed the elucidation of the orientation between macrocyclic lactone ring and the sugar. Using our proposed method, the relative configuration of the macrocyclic lactone ring was determined as $R S S S R S R S$ and the sugar as $\alpha$-L-rhamnose, or its enantiomeric pair, and the conformation is shown in Figure 5.17.

In the same time, the crystal structure of comp.540a was solved, approving the 
determined relative configuration based on the NMR data. Comparison of the NMR and crystal structures revealed a nearly identical backbone conformation, indicating the high precision of the NMR determined structure and the successful application of our proposed method. Different from the previously introduced approaches, such as the "fc-rDG/DDD" method [Grube and Köck, 2007,Köck et al., 2012] that only relies on NOE derived distance information, three different sources of experimental data ( $J$-couplings, NOEs and RDCs) from isotropic and anisotropic NMR experiments were employed in our study, increasing significantly the reliability of the obtained results. Future investigations will focus on the direct incorporation of the RDC data in the MD simulations, rather than performing cross-validation of the RDC data against the $J$ - and NOE-determined ensembles.

Tylosin, whose absolute configuration was determined by X-ray crystallography [Omura et al., 1980], exhibits the same relative configuration $R S S S R S R$ of the macrocyclic lactone ring as comp.540a. If we assume that both natural products share the same absolute configuration, then the sugar attached at C5 of comp.540a should be $\alpha$-L-rhamnose, which belongs to one of few natural products that occur only in the L-form naturally [Wollrab, 2009]. 


\section{Chapter 6}

\section{Stereochemical elucidation of a molecule of class B2}

\subsection{Determination of the relative configuration of fibrosterol sulfate A}

\subsubsection{Introduction}

In this chapter, the molecule of interest is fibrosterol sulfate A [Whitson et al., 2009], a polysulfated sterol dimer isolated from the Philippine sponge Lissodendoryx (Acanthodoryx) fibrosa. It exhibits micromolar activity $\left(\mathrm{IC}_{50}=16.4 \mu \mathrm{M}\right)$ as an inhibitor of protein kinase $\mathrm{C} \zeta(\mathrm{PKC} \zeta)$, a protein involved in several types of cancer [Guo et al., 2009, Zhao et al., 2007, Cohen et al., 2006, Sun et al., 2005, Mustafi et al., 2006], obesity [Sajan et al., 2009], and osteoarthritis [Zhao et al., 2007].

The constitution of the compound was established on the basis of 2D NMR correlations as well as high-resolution electron spray ionization mass spectra (HRESIMS) analysis. The structure of this compound (Figure 6.1) consists of two steroid parts whose relative and absolute configuration was determined by comparison of the chemical shifts of steroids of known absolute configuration and biosynthetic considerations [Whitson et al., 2009]. The linker between the two steroid parts contains five additional stereocenters, of which C20 and C20' were assigned again by biosynthetic considerations. As described later, the configuration of these two stereocenters is corroborated by our RDC analysis. Yet, three stereocenters in the linker, C22, C25, and C24' (Figure 6.1), had not been assigned before. Long-range information is required to determine the configuration of these three unassigned stereocenters, which led us to use an RDC-based analysis. 
Due to the fact that conformational movement in the linker part can dramatically affect the global molecular shape, RDC analysis becomes problematic as orientational ordering is not independent of the conformation. Early approaches to the conformational distribution problem were the additive potential or maximum entropy methodologies [Catalano et al., 1991, Emsley et al., 1982, Stevensson et al., 2003, Thaning et al., 2008]. However, most of the recent applications to flexible molecules make use of the so-called "single-tensor" approach [Schuetz et al., 2007, Trigo-Mouriño et al., 2010, Sun et al., 2011a], which assumes the orientational ordering of a molecule is largely independent of its conformation. This assumption generally holds if the overall molecular shape is similar between different conformations as judged by structural alignments minimizing RMSD of atom positions. If the shapes of the various conformations are very different, for each conformation a different alignment tensor needs to be used. In this "multiple-tensor" approach, the number of required experimental anisotropic parameters increases in most cases beyond experimental measurement possibilities, and has so far been used only in simple systems [Thiele et al., 2009]. However, one can predict based on known alignment mechanisms the relative sizes of alignment tensors and their orientations for each conformation in the ensemble. Then the experimental anisotropic parameters can be cross-validated against these predictions. Indeed, this approach has been successfully applied for the cross-validation of ensembles of intrinsically unfolded proteins against anisotropic parameters [Bernadó et al., 2005a, Jha et al., 2005, Bernadó et al., 2005b, Mukrasch et al., 2007]. However it has not been applied successfully so far to small molecules in organic solvents.

Fibrosterol sulfate A clearly belongs to the molecule class B2 (Figure 1.2), as the two stereochemical domains 1 (C22) and 2 (C24' and C25) are separated by a flexible linker. Since this class of molecules has not been studied so far using RDC-based techniques, it was necessary to explore different methodologies for the stereochemical elucidation as illustrated above. In this study, a multiple alignment tensors analysis complemented by explicit-solvent molecular dynamics simulations was utilized for the relative stereochemical elucidation, in which the main conformations and the populations were determined from the MD simulations. Furthermore, we applied the single-tensor approach and explored the limitation of this much simpler method. 


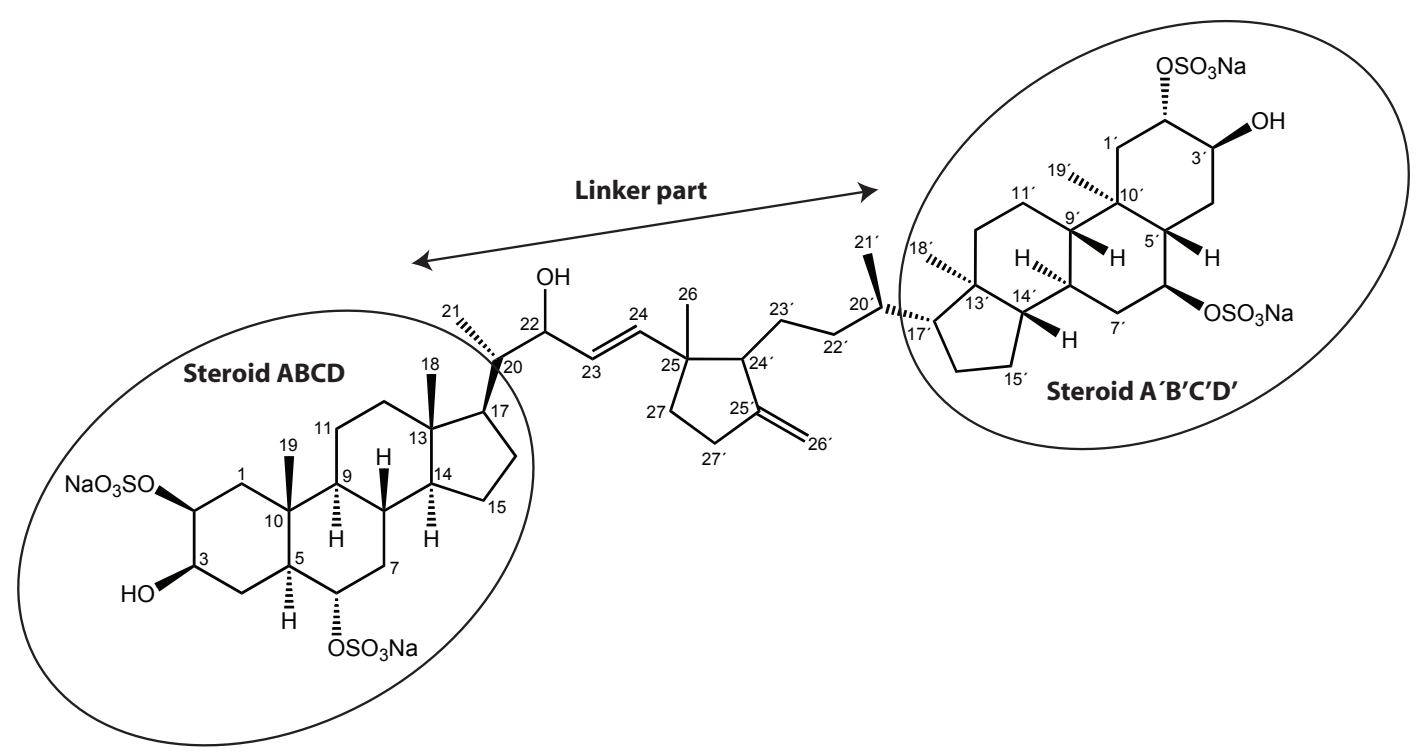

Figure 6.1: Structure of fibrosterol sulfate A with atom numbering. For convenience, the structure has been divided into three regions: steroid ABCD, linker part, and steroid A'B'C'D'. Determination of the configurations of positions 22,25 , and 24 ' is the main focus of this study.

\subsubsection{Elucidation of the relative configuration and conforma- tion by multiple-tensor analysis}

\section{NMR resonance assignment}

Fibrosterol sulfate A has a pseudosymmetric structure with two disulfated steroidal tetracyclic moieties (ABCD and A'B'C'D', Figure 6.1) joined by a flexible linker containing a cyclopentyl unit. Complete chemical shift assignments for fibrosterol sulfate A in DMSO- $d_{6}$ were determined using a combination of homo- and heteronuclear correlation experiments, such as $1 \mathrm{D}^{1} \mathrm{H}$, COSY, NOESY, TOCSY, $\left[{ }^{1} \mathrm{H},{ }^{13} \mathrm{C}\right]-$ HSQC, and $\left[{ }^{1} \mathrm{H},{ }^{13} \mathrm{C}\right]-\mathrm{HMBC}$ and are listed in Table 6.1. All NMR spectra were recorded at $25^{\circ} \mathrm{C}$.

Table 6.1: Carbon and proton chemical shifts of fibrosterol sulfate A in DMSO- $d_{6}$ at $298 \mathrm{~K}$.

\begin{tabular}{llll}
\hline Carbon & Chemical shift $(\mathrm{ppm})$ & Proton & Chemical shift $(\mathrm{ppm})$ \\
\hline C1 & 42.44 & H1ax & 1.033 \\
& & H1eq & 2.034 \\
C2 & 75.67 & H2 & 4.390 \\
\hline
\end{tabular}


Table 6.1: Carbon and proton chemical shifts of fibrosterol sulfate A in DMSO- $d_{6}$ at $298 \mathrm{~K}$.

\begin{tabular}{|c|c|c|c|}
\hline Carbon & Chemical shift (ppm) & Proton & Chemical shift (ppm) \\
\hline \multirow[t]{2}{*}{ C3 } & 71.33 & H3 & 3.313 \\
\hline & & $\mathrm{OH} 3$ & 4.673 \\
\hline \multirow[t]{2}{*}{$\mathrm{C} 4$} & 27.75 & $\mathrm{H} 4 \mathrm{ax}$ & 1.286 \\
\hline & & H4eq & 1.855 \\
\hline C5 & 50.57 & $\mathrm{H} 5$ & 0.924 \\
\hline C6 & 74.05 & $\mathrm{H} 6$ & 3.831 \\
\hline \multirow[t]{2}{*}{$\mathrm{C} 7$} & 39.49 & H7ax & 0.715 \\
\hline & & H7eq & 2.145 \\
\hline $\mathrm{C} 8$ & 33.92 & H8 & 1.330 \\
\hline C9 & 54.46 & H9 & 0.540 \\
\hline $\mathrm{C} 10$ & 36.73 & & \\
\hline \multirow[t]{2}{*}{$\mathrm{C} 11$} & 11.27 & H11ax & 1.212 \\
\hline & & H11eq & 1.400 \\
\hline \multirow[t]{2}{*}{$\mathrm{C} 12$} & 39.95 & H12ax & 1.033 \\
\hline & & H12eq & 1.890 \\
\hline $\mathrm{C} 13$ & 42.81 & & \\
\hline $\mathrm{C} 14$ & 55.98 & H14 & 0.933 \\
\hline \multirow[t]{2}{*}{$\mathrm{C} 15$} & 24.56 & H15ax & 1.024 \\
\hline & & H15eq & 1.459 \\
\hline \multirow[t]{2}{*}{ C16 } & 27.48 & H16a & 1.248 \\
\hline & & $\mathrm{H} 16 \mathrm{~b}$ & 1.699 \\
\hline $\mathrm{C} 17$ & 53.29 & H17 & 0.974 \\
\hline C18 & 12.49 & H18 & 0.620 \\
\hline C19 & 15.61 & H19 & 0.878 \\
\hline $\mathrm{C} 20$ & 42.48 & H20 & 1.545 \\
\hline $\mathrm{C} 21$ & 12.95 & H21 & 0.824 \\
\hline \multirow[t]{2}{*}{$\mathrm{C} 22$} & 73.35 & H22 & 3.895 \\
\hline & & $\mathrm{OH} 22$ & 4.441 \\
\hline $\mathrm{C} 23$ & 127.03 & H23 & 5.237 \\
\hline $\mathrm{C} 24$ & 135.06 & H24 & 5.422 \\
\hline $\mathrm{C} 25$ & 46.85 & & \\
\hline $\mathrm{C} 26$ & 25.14 & H26 & 1.072 \\
\hline \multirow[t]{2}{*}{$\mathrm{C} 27$} & 37.00 & $\mathrm{H} 27 \mathrm{a}$ & 1.645 \\
\hline & & $\mathrm{H} 27 \mathrm{~b}$ & 1.496 \\
\hline
\end{tabular}


Table 6.1: Carbon and proton chemical shifts of fibrosterol sulfate A in DMSO- $d_{6}$ at $298 \mathrm{~K}$.

\begin{tabular}{|c|c|c|c|}
\hline Carbon & Chemical shift (ppm) & Proton & Chemical shift (ppm) \\
\hline \multirow[t]{2}{*}{$\mathrm{C} 1{ }^{\prime}$} & 37.90 & H1'ax & 1.235 \\
\hline & & H1'eq & 1.771 \\
\hline $\mathrm{C} 2{ }^{\prime}$ & 75.27 & $\mathrm{H} 2^{\prime}$ & 4.133 \\
\hline \multirow[t]{2}{*}{ C3' } & 66.60 & H3' & 3.806 \\
\hline & & OH3' & 4.537 \\
\hline \multirow[t]{2}{*}{ C4' } & 25.89 & H4'ax & 1.374 \\
\hline & & H4'eq & 1.769 \\
\hline $\mathrm{C} 5$ & 43.61 & $\mathrm{H} 5$ & 1.442 \\
\hline C6 & 74.76 & $\mathrm{H} 6$ & 3.833 \\
\hline \multirow[t]{2}{*}{$\mathrm{C} 7$} & 39.55 & H7'ax & 0.786 \\
\hline & & H7'eq & 2.150 \\
\hline C8 & 33.81 & $\mathrm{H} 8$ & 1.336 \\
\hline C9' & 54.61 & H9' & 0.589 \\
\hline C10' & 36.79 & & \\
\hline \multirow[t]{2}{*}{ C11' } & 20.85 & H11'ax & 1.176 \\
\hline & & H11'eq & 1.439 \\
\hline \multirow[t]{2}{*}{ C12' } & 40.06 & H12'ax & 1.083 \\
\hline & & H12'eq & 1.921 \\
\hline C13' & 42.66 & & \\
\hline C14' & 56.38 & H14' & 1.019 \\
\hline \multirow[t]{2}{*}{ C15 } & 34.36 & H15'ax & 1.019 \\
\hline & & H15’eq & 1.515 \\
\hline \multirow[t]{2}{*}{ C16 } & 28.31 & H16’a & 1.222 \\
\hline & & $\mathrm{H} 16$ 'b & 1.798 \\
\hline C17' & 56.14 & H17' & 1.087 \\
\hline C18' & 12.64 & H18' & 0.628 \\
\hline C19' & 15.06 & H19' & 0.856 \\
\hline C20' & 36.62 & H20' & 1.328 \\
\hline $\mathrm{C} 21^{\prime}$ & 19.26 & $\mathrm{H} 21{ }^{\prime}$ & 0.920 \\
\hline \multirow[t]{2}{*}{ C22' } & 35.65 & H22'a & 1.585 \\
\hline & & $\mathrm{H} 22^{\prime} \mathrm{b}$ & 0.929 \\
\hline \multirow[t]{2}{*}{ C23' } & 25.50 & H23'a & 1.017 \\
\hline & & $\mathrm{H} 23^{\prime} \mathrm{b}$ & 1.385 \\
\hline C25' & 150.99 & & \\
\hline
\end{tabular}


Table 6.1: Carbon and proton chemical shifts of fibrosterol sulfate A in DMSO- $d_{6}$ at $298 \mathrm{~K}$.

\begin{tabular}{lcll}
\hline Carbon & Chemical shift $(\mathrm{ppm})$ & Proton & Chemical shift $(\mathrm{ppm})$ \\
\hline C24' & 55.90 & H24' & 1.791 \\
C26' & 105.18 & H26'a & 4.755 \\
& & H26'b & 4.811 \\
C27' & \multirow{2}{*}{29.29} & H27'a & 2.349 \\
& & H27'b & 2.349 \\
\hline
\end{tabular}

\section{Measurements of scalar and residual dipolar couplings}

The ${ }^{3} J_{\mathrm{HH}}$ couplings and ${ }^{3} J_{\mathrm{CH}}$ couplings (Table 6.2) were extracted from P.E.COSY and HSQMBC spectra, respectively. In the HSQMBC sequence, the delay for the evolution of ${ }^{n} J_{\mathrm{CH}}$ couplings was set to $125 \mathrm{~ms}$.

For the RDC measurements $650 \mu \mathrm{L}$ DMSO- $d_{6}$ was added to a $5 \mathrm{~mm}$ standard NMR tube together with a dried PAN polymer stick, which was prepared in the group of Prof. Burkhard Luy (Institut für Organische Chemie, Karlsruher Institut für Technologie, Karlsruhe, Germany). The swelling of the gel was monitored by observing the ${ }^{2} \mathrm{D}$ quadrupolar splitting of the solvent signal. After one month, the gel was equilibrated, giving a final splitting of $18.1 \mathrm{~Hz}$. Fibrosterol sulfate A (5 mg) was dissolved in $50 \mu \mathrm{L}$ of DMSO- $d_{6}$ and added on top of the gel which was further equilibrated during 3 weeks at room temperature. $44{ }^{1} D_{\mathrm{CH}}$ RDCs were extracted by measuring the splitting difference between isotropic and anisotropic signals from $\left[{ }^{1} \mathrm{H},{ }^{13} \mathrm{C}\right]$-CLIP-HSQC spectra recorded with a digital resolution of $23.6 \mathrm{~Hz}$ in the indirect dimension so that a clear separation of the carbon resonances of the two steroid parts was visible.

\section{Integration of the NOE data}

For the detection of distance restraints, NOESY spectra were recorded with 10 different mixing times from 60 to $450 \mathrm{~ms}$. We integrated the volume of the cross peaks in the NOESY spectrum at the mixing time of $150 \mathrm{~ms}$. The integrals were calibrated against the NOESY cross-peak integral for the geminal protons at C23' with an assumed internuclear distance of $1.78 \AA .44$ NOE derived distances are listed in Table 6.4. 
Table 6.2: Long-range proton-proton and proton-carbon coupling constants of fibrosterol sulfate A in DMSO- $d_{6}$ at $298 \mathrm{~K}$.

\begin{tabular}{lrll}
\hline Atoms & ${ }^{3} J_{\mathrm{HH}}(\mathrm{Hz})$ & Atoms & ${ }^{3} J_{\mathrm{CH}}(\mathrm{Hz})$ \\
\hline H24'-H23'b & 3.6 & H24-C26 & 3.9 \\
H24'-H23'a & 11.9 & H24-C27 & 4.3 \\
H22'b-H23'a & 11.8 & H24-C24' & 3.2 \\
H22'a-H23'b & 11.9 & & \\
H22'b-H23'b & 4.8 & & \\
H22'a-H23'a & 4.9 & & \\
H22'b-H20' & 10.4 & & \\
H22'a-H20' & 2.6 & & \\
\hline
\end{tabular}

Table 6.3: Residual dipolar couplings of fibrosterol sulfate A in PAN-gel/DMSO.

\begin{tabular}{lrlr}
\hline CH-vector & ${ }^{1} D_{\mathrm{CH}}(\mathrm{Hz})$ & CH-vector & ${ }^{1} D_{\mathrm{CH}}(\mathrm{Hz})$ \\
\hline C1-H1ax & 18.4 & C26'-H26'a & -13.7 \\
C1-H1eq & 3.7 & C26'-H26'b & -12.3 \\
C3-H3ax & 15.3 & C24'-H24' & 19.4 \\
C4-H4ax & 14.3 & C23'-H23'a & 18.7 \\
C4-H4eq & 7.8 & C23'-H23'b & 6.6 \\
C5-H5ax & 20.4 & C22'-H22'a & 5.3 \\
C6-H6ax & 22.3 & C21'-H21' & 0.2 \\
C7-H7ax & 18.7 & C19'-H19' & -6.6 \\
C12-H12ax & 21.0 & C18'-H18' & -5.9 \\
C12-H12eq & 4.2 & C17'-H17' & 21.2 \\
C14-H14ax & 24.2 & C16'-H16'a & 3.9 \\
C16-H16b & 5.8 & C14'-H14'ax & 18.0 \\
C18-H18 & -5.8 & C12'-H12'ax & 22.0 \\
C19-H19 & -6.9 & C11'-H11'ax & 18.5 \\
C20-H20 & 19.7 & C11'-H11'eq & -8.6 \\
C21-H21 & 2.2 & C7'-H7'ax & 22.1 \\
C22-H22 & -3.8 & C6'-H6'ax & 25.7 \\
C23-H23 & 3.6 & C4'-H4'eq & 7.5 \\
C24-H24 & 4.9 & C3'-H3'eq & -25.4 \\
C26-H26 & -1.1 & C2'-H2'eq & 1.5 \\
C27-H27a & 15.3 & C1'-H1'ax & 19.5 \\
C27-H27b & -8.3 & C1'-H1'eq & 11.0 \\
\hline
\end{tabular}


Table 6.4: Proton distance information of fibrosterol sulfate A derived from a NOESY spectrum at $150 \mathrm{~ms}$ mixing time. All distances were referenced to the methylene protons H23'a/H23'b, which were assumed to have a proton distance of $1.78 \AA$.

\begin{tabular}{llll}
\hline Atoms & Distance $(\AA)$ & Atoms & Distance $(\AA)$ \\
\hline H14'-H22 & $3.0-4.0$ & H21-H17 & $2.0-3.0$ \\
H15eq-H22 & $3.0-4.0$ & H21-H22 & $2.5-3.5$ \\
H16'a-H22'a & $2.0-3.0$ & H21-H23 & $2.0-3.0$ \\
H16a-H20 & $2.0-3.0$ & H21-H24 & $3.0-4.0$ \\
H16a-H22 & $2.0-3.0$ & H21-OH22 & $2.5-3.5$ \\
H16a-H24 & $4.0-5.0$ & H21'-H12'eq & $2.0-3.0$ \\
H16a-OH22 & $4.0-5.0$ & H22-H23 & $2.5-3.5$ \\
H16b-H22 & $1.5-2.5$ & H22-H24 & $2.0-3.0$ \\
H16b-H23 & $3.5-4.5$ & H22'a-H16'b & $1.5-2.5$ \\
H16b-H24 & $2.0-3.0$ & H22'b-H24' & $2.0-3.0$ \\
H17-H22 & $2.5-3.5$ & H23-OH22 & $3.0-4.0$ \\
H17-H23 & $2.0-3.0$ & H23'a-H24 & $3.0-4.0$ \\
H17-H24 & $2.5-3.5$ & H23'b-H24 & $2.0-3.0$ \\
H18-H20 & $1.5-2.5$ & H24-OH22 & $3.0-4.0$ \\
H18-H21 & $2.0-3.0$ & H26-H23 & $2.0-3.0$ \\
H18-H22 & $3.0-4.0$ & H26-H24 & $2.5-3.5$ \\
H18'-H21' & $2.0-3.0$ & H21-H26 & $2.5-3.5$ \\
H2-H2' & $4.0-5.5$ & H24'-H24 & $3.0-4.0$ \\
H20-H22 & $1.5-2.5$ & H2-H4'eq & $4.5-5.5$ \\
H20-OH22 & $2.0-3.0$ & H3-H4'eq & $4.0-5.5$ \\
H20'-H22'a & $2.5-3.5$ & H26-H22'a & $2.5-3.5$ \\
H21-H12eq & $1.5-2.5$ & H18-OH22 & $4.5-5.5$ \\
\hline
\end{tabular}




\section{Configurational and conformational analysis}

Configurations for the rigid steroid ring moieties of fibrosterol sulfate A were previously established on the basis of extensive 1D and 2D NMR experiments [Whitson et al., 2009]. In this study we confirmed the configuration of steroid rings ABCD and A'B'C'D' by comparing the similarity of the ${ }^{1} D_{\mathrm{CH}} \mathrm{RDC}$ s of parallel C-H vectors without calculation of the order tensor [Yan et al., 2003] since in the six-membered chair-like ring fragment of both steroid moieties [ABC/A'B'C'] all axial C-H bonds are parallel and therefore have nearly the same angle with respect to the alignment tensor. Thus, the ${ }^{1} D_{\mathrm{CH}}$ couplings coming from axial $\mathrm{C}-\mathrm{H}$ bonds within each of the steroid ring systems should have the same size, which is indeed the case (Table 6.3). However, due to the flexibility of the linker and the relatively small number of coupling constants measured for this region, the configuration of the three stereocenters $\mathrm{C} 22$, C25, and C24' in the linker part remained undetermined. There are eight possible configurations of fibrosterol sulfate A that we refer to using the configuration of C22, C25, and C240: e.g., $S R S$ is the diastereomer that has a configuration of $S$ at $\mathrm{C} 22, R$ at $\mathrm{C} 25$, and $S$ at $\mathrm{C} 24$ '.

Due to the flexibility of the linker, the shape of the entire fibrosterol sulfate A is expected to vary dramatically with linker conformation, and therefore the single alignment tensor approach, which has been extensively applied in previous studies [Schuetz et al., 2007, Sun et al., 2011a, Trigo-Mouriño et al., 2010], is expected to be problematic in this case. Hence, we determined the alignment tensor for each main conformer individually according to Equation 3.6 and Equation 3.7, as described in Chapter 3. Note that only the product of population and alignment tensor can be derived from the experimentally measured RDCs [Burnell and Delange, 1980], and the two unknown parameters cannot be separated unless additional information on either the population of each conformer in the ensemble or the relative sizes of the alignment tensors for each conformer is obtained from independent sources. In a previous study on $\alpha$-methylene- $\gamma$-butyrolactone [Thiele et al., 2009], this problem was solved assuming that the magnitude of the alignment tensors was the same for the two conformers in the ensemble. This is not, however, a valid assumption for fibrosterol sulfate A since the degree of alignment induced by extended or collapsed structures is expected to be very different.

We solved this problem by determining the populations of the representative conformers of clusters from a MD simulation, so that in the fitting procedure only the tensor parameters had to be optimized. To obtain the populations with the best possible accuracy, we performed unrestrained MD simulations for each possible 
configuration of fibrosterol sulfate $\mathrm{A}$ in a DMSO solvent box using the GROMOS 53A6 force field, which is optimized for small molecules. $100 \mathrm{~ns}$ MD simulations at $298 \mathrm{~K}$ produced conformational ensembles that were used to interpret the NMR data. Figure $6.2 \mathrm{a}$ shows the ensemble averaged $\left(\mathrm{r}^{-6}\right.$ averaging) distance violations with respect to the 44 experimental NOEs relevant for the analysis of the configuration of centers 22, 25, and 24' (Table 6.4). Averaging was done over all recorded structures in steps of 5 ns. Few NOEs between the two steroid ring systems could be observed and integrated, due to strong chemical shift degeneracy of the pseudosymmetric bis-steroid. The only difference of the two steroid ring systems arises from the different configuration of $\mathrm{C} 3$ and $\mathrm{C} 3$ ', which results in a significant deviation of the chemical shifts for the H2/H2' and H3/H3' pairs. Nevertheless, three long-range NOEs between the two steroid ring systems H2-H2', H2-H4'eq, and H3-H4'eq could be unambiguously integrated and used in the followed calculation.

Averaged ${ }^{3} \mathrm{~J}$-couplings were calculated over all recorded structures in steps of 5 ns shown in Figure 6.2b. The violations are the averaged deviations from three ${ }^{3} J_{\mathrm{CH}}$ couplings and eight ${ }^{3} J_{\mathrm{HH}}$ couplings (Table 6.2) that are able to define the conformation in the flexible linker region. The time series of the averaged NOE and $J$-coupling violations were calculated for each possible configuration, which are depicted in different colors in Figure 6.2.

For all eight different configurations, we used an energy-minimized starting structure with a fully extended conformation. From Figure 6.2, one can see that the large NOE and ${ }^{3} J$ coupling violations drop rapidly in the first 10 ns for most configurations because during this time collapsed conformations with contacts between the two steroid halves are populated, fulfilling the experimental NOEs and ${ }^{3} J$ couplings better than the extended conformations. Some configurations like $R R S$ and $S R R$ need more time to populate such collapsed conformations. The NOE and ${ }^{3} J$ coupling violations show a similar evolution for most configurations. Comparing the eight possible configurations, the lowest averaged NOE and ${ }^{3} J$ coupling violations were obtained for configuration $S S S$ after 15 ns simulation. To identify the populated conformers of fibrosterol sulfate A in solution, a conformational clustering analysis was performed. Two structures are members in the same cluster if their mutual RMSD of all heavy atom positions is below $0.2 \mathrm{~nm}$. For the conformational clustering, we chose only that part of the trajectory which has the lowest NOE and $J$ coupling violations (Figure 6.2) so that the populations of the main clusters depend not only on the energy derived from the force field but also on the experimental NMR data. As an example, for configuration $S S S$ the ensemble of the structures 
(a)

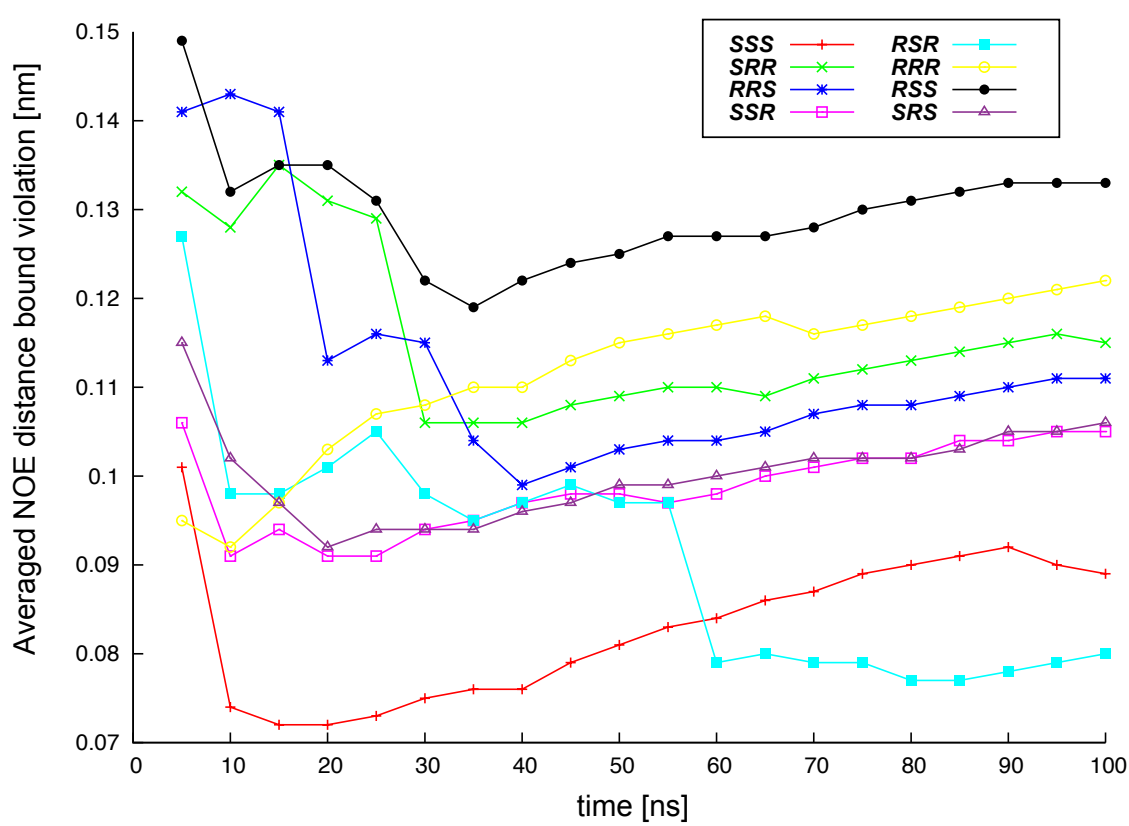

(b)

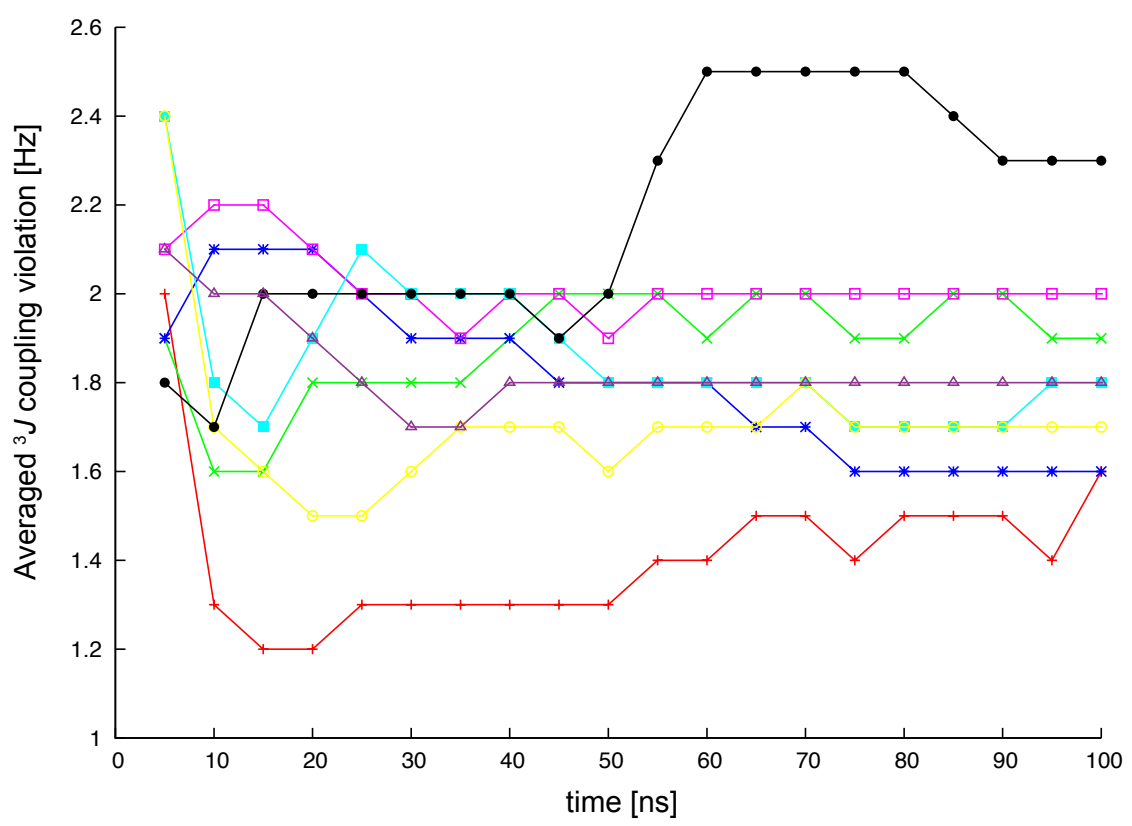

Figure 6.2: (a) Averaged ( $\mathrm{r}^{-6}$ averaging) distance minus the NOE upper or lower-bound distances calculated over all recorded structures after every $5 \mathrm{~ns}$ unrestrained MD simulation. The violations are the averaged deviations from 44 NOEs. (b) Averaged ${ }^{3} J$ coupling violations calculated over all recorded structures after every 5 ns unrestrained MD simulation. The violations shown in the figure are the averaged deviations from three ${ }^{3} J_{\mathrm{CH}}$ couplings and eight ${ }^{3} J_{\mathrm{HH}}$ couplings that are able to define the conformation in the flexible linker region. The unrestrained MD simulations were performed using the GROMOS 53A6 force field at $298 \mathrm{~K}$. 
Table 6.5: Multiple alignment tensor fit: the populations of the main conformations and $Q$ factor of each configuration using the RDC fitting to a structure ensemble. The alignment tensors were determined individually from $44{ }^{1} D_{\mathrm{CH}}$ for each conformer using the SVD method. The populations were calculated using a conformational clustering analysis on a selected unrestrained MD trajectory based on NOEs and ${ }^{3} J$ couplings.

\begin{tabular}{llllll}
\hline Configuration & \multicolumn{4}{c}{ Population } & $Q$ factor \\
& Conf. 1 & Conf. 2 & Conf. 3 & Conf. 4 \\
\hline$R S R$ & 0.57 & 0.25 & 0.13 & 0.05 & 0.25 \\
$R R S$ & 0.51 & 0.24 & 0.15 & 0.10 & 0.33 \\
$S S R$ & 0.39 & 0.33 & 0.17 & 0.11 & 0.36 \\
$S R S$ & 0.68 & 0.26 & 0.05 & 0.01 & 0.37 \\
$R R R$ & 0.65 & 0.19 & 0.10 & 0.06 & 0.26 \\
$R S S$ & 0.60 & 0.18 & 0.12 & 0.10 & 0.34 \\
$S R R$ & 0.40 & 0.29 & 0.19 & 0.12 & 0.43 \\
$S S S$ & 0.33 & 0.48 & 0.09 & 0.10 & 0.13 \\
\hline
\end{tabular}

from the first $15 \mathrm{~ns}$ of the full trajectory, which has the lowest averaged NOE and ${ }^{3} J$ coupling violations, was selected for the clustering. In the clustering analysis, we found that for each possible configuration only four clusters are significantly populated (Table 6.5).

Representative structures of the four most populated clusters for each configuration were manually selected and weighted with the determined populations, and their individual alignment tensors were determined by fitting against the 44 experimental ${ }^{1} D_{\mathrm{CH}}$ couplings. This amounted to the determination of 4 (conformations) times 5 (components of the alignment tensor) $=20$ unknowns fitted to the 44 knowns. The fit was accomplished by solving the linear equation system using the SVD method (Equation 3.7). 20 non-zero tensor elements were obtained and the condition number of this linear equation function is 51. The correct configuration $S S S$ was selected based on the lowest $Q$ factor (0.13) for experimental and back-calculated RDCs, which is summarized in Table 6.5 and Figure A.17. The other 7 configurations yielded significantly larger $Q$ factors. The four representative cluster conformations of the configuration SSS (Figure 6.3) contain a collapsed conformation (conformer 1 ), an extended conformation (conformer 2), and two L-shaped conformations (conformers 3 and 4). The dihedrals of each rotatable bond in the linker reported for each conformation (Figure 6.3) are listed in Table A.19.

Although in our multiple-tensor analysis we fitted 20 independent parameters, 


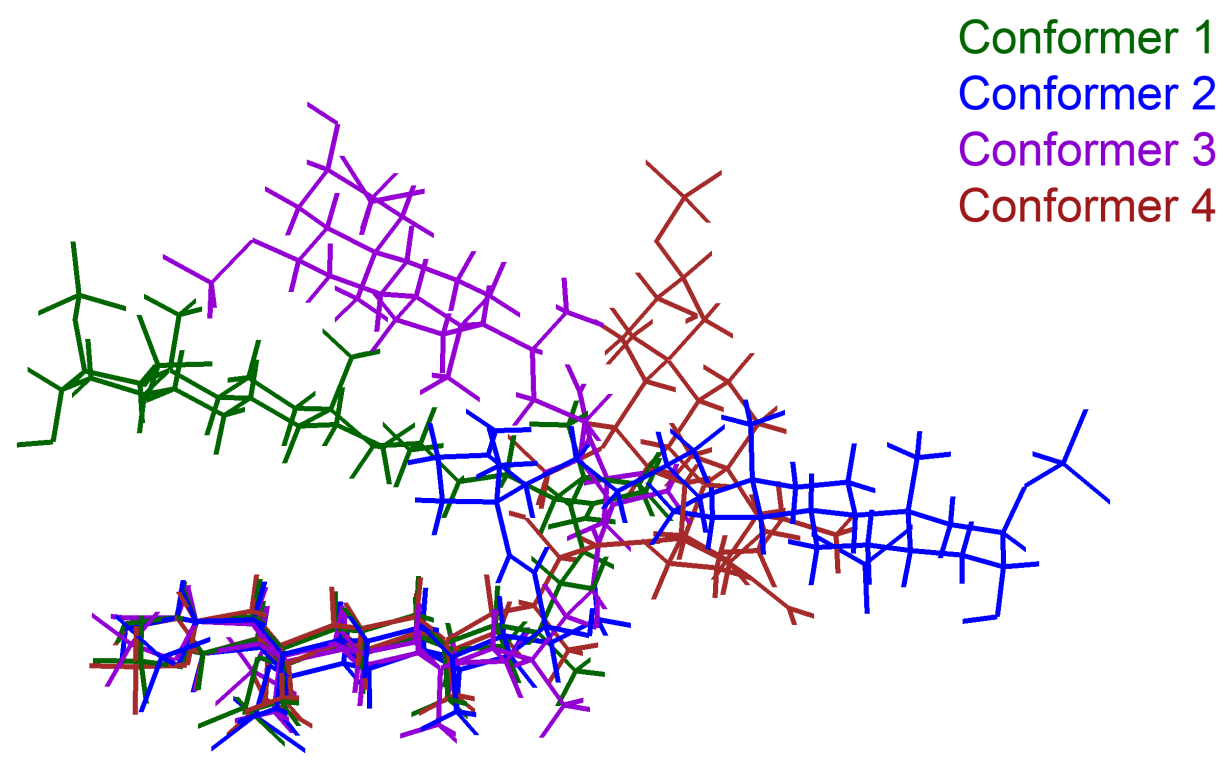

Figure 6.3: Multiple alignment tensor fit: the four populated conformations of the bestfitting configuration $S S S$, superimposed on steroid ABCD. The SVD fitting of the $44{ }^{1} D_{\mathrm{CH}}$ was performed against four conformers for $S S S$ configuration. The four main conformers and the populations were obtained from a selected unrestrained MD simulation based on NOE and ${ }^{3} J$ couplings. The alignment tensor was determined individually for each conformer.

coming from four order tensors, to 44 experimental ${ }^{1} D_{\mathrm{CH}}$ values, some of them can be redundant due to nearly parallel $\mathrm{CH}$ vectors in the rigid steroid body. Therefore, the fitting process could be effectively underdetermined, making it necessary to perform an error analysis. Here, we performed a bootstrapping Monte-Carlo analysis, in which 250 sets of randomly generated RDC data were used as the input data for the fitting on the given structures. In each data set, a Gaussian distributed noise with a standard deviation of $0.5 \mathrm{~Hz}$ was assumed. This standard deviation corresponds to a maximum RDC error of about $1.5 \mathrm{~Hz}$ for the $99 \%$ limits of confidence, which was estimated according to the signal-to-noise ratio of the isotropic and anisotropic $\left[{ }^{1} \mathrm{H},{ }^{13} \mathrm{C}\right]$-CLIP-HSQC spectra. The error analysis was performed for each possible configuration, and each point in Figure 6.4 reveals the $Q$ factor of one fitting procedure for one configuration. Since the distribution of $Q$ values for configuration $S S S$ over the expected experimental uncertainty ranges does not overlap with the $Q$ distribution of other configurations [García et al., 2009], the selection of the $S S S$ configuration over the others is significant within the experimental error.

The orientations of the principle axes of the calculated alignment tensor for each conformer of the best-fitting configuration $S S S$ are depicted in Figure 6.5, and the 


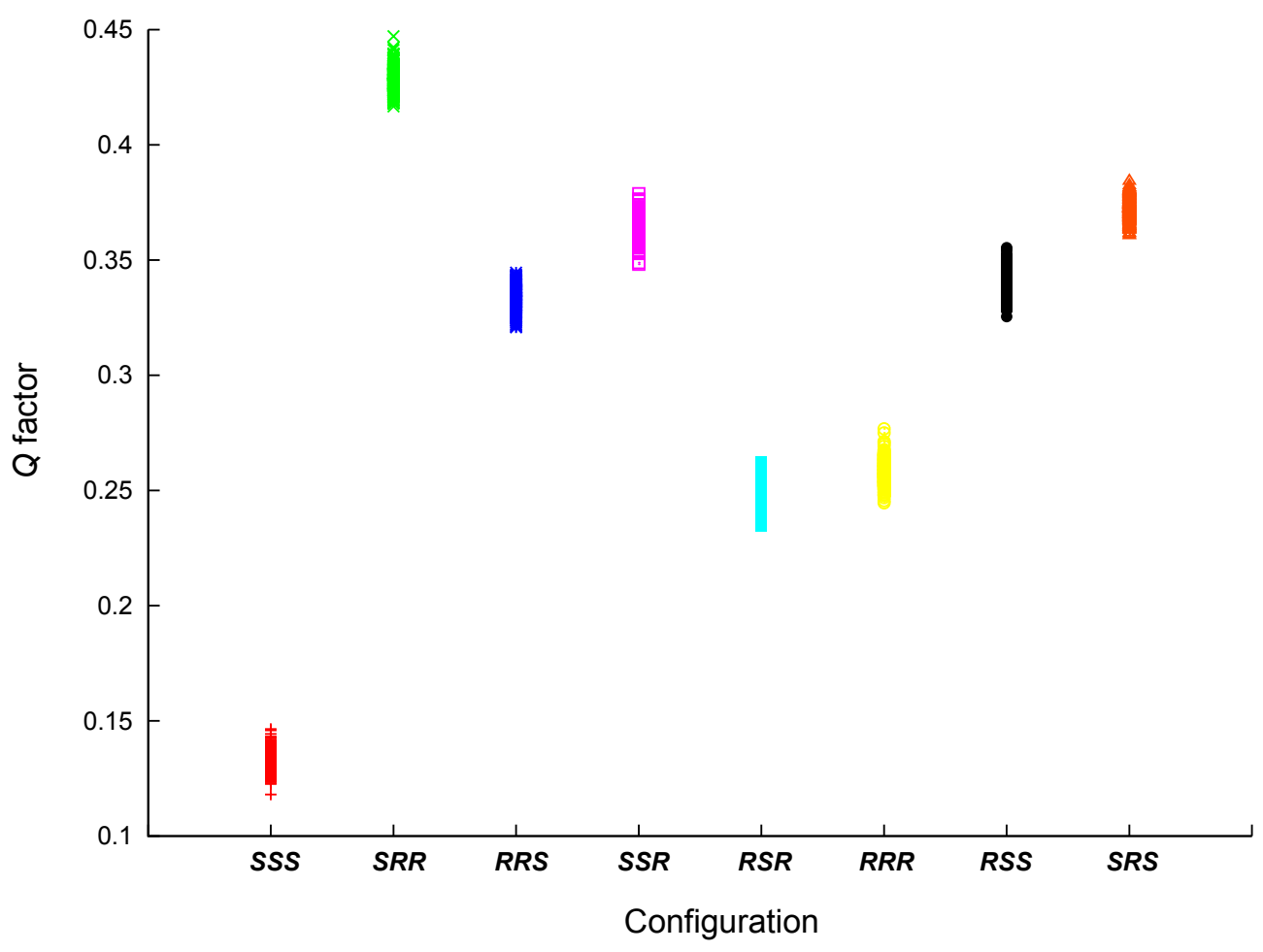

Figure 6.4: Multiple alignment tensor fit: the error bars of the $Q$ factors of eight possible configurations obtained by bootstrapping error analysis using a standard deviation value of $0.5 \mathrm{~Hz}$.

order parameters are shown in Table 6.6. These parameters give the information about the preferential orientation and the magnitude of the alignment of different conformers. The chosen alignment medium was a stretched poly(acrylonitrile) gel, which contains a nitrile group in the main chain of the polymer. Since fibrosterol sulfate A contains four charged sodium sulfate groups, both steric and electrostatic interactions between the solute and the gel can contribute to the alignment mechanism. Therefore, prediction of the alignment tensor for each conformation only from the 3D structure of the molecule [Zweckstetter and Bax, 2000, Almond and Axelsen, 2002, Azurmendi and Bush, 2002] becomes particularly difficult and was therefore not performed for this study.

To evaluate the errors on each derived tensor parameter and their correlations, we calculated the scaled and averaged variance-covariance matrix of fitted parameters for the best fitting configuration $S S S$, which are shown in Figure A.18 - A.21. The scaled variance-covariance matrix [Lawson and Hanson, 1974] is given by:

$$
\mathbf{C}=\frac{\mathbf{V S}^{-2} \mathbf{V}^{\mathbf{T}}}{\kappa^{2}} \cdot \frac{\|\mathbf{M A}-\mathbf{D}\|^{2}}{m-n}
$$


Table 6.6: Multiple alignment tensor fit: alignment tensor parameters of each conformer of the best-fitting configuration $S S S . \mathrm{A}_{x x}, \mathrm{~A}_{y y}$ and $\mathrm{A}_{z z}$ are the eigenvalues of the alignment tensor; $\mathrm{A}_{a}$ and $\mathrm{A}_{r}$ are axial and rhombic component of the alignment tensor, respectively; $\alpha, \beta$ and $\gamma$ are Euler angles in degrees for rotation of the alignment tensor into the principle axis frame. The reference frame is the $x y z$ coordinate frame. The alignment tensor was determined individually from $44{ }^{1} D_{\mathrm{CH}}$ couplings for each conformer using the SVD method.

\begin{tabular}{lcccc}
\hline Parameter & \multicolumn{4}{c}{ Conformer } \\
& 1 & 2 & 3 & 4 \\
\hline $\mathrm{A}_{x x}$ & $-7.33 \times 10^{-5}$ & $4.58 \times 10^{-5}$ & $-4.44 \times 10^{-4}$ & $1.69 \times 10^{-4}$ \\
$\mathrm{~A}_{y y}$ & $-2.88 \times 10^{-4}$ & $1.15 \times 10^{-3}$ & $-1.31 \times 10^{-3}$ & $1.62 \times 10^{-3}$ \\
$\mathrm{~A}_{z z}$ & $3.61 \times 10^{-4}$ & $-1.19 \times 10^{-3}$ & $-1.76 \times 10^{-3}$ & $-1.79 \times 10^{-3}$ \\
$\mathrm{~A}_{a}$ & $5.42 \times 10^{-4}$ & $-1.79 \times 10^{-3}$ & $2.64 \times 10^{-3}$ & $-2.68 \times 10^{-3}$ \\
$\mathrm{~A}_{r}$ & $2.15 \times 10^{-4}$ & $-1.10 \times 10^{-3}$ & $8.70 \times 10^{-4}$ & $-1.45 \times 10^{-3}$ \\
$\alpha$ & 24.8 & -66.9 & -81.9 & -10.2 \\
$\beta$ & 47.5 & 5.1 & -6.2 & 11.0 \\
$\gamma$ & -38.0 & -65.0 & -49.9 & 25.9 \\
\hline
\end{tabular}

where $\mathbf{M}$ is a matrix composed of direction cosines of the internuclear vectors, $\mathbf{A}$ containes the tensor parameters for each conformer, $\mathbf{D}$ is a vector composed of the experimental RDCs, $\kappa$ is defined as: $\kappa=-3 /\left(16 \pi^{3}\right) \gamma_{I} \gamma_{S} \mu_{0} h, \mathbf{V}$ is the $\mathrm{V}$-matrix and $\mathbf{S}$ the singular values of $\mathbf{M}$ obtained by decomposition of $\mathbf{M}$ with SVD, and $\mathrm{m}$ is the rank of $\mathbf{D}$ and $\mathrm{n}$ is the rank of $\mathbf{A}$. The averaged variance-covariance matrix is given by:

$$
\mathbf{C}=\frac{\mathbf{V S}^{-2} \mathbf{V}^{\mathbf{T}}}{\kappa^{2} m} \cdot \frac{\|\mathbf{M A}-\mathbf{D}\|^{2}}{m-n} .
$$

The standard deviations of the tensor parameters, given in Table 6.7, were calculated by taking the square root of the variance. The standard deviations of the mean of the tensor parameters for each conformer are one order of magnitude smaller than the tensor parameter with the largest magnitude, indicating the magnitude and the main axis of the tensor can be determined accurately. The fact that the small (conformer 1 and 2) and the large tensors (conformer 3 and 4) have the same relative standard deviation indicates that the errors in the small tensors are not dominated by the cross-variance from the large tensors.

To conclude this part of our work, we have presented a new approach using $\mathrm{RDC}$ data to establish the relative configuration and study the conformation of the 
Table 6.7: The standard deviation of the tensor parameters, derived from the variancecovariance matrix of the best-fitting configuration SSS.

\begin{tabular}{llll}
\hline & & Tensor parameter & Standard deviation \\
\hline Conformer 1 & $\mathrm{A}_{x y}$ & $-7.7 \times 10^{-5}$ & $9.5 \times 10^{-6}$ \\
& $\mathrm{~A}_{x z}$ & $-1.8 \times 10^{-5}$ & $7.4 \times 10^{-6}$ \\
& $\mathrm{~A}_{y y}$ & $-3.3 \times 10^{-5}$ & $1.0 \times 10^{-5}$ \\
& $\mathrm{~A}_{y z}$ & $-1.9 \times 10^{-4}$ & $1.1 \times 10^{-5}$ \\
& $\mathrm{~A}_{z z}$ & $-1.2 \times 10^{-4}$ & $8.5 \times 10^{-6}$ \\
\hline Conformer 2 & $\mathrm{A}_{x y}$ & $-6.6 \times 10^{-4}$ & $1.8 \times 10^{-5}$ \\
& $\mathrm{~A}_{x z}$ & $5.4 \times 10^{-6}$ & $2.5 \times 10^{-5}$ \\
& $\mathrm{~A}_{y y}$ & $2.4 \times 10^{-4}$ & $2.5 \times 10^{-5}$ \\
& $\mathrm{~A}_{y z}$ & $2.4 \times 10^{-4}$ & $3.6 \times 10^{-5}$ \\
& $\mathrm{~A}_{z z}$ & $4.0 \times 10^{-4}$ & $4.4 \times 10^{-5}$ \\
\hline Conformer 3 & $\mathrm{A}_{x y}$ & $9.0 \times 10^{-4}$ & $5.7 \times 10^{-5}$ \\
& $\mathrm{~A}_{x z}$ & $-1.1 \times 10^{-3}$ & $5.2 \times 10^{-5}$ \\
& $\mathrm{~A}_{y y}$ & $-3.9 \times 10^{-4}$ & $4.4 \times 10^{-5}$ \\
& $\mathrm{~A}_{y z}$ & $3.7 \times 10^{-4}$ & $7.3 \times 10^{-5}$ \\
& $\mathrm{~A}_{z z}$ & $5.1 \times 10^{-4}$ & $5.0 \times 10^{-5}$ \\
\hline Conformer 4 & $\mathrm{A}_{x y}$ & $1.3 \times 10^{-3}$ & $5.5 \times 10^{-5}$ \\
& $\mathrm{~A}_{x z}$ & $-5.7 \times 10^{-4}$ & $8.6 \times 10^{-5}$ \\
& $\mathrm{~A}_{y y}$ & $2.9 \times 10^{-4}$ & $5.6 \times 10^{-5}$ \\
& $\mathrm{~A}_{y z}$ & $-3.2 \times 10^{-4}$ & $5.8 \times 10^{-5}$ \\
& $\mathrm{~A}_{z z}$ & $-3.1 \times 10^{-4}$ & $3.9 \times 10^{-5}$ \\
\hline & & & \\
\hline
\end{tabular}



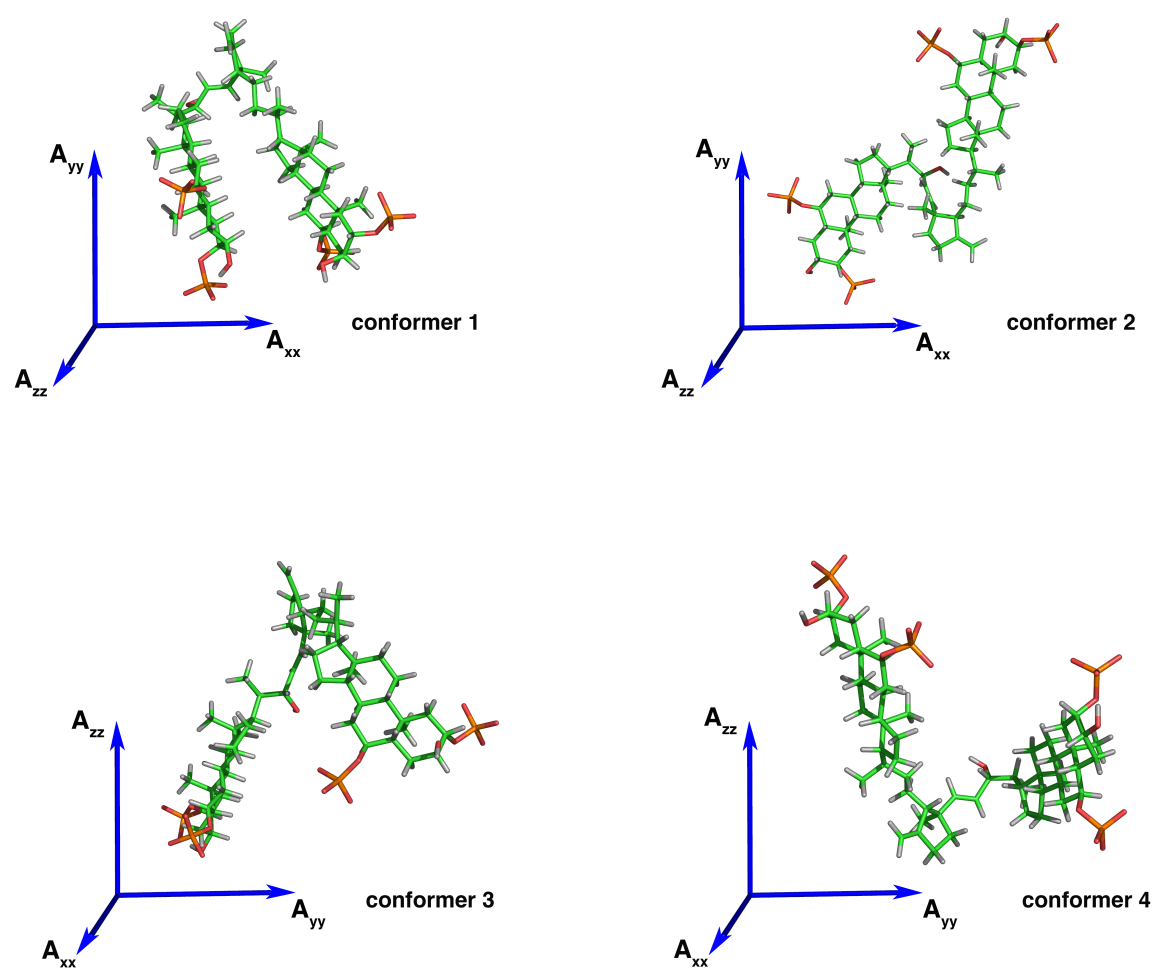

Figure 6.5: Multiple alignment tensor fit: orientations of four conformers of $S S S$ configuration in the principal axis frame of the individual alignment tensor. The three blue arrows indicate the principal axes with components $\mathrm{A}_{x x}, \mathrm{~A}_{y y}$, and $\mathrm{A}_{z z}$ of the molecular order tensors, respectively.

molecule. We first determined the population from a MD trajectory evaluated for consistency with the experimental NOEs and ${ }^{3} J$ values. A subsequent RDC-based analysis was then undertaken in which the alignment tensor was calculated individually for each populated conformer. The schematic representation of the entire analysis procedure is shown Figure 6.6.

\subsubsection{Elucidation of the relative configuration and conforma- tion by single-tensor analysis}

To assess whether the more complex multi-tensors approach provides any advantages in the case of fibrosterol sulfate A we have also performed the analysis assuming a single alignment tensor for all possible configurations. Unlike in the multiple tensors approach, the populations of the conformer and tensor parameters are linearly independent. The populations can therefore be determined together with the five order parameters of the tensor applied to all conformers. 


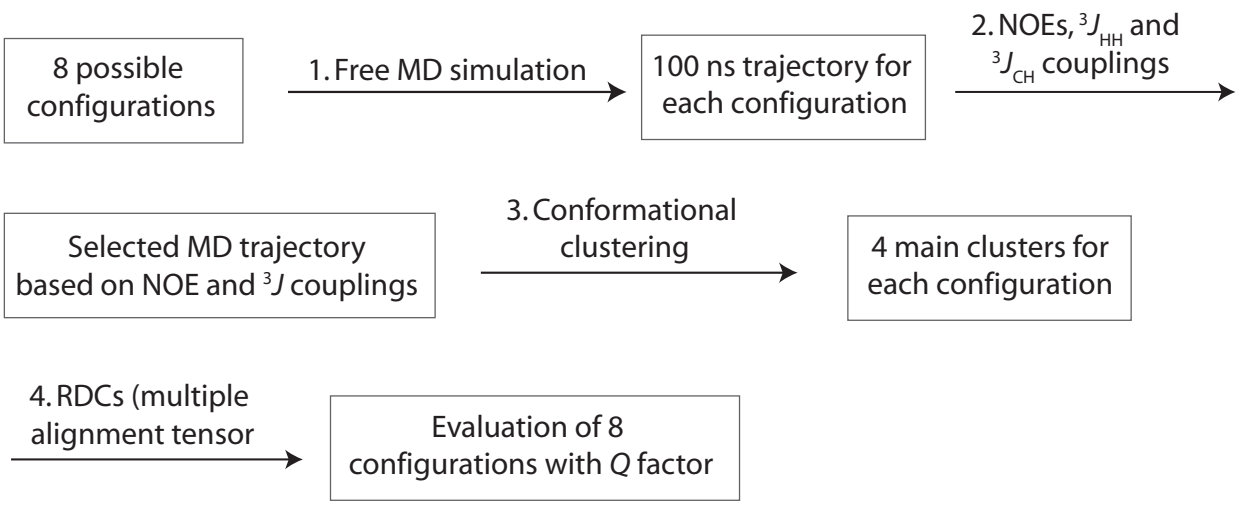

Figure 6.6: Schematic representation of the RDC-based multiple-tensor analysis used for configurational and conformational studies of fibrosterol sulfate A. 1. Free MD is unrestrained MD. 2. NOEs, ${ }^{3} J_{\mathrm{HH}}$ couplings and ${ }^{3} J_{\mathrm{CH}}$ couplings means that the part of the trajectory is selected that shows the least violations of experimental parameters. The selected trajectory for $S S S$ ran from 0 to $15 \mathrm{~ns}$.

To sample the conformational space of the molecule efficiently, we performed a simulated annealing simulation without any NMR restraints to generate 200 structures for each configuration. As an example, Figure 6.7 shows 200 structures of configuration $S S S$ superimposed on the steroid ABCD, which is visualized in blue. From Figure 6.7 it is obvious that the sampled conformations from the simulated annealing are highly divergent, suggesting that a thorough sampling of the conformational space, as required, has been achieved.

${ }^{2,3} \mathrm{~J}$-couplings allow unambiguous determination of local conformations if they adopt extreme values. This is the case for the C22'-C23' dihedral angle, in which we obtain two large ${ }^{3} J_{\mathrm{HH}}$ couplings $\left({ }^{3} J_{\mathrm{H} 22^{\prime} \mathrm{b}-\mathrm{H} 23^{\prime} \mathrm{a}}\right.$ and ${ }^{3} J_{\mathrm{H} 22^{\prime} \mathrm{a}-\mathrm{H} 23^{\prime} \mathrm{b}}$ around $\left.12 \mathrm{~Hz}\right)$ and two small couplings $\left({ }^{3} J_{\mathrm{H} 22^{\prime} \mathrm{b}-\mathrm{H} 23^{\prime} \mathrm{b}}\right.$ and ${ }^{3} J_{\mathrm{H} 22^{\prime} \mathrm{a}-\mathrm{H} 23^{\prime} \mathrm{a}}$ around $5 \mathrm{~Hz}$ ) (Table 6.2). According to the Karplus equation (Equation 2.45) this pattern clearly indicates that C20' and C24' are antiperiplanar with respect to each other. This is the same side chain conformation found in a previous study of a structurally related steroid [Sun et al., 2010]. Due to the extreme ${ }^{3} J_{\mathrm{H} 22^{\prime}-\mathrm{H} 23^{\prime}}$ values, we can exclude from further analysis all conformations that don't have an anti conformation for the C20'-C22'C23'-C24' moiety. More than $75 \%$ of the 200 structures from the simulated annealing simulations could be excluded for all configurations (Table A.20 and Figure A.22).

Note however, that the remaining conformations may still contain other incorrect conformations due to insufficient ${ }^{2,3} J$-coupling restraints. In order to simplify 


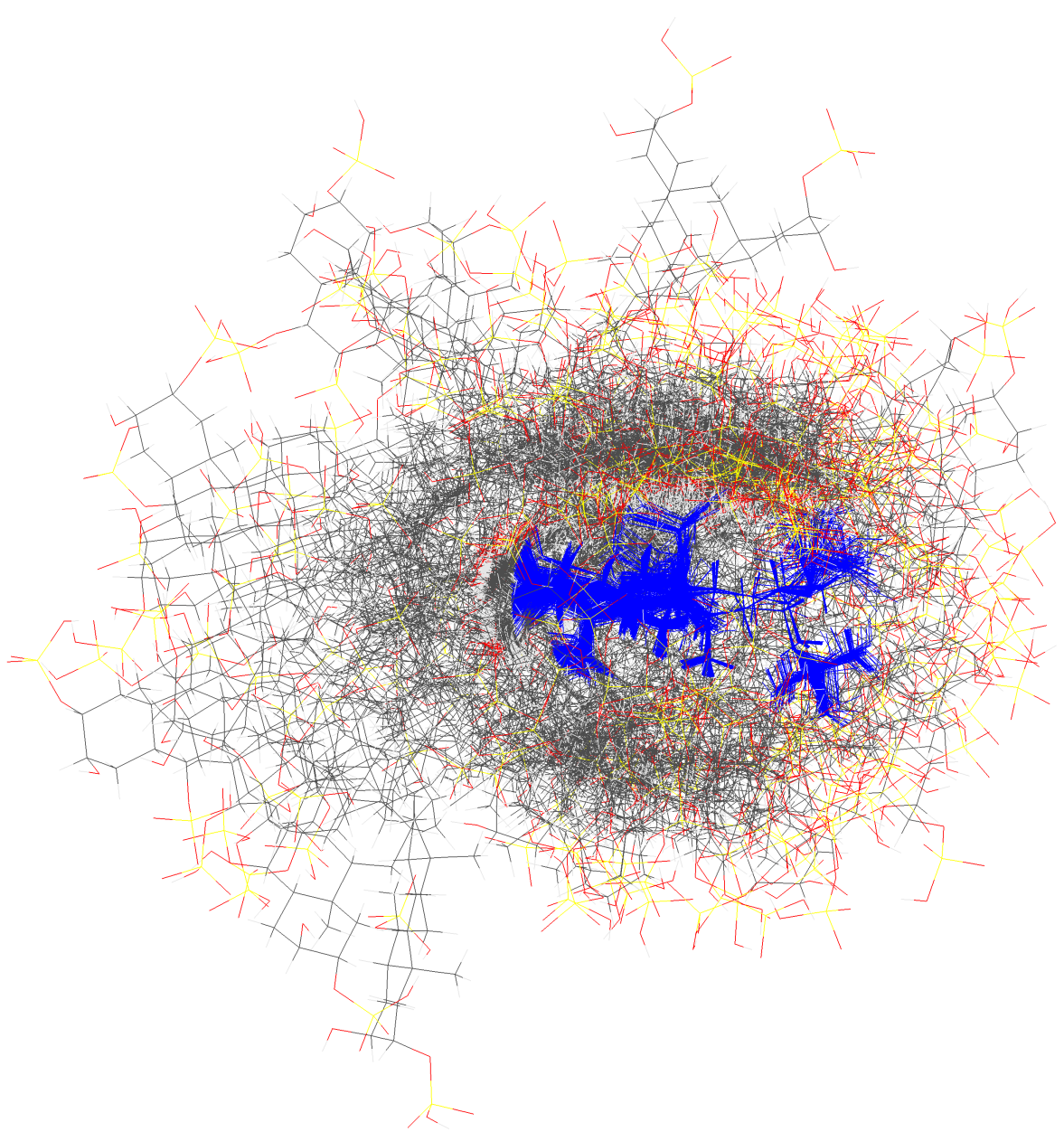

Figure 6.7: 200 structures of $S S S$ sampled by a simulated annealing simulation, are superimposed on the ABCD steroid ring system, and shown in blue.

the system further we selected the ${ }^{1} D_{\mathrm{CH}}$ couplings from the steroid ABCD and the linker part and fitted the data set to each remaining structure individually using the SVD method. Together the two regions account for 30 RDCs, and thus should be sufficient for determining the five parameters defining the alignment tensor. Finally, we ranked the conformations according to the $Q$ factor between experimental and theoretically calculated RDCs. The five best structures of each configuration were selected and their $Q$ factors are shown in Figure 6.8. Among the 8 possible configurations, $S S S$ shows the lowest average $Q$ factor for the five best structures. It is also important to note that the best structure of configuration $S S S$ has a superior fit $(Q=0.109)$ to the RDC data compared to the other configurations.

The five structures of each configuration in Figure 6.8 are consistent with the ${ }^{3} J_{\mathrm{HH}}$ couplings and at the same time provide the best fit to the selected RDCs. Next 


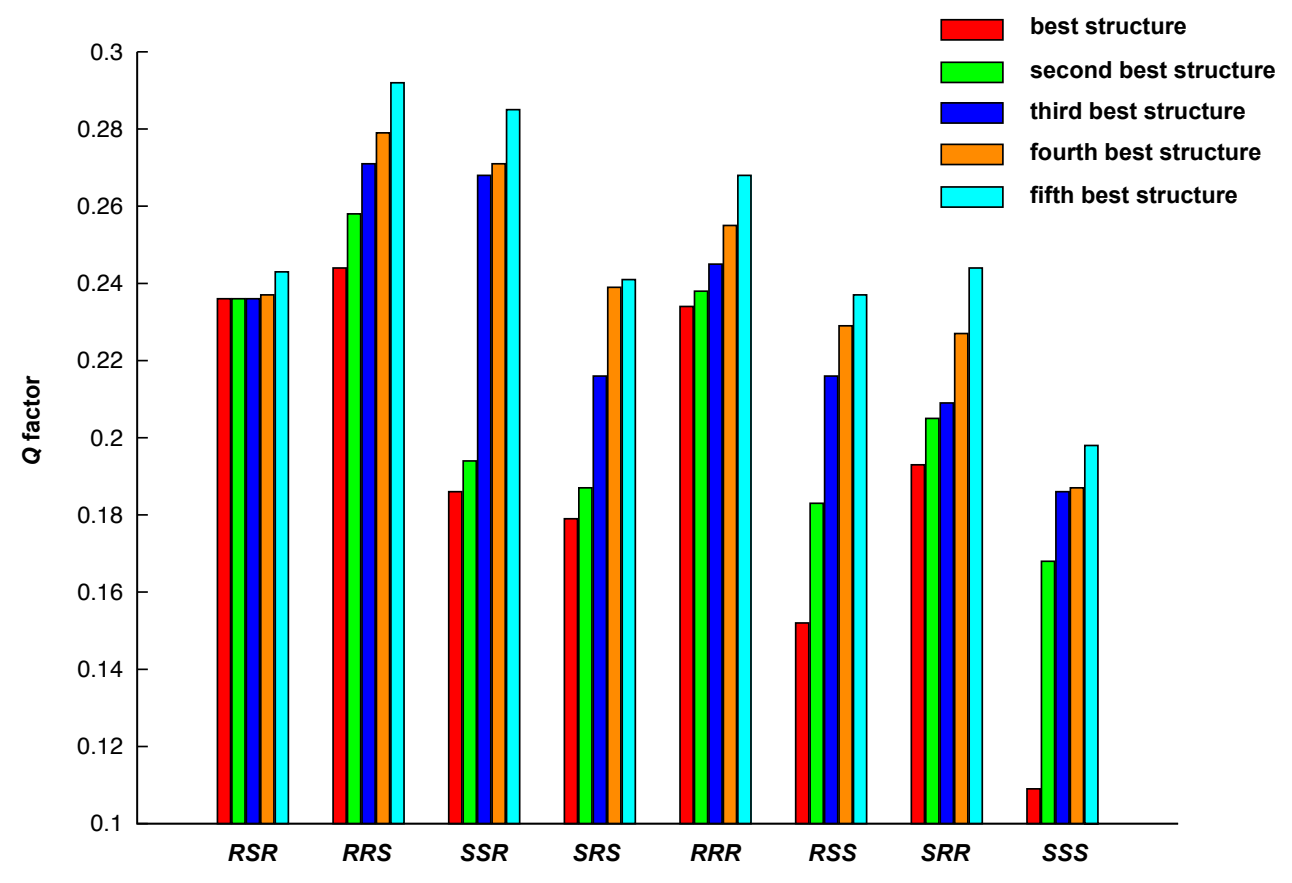

Figure 6.8: Single alignment tensor fit: Comparison of the $Q$ factors of the RDC fitting of the five best structures between 8 configurations. The RDC fitting was performed on a subgroup of experimental RDCs, which includes the data from one steroid ABCD and the linker region.

we undertook to fit each set of conformers as an ensemble to the complete set of experimental RDCs. Here we used the assumption that all the conformations in an ensemble have the same alignment tensor, requiring the superimposition of each conformer to the mean structure [Schuetz et al., 2007, Sun et al., 2011a, Trigo-Mouriño et al., 2010, Thiele et al., 2009] or to a chosen rigid moiety [Sánchez-Pedregal et al., 2009]. For this study we superimposed the five best structures of each configuration to one steroid ring system ABCD. After superimposition the fitting of the ensemble of the structures was performed against the complete set of experimental ${ }^{1} D_{\mathrm{CH}}$ couplings. The populations of the five selected structures, which led to four other independent parameters, were optimized simultaneously with the alignment tensor, thus giving nine parameters to be determined overall. The best fit was again obtained for configuration $S S S$, which has a $Q$ factor of 0.16 (Table 6.8 and Figure A.23). In the final ensemble of conformers for configurations $R S R, R R S$, and $S S R$, only two conformations are populated, whereas in the other configurations there are three (Table 6.8).

From Figure 6.9 one can see that the best-fitting configuration SSS contains one extended (conformer 2) and two collapsed conformations (conformer 1 and 3). 
Table 6.8: Single alignment tensor fit: The populations of the conformations and $Q$ factor of each configuration using the RDC fitting to a structure ensemble assuming a single alignment tensor for different conformations within one ensemble. The relative orientation of conformers in the ensemble is determined by superimposing each structure to one steroid ring system $\mathrm{ABCD}$.

\begin{tabular}{|c|c|c|c|c|}
\hline \multirow[t]{2}{*}{ Configuration } & \multicolumn{3}{|c|}{ Population } & \multirow{2}{*}{$Q$ factor } \\
\hline & Conf. 1 & Conf. 2 & Conf. 3 & \\
\hline$R S R$ & 0.16 & 0.84 & - & 0.33 \\
\hline$R R S$ & 0.60 & 0.40 & - & 0.33 \\
\hline$S S R$ & 0.45 & 0.55 & - & 0.45 \\
\hline$S R S$ & 0.45 & 0.25 & 0.30 & 0.46 \\
\hline$R R R$ & 0.41 & 0.21 & 0.37 & 0.28 \\
\hline$R S S$ & 0.27 & 0.30 & 0.43 & 0.33 \\
\hline$S R R$ & 0.59 & 0.13 & 0.28 & 0.21 \\
\hline$S S S$ & 0.26 & 0.45 & 0.29 & 0.16 \\
\hline
\end{tabular}

The two collapsed conformers nevertheless differ from each other, particularly in the linker region. The low $Q$ factor value of the determined ensemble of configuration $S S S$ indicates that the presence of these three conformations is needed to satisfy the complete experimental RDC data simultaneously. Furthermore, reintroduction of the filtered conformers in the $S S S$ ensemble does not lead to further lowering of the $Q$ factor. This result implies that the three structures shown in Figure 6.9 are both necessary and sufficient for explaining the RDC data using the single tensor analysis. For a comparison with the multiple-tensors analysis, the orientation of the principle axis of the calculated alignment tensor for each conformer of the best-fitting configuration $S S S$ is depicted in Figure 6.10, and the order parameters are shown in Table 6.9.

Furthermore, we have calculated the ensemble NOE violation for each configuration. From Table 6.10 one can infer that the $S S S$ ensemble has the lowest NOE violation, which is consistent with the RDC analysis. The second best RDC fitting and second lowest NOE violation was obtained simultaneously for the configuration $S R R$. The finding that these two configurations have a relatively small difference in the violation is not very surprising, because the only difference of these two configurations is the relative configuration of the stereocenters C22 and the ones in the five-membered ring. Since these two stereochemical domains are relatively far apart from each other, the correlation between them is small and thus difficult to be distinguished on the basis of the ${ }^{1} D_{\mathrm{CH}}$ couplings and NOEs. The other six possible 


\section{Conformer 1}

Conformer 2

Conformer 3

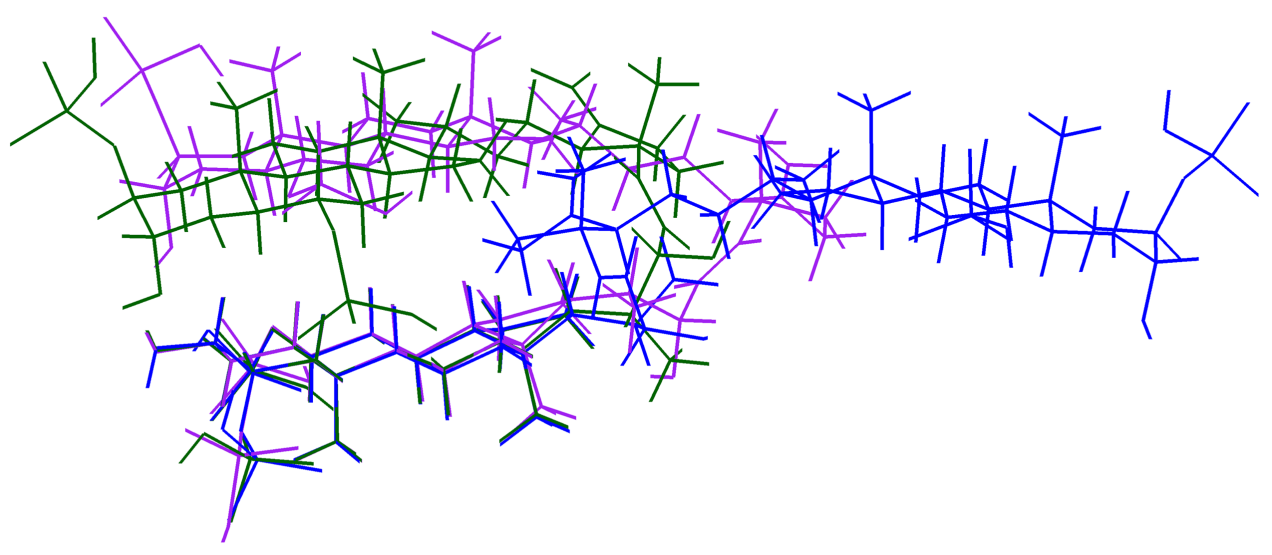

Figure 6.9: Single alignment tensor fit: Three populated conformations of the best-fitting configuration $S S S$, superimposed on the steroid ABCD. The SVD fitting of the $44{ }^{1} D_{\mathrm{CH}}$ couplings was performed against an ensemble of five structures, which are superimposed to the steroid ABCD. One alignment tensor for all structures within one ensemble is assumed.

configurations have far worse agreement with both RDC and NOE data. Therefore the number of possible configurations can now be narrowed down from eight to two. In order to confirm the $S S S$ configuration as the most probable configuration, we performed a cross-validation of the ensemble of structures from RDC analysis for both configurations $S S S$ and $S R R$ against the ${ }^{3} J_{\mathrm{CH}}$ data that were not used so far. The experimental ${ }^{3} J_{\mathrm{CH}}$ data $\left({ }^{3} J_{\mathrm{C} 26-\mathrm{H} 24}=3.9 \mathrm{~Hz},{ }^{3} J_{\mathrm{C} 27-\mathrm{H} 24}=4.3 \mathrm{~Hz}\right.$, ${ }^{3} J_{\mathrm{C} 24^{\prime}-\mathrm{H} 24}=3.2 \mathrm{~Hz}$, listed in Table 6.2) show clearly that conformational averaging exists for the C24-C25 dihedral angle. This finding is in good agreement with the three populated conformations of the $S S S$ configuration, but not with the three $S R R$ conformations, as shown in Table 6.11. As a result, the full analysis described above demonstrates that only the $S S S$ configuration fulfills the RDC data in a single alignment tensor fitting, the ${ }^{3} J$ couplings and the NOEs simultaneously.

The same analysis has been repeated by starting with steroid A'B'C'D' in the first step instead of steroid ABCD. The same configuration $S S S$ is found to be optimal. And again three conformations that have the extended and collapsed shape were found to be mainly populated (Figure A.25). However, a larger $Q$ factor for $S S S(Q=0.22$, Table A.21) was obtained in comparison with $Q=0.16$ based on steroid ABCD (Table 6.8). This seems to be a local effect of the dipolar couplings of steroid A'B'C'D' rather than a weakness of the single alignment tensor approach: 
Table 6.9: Single alignment tensor fit: Alignment tensor parameters of the best-fitting configuration $S S S . A_{x x}, A_{y y}$ and $A_{z z}$ are the eigenvalues of the alignment tensor; $\mathrm{A}_{a}$ and $\mathrm{A}_{r}$ are axial and rhombic component of the alignment tensor, respectively; $\alpha, \beta$ and $\gamma$ are Euler angles in degrees for rotation of the alignment tensor into the principle axis frame. The reference frame is the $x y z$ coordinate frame. A uniform alignment tensor for different conformations within one ensemble was calculated using $44{ }^{1} D_{\mathrm{CH}}$ couplings.

\begin{tabular}{lr}
\hline Parameter & \\
\hline $\mathrm{A}_{x x}$ & $-1.54 \times 10^{-4}$ \\
$\mathrm{~A}_{y y}$ & $-3.51 \times 10^{-4}$ \\
$\mathrm{~A}_{z z}$ & $5.05 \times 10^{-4}$ \\
$\mathrm{~A}_{a}$ & $7.58 \times 10^{-4}$ \\
$\mathrm{~A}_{r}$ & $1.99 \times 10^{-4}$ \\
$\alpha$ & 30.65 \\
$\beta$ & 37.86 \\
$\gamma$ & -3.83 \\
\hline
\end{tabular}

Table 6.10: Single alignment tensor fit: The NOE violations of the 8 possible configurations. A total number of 44 NOEs were used in the calculation.

\begin{tabular}{ll}
\hline Configuration & NOE violations $\left(\AA^{2}\right)$ \\
\hline$R S R$ & 48.6 \\
$R R S$ & 37.2 \\
$S S R$ & 39.3 \\
$S R S$ & 92.8 \\
$R R R$ & 73.5 \\
$R S S$ & 28.7 \\
$S R R$ & 24.7 \\
$S S S$ & 23.9 \\
\hline
\end{tabular}



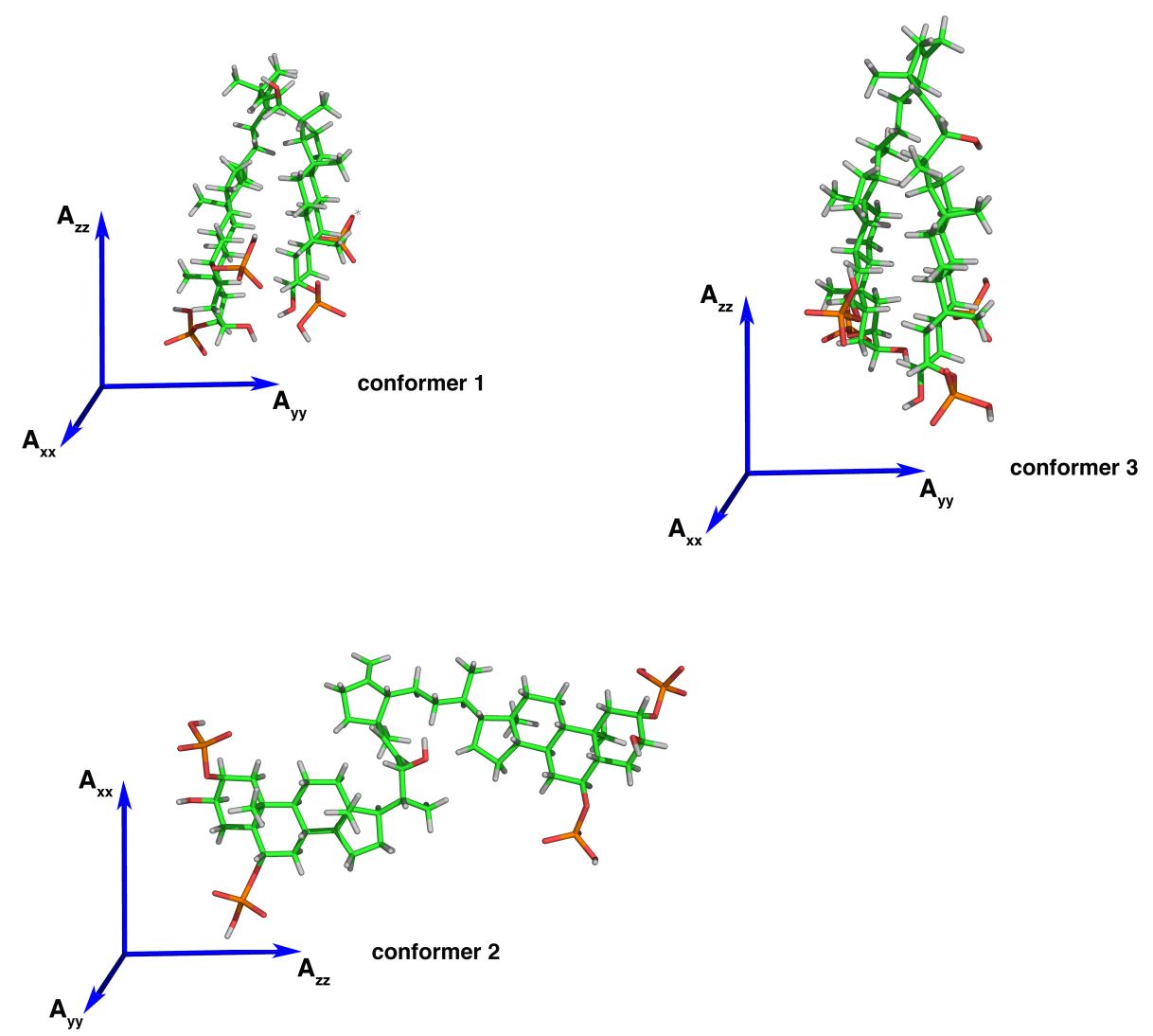

Figure 6.10: Single alignment tensor fit: Orientations of 3 conformers of $S S S$ configuration in the principle axis frame. The three blue arrows indicate the principle axes with components $\mathrm{A}_{x x}, \mathrm{~A}_{y y}$ and $\mathrm{A}_{z z}$ of the molecular order tensors, respectively. A uniform alignment tensor for different conformations within one ensemble was calculated using 44 ${ }^{1} D_{\mathrm{CH}}$ couplings.

The $Q$ factors (0.17-0.24, Figure A.24) of the five best conformations of $S S S$ configuration derived from the RDCs from steroid A'B'C' $D$ ' and the linker region are significantly larger than the respective $Q$ factors for steroid $\operatorname{ABCD}(Q=0.11-0.20$, Figure 6.8).

To summarize the single-tensor analysis of fibrosterol sulfate A, we first singled out the 5 best conformations that have the lowest $Q$ factors against a subgroup of RDC data. This subgroup of RDCs contains only the ${ }^{1} D_{\mathrm{CH}}$ couplings from the steroid ABCD and the linker part. Subsequently we determined the relative orientation of different conformers in one ensemble by superimposing each structure on steroid ABCD. The complete set of the RDC data was fitted on an ensemble of conformations and the selection of the correct configuration was performed based on the goodness of the RDC fitting. NOEs and ${ }^{3} J_{\mathrm{CH}}$ data were utilized as the additional data for the cross-validations. The schematic representation of the entire analysis 
Table 6.11: The conformation of the $\mathrm{C} 24-\mathrm{C} 25$ dihedral together with the populations of the three conformers determined by single-tensor analysis for the configuration $S S S$ and $S R R$.

\begin{tabular}{|c|c|c|c|c|c|}
\hline Configuration & Conformer & $\mathrm{H} 24-\mathrm{C} 26$ & $\mathrm{H} 24-\mathrm{C} 27$ & H24-C24' & Population \\
\hline \multirow[t]{3}{*}{$S S S$} & 1 & $\begin{array}{l}-170.6^{\circ} \\
(\text { trans })\end{array}$ & $\begin{array}{l}-49.5^{\circ} \\
(\text { gauche })\end{array}$ & $\begin{array}{c}62.4^{\circ} \\
\text { (gauche) }\end{array}$ & 0.26 \\
\hline & 2 & $\begin{array}{c}52.0^{\circ} \\
\text { (gauche) }\end{array}$ & $\begin{array}{l}-173.6^{\circ} \\
(\text { trans })\end{array}$ & $\begin{array}{c}72.7^{\circ} \\
\text { (gauche) }\end{array}$ & 0.45 \\
\hline & 3 & $\begin{array}{l}-52.3^{\circ} \\
\text { (gauche) }\end{array}$ & $\begin{array}{c}64.3^{\circ} \\
\text { (gauche) }\end{array}$ & $\begin{array}{l}-179.5^{\circ} \\
(\text { trans })\end{array}$ & 0.29 \\
\hline \multirow[t]{3}{*}{$S R R$} & 1 & $\begin{array}{l}-172.5^{\circ} \\
(\text { trans })\end{array}$ & $\begin{array}{c}64.8^{\circ} \\
\text { (gauche) }\end{array}$ & $\begin{array}{l}-47.7^{\circ} \\
\text { (gauche) }\end{array}$ & 0.59 \\
\hline & 2 & $\begin{array}{c}157.1^{\circ} \\
(\text { trans })\end{array}$ & $\begin{array}{c}37.1^{\circ} \\
(\text { gauche) }\end{array}$ & $\begin{array}{l}-71.5^{\circ} \\
\text { (gauche) }\end{array}$ & 0.13 \\
\hline & 3 & $\begin{array}{l}-179.9^{\circ} \\
(\text { trans })\end{array}$ & $\begin{array}{c}58.8^{\circ} \\
\text { (gauche) }\end{array}$ & $\begin{array}{l}-52.9^{\circ} \\
\text { (gauche) }\end{array}$ & 0.28 \\
\hline
\end{tabular}

procedure is shown in Figure 6.11. Using the other steroid A'B'C'D' gives the same answer but with slightly higher $Q$ values.

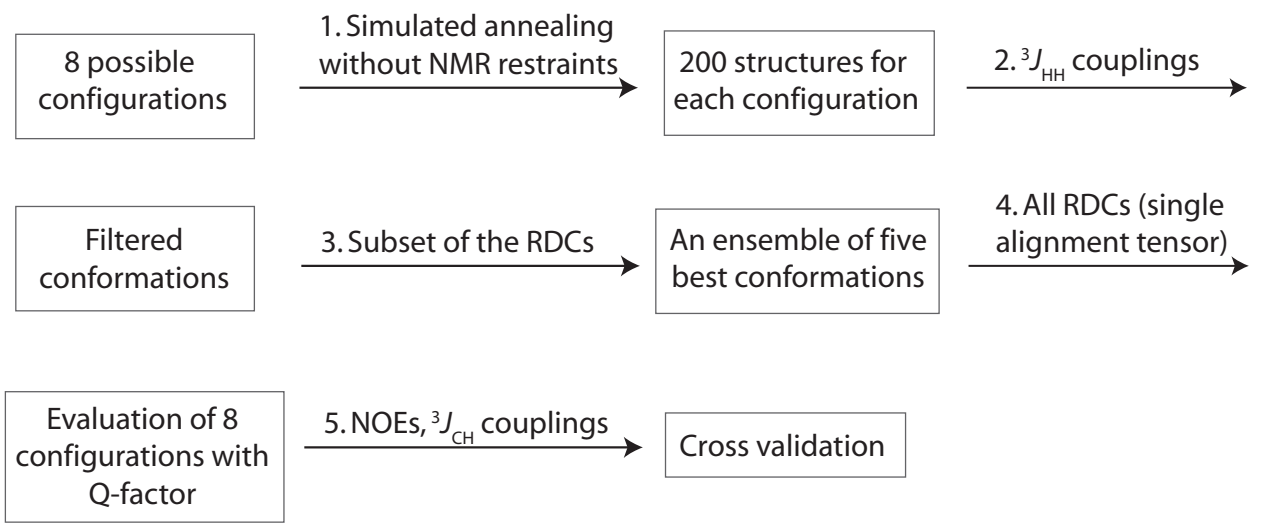

Figure 6.11: Schematic representation of the RDC-based single-tensor analysis used for configurational and conformational studies of fibrosterol sulfate A. 


\subsubsection{Comparison of the RDC-based single-tensor analysis and multiple-tensors analysis.}

The two different RDC-based approaches presented here independently led to the same configuration $S S S$ of the three unknown stereocenters of fibrosterol sulfate A, and similar conformer distribution. A remarkable difference between the two approaches lies in the fact, that in the single-tensor approach, the populations could be calculated directly using RDC data on a conformational ensemble obtained by a simple simulated annealing computation. However this requires the unphysical assumption that the tensors are the same for all conformations (Figure 6.10 and Table 6.9). By contrast, in the multiple-tensors fitting, since the magnitude of the order tensors and the populations are linearly dependent, information about the populations of the conformations or the relative sizes of the tensors are required. Here, we derived the populations from an MD simulation and could then determine the magnitudes of the tensors. Thus, the single-tensor approach is simpler and less time consuming. However the single alignment tensor assumption is physically implausible because conformers in one ensemble will have different alignment tensors. It is of interest to note, that for fibrosterol sulfate A, although the populated conformers have a very divergent shape, the single-tensor fitting gives the same result as the multiple-tensors fitting approach. This may be due to the fact that the major contribution to the dipolar coupling comes from the extended conformer (conformer 2). According to the MD and multiple-tensors analysis this conformer has the biggest population (48\%) and a considerably larger magnitude of the tensor than the other conformations. It is interesting to explore the limits of the single alignment tensor approach because it is much simpler than the multiple tensors approach and the result here seems to indicate that the determination of the configuration is pretty robust even if the shape of the molecule dramatically varies.

It is also interesting to note that using two different approaches the second best-fitting configuration is not the same. The second best configuration from the multiple-tensors approach was determined as $R S R$, whereas the one from the singletensor approach is $S R R$. The reason for this surprising behavior might be that we included isotropic NMR parameters, such as NOEs and ${ }^{3} J$ couplings as additional structural restraints at different stages of the analysis in the single alignment tensor and in the multiple alignment tensors approach. In the first approach, ${ }^{3} J_{\mathrm{HH}} \mathrm{cou}-$ plings were initially used as a filter to select a reasonably good ensemble of structures for RDC analysis. Finally, when NOEs and ${ }^{3} J_{\mathrm{CH}}$ couplings were adopted to crossvalidate the ensemble determined by RDC fitting, the ensemble of the configuration $S R R$ shows a very poor agreement with the experimental ${ }^{3} J_{\mathrm{CH}}$ couplings. However, 
in the multiple-tensors approach, the complete set of ${ }^{3} J$ couplings and NOEs was used to select a part of MD trajectory from the $100 \mathrm{~ns}$ simulation that fulfills the experimental data best. Therefore the determined ensembles of structures for the rejected configurations, which cannot fulfill NOEs, RDCs and ${ }^{3} J$ couplings simultaneously, will be different in both approaches.

\subsubsection{Validation of C20 and C20' configuration using RDC data}

The natural $R$ configuration at C20 and C20' was proposed previously on the basis of biogenic principles [Whitson et al., 2009]. Here, we validated the configuration of these two stereocenters using the experimental RDC data. The determined configuration $S S S$, shown in Figure 6.5 has an $R$ configuration at both C20 and C20' stereocenters. We generated two other configurations, turning C20 and C20' into $S$. To predict the RDCs of C20-H20 and C21-H21 for each conformer, we determined the alignment tensor by using the RDCs from the steroid ABCD. The averaged ${ }^{1} D_{\mathrm{CH}}$ couplings (C20-H20, C21-H21) for both possible configurations C20- $R$ or $S$ were calculated on the basis of the population from the MD simulations given in Table 6.5 and are listed in Table 6.12. Similarly, the RDC of C21'-H21' for C20'- $R$ or $S$ was predicted from the tensor, determined by using the RDCs from A'B'C'D'. The experimental RDC of C20'-H20' can not be extracted accurately, therefore it has not be used in the analysis here. Comparing the predicted RDCs of the two possible configurations shown in Table 6.12, it is obvious that the configuration with an $R$ configuration at $\mathrm{C} 20$ and $\mathrm{C} 20$ ' has a much better agreement with the experimental data, which further confirmed the configuration proposed by the biogenic principles.

\subsubsection{Conclusion}

Fibrosterol sulfate A is a polysulfated bis-steroid with an atypical side chain. Due to the flexibility of the linker, a large-scale motion that changes dramatically the shape of the entire molecule is expected. Such motions pose major challenges to the structure elucidation and the correct determination of configuration. In this study we have developed two different approaches for studying the relative configuration and conformation of fibrosterol sulfate A. The three previously unknown stereocenters C22, C25 and C24' were all determined to have $S$ configuration, and the molecule exists in solution as a mixture of the extended, closed and L-shaped con- 
Table 6.12: The predicted RDCs of C20-H20, C21-H21 and C21'-H21' for each conformer of SSS (Figure 6.3) and for the conformers, in which the stereochemistry of C20 and C20' were inverted. The alignment tensors were determined by using the RDCs from steroid $\mathrm{ABCD}$ for the RDCs of the unprimed $\mathrm{CH}$ pairs and A'B'C'D' for the RDCs of the primed ones, respectively.

\begin{tabular}{lllcrrrr}
\hline & & \multicolumn{7}{c}{${ }^{1} D_{\mathrm{CH}}(\mathrm{Hz})$} \\
& & Conf. 1 & Conf. 2 & Conf. 3 & Conf. 4 & Aver. & Exp. \\
\hline C20 $R$ & C20-H20 & 14.2 & 22.2 & -14.3 & 2.4 & 14.3 & 19.7 \\
C20' $R$ & C21-H21 & 3.7 & 5.4 & -0.7 & 2.7 & 4.0 & 2.2 \\
& C21'-H21' & 0.2 & 0.8 & -2.1 & -5.0 & -0.2 & 0.2 \\
\hline C20 $S$ & C20-H20 & -2.1 & 0.7 & -9.9 & -10.1 & -2.3 & 19.7 \\
C20' $S$ & C21-H21 & -3.4 & -2.9 & 1.4 & -2.9 & -2.7 & 2.2 \\
& C21'-H21' & -8.0 & -6.2 & 2.4 & 2.7 & -5.1 & 0.2 \\
\hline
\end{tabular}

formations. This finding demonstrates the power of RDCs in the determination of configuration and conformation of flexible organic molecules, which in many cases cannot be achieved by using conventional isotropic NMR parameters in solution. Both the single and the multiple tensors approaches yielded the same result, despite the large shape change between the different conformations. The multiple-tensor approach fits many more parameters to the experimental data, requiring a sufficient number of accurate RDCs. In addition, the populations of the conformers needed to be determined independently, here from explicit-solvent MD simulations. Although the single-tensor approach leads to similar results, presumably by the fact that the major contribution to the RDCs comes from the more strongly aligned extended conformation, this molecule could be of the class of extreme cases, where the single alignment tensor assumption still works but is reliable only if the multiple alignment tensor approach yields the same result. 


\section{Chapter 7}

\section{Conclusions and outlook}

\subsection{Conclusions}

This thesis describes the stereochemical elucidation of seven challenging organic molecules, that were categorized into three classes: A (LLG1, vatiparol, naphth1 and naphth 2), B1 (a Michael addition product and comp.540a) and B2 (fibrosterol sulfate A), based on the flexibility and connectivity of all stereogenic centers. In all cases except naphth 1 and naphth 2, residual dipolar couplings have been shown to possess an irreplaceable role for determining the relative configurations and the accurate conformation. Conventional isotropic NMR parameters such as NOEs and ${ }^{2,3} J$-couplings have been extensively used in these studies as well, either as an independent parameter for the complete stereochemical assignment (for a Michael addition product and vatiparol), or as additional NMR restraints to narrow down the conformational or configurational possibilities (for LLG1, comp.540a and fibrosterol sulfate A). Although in the last several years ab initio calculations of chemical shifts and chemical shift anisotropies (RCSAs) have also been shown to be useful for the establishment of the constitution and configuration of medium-sized molecules [Di Micco et al., 2010, Bifulco et al., 2007], we have not explored the usage of chemical shift and residual chemical shift anisotropies in this thesis.

For the molecules of class A, since the stereogenic centers communicate with each other, the stereocenters can be determined in many cases by a comparison of the experimental ${ }^{2,3} \mathrm{~J}$-couplings and NOE data with the suggested structural models. If the isotropic NMR data is not sufficient for the determination, RDC fitting on a single conformer can be employed to select the correct configuration and conformation, as shown in the case of LLG1. For molecules of class B1 with multiple stereogenic domains, RDCs are even more powerful, because different from NOEs and $J$-couplings that are short-range in nature, they provide long-range structural 
information that is able to correlate distant parts within a molecule. In the presented thesis, this property of the RDCs was extensively used and the stereochemistry of three molecules of class B1 was successfully determined. Dealing with the conformational flexibility in the RDC analysis, if the shapes of the various conformations are similar, all conformations having the same alignment tensor is a valid assumption and also a good method of choice. There are two reasons for this: (i) the number of the tensor parameters that need to be determined in the single-tensor analysis is much smaller than in the case of the multi-tensors approach, which for many small organic molecules is beyond experimental possibilities; (ii) in the single-tensor analysis the population of the conformations can be determined together with the tensor data, whereas in the multiple-tensors analysis the conformational distribution needs to be determined from other sources. Because of these advantages, the single-tensor approach was exclusively applied for all three molecules of class B1. For fibrosterol sulfate A that belongs to the molecule class B2, elucidation of relative stereochemistry was very challenging, and thus single-tensor and multiple-tensors approaches were both employed and compared. In this study we found surprisingly that although the single-tensor approach relies on the incorrect assumption of the same alignment tensor for each conformation and is therefore unreliable, both methods yield the same relative configuration and a similar conformational distribution, despite the apparent large differences in molecular shape with different linker conformations. This result can be explained by the fact that the major contribution to the RDCs comes from the more strongly aligned extended conformation. Fibrosterol sulfate $\mathrm{A}$ is therefore an extreme example of molecule class B2 in that the singletensor analysis can still be properly utilized for the determination of configuration.

The relative stereochemistry of local segments can be determined in some cases by a comparison of experimental data with some simple structural models, for example the Newman projections. This method was demonstrated in our studies of LLG1, comp.540a and vatiparol. Nevertheless, from the structural calculation point of view, computational simulations and DFT-based optimizations were more essential for the stereochemical elucidation, as they were able to provide direct and accurate structural images of those molecules, which are especially necessary for the RDC analysis. For relatively rigid molecules such as naphth 1, naphth 2 and vatiparol, or molecules with a reduced conformational space by experimental restraints such as LLG1, we used exclusively a combination of the molecular mechanics based conformational search and DFT optimizations to get a single structure or an ensemble of structures. Structure optimizations with DFT for small to medium-sized molecules are highly useful, as the obtained structures have a much better quality 
than the energy-minimized structures from the molecular mechanics based calculations. Here we used the hybrid functional B3LYP in combination with the basis sets 6-31G(d) or 6-311G(2d,p), which are commonly used versions for organic compounds. For flexible molecules with large conformational space, molecular dynamics simulations are a powerful sampling technique, which was demonstrated in the studies of a Michael addition product, comp.540a and fibrosterol sulfate A. Furthermore, in cases where the conformational distribution needed to be determined from the simulations, molecular dynamics simulations were more advantageous than simulated annealing or molecular mechanics based conformational search, as the population of the main conformers can be calculated from the trajectories. However, on the other hand, if the energy barriers between two conformers are relatively high, simulated annealing computations or Monte-Carlo based simulations are better methods of choice for a more extensive sampling of conformations.

In this thesis, the usage of specifically adapted TCI-cryoprobes with a diameter of $1.7 \mathrm{~mm}$ that became commercially available in 2007 [Molinski, 2010b], has been investigated. For the RDC measurements, a gel apparatus with an inner diameter of $2 \mathrm{~mm}$ has been accordingly adjusted to the $1.7 \mathrm{~mm}$ NMR tubes for the preparation of PH-gels (Figure 3.1). The swelling time of the dried gels in the $1.7 \mathrm{~mm}$ tubes is comparable or slightly longer than of the ones in $5 \mathrm{~mm}$ NMR tubes. The sensitivity based on the same amount of sample was determined to be 3 to 5 times higher for the $1.7 \mathrm{~mm}$ probe than for the the conventional $5 \mathrm{~mm}$ probe working at the same spectrometer. Additionally, much sharper resonance lines than for anisotropic samples run in $5 \mathrm{~mm}$ tubes has been noted. This effect might be due to easier shimming in the tube with the smaller diameter, but could also arise from the more homogeneous gel in the smaller volume. It should be noted that without the development of the PH-gels in the $1.7 \mathrm{~mm}$ NMR tube, RDC measurements and stereochemical elucidations of three rare natural products in the presented thesis, which are vatiparol, comp.540a and LLG1, would not have been possible. Furthermore, another polyacrylamide based $(R)$-APhES-gel (also for $1.7 \mathrm{~mm}$ NMR tubes) was tested together with the PH-gel on comp.540a, where they yielded alignment tensors with very different orientations in the five-dimensional space, which allows to obtain independent RDC data sets.

Since the stereochemical possibilities scale with $2^{n}$ where $\mathrm{n}$ is the number of stereocenters, stereochemical elucidation of natural products containing a large number of stereocenters can be highly time-consuming. Especially when RDC analysis is part of the configurational determination, cross-validation of each possible stereoiso- 
mer against the RDC data is in many cases a procedure that exceeds the technical possibilities. To solve this problem, one generally utilized method is reducing the configurational possibilities in the first step using ${ }^{2,3} J$-coupling and NOE analysis or based on some geometrical considerations. This approach has been employed in the configurational determination of a Michael addition product and vatiparol. In this thesis, restrained MD simulations with the floating chirality method that allow a simultaneous determination of the relative configuration, has been explored as well and demonstrated in the study of comp.540a. Here it was shown that when NOEs and $J$-couplings were used as restraints in the simulations, the large number of possible configurations can be significantly reduced and the final configuration was then determined by a cross-validation against the RDC data. The fact that three restrained simulations with randomly selected starting configurations lead to the same relative configurations indicates the robustness of the introduced method.

With the recent progress in ab initio quantum mechanics computations of chiroptical properties, chiroptical techniques have proven to be important for the establishment of the absolute stereochemistry of chiral molecules. In our study, this approach has been continuously applied and further combined with RDC-enhanced NMR spectroscopy that enables the accurate determination of relative configuration and conformation. The combination of these different techniques allows the absolute stereochemical assignment of even highly flexible molecules, as shown in chapter 4 with the example of a Michael addition product. The most frequently used chiroptical methods in this study are optical rotatory dispersion (ORD) and electronic circular dichroism (ECD). From the experimental point of view, ORD is a straightforward method, however it requires 1 to $10 \mathrm{mg}$ of sample. In comparison, ECD has the immense advantage of high sensitivity, so that only sub-microgram amounts of material are needed, however it is only a method of choice if active chromophores are present. For the computations of ECD and ORD spectra, excited states of the molecules need to be calculated. Although computations of VCD spectra entail only the ground state information of the molecule, this technique has not been used in our study, due to the requirement of high sample quantities for the measurements rendering it an unsuitable method for the study of natural products with limited amount of material. In the presented work, chiroptical properties have been computed with time-dependent DFT calculations. Similarly to the structural optimizations, the hybrid functional B3LYP in combination with the basis set 6$31 \mathrm{G}(\mathrm{d})$ was applied. For the ECD calculations we found that the calculated spectra were in general red- or blue-shifted compared to the experimental ones, as shown in the studies of LLG1 and vatiparol. This is due to the fact that using the B3LYP 
functionals the excitation energy can be predicted either too high or too low, depending on which system class is considered. This observation was described in many other previous studies [Giorgio et al., 2005, Di Bari et al., 2005, Berova et al., 2007] and was systematically investigated in [Diedrich and Grimme, 2003]. Because of this shift problem, when the absolute configuration was determined by comparison of the experimental and computed ECD spectra, it was important to compare the whole spectrum rather than focusing on one single maximum or minimum.

Due to the fact that chiroptical properties of molecules do not only depend on the configuration but also on the overall molecular conformation, for the correct elucidation of the absolute configuration it is essential to determine the conformation and their distribution in the solution accurately. In this thesis we have studied the conformational distribution with very different ways. One method relies on the quantum mechanics based energy calculation, and has been utilized in our study of naphth 1 and naphth 2. However, in many cases the energy of the systems can not be computed accurately using conventional DFT calculations, as illustrated in [Wodrich et al., 2007] and also shown in our study of LLG1. Therefore, although this quantum-mechanics based approach does not require any prior knowledge from the experimental side, it is only a method of choice if the chiroptical properties have a low dependence on the accuracy of the computed populations. Better approaches for determining the conformational distribution use experimental evidence such as NMR data. As mentioned above, if the shape of the different molecular conformations is similar, populations of the conformations together with five independent tensor parameters can be directly calculated from the RDC-based single-tensor analysis. This method has been employed in the studies of comp.540a and vatiparol. For a Michael addition product, a similar approach was applied. In this study, a large pool of ensembles was generated for each possible configuration, where all conformations in one ensemble were assumed to have the same population. NOEs and RDCs were used independently to select the best ensemble with correct conformational distribution, which was reflected in this case by the number of similar conformations within one ensemble. Different from the single-tensor analysis, in the multiple-tensors analysis only the product of population and alignment tensor can be derived from the experimentally measured RDCs. The two unknown parameters cannot be separated unless additional information on either the population of each conformer in the ensemble or the relative sizes of the alignment tensors for each conformer is obtained from independent sources. In the study of fibrosterol sulfate A, the population of the main conformers was determined from a NOE and $J$-coupling selected MD trajectory using conformational clustering. Furthermore, comparing 
the properties of different NMR parameters, one should note that the measured scalar and dipolar couplings are linearly weighted values over the couplings of all conformers accessible on the NMR time scale. Therefore only the distribution of the main populated conformers can be determined reliably. For exploring the sparsely populated conformations, NOE is one of the most important parameters because of its minus six averaging property. However, it should also be pointed out that NOEs are averaged in a much more complicated way than RDCs, and therefore in the NOE analysis a large experimental error needs to be taken into account.

\subsection{Outlook}

In this study, a general approach on the basis of RDC-enhanced NMR analysis combined with computational calculations and chiroptical spectroscopy was proposed for the stereochemical determination of natural products from different molecule classes. The presented methodology should be continuously applied in further studies and tested on other challenging systems, which can not be determined with conventional methods. Especially for flexible molecules that are not crystallizable, we believe this integrated method has a great advantage for the absolute stereochemical elucidation.

In the presented thesis, long-range RDCs have not yet been employed in the structure determination due to two reasons: (i) Compared to one-bond ${ }^{1} \mathrm{H}^{13} \mathrm{C}$ RDCs, long-range RDCs have a relatively small magnitude and are thus difficult to be measured with high precision; (ii) most of the published NMR experiments for the measurement of long-range RDCs have a significantly lower sensitivity compared to the $t_{2^{-}}$or $t_{1}$-coupled HSQC experiments that are used for the one-bond ${ }^{1} \mathrm{H}_{-}{ }^{13} \mathrm{C}$ RDC measurements, which makes the application of such techniques to natural products with very limited amount of material challenging. Nevertheless, after the development of the alignment media compatible with the $1.7 \mathrm{~mm}$ NMR tubes (see chapter 5), together with a recently introduced pulse sequence for measuring longrange RDCs with high sensitivity and resolution [Trigo-Mouriño et al., 2011], the usage and advantage of long-range RDCs can be explored more extensively in further investigations. Especially in the multiple-tensors approach, where the number of the experimental RDCs required is much higher than in the case of the single-tensor analysis, long-range RDCs will show great potential in the stereochemical determination.

Besides the long-range RDCs, the usage of the residual chemical shift anisotropies should be more investigated as well in the future. In one previous study [Hallwass 
et al., 2011], it was shown with the example of estrone that RCSAs provide important orientation information that can be used to determine the configuration of molecules. For cases similar to estrone where the stereogenic centers have no or too few protons, RCSA could be the only source for the stereochemical elucidation. From the technical point of view, different methods for the measurement of the RCSA, for example using the stretching apparatus, variable-angle sample spinning, or alignment media with different concentrations that lead to different degrees of alignment, should be tested and compared. Computations of the CSA tensor using different strategies need to be evaluated as well. Furthermore, the use and interplay between isotropic NMR parameters, one-bond ${ }^{1} \mathrm{H}-{ }^{13} \mathrm{C}$ RDCs, long-range RDCs, and RCSAs in the conformational and configurational study is another important issue that should be investigated more extensively in furture work. 


\section{Bibliography}

[Allenmark and Gawronski, 2008] Allenmark, S. and Gawronski, J. (2008). Determination of absolute configuration - An overview related to this Special Issue. Chirality, 20(5):606-608.

[Almond and Axelsen, 2002] Almond, A. and Axelsen, J. B. (2002). Physical interpretation of residual dipolar couplings in neutral aligned media. J. Am. Chem. Soc., 124(34):9986-9987.

[Andrade, 2004] Andrade, C. (2004). Niobium pentachloride in organic synthesis: Applications and perspectives. Curr. Org. Synth., 1(4):333-353.

[Andrade and Rocha, 2006] Andrade, C. K. Z. and Rocha, R. O. (2006). Recent applications of niobium catalysts in organic synthesis. Mini-Rev. Org. Chem., $3(4): 271-280$.

[Arnold et al., 2010] Arnold, L., Marx, A., Thiele, C. M., and Reggelin, M. (2010). Polyguanidines as chiral orienting media for organic compounds. Chemistry, 16(34):10342-10346.

[Aue et al., 1976] Aue, W., Bartholdi, E., and Ernst, R. (1976). 2-dimensional spectroscopy - Application to nuclear magnetic-resonance. J. Chem. Phys., 64(5):2229-2246.

[Azurmendi and Bush, 2002] Azurmendi, H. F. and Bush, C. A. (2002). Tracking alignment from the moment of inertia tensor (TRAMITE) of biomolecules in neutral dilute liquid crystal solutions. J. Am. Chem. Soc., 124(11):2426-2427.

[Bach et al., 1995] Bach, J., Berenguer, R., Garcia, J., and Vilarrasa, J. (1995). A synthetic approach towards octalactin-A, based on the stereoselective reduction of alpha,beta-unsaturated ketones. Tetrahedron Lett., 36(19):3425-3428.

[Baur et al., 2006] Baur, J. A., Pearson, K. J., Price, N. L., Jamieson, H. A., Lerin, C., Kalra, A., Prabhu, V. V., Allard, J. S., Lopez-Lluch, G., Lewis, K., Pistell, P. J., Poosala, S., Becker, K. G., Boss, O., Gwinn, D., Wang, M., Ramaswamy, 
S., Fishbein, K. W., Spencer, R. G., Lakatta, E. G., Le Couteur, D., Shaw, R. J., Navas, P., Puigserver, P., Ingram, D. K., de Cabo, R., and Sinclair, D. A. (2006). Resveratrol improves health and survival of mice on a high-calorie diet. Nature, 444(7117):337-342.

[Bax and Davis, 1985] Bax, A. and Davis, D. (1985). MLEV-17-based twodimensional homonuclear magnetization transfer spectroscopy. J. Magn. Reson., 65(2):355-360.

[Bax and Summers, 1986] Bax, A. and Summers, M. (1986). H-1 and C-13 assignments from sensitivity-enhanced detection of heteronuclear multiple-bond connectivity by 2D multiple quantum NMR. J. Am. Chem. Soc., 108(8):2093-2094.

[Bernadó et al., 2005a] Bernadó, P., Bertoncini, C. W., Griesinger, C., Zweckstetter, M., and Blackledge, M. (2005a). Defining long-range order and local disorder in native alpha-synuclein using residual dipolar couplings. J. Am. Chem. Soc., 127(51):17968-17969.

[Bernadó et al., 2005b] Bernadó, P., Blanchard, L., Timmins, P., Marion, D., Ruigrok, R. W., and Blackledge, M. (2005b). A structural model for unfolded proteins from residual dipolar couplings and small-angle X-ray scattering. Proc. Natl. Acad. Sci. U. S. A., 102(47):17002-17007.

[Berova et al., 2007] Berova, N., Di Bari, L., and Pescitelli, G. (2007). Application of electronic circular dichroism in configurational and conformational analysis of organic compounds. Chem. Soc. Rev., 36(6):914-931.

[Bifulco et al., 2007] Bifulco, G., Dambruoso, P., Gomez-Paloma, L., and Riccio, R. (2007). Determination of relative configuration in organic compounds by NMR spectroscopy and computational methods. Chem. Rev., 107(9):3744-3779.

[Bijvoet et al., 1951] Bijvoet, J. M., Peerdeman, A. F., and Van Bommel, A. J. (1951). Determination of the absolute configuration of optically active compounds by means of X-rays. Nature, 168(4268):271-272.

[Blackledge, 2005] Blackledge, M. (2005). Recent progress in the study of biomolecular structure and dynamics in solution from residual dipolar couplings. Prog. Nucl. Magn. Reson. Spectrosc., 46(1):23-61.

[Bloch et al., 1946] Bloch, F., Hansen, W. W., and Packard, M. (1946). Nuclear induction. Phys. Rev., 69(3-4):127. 
[Bodenhausen and Ruben, 1980] Bodenhausen, G. and Ruben, D. (1980). Natural abundance N-15 NMR by enhanced heteronuclear spectroscopy. Chem. Phys. Lett., 69(1):185-189.

[Bothner-By et al., 1984] Bothner-By, A., Stephens, R., Lee, J., Warren, C., and Jeanloz, R. (1984). Structure determination of a tetrasaccharide: transient nuclear overhauser effects in the rotating frame. J. Am. Chem. Soc., 106(3):811-813.

[Braunschweiler and Ernst, 1983] Braunschweiler, L. and Ernst, R. (1983). Coherence transfter by isotropic mixing - application to proton correlation spectroscopy. J. Magn. Reson., 53(3):521-528.

[Burnell and Delange, 1980] Burnell, E. and Delange, C. (1980). On the average orientation of molecules undergoing large-amplitude conformational-changes in anisotropic liquids. Chem. Phys. Lett., 76(2):268-272.

[Catalano et al., 1991] Catalano, D., Dibari, L., Veracini, C., Shilstone, G., and Zannoni, C. (1991). A maximum entropy analysis of the problem of the rotameric distribution for substituted biphenyls studied by H-1 nuclear-magnetic-resonance spectroscopy in nematic liquid-crystals. J. Chem. Phys., 94(5):3928-3935.

[Cavanagh et al., 2006] Cavanagh, J., Fairbrother, W. J., Palmer, A. G., Skelton, N. J., and Rance, M. (2006). Protein NMR Spectroscopy. Principles and practice. Academic Press, 2 rev. edition.

[Cavanagh and Rance, 1990] Cavanagh, J. and Rance, M. (1990). Sensitivity improvement in isotropic mixing (TOCSY) experiments. J. Magn. Reson., 88(1):7285.

[Choi et al., 2011] Choi, C. W., Choi, Y. H., Cha, M.-R., Kim, Y. S., Yon, G. H., Hong, K. S., Park, W.-K., Kim, Y. H., and Ryu, S. Y. (2011). In vitro BACE1 inhibitory activity of resveratrol oligomers from the seed extract of paeonia lactiflora. Planta Med., 77(4):374-376.

[Christen et al., 2005] Christen, M., Hünenberger, P. H., Bakowies, D., Baron, R., Bürgi, R., Geerke, D. P., Heinz, T. N., Kastenholz, M. A., Kräutler, V., Oostenbrink, C., Peter, C., Trzesniak, D., and van Gunsteren, W. F. (2005). The GROMOS software for biomolecular simulation: GROMOS05. J. Comput. Chem., 26(16):1719-1751.

[Chung et al., 2003] Chung, E., Kim, B., Lee, M., Yun, Y., Lee, S., Min, K., and Kim, Y. (2003). Anti-inflammatory effect of the oligomeric stilbene alpha-viniferin 
and its mode of the action through inhibition of cyclooxygenase-2 and inducible nitric oxide synthase. Planta Med., 69(8):710-714.

[Cicero et al., 2001] Cicero, D. O., Barbato, G., and Bazzo, R. (2001). Sensitivity enhancement of a two-dimensional experiment for the measurement of heteronuclear long-range coupling constants, by a new scheme of coherence selection by gradients. J. Magn. Reson., 148(1):209-213.

[Cohen et al., 2006] Cohen, E., Lingen, M., Zhu, B., Zhu, H., Straza, M., Pierce, C., Martin, L., and Rosner, M. (2006). Protein kinase C mediates epidermal growth factor-induced growth of head and neck tumor cells by regulating mitogenactivated protein kinase. Cancer Res., 66(12):6296-6303.

[Cornilescu et al., 1998] Cornilescu, G., Marquardt, J., Ottiger, M., and Bax, A. (1998). Validation of protein structure from anisotropic carbonyl chemical shifts in a dilute liquid crystalline phase. J. Am. Chem. Soc., 120(27):6836-6837.

[Cragg et al., 2009] Cragg, G. M., Grothaus, P. G., and Newman, D. J. (2009). Impact of natural products on developing new anti-cancer agents. Chem. Rev., 109(7):3012-3043.

[Cyr et al., 1978] Cyr, N., Hamer, G. K., and Perlin, A. S. (1978). Some stereochemical aspects of two-bond 13C-1H spin-spin coupling. Sign determination in natural abundance 13C nuclear magnetic resonance spectra. Can. J. Chem, 56:297-301.

[Dalisay and Molinski, 2010] Dalisay, D. S. and Molinski, T. F. (2010). Structure elucidation at the nanomole scale. 3. Phorbasides G-I from Phorbas sp. J. Nat. Prod., 73(4):679-682.

[d'Auvergne and Gooley, 2008a] d'Auvergne, E. J. and Gooley, P. R. (2008a). Optimisation of NMR dynamic models I. Minimisation algorithms and their performance within the model-free and Brownian rotational diffusion spaces. J. Biomol. NMR, 40(2):107-119.

[d'Auvergne and Gooley, 2008b] d'Auvergne, E. J. and Gooley, P. R. (2008b). Optimisation of NMR dynamic models II. A new methodology for the dual optimisation of the model-free parameters and the Brownian rotational diffusion tensor. J. Biomol. NMR, 40(2):121-133.

[Di Bari et al., 2005] Di Bari, L., Guillarme, S., Hermitage, S., Jay, D. A., Pescitelli, G., and Whiting, A. (2005). Absolute stereochemistry assignment of Nphosphorylimine-derived aza-Diels-Alder adducts with TDDFT CD calculations. Chirality, 17(6):323-331. 
[Di Micco et al., 2010] Di Micco, S., Chini, M. G., Riccio, R., and Bifulco, G. (2010). Quantum mechanical calculation of NMR parameters in the stereostructural determination of natural products. Eur. J. Org. Chem., (8):1411-1434.

[Diedrich and Grimme, 2003] Diedrich, C. and Grimme, S. (2003). Systematic investigation of modern quantum chemical methods to predict electronic circular dichroism spectra. J. Phys. Chem A., 107:2524-2539.

[Eliel et al., 2005] Eliel, E. L., Wilen, S. H., and P., D. M. (2005). Basic organic stereochemistry. John Wiley \& Sons, 1. edition.

[Emsley et al., 1982] Emsley, J., Luckhurst, G., and Stockley, C. (1982). A Theory of orientational ordering in uniaxial liquid-crystals composed of molecules with alkyl chains. Proc. R. Soc. London Ser. A-Math. Phys. Eng. Sci., 381(1780):117138.

[Enthart et al., 2008] Enthart, A., Freudenberger, J. C., Furrer, J., Kessler, H., and Luy, B. (2008). The CLIP/CLAP-HSQC: pure absorptive spectra for the measurement of one-bond couplings. J. Magn. Reson., 192(2):314-322.

[Ernst et al., 2004] Ernst, R. R., Bodenhausen, G., and Wokaun, A. (2004). Principles of nuclear magnetic resonance in one and two dimensions. Oxford University Press, reprinted edition.

[Evans et al., 1991] Evans, D., Bilodeau, M., Somers, T., Clardy, J., Cherry, D., and Kato, Y. (1991). Enantioselective Michael reactions - diastereoselective reactions of chlorotitanium enolates of chiral N-acyloxazolidinones with representative electrophilic olefins. J. Org. Chem., 56(20):5750-5752.

[Evans et al., 1990] Evans, D., Urpi, F., Somers, T., Clark, J., and Bilodeau, M. (1990). New procedure for the direct generation of titanium enolates - diastereoselective bond constructions with representative electrophiles. J. Am. Chem. Soc., 112(22):8215-8216.

[Freudenberger et al., 2005] Freudenberger, J. C., Knör, S., Kobzar, K., Heckmann, D., Paululat, T., Kessler, H., and Luy, B. (2005). Stretched poly(vinyl acetate) gels as NMR alignment media for the measurement of residual dipolar couplings in polar organic solvents. Angew. Chem. Int. Ed. Engl., 44(3):423-426.

[Freudenberger et al., 2004] Freudenberger, J. C., Spiteller, P., Bauer, R., Kessler, H., and Luy, B. (2004). Stretched poly(dimethylsiloxane) gels as NMR alignment media for apolar and weakly polar organic solvents: an ideal tool for measuring RDCs at low molecular concentrations. J. Am. Chem. Soc., 126(45):14690-14691. 
[Frisch et al., a] Frisch, M. J., Trucks, G. W., Schlegel, H. B., Scuseria, G. E., Robb, M. A., Cheeseman, J. R., Montgomery, Jr., J. A., Vreven, T., Kudin, K. N., Burant, J. C., Millam, J. M., Iyengar, S. S., Tomasi, J., Barone, V., Mennucci, B., Cossi, M., Scalmani, G., Rega, N., Petersson, G. A., Nakatsuji, H., Hada, M., Ehara, M., Toyota, K., Fukuda, R., Hasegawa, J., Ishida, M., Nakajima, T., Honda, Y., Kitao, O., Nakai, H., Klene, M., Li, X., Knox, J. E., Hratchian, H. P., Cross, J. B., Bakken, V., Adamo, C., Jaramillo, J., Gomperts, R., Stratmann, R. E., Yazyev, O., Austin, A. J., Cammi, R., Pomelli, C., Ochterski, J. W., Ayala, P. Y., Morokuma, K., Voth, G. A., Salvador, P., Dannenberg, J. J., Zakrzewski, V. G., Dapprich, S., Daniels, A. D., Strain, M. C., Farkas, O., Malick, D. K., Rabuck, A. D., Raghavachari, K., Foresman, J. B., Ortiz, J. V., Cui, Q., Baboul, A. G., Clifford, S., Cioslowski, J., Stefanov, B. B., Liu, G., Liashenko, A., Piskorz, P., Komaromi, I., Martin, R. L., Fox, D. J., Keith, T., Al-Laham, M. A., Peng, C. Y., Nanayakkara, A., Challacombe, M., Gill, P. M. W., Johnson, B., Chen, W., Wong, M. W., Gonzalez, C., and Pople, J. A. Gaussian 03, Revision C.02. Gaussian, Inc., Wallingford, CT, 2004.

[Frisch et al., b] Frisch, M. J., Trucks, G. W., Schlegel, H. B., Scuseria, G. E., Robb, M. A., Cheeseman, J. R., Scalmani, G., Barone, V., Mennucci, B., Petersson, G. A., Nakatsuji, H., Caricato, M., Li, X., Hratchian, H. P., Izmaylov, A. F., Bloino, J., Zheng, G., Sonnenberg, J. L., Hada, M., Ehara, M., Toyota, K., Fukuda, R., Hasegawa, J., Ishida, M., Nakajima, T., Honda, Y., Kitao, O., Nakai, H., Vreven, T., Montgomery, Jr., J. A., Peralta, J. E., Ogliaro, F., Bearpark, M., Heyd, J. J., Brothers, E., Kudin, K. N., Staroverov, V. N., Kobayashi, R., Normand, J., Raghavachari, K., Rendell, A., Burant, J. C., Iyengar, S. S., Tomasi, J., Cossi, M., Rega, N., Millam, J. M., Klene, M., Knox, J. E., Cross, J. B., Bakken, V., Adamo, C., Jaramillo, J., Gomperts, R., Stratmann, R. E., Yazyev, O., Austin, A. J., Cammi, R., Pomelli, C., Ochterski, J. W., Martin, R. L., Morokuma, K., Zakrzewski, V. G., Voth, G. A., Salvador, P., Dannenberg, J. J., Dapprich, S., Daniels, A. D., Farkas, ...., Foresman, J. B., Ortiz, J. V., Cioslowski, J., and Fox, D. J. Gaussian 09 Revision A.1. Gaussian Inc. Wallingford CT 2009.

[García et al., 2009] García, M. E., Pagola, S., Navarro-Vázquez, A., Phillips, D. D., Gayathri, C., Krakauer, H., Stephens, P. W., Nicotra, V. E., and Gil, R. R. (2009). Stereochemistry determination by powder X-ray diffraction analysis and NMR spectroscopy residual dipolar couplings. Angew. Chem. Int. Ed. Engl., 48(31):5670-5674.

[Gayathri et al., 2010] Gayathri, C., de la Fuente, M. C., Luy, B., Gil, R. R., and Navarro-Vázquez, A. (2010). Probing heterocycle conformation with residual 
dipolar couplings. Chem. Commun. (Camb.), 46(32):5879-5881.

[Ge et al., 2012] Ge, H. M., Sun, H., Jiang, N., Qin, Y. H., Dou, H., Yan, T., Hou, Y. Y., Griesinger, C., and Tan, R. X. (2012). Relative and absolute configuration of vatiparol $(1 \mathrm{mg})$ : a novel anti-inflammatory polyphenol. Chemistry, 18(17):5213-5221.

[Ge et al., 2010] Ge, H. M., Yang, W. H., Shen, Y., Jiang, N., Guo, Z. K., Luo, Q., Xu, Q., Ma, J., and Tan, R. X. (2010). Immunosuppressive resveratrol aneuploids from Hopea chinensis. Chemistry, 16(21):6338-6345.

[Ge et al., 2008] Ge, H. M., Zhu, C. H., Shi, d. a. H., Zhang, L. D., Xie, D. Q., Yang, J., Ng, S. W., and Tan, R. X. (2008). Hopeahainol A: an acetylcholinesterase inhibitor from Hopea hainanensis. Chemistry, 14(1):376-381.

[Gil et al., 2008] Gil, R. R., Gayathri, C., Tsarevsky, N. V., and Matyjaszewski, K. (2008). Stretched poly(methyl methacrylate) gel aligns small organic molecules in chloroform. stereochemical analysis and diastereotopic proton NMR assignment in ludartin using residual dipolar couplings and 3J coupling constant analysis. $J$. Org. Chem., 73(3):840-848.

[Giorgio et al., 2005] Giorgio, E., Roje, M., Tanaka, K., Hamersak, Z., Sunjic, V., Nakanishi, K., Rosini, C., and Berova, N. (2005). Determination of the absolute configuration of flexible molecules by ab initio ORD calculations: a case study with cytoxazones and isocytoxazones. J. Org. Chem., 70(17):6557-6563.

[Grant, 2007] Grant, D. M. (2007). Chemical shift tensors, Encyclopedia of Magnetic Resonance. John Wiley \& Sons, Ltd.

[Griesinger et al., 1985] Griesinger, C., Sorensen, O., and Ernst, R. (1985). Twodimensional correlation of connected NMR transitions. J. Am. Chem. Soc., 107(22):6394-6396.

[Griesinger et al., 1987] Griesinger, C., Sorensen, O., and Ernst, R. (1987). Practical aspects of the E.COSY technique. Measurement of scalar spin-spin coupling constants in peptides. J. Magn. Reson., 75(3):474-492.

[Grube and Köck, 2007] Grube, A. and Köck, M. (2007). Structural assignment of tetrabromostyloguanidine: does the relative configuration of the palau'amines need revision? Angew. Chem. Int. Ed. Engl., 46(13):2320-2324.

[Gu et al., 2000] Gu, L., Tseng, S., Horner, R. M., Tam, C., Loda, M., and Rollins, B. J. (2000). Control of th2 polarization by the chemokine monocyte chemoattractant protein-1. Nature, 404(6776):407-411. 
[Guo et al., 2009] Guo, H., Ma, Y., Zhang, B., Sun, B., Niu, R., Ying, G., and Zhang, N. (2009). Pivotal Advance: PKC zeta is required for migration of macrophages. J. Leukoc. Biol., 85(6):911-918.

[Haasnoot et al., 1980] Haasnoot, C. A. G., De Leeuw, F. A. A. M., and Altona, C. (1980). The relationship between proton-proton NMR coupling-constants and substituent electronegativities .1. an empirical generalization of the Karplus equation. Tetrahedron, 36(19):2783-2792.

[Haberz et al., 2005] Haberz, P., Farjon, J., and Griesinger, C. (2005). A DMSOcompatible orienting medium: Towards the investigation of the stereochemistry of natural products. Angew. Chem. Int. Ed. Engl., 44(3):427-429.

[Hagler et al., 1974] Hagler, A., Huler, E., and Lifson, S. (1974). Energy functions for peptides and proteins. 1. Derivation of a consistent force-field including hydrogen-bond from amide crystals. J. Am. Chem. Soc., 96(17):5319-5327.

[Hagler et al., 1979] Hagler, A., Lifson, S., and Dauber, P. (1979). Consistent forcefield studies of inter-molecular forces in hydrogen-bonded crystals. 2. Benchmark for the objective comparison of alternative force-fields. J. Am. Chem. Soc., 101(18):5122-5130.

[Hallwass et al., 2011] Hallwass, F., Schmidt, M., Sun, H., Mazur, A., Kummerlöwe, G., Luy, B., Navarro-Vázquez, A., Griesinger, C., and Reinscheid, U. M. (2011). Residual chemical shift anisotropy (RCSA): A tool for the analysis of the configuration of small molecules. Angew. Chem. Int. Ed. Engl., 50(40):9487-9490.

[Hansen and Hünenberger, 2011] Hansen, H. S. and Hünenberger, P. H. (2011). A reoptimized GROMOS force field for hexopyranose-based carbohydrates accounting for the relative free energies of ring conformers, anomers, epimers, hydroxymethyl rotamers, and glycosidic linkage conformers. J. Comput. Chem., 32(6):998-1032.

[Hanson, 2003] Hanson, J. R. (2003). Natural products: the secondary metabolites. Royal Society of Chemistry, 1 . edition.

[He et al., 2008] He, S., Wu, B., Pan, Y., and Jiang, L. (2008). Stilbene oligomers from Parthenocissus laetevirens: Isolation, biomimetic synthesis, absolute configuration, and implication of antioxidative defense system in the plant. J. Org. Chem., 73(14):5233-5241.

[Hoffmann, 2000] Hoffmann, R. (2000). Conformation design of open-chain compounds. Angew. Chem. Int. Ed. Engl., 39(12):2054-2070. 
[Jang et al., 1997] Jang, M., Cai, L., Udeani, G. O., Slowing, K. V., Thomas, C. F., Beecher, C. W., Fong, H. H., Farnsworth, N. R., Kinghorn, A. D., Mehta, R. G., Moon, R. C., and Pezzuto, J. M. (1997). Cancer chemopreventive activity of resveratrol, a natural product derived from grapes. Science, 275(5297):218-220.

[Janoschek, 1991] Janoschek, R. (1991). Chirality - From weak bosons to the $\alpha$-helix. Springer-Verlag Berlin Heidelberg, 1. edition.

[Jeener et al., 1979] Jeener, J., Meier, B., Bachmann, P., and Ernst, R. (1979). Investigation of exchange processes by 2-dimensional NMR-spectroscopy. J. Chem. Phys., 71(11):4546-4553.

[Jha et al., 2005] Jha, A. K., Colubri, A., Freed, K. F., and Sosnick, T. R. (2005). Statistical coil model of the unfolded state: resolving the reconciliation problem. Proc. Natl. Acad. Sci. U. S. A., 102(37):13099-13104.

[Karplus, 1959] Karplus, M. (1959). Contact electron-spin coupling of nuclear magnetic moments. J. Chem. Phys., 30(1):11-15.

[Karplus, 1963] Karplus, M. (1963). Vicinal proton coupling in nuclear magnetic resonance. J. Am. Chem. Soc., 85(18):2870-2871.

[Kawahara et al., 2003] Kawahara, S., Kojima, C., Taira, K., and Uchimaru, T. (2003). A theoretical study of correlation between hydrogen-bond stability and J-coupling through a hydrogen bond. Helv. Chim. Acta, 86(10):3265-3273.

[Keeler, 2010] Keeler, J. (2010). Understanding NMR Spectroscopy. John Wiley \& Sons, Ltd, 2 ed. edition.

[Köck et al., 2012] Köck, M., Schmidt, G., Seiple, I. B., and Baran, P. S. (2012). Configurational analysis of tetracyclic dimeric pyrrole-imidazole alkaloids using a floating chirality approach. J. Nat. Prod., 75(2):127-130.

[Koradi et al., 1996] Koradi, R., Billeter, M., and Wuthrich, K. (1996). MOLMOL: A program for display and analysis of macromolecular structures. J. Mol. Graph., $14(1): 51-55$.

[Kozminski and Nanz, 1997] Kozminski, W. and Nanz, D. (1997). HECADE: HMQC- and HSQC-based 2D NMR experiments for accurate and sensitive determination of heteronuclear coupling constants from TOCSY-type cross peaks. J. Magn. Reson., 124(2):383-392. 
[Kramer et al., 2004] Kramer, F., Deshmukh, M., Kessler, H., and Glaser, S. (2004). Residual dipolar coupling constants: An elementary derivation of key equations. Concepts Magn. Reson. Part A, 21A(1):10-21.

[Kramer and Kleinpeter, 2011] Kramer, M. and Kleinpeter, E. (2011). A conformational study of $\mathrm{N}$-acetyl glucosamine derivatives utilizing residual dipolar couplings. J. Magn. Reson., 212(1):174-185.

[Kummerlöwe et al., 2007] Kummerlöwe, G., Auernheimer, J., Lendlein, A., and Luy, B. (2007). Stretched poly(acrylonitrile) as a scalable alignment medium for DMSO. J. Am. Chem. Soc., 129(19):6080-6081.

[Kummerlöwe et al., 2011] Kummerlöwe, G., Grage, S. L., Thiele, C. M., Kuprov, I., Ulrich, A. S., and Luy, B. (2011). Variable angle NMR spectroscopy and its application to the measurement of residual chemical shift anisotropy. J. Magn. Reson., 209(1):19-30.

[Kummerlöwe et al., 2009] Kummerlöwe, G., Kiran, M. U., and Luy, B. (2009). Covalently cross-linked gelatin allows chiral distinction at elevated temperatures and in DMSO. Chemistry, 15(45):12192-12195.

[Kummerlöwe and Luy, 2009] Kummerlöwe, G. and Luy, B. (2009). Residual dipolar couplings as a tool in determining the structure of organic molecules. TracTrends Anal. Chem., 28(4):483-493.

[Ladiwala et al., 2010] Ladiwala, A. R., Lin, J. C., Bale, S. S., Marcelino-Cruz, A. M., Bhattacharya, M., Dordick, J. S., and Tessier, P. M. (2010). Resveratrol selectively remodels soluble oligomers and fibrils of amyloid Abeta into off-pathway conformers. J. Biol. Chem., 285(31):24228-24237.

[Lawson and Hanson, 1974] Lawson, C. L. and Hanson, R. J. (1974). Solving Least Square Problems. Society for Industrial and Applied Mathematics, 1. edition.

[Lee et al., 2006] Lee, E.-O., Lee, H.-J., Hwang, H.-S., Ahn, K.-S., Chae, C., Kang, K.-S., Lu, J., and Kim, S.-H. (2006). Potent inhibition of Lewis lung cancer growth by heyneanol A from the roots of Vitis amurensis through apoptotic and anti-angiogenic activities. Carcinogenesis, 27(10):2059-2069.

[Li et al., 1994] Li, G., Patel, D., and Hruby, V. J. (1994). 1,2-Asymmetric cis induction and its application to the asymmetric synthesis of precursors of betabranched unusual amino acids". Tetrahedron Lett., 35(15):2301-2304. 
[Lifson and Warshel, 1968] Lifson, S. and Warshel, A. (1968). Consistent force field for calculations of conformations vibrational spectra and enthalpies of cycloalkane and n-alkane molecules. J. Chem. Phys., 49(11):5116-5129.

[Losonczi et al., 1999] Losonczi, J. A., Andrec, M., Fischer, M. W., and Prestegard, J. H. (1999). Order matrix analysis of residual dipolar couplings using singular value decomposition. J. Magn. Reson., 138(2):334-342.

[Lou et al., 1995] Lou, B.-S., Li, G., Lung, F.-D., and Hruby, V. J. (1995). NMR investigation of asymmetric conjugate additions using chiral 4-phenyloxazolidinone as a mechanistic probe. J. Org. Chem., 60(17):5509-5514.

[Luy et al., 2005] Luy, B., Kobzar, K., Knör, S., Furrer, J., Heckmann, D., and Kessler, H. (2005). Orientational properties of stretched polystyrene gels in organic solvents and the suppression of their residual $1 \mathrm{H}$ NMR signals. J. Am. Chem. Soc., 127(17):6459-6465.

[Mannhold et al., 2006] Mannhold, R., Kubinyi, H., and Folkers, G. (2006). Chirality in drug research. WILEY-VCH, 1. edition.

[Maple et al., 1988] Maple, J., Dinur, U., and Hagler, A. (1988). Derivation of forcefields for molecular mechanics and dynamics from ab initio energy surfaces. Proc. Natl. Acad. Sci. U. S. A., 85(15):5350-5354.

[Marx and Thiele, 2009] Marx, A. and Thiele, C. (2009). Orientational properties of poly-gamma-benzyl-L-glutamate: influence of molecular weight and solvent on order parameters of the solute. Chemistry, 15(1):254-260.

[McCann and Stephens, 2006] McCann, D. M. and Stephens, P. J. (2006). Determination of absolute configuration using density functional theory calculations of optical rotation and electronic circular dichroism: chiral alkenes. J. Org. Chem., 71(16):6074-6098.

[Molinski, 2009] Molinski, T. F. (2009). Nanomole-scale natural products discovery. Curr. Opin. Drug. Discov. Devel., 12(2):197-206.

[Molinski, 2010a] Molinski, T. F. (2010a). Microscale methodology for structure elucidation of natural products. Curr. Opin. Biotechnol., 21(6):819-826.

[Molinski, 2010b] Molinski, T. F. (2010b). Nmr of natural products at the 'nanomole-scale'. Nat. Prod. Rep., 27(3):321-329.

[Mori, 2011] Mori, K. (2011). Bioactive natural products and chirality. Chirality, 23(6):449-462. 
[Mueller, 1987] Mueller, L. (1987). PE-COSY, a simple alternative to E-COSY. J. Magn. Reson., 72(1):191-196.

[Mukaiyama et al., 1987] Mukaiyama, T., Iwasawa, N., Yura, T., and Clark, R. (1987). The tin(II) enolate addition-reactions to alpha,beta-unsaturated ketones and quinones. Tetrahedron, 43(21):5003-5017.

[Mukrasch et al., 2007] Mukrasch, M. D., Markwick, P., Biernat, J., Bergen, M., Bernadó, P., Griesinger, C., Mandelkow, E., Zweckstetter, M., and Blackledge, M. (2007). Highly populated turn conformations in natively unfolded tau protein identified from residual dipolar couplings and molecular simulation. J. Am. Chem. Soc., 129(16):5235-5243.

[Mustafi et al., 2006] Mustafi, R., Cerda, S., Chumsangsri, A., Fichera, A., and Bissonnette, M. (2006). Protein kinase-zeta inhibits collagen I-dependent and anchorage-independent growth and enhances apoptosis of human Caco-2 cells. Mol. Cancer Res., 4(9):683-694.

[Nasipuri, 2012] Nasipuri, D. (2012). Stereochemistry of Organic Compounds: Principles and Applications. New Academic Science, 4th revised ed. edition.

[Nelder and Mead, 1965] Nelder, J. and Mead, R. (1965). A simplex-method for function minimization. Comput. J., 7(4):308-313.

[Newman and Cragg, 2007] Newman, D. J. and Cragg, G. M. (2007). Natural products as sources of new drugs over the last 25 years. J. Nat. Prod., 70(3):461-477.

[Newman and Cragg, 2012] Newman, D. J. and Cragg, G. M. (2012). Natural products as sources of new drugs over the 30 years from 1981 to 2010. J. Nat. Prod., 75(3):311-335.

[Nicolaou and Snyder, 2005] Nicolaou, K. C. and Snyder, S. A. (2005). Chasing molecules that were never there: misassigned natural products and the role of chemical synthesis in modern structure elucidation. Angew. Chem. Int. Ed. Engl., 44(7):1012-1044.

[O'Boyle et al., 2011] O'Boyle, N. M., Banck, M., James, C. A., Morley, C., Vandermeersch, T., and Hutchison, G. R. (2011). Open Babel: An open chemical toolbox. J. Cheminformatics, 3(33).

[Omura et al., 1980] Omura, S., Matsubara, H., Nakagawa, A., Furusaki, A., and Matsumoto, T. (1980). X-ray crystallography of protylonolide and absolute configuration of tylosin. J. Antibiot. (Tokyo), 33(8):915-917. 
[Oostenbrink et al., 2004] Oostenbrink, C., Villa, A., Mark, A., and van Gunsteren, W. (2004). A biomolecular force field based on the free enthalpy of hydration and solvation: The GROMOS force-field parameter sets 53A5 and 53A6. J. Comput. Chem., 25(13):1656-1676.

[Palmer et al., 1991] Palmer, A., Cavanagh, J., Wright, P., and Rance, M. (1991). Sensitivity improvement in proton-detected 2-dimensional heteronuclear correlation NMR-spectroscopy. J. Magn. Reson., 93(1):151-170.

[Pérez-Balado et al., 2011] Pérez-Balado, C., Sun, H., Griesinger, C., de Lera, A. R., and Navarro-Vázquez, A. (2011). Residual dipolar coupling enhanced NMR spectroscopy and chiroptics: A powerful combination for the complete elucidation of symmetrical small molecules. Chemistry, 17(43):11983-11986.

[Petrovic et al., 2010] Petrovic, A. G., Navarro-Vazquez, A., and Lorenzo AlonsoGomez, J. (2010). From relative to absolute configuration of complex natural products: Interplay between NMR, ECD, VCD, and ORD assisted by ab initio calculations. Curr. Org. Chem., 14(15):1612-1628.

[Purcell et al., 1946] Purcell, E. M., Torrey, H. C., and Pound, R. V. (1946). Resonance absorption by nuclear magnetic moments in a solid. Phys. Rev., 69(1-2):3738.

[Ravelo et al., 2004] Ravelo, A., Estevez-Braun, A., Chavez-Orellana, H., PerezSacau, E., and Mesa-Siverio, D. (2004). Recent studies on natural products as anticancer agents. Curr. Top. Med. Chem., 4(2):241-265.

[Reinscheid et al., 2010] Reinscheid, U. M., Koeck, M., Cychon, C., Schmidts, V., Thiele, C. M., and Griesinger, C. (2010). The absolute configuration of dibromopalau'amine. Eur. J. Org. Chem., 2010(36):6900-6903.

[Riccio et al., 2003] Riccio, R., Bifulco, G., Cimino, P., C, B., and L, G.-P. (2003). Stereochemical analysis of natural products. Approaches relying on the combination of NMR spectroscopy and computational methods. Pure Appl. Chem., 75(2-3):295-308.

[Sajan et al., 2009] Sajan, M. P., Standaert, M. L., Nimal, S., Varanasi, U., Pastoor, T., Mastorides, S., Braun, U., Leitges, M., and Farese, R. V. (2009). The critical role of atypical protein kinase $\mathrm{C}$ in activating hepatic SREBP-1c and NF kappa B in obesity. J. Lipid Res., 50(6):1133-1145.

[Sánchez-Pedregal et al., 2009] Sánchez-Pedregal, V. M., Santamaría-Fernández, R., and Navarro-Vázquez, A. (2009). Residual dipolar couplings of freely rotating 
groups in small molecules. Stereochemical assignment and side-chain conformation of 8-phenylmenthol. Org. Lett., 11(7):1471-1474.

[Saupe, 1968] Saupe, A. (1968). Recent results in field of liquid crystals. Angew. Chem. Int. Edit., 7(2):97-112.

[Schmidt et al., 2009] Schmidt, M., Haberz, P., Leonov, A., and Griesinger, C. (2009). Future directions in NMR (INSA), chapter: Towards a complete set of alignment media for organic solvents: APS and PPH gel. Springer-Verlag Berlin Heidelberg, 1. edition.

[Schmidt et al., 2012] Schmidt, M., Sun, H., Rogne, P., Scriba, G. K., Griesinger, C., Kuhn, L. T., and Reinscheid, U. M. (2012). Determining the absolute configuration of $(+)$-mefloquine $\mathrm{HCl}$, the side-effect-reducing enantiomer of the antimalaria drug Lariam. J. Am. Chem. Soc., 134(6):3080-3083.

[Schmidt-Rohr and W, 1996] Schmidt-Rohr, K. and W, S. H. (1996). Multidimensional Solide-State NMR and Polymers. Academic Press, 2. edition.

[Schuetz et al., 2007] Schuetz, A., Junker, J., Leonov, A., Lange, O. F., Molinski, T. F., and Griesinger, C. (2007). Stereochemistry of sagittamide A from residual dipolar coupling enhanced NMR. J. Am. Chem. Soc., 129(49):15114-15115.

[Schuetz et al., 2008] Schuetz, A., Murakami, T., Takada, N., Junker, J., Hashimoto, M., and Griesinger, C. (2008). RDC-enhanced NMR spectroscopy in structure elucidation of sucro-neolambertellin. Angew. Chem. Int. Ed. Engl., 47(11):2032-2034.

[Scott et al., 1999] Scott, W., Hunenberger, P., Tironi, I., Mark, A., Billeter, S., Fennen, J., Torda, A., Huber, T., Kruger, P., and van Gunsteren, W. (1999). The GROMOS biomolecular simulation program package. J. Phys. Chem. A, 103(19):3596-3607.

[Seco et al., 2004] Seco, J. M., Quiñoá, E., and R, R. (2004). The assignment of absolute configuration by NMR. Chem. Rev., 104:17-117.

[Seiple et al., 2011] Seiple, I. B., Su, S., Young, I. S., Nakamura, A., Yamaguchi, J., Jorgensen, L., Rodriguez, R. A., O’Malley, D. P., Gaich, T., Köck, M., and Baran, P. S. (2011). Enantioselective total syntheses of (-)-palau'amine, (-)-axinellamines, and (-)-massadines. J. Am. Chem. Soc., 133(37):14710-14726.

[Shen et al., 2009] Shen, T., Wang, X.-N., and Lou, H.-X. (2009). Natural stilbenes: an overview. Nat. Prod. Rep., 26(7):916-935. 
[Stevensson et al., 2003] Stevensson, B., Sandstrom, D., and Maliniak, A. (2003). Conformational distribution functions extracted from residual dipolar couplings: A hybrid model based on maximum entropy and molecular field theory. J. Chem. Phys., 119(5):2738-2746.

[Sun et al., 2011a] Sun, H., d'Auvergne, E. J., Reinscheid, U. M., Dias, L. C., Andrade, C. K., Rocha, R. O., and Griesinger, C. (2011a). Bijvoet in solution reveals unexpected stereoselectivity in a Michael addition. Chemistry, 17(6):1811-1817.

[Sun et al., 2010] Sun, H., Dinan, L., Lafont, R., Suksamrarn, A., Griesinger, C., Reinscheid, U., and Lapenna, S. (2010). Absolute configuration and docking study of canescensterone, a potent phytoecdysteroid, with non-lepidopteran ecdysteroid receptor selectivity. Eur. J. Org. Chem., 2010(30):5791-5799.

[Sun et al., 2011b] Sun, H., Reinscheid, U. M., Whitson, E. L., d'Auvergne, E. J., Ireland, C. M., Navarro-Vázquez, A., and Griesinger, C. (2011b). Challenge of large-scale motion for residual dipolar coupling based analysis of configuration: The case of fibrosterol sulfate A. J. Am. Chem. Soc., 133(37):14629-14636.

[Sun et al., 2005] Sun, R., Gao, P., Chen, L., Ma, D., Wang, J., Oppenheim, J., and Zhang, N. (2005). Protein kinase C zeta is required for epidermal growth factorinduced chemotaxis of human breast cancer cells. Cancer Res., 65(4):1433-1441.

[Thaning et al., 2008] Thaning, J., Stevensson, B., Ostervall, J., Naidoo, K. J., Widmalm, G., and Maliniak, A. (2008). NMR studies of molecular conformations in alpha-cyclodextrin. J. Phys. Chem. B, 112(29):8434-8436.

[Thiele, 2008] Thiele, C. M. (2008). Residual dipolar couplings (RDCs) in organic structure determination. Eur. J. Org. Chem., (34):5673-5685.

[Thiele and Berger, 2003] Thiele, C. M. and Berger, S. (2003). Probing the diastereotopicity of methylene protons in strychnine using residual dipolar couplings. Org. Lett., 5(5):705-708.

[Thiele et al., 2011] Thiele, C. M., Pomerantz, W. C., Abbott, N. L., and Gellman, S. H. (2011). Lyotropic liquid crystalline phases from helical beta-peptides as alignment media. Chem. Commun. (Camb.), 47(1):502-504.

[Thiele et al., 2009] Thiele, C. M., Schmidts, V., Böttcher, B., Louzao, I., Berger, R., Maliniak, A., and Stevensson, B. (2009). On the treatment of conformational flexibility when using residual dipolar couplings for structure determination. Angew. Chem. Int. Ed. Engl., 48(36):6708-6712. 
[Thrippleton and Keeler, 2003] Thrippleton, M. J. and Keeler, J. (2003). Elimination of zero-quantum interference in two-dimensional NMR spectra. Angew. Chem. Int. Ed. Engl., 42(33):3938-3941.

[Tjandra and Bax, 1997] Tjandra, N. and Bax, A. (1997). Direct measurement of distances and angles in biomolecules by NMR in a dilute liquid crystalline medium. Science, 278(5340):1111-1114.

[Trigo-Mouriño et al., 2011] Trigo-Mouriño, P., Navarro-Vázquez, A., Ying, J., Gil, R. R., and Bax, A. (2011). Structural discrimination in small molecules by accurate measurement of long-range proton-carbon NMR residual dipolar couplings. Angew. Chem. Int. Ed. Engl., 50(33):7576-7580.

[Trigo-Mouriño et al., 2010] Trigo-Mouriño, P., Santamaría-Fernández, R., Sánchez-Pedregal, V. M., and Navarro-Vázquez, A. (2010). Conformational analysis of an isoquinolinium hydrochloride in water using residual dipolar couplings. J. Org. Chem., 75(9):3101-3104.

[Tsukamoto et al., 2010] Tsukamoto, T., Nakata, R., Tamura, E., Kosuge, Y., Kariya, A., Katsukawa, M., Mishima, S., Ito, T., Iinuma, M., Akao, Y., Nozawa, Y., Arai, Y., Namura, S., and Inoue, H. (2010). Vaticanol C, a resveratrol tetramer, activates PPAR alpha and PPAR beta/delta in vitro and in vivo. Nutr. Metab., 7 .

[Verdier et al., 2003] Verdier, L., Sakhaii, P., Zweckstetter, M., and Griesinger, C. (2003). Measurement of long range H,C couplings in natural products in orienting media: a tool for structure elucidation of natural products. J. Magn. Reson., 163(2):353-359.

[Whitson et al., 2009] Whitson, E. L., Bugni, T. S., Chockalingam, P. S., Concepcion, G. P., Feng, X., Jin, G., Harper, M. K., Mangalindan, G. C., McDonald, L. A., and Ireland, C. M. (2009). Fibrosterol sulfates from the Philippine Sponge Lissodendoryx (Acanthodoryx) fibrosa: Sterol dimers that inhibit PKC zeta. J. Org. Chem., 74(16):5902-5908.

[Whitson et al., 2012] Whitson, E. L., Sun, H., Thomas, C. L., Henrich, C. J., Sayers, T. J., McMahon, J. B., Griesinger, C., and McKee, T. C. (2012). Synergistic TRAIL sensitizers from Barleria alluaudii and Diospyros maritima. J. Nat. Prod., 75(3):394-399.

[Wider et al., 1984] Wider, G., Macura, S., Kumar, A., Ernst, R., and Wuthrich, K. (1984). Homonuclear two-dimensional H-1-NMR of proteins - experimental procedures. J. Magn. Reson., 56(2):207-234. 
[Williamson et al., 2000] Williamson, R. T., Márquez, B. L., Gerwick, W. H., and Kövér, K. E. (2000). One- and two-dimensional gradient-selected HSQMBC NMR experiments for the efficient analysis of long-range heteronuclear coupling constants. Magn. Reson. Chem., 38(4):265-273.

[Wodrich et al., 2007] Wodrich, M. D., Corminboeuf, C., Schreiner, P. R., Fokin, A. A., and von Ragué Schleyer, P. (2007). How accurate are DFT treatments of organic energies? Org. Lett., 9(10):1851-1854.

[Wollrab, 2009] Wollrab, A. (2009). Organische Chemie: Eine Einführung für Lehramts- und Nebenfachstudenten. Springer-Verlag Berlin Heidelberg, 3. edition.

[Yan et al., 2004] Yan, J., Delaglio, F., Kaerner, A., Kline, A. D., Mo, H., Shapiro, M. J., Smitka, T. A., Stephenson, G. A., and Zartler, E. R. (2004). Complete relative stereochemistry of multiple stereocenters using only residual dipolar couplings. J. Am. Chem. Soc., 126(15):5008-5017.

[Yan et al., 2003] Yan, J., Kline, A. D., Mo, H., Shapiro, M. J., and Zartler, E. R. (2003). A novel method for the determination of stereochemistry in six-membered chairlike rings using residual dipolar couplings. J. Org. Chem., 68(5):1786-1795.

[Yura et al., 1987] Yura, T., Iwasawa, N., and Mukaiyama, T. (1987). New possibilities in the Michael reaction by the use of tin(II) enolates. Chem. Lett., (5):791-794.

[Yura et al., 1988] Yura, T., Iwasawa, N., and Mukaiyama, T. (1988). The enantioselective Michael reaction of Tin(II) enolates. Chem. Lett., (6):1021-1024.

[Zhao et al., 2007] Zhao, C., Cai, M., Zhang, Y., Liu, Y., Sun, R., and Zhang, N. (2007). Development of a microscopy-based assay for protein kinase C zeta activation in human breast cancer cells. Anal. Biochem., 362(1):8-15.

[Zweckstetter, 2008] Zweckstetter, M. (2008). NMR: prediction of molecular alignment from structure using the PALES software. Nat. Protoc., 3(4):679-690.

[Zweckstetter and Bax, 2000] Zweckstetter, M. and Bax, A. (2000). Prediction of sterically induced alignment in a dilute liquid crystalline phase: Aid to protein structure determination by NMR. J. Am. Chem. Soc., 122(15):3791-3792. 


\section{Appendix A}

\section{Additional data}

\section{A.1 Additional data for LLG1}

Table A.1: Important dihedral angles (deg) of possible conformers 1-12 for both possible relative configurations of LLG1. All structures were optimized with DFT at the B3LYP/6$31 \mathrm{G}(\mathrm{d})$ level using the IEFPCM solvent continuum model with DMSO as the solvent.

\begin{tabular}{llrrr}
\hline Configuration & Conformer & $\begin{array}{c}\mathrm{H} 21-\mathrm{C} 21 \\
\text {-N-C23 }\end{array}$ & $\begin{array}{c}\mathrm{H} 12-\mathrm{C} 12 \\
\text {-C21-H21 }\end{array}$ & $\begin{array}{c}\text { O-C12 } \\
\text {-C11-C10 }\end{array}$ \\
\hline$(12 S, 21 S)$ or & 1 & -173.5 & 60.9 & -65.9 \\
$(12 R, 21 R)$ & 2 & 11.7 & 63.1 & -64.5 \\
& 3 & -150.7 & -58.5 & -66.9 \\
& 4 & 18.6 & -66.2 & -65.9 \\
& 5 & -175.6 & -173.3 & -58.6 \\
& 6 & 0.5 & -169.8 & -59.5 \\
& 7 & -177.4 & -170.9 & -145.7 \\
& 8 & 0.4 & -170.0 & -146.8 \\
& 9 & -141.5 & -59.5 & -161.3 \\
& 10 & 17.5 & -58.5 & -146.5 \\
& 11 & -177.6 & 60.3 & -171.2 \\
& 12 & 12.9 & 67.6 & -168.4 \\
\hline$(12 S, 21 R)$ or & 1 & -17.0 & -62.1 & -64.1 \\
& 2 & 156.6 & -63.8 & -64.8 \\
& 3 & -1.7 & -173.2 & -62.7 \\
& 3 & 174.8 & -178.7 & -61.5 \\
& 4 & -17.5 & 67.9 & -66.8 \\
& 5 & 151.2 & 63.5 & -66.9 \\
& 6 & -16.6 & 67.2 & -177.1 \\
\hline
\end{tabular}


Table A.1: Important dihedral angles (deg) of possible conformers 1-12 for both possible relative configurations of LLG1. All structures were optimized with DFT at the B3LYP/6$31 \mathrm{G}(\mathrm{d})$ level using the IEFPCM solvent continuum model with DMSO as the solvent.

\begin{tabular}{llrcl}
\hline Configuration & Conformer & $\begin{array}{c}\mathrm{H} 21-\mathrm{C} 21 \\
\text {-N-C23 }\end{array}$ & $\begin{array}{c}\mathrm{H} 12-\mathrm{C} 12 \\
-\mathrm{C} 21-\mathrm{H} 21\end{array}$ & $\begin{array}{c}\text { O-C12 } \\
\text {-C11-C10 }\end{array}$ \\
\hline 8 & 154.5 & 61.9 & -179.5 \\
9 & 1.3 & -177.0 & -161.2 \\
10 & 171.6 & -177.3 & -152.2 \\
11 & -16.5 & -60.7 & -155.0 \\
12 & 164.6 & -62.5 & -162.0 \\
\hline
\end{tabular}


Table A.2: Relative energy and relative Gibbs free energy of the 12 possible conformers of LLG1 with the configuration $(12 S, 21 S)$ or $(12 R, 21 R)$. The DFT calculations were performed at the B3LYP/6-31G(d) level using the IEFPCM solvent continuum model with DMSO as the solvent. Energy and Gibbs free energy were calculated using the equations in Appendix B, and all computations were performed at $298 \mathrm{~K}$ and $1 \mathrm{~atm}$.

\begin{tabular}{lll}
\hline Conformer & $\Delta E\left(\mathrm{kcal} \cdot \mathrm{mol}^{-1}\right)$ & $\Delta G\left(\mathrm{kcal} \cdot \mathrm{mol}^{-1}\right)$ \\
\hline 1 & 3.8 & 4.2 \\
2 & 0.0 & 0.0 \\
3 & 1.8 & 2.6 \\
4 & 4.9 & 5.0 \\
5 & 0.9 & 0.4 \\
6 & 1.4 & 2.3 \\
7 & 2.9 & 3.4 \\
8 & 3.4 & 4.6 \\
9 & 3.7 & 4.1 \\
10 & 7.8 & 8.8 \\
11 & 0.4 & 1.1 \\
12 & 4.9 & 5.5 \\
\hline
\end{tabular}

Table A.3: Experimental and computed ORD values of LLG1 at different wavelengths. The computed ORD values are calculated from the RDC-derived conformer 11 for the configuration $(12 R, 21 R)$ and $(12 S, 21 S)$. ORD computations were performed at the B3LYP $/ 6$ $31 \mathrm{G}(\mathrm{d})$ level using the IEFPCM solvent continuum model with DMSO as the solvent. The measurements were performed at $20{ }^{\circ} \mathrm{C}$.

\begin{tabular}{lccc}
\hline$\lambda(\mathrm{nm})$ & {$[\alpha]^{\exp }(\mathrm{deg})$} & $\begin{array}{c}{[\alpha]^{\text {DFT }}(\mathrm{deg})} \\
(12 R, 21 R)\end{array}$ & $(12 S, 21 S)$ \\
\hline $589(\mathrm{Na})$ & -58.22 & -60.08 & 60.08 \\
$578(\mathrm{Hg})$ & -64.69 & -63.23 & 63.23 \\
$546(\mathrm{Hg})$ & -71.16 & -72.23 & 72.23 \\
$436(\mathrm{Hg})$ & -181.13 & -136.29 & 136.29 \\
$365(\mathrm{Hg})$ & -491.63 & -382.21 & 383.21 \\
\hline
\end{tabular}



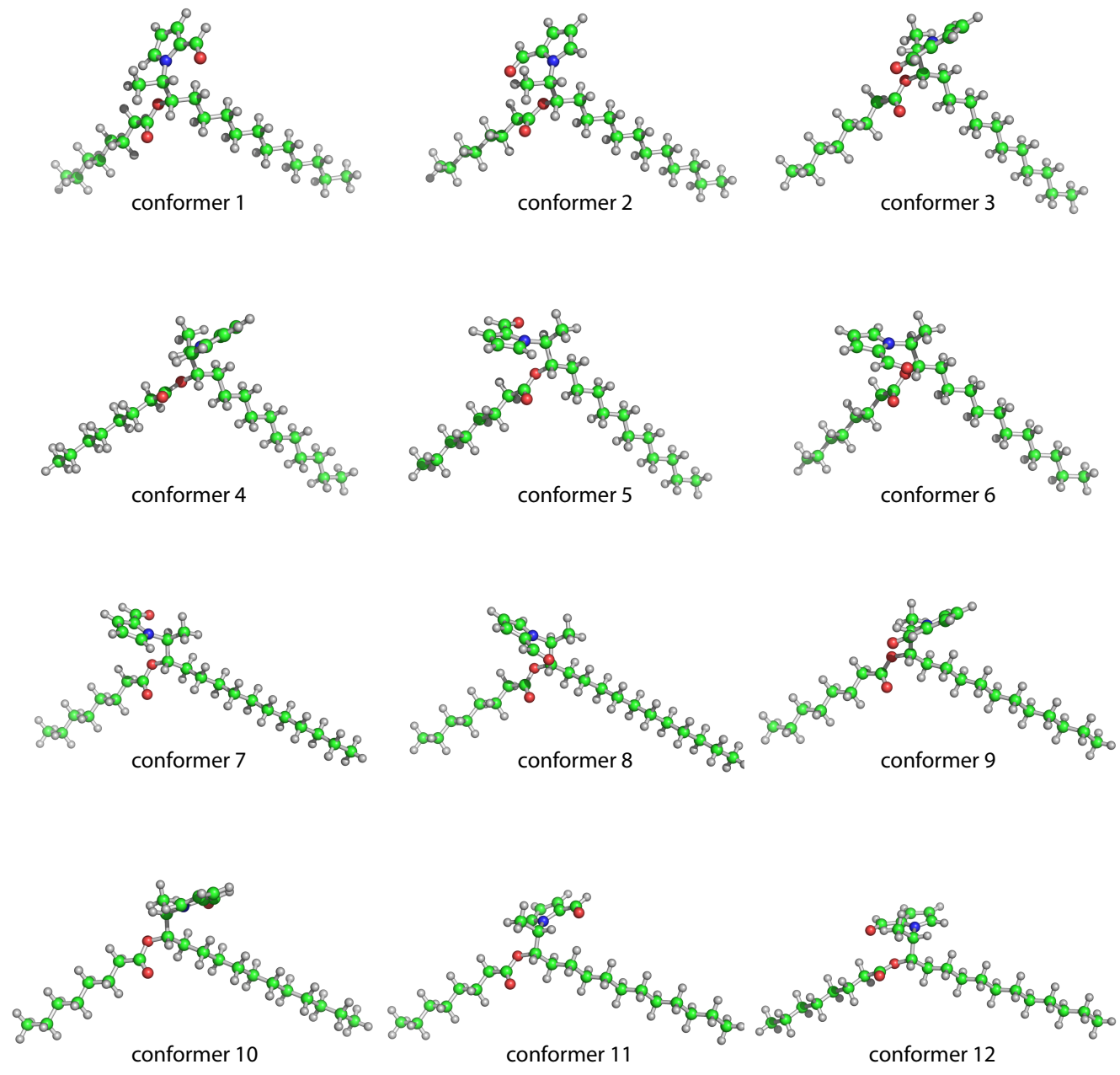

Figure A.1: 12 possible conformers of LLG1 with the configuration of $(12 R, 21 R)$. All structures were optimized at the B3LYP/6-31G(d) level using the IEFPCM solvent continuum model with DMSO as the solvent. 

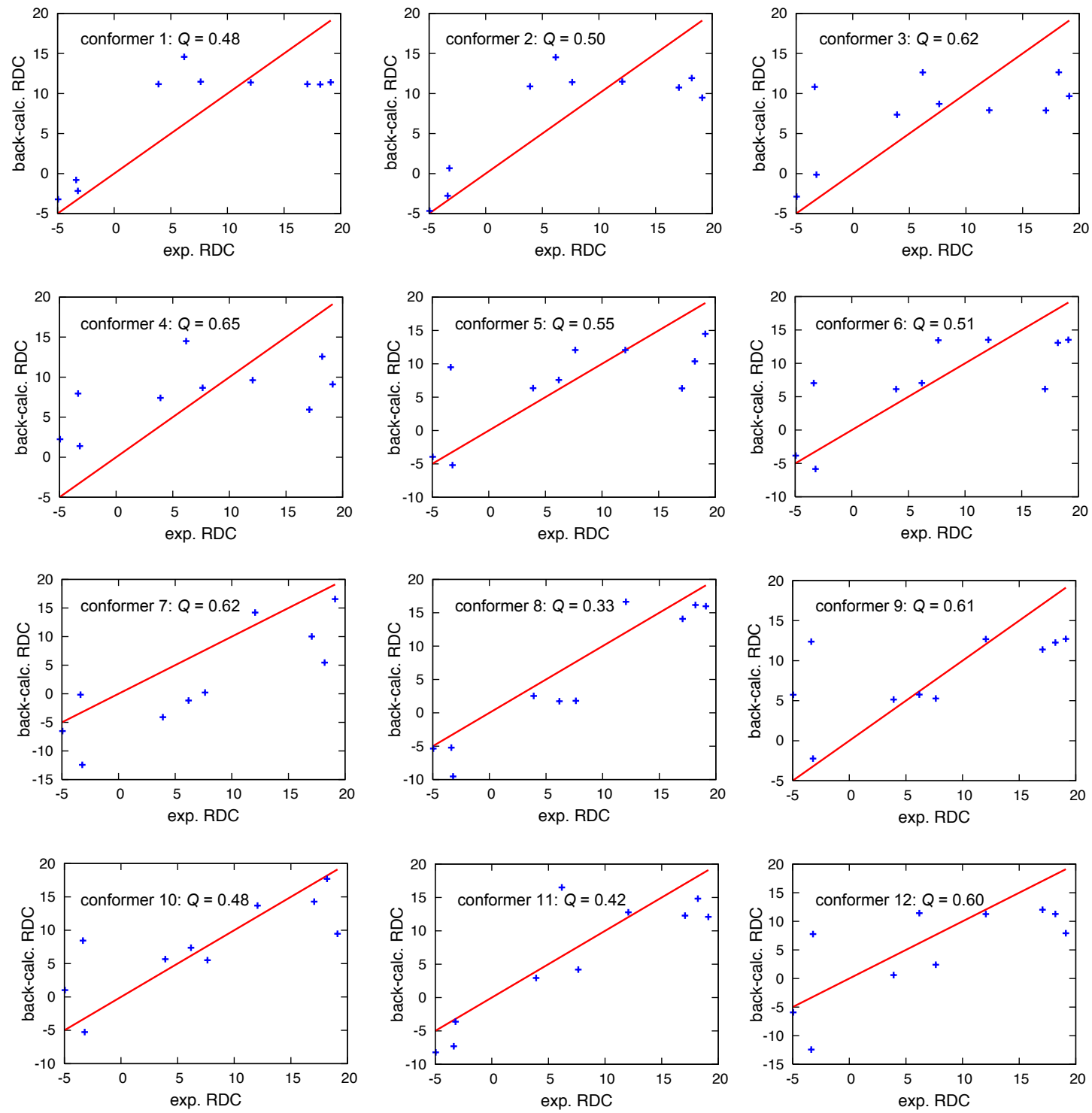

Figure A.2: Comparison of the correlations of experimental and back-calculated RDCs of 12 possible conformers of LLG1 with the configuration $(12 S, 21 R)$ or $(12 R, 21 S)$. 

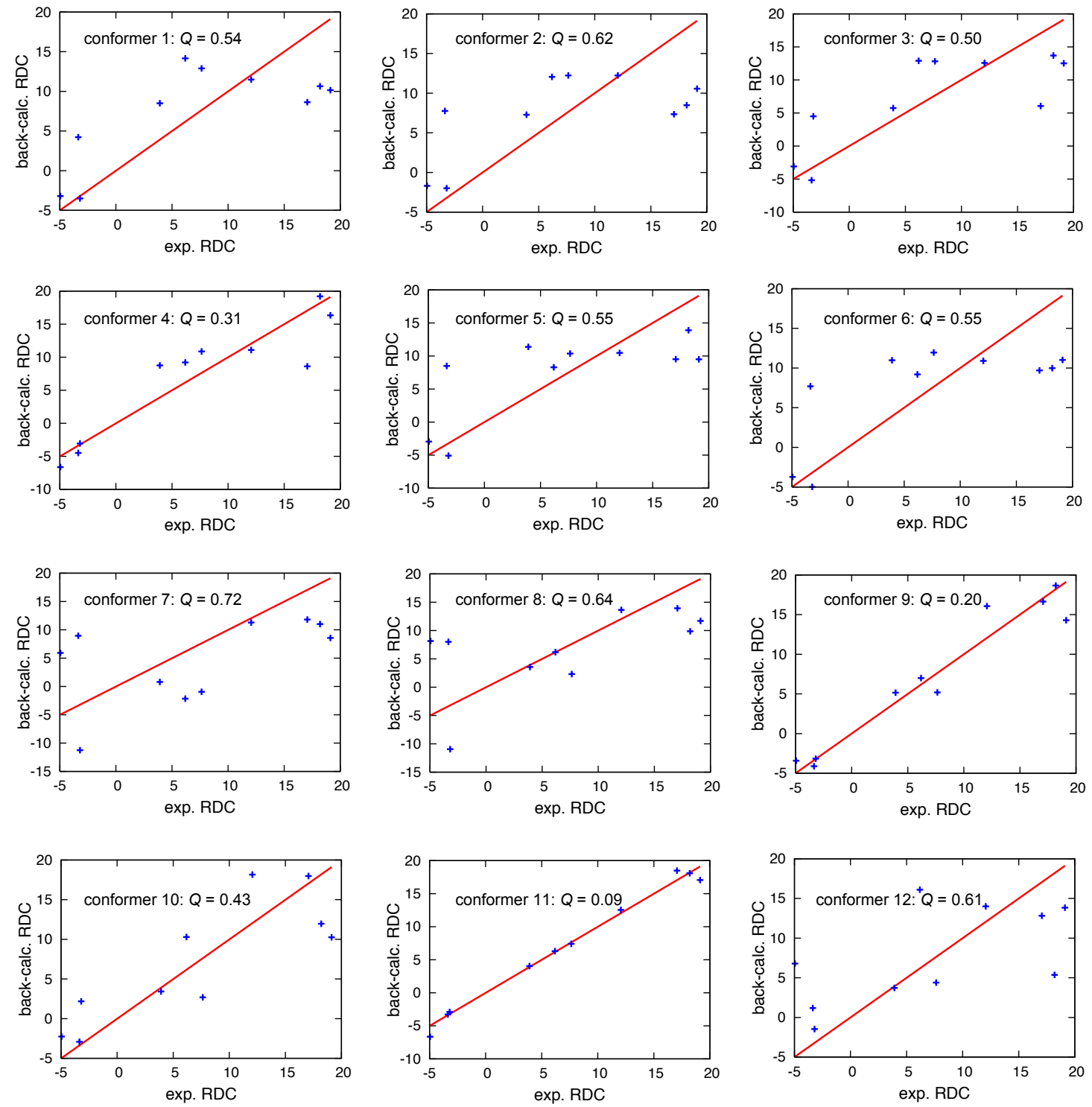

Figure A.3: Comparison of the correlations of experimental and back-calculated RDCs of 12 possible conformers of LLG1 with the configuration $(12 R, 21 R)$ or $(12 S, 21 S)$. 


\section{A.2 Additional data for vatiparol}

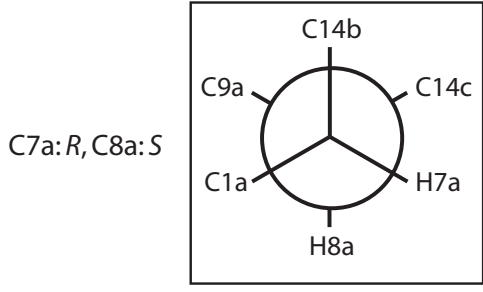

a

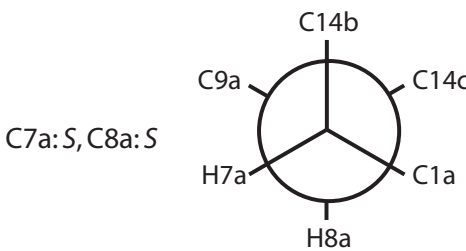

d

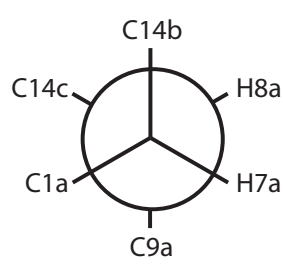

b

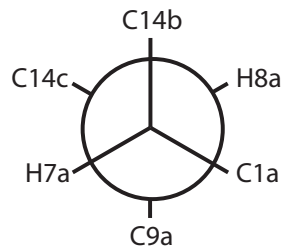

e

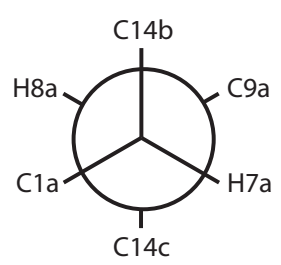

C

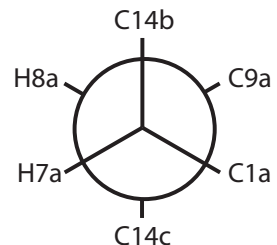

f

\begin{tabular}{lll}
\hline${ }^{3} J(\mathrm{H} 7 \mathrm{a}-\mathrm{H} 8 \mathrm{a})$ & $2.8 \mathrm{~Hz}$ & extracted from $1 \mathrm{D}-{ }^{1} \mathrm{H}$ \\
${ }^{3} \mathrm{~J}(\mathrm{H} 7 \mathrm{a}-\mathrm{C} 9 \mathrm{a})$ & $8.2 \mathrm{~Hz}$ & extracted from $\left[{ }^{1} \mathrm{H}-{ }^{13} \mathrm{C}\right]-\mathrm{HSQMBC}$ \\
${ }^{3} \mathrm{~J}(\mathrm{H} 7 \mathrm{a}-\mathrm{C} 14 \mathrm{c})$ & $3.0 \mathrm{~Hz}$ & extracted from $\left[{ }^{1} \mathrm{H}-{ }^{13} \mathrm{C}\right]-\mathrm{HSQMBC}$ \\
${ }^{3} \mathrm{~J}(\mathrm{H} 8 \mathrm{a}-\mathrm{C} 1 \mathrm{a})$ & $2.8 \mathrm{~Hz}$ & extracted from $\left[{ }^{1} \mathrm{H}-{ }^{13} \mathrm{C}\right]-\mathrm{HSQMBC}$ \\
${ }^{3} \mathrm{~J}(\mathrm{H} 8 \mathrm{a}-\mathrm{C} 14 \mathrm{~b})$ & $8.0 \mathrm{~Hz}$ & extracted from $\left[{ }^{1} \mathrm{H}-{ }^{13} \mathrm{C}\right]-\mathrm{HSQMBC}$ \\
\hline
\end{tabular}

Figure A.4: Newman projections of 6 possible conformers $(\mathbf{a}-\mathbf{f})$ for the dihedral C7aC8a of vatiparol. Three conformers on top have a configuration of $(7 \mathrm{a} R, 8 \mathrm{a} S)$, whereas the three conformers at the bottom have a configuration of $(7 \mathrm{a} S, 8 \mathrm{a} S)$. A small ${ }^{3} J(\mathrm{H} 7 \mathrm{a}-$ $\mathrm{H} 7 \mathrm{~b})$ coupling excludes the conformers $\mathbf{c}$ and $\mathbf{e}$ as the main conformation. Large ${ }^{3} J(\mathrm{H} 7 \mathrm{a}-$ C9a) and ${ }^{3} J$ (H8a-C14b) couplings, together with small ${ }^{3} J(\mathrm{H} 7 \mathrm{a}-\mathrm{C} 14 \mathrm{c})$ and ${ }^{3} J(\mathrm{H} 8 \mathrm{a}-\mathrm{C} 1 \mathrm{a})$ couplings determine conformer $\mathbf{a}$ as the main conformation. The relative configuration of $\mathrm{C} 7 \mathrm{a}$ and $\mathrm{C} 8 \mathrm{a}$ was established as $(7 \mathrm{a} R, 8 \mathrm{a} S)$ or $(7 \mathrm{a} S, 8 \mathrm{a} R)$. 
Table A.4: NOE violation $U$ of configuration SRSSRSSR (or its enantiomer $R S R R S R R S$ ), calculated from Equation 3.8. An uncertainty of $0.4 \AA$ was assumed for each proton pair. The structure model for the NOE analysis was performed at the B3LYP/6$31 \mathrm{G}(\mathrm{d})$ level using the IEFPCM solvent continuum model with DMSO as the solvent

\begin{tabular}{llll}
\hline Atoms & $U\left(\AA^{2}\right)$ & Atoms & $U\left(\AA^{2}\right)$ \\
\hline H2a,6a-H7a & 0.0 & H3b,5b-H7b & 0.2 \\
H2a,6a-H8a & 0.0 & H3b,5b-H10b & 0.0 \\
H2a,6a-H10a & 0.0 & H7b-H8b & 0.0 \\
H2a,6a-H2,6b & 0.0 & H7b-H10b & 0.0 \\
H2a,6a-H8b & 0.0 & H7b-H2c,6c & 0.0 \\
H2a,6a-H10b & 0.0 & H7b-H3c,5c & 0.0 \\
H3a,5a-H7a & 0.0 & H7b-H8c & 0.0 \\
H3a,5a-H8a & 0.1 & H8b-H10b & 0.0 \\
H3a,5a-H8b & 0.0 & H8b-H8c & 0.0 \\
H3a,5a-H12c & 0.0 & H10b-H12b & 0.0 \\
H7a-H8a & 0.0 & H10b-H2c,6c & 0.0 \\
H7a-H10a & 0.0 & H10b-H3c,5c & 0.0 \\
H7a-H8b & 0.0 & H10b-H8c & 0.0 \\
H7a-H12b & 0.1 & H12b-H2c,6c & 0.0 \\
H7a-H7c & 0.0 & H12b-H3c,5c & 0.0 \\
H7a-H8c & 0.0 & H12b-H7c & 0.0 \\
H7a-H12a & 0.0 & H12b-H8c & 0.0 \\
H8a-H10a & 0.0 & H12b-H10c & 0.2 \\
H8a-H8b & 0.0 & H2c,6c-H7c & 0.0 \\
H8a-H8c & 0.0 & H2c,6c-H8c & 0.0 \\
H8a-H12c & 0.0 & H2c,6c-H10c & 0.0 \\
H10a-H12a & 0.0 & H3c,5c-H7c & 0.1 \\
H10a-H8c & 0.0 & H3c,5c-H8c & 0.1 \\
H10a-H12c & 0.0 & H3c,5c-H10c & 0.0 \\
H12a-H7b & 0.0 & H7c-H8c & 0.0 \\
H2b,6b-H7b & 0.0 & H7c-H10c & 0.0 \\
H2b,6b-H8b & 0.0 & H8c-H10c & 0.0 \\
H2b,6b-H10b & 0.0 & H10c-H12c & 0.0 \\
\hline & & &
\end{tabular}



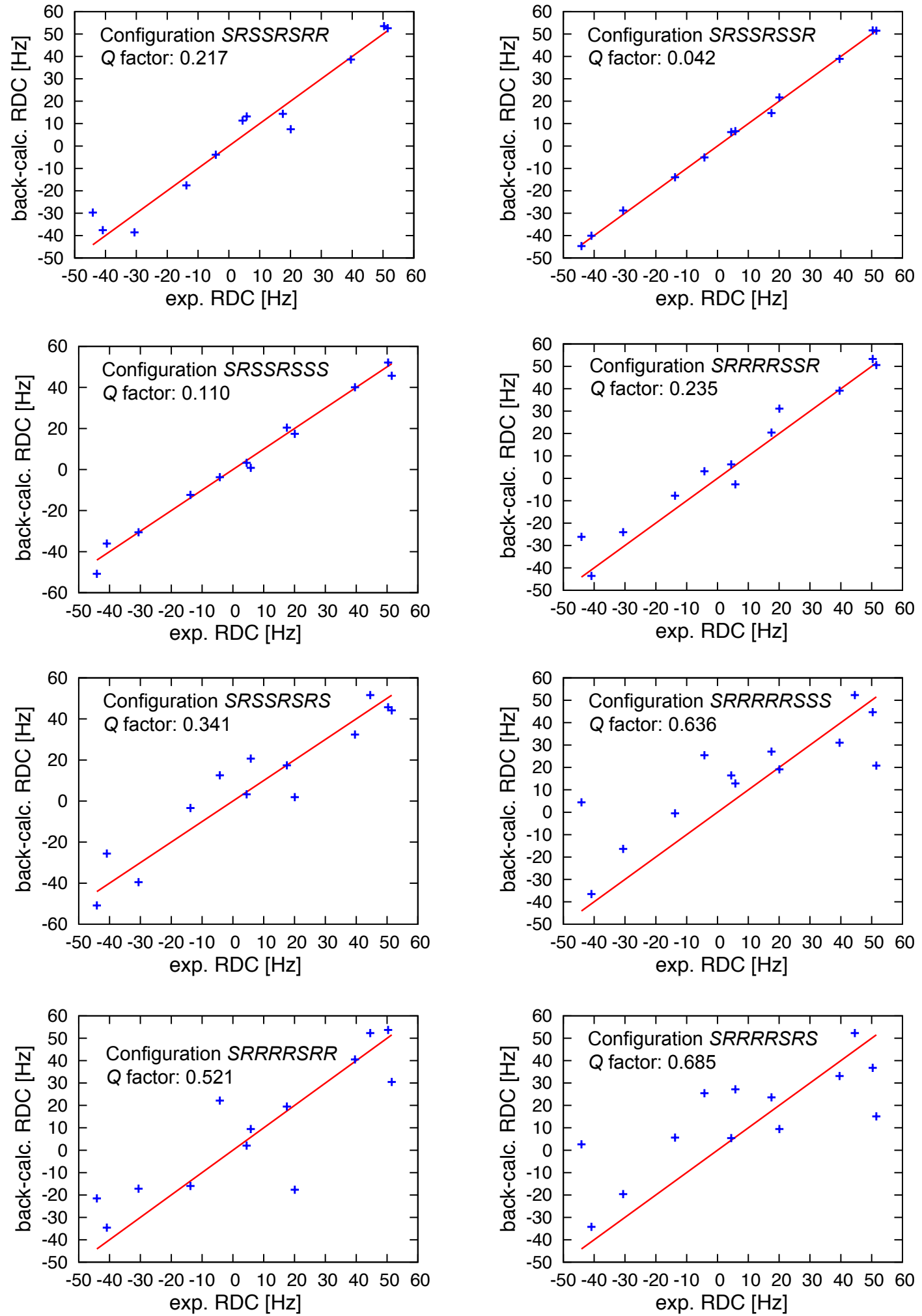

Figure A.5: Correlation between experimental and back-calculated RDCs for 8 possible diastereomers of vatiparol. 
Table A.5: Experimental and computed ORD values of vatiparol at different wavelengths. The computed ORD values are calculated from the RDC-derived conformations for the configuration SRSSRSRR. ORD computations were performed at the B3LYP/6-31G(d) level using the IEFPCM solvent continuum model with DMSO as the solvent. The ORD measurements were performed at $20{ }^{\circ} \mathrm{C}$.

\begin{tabular}{llllll}
\hline$\lambda(\mathrm{nm})$ & {$[\alpha]^{\exp }$} & \multicolumn{3}{c}{$[\alpha]^{\text {DFT }}$} & (deg) (Configuration: SRSSRSRR) \\
$($ Source) & $(\mathrm{deg})$ & Conformer 1 & Conformer 2 & Conformer 3 & Conformer 4 \\
\hline $589(\mathrm{Na})$ & 248.04 & 392.78 & 350.46 & 255.44 & 235.56 \\
$578(\mathrm{Hg})$ & 260.60 & 410.26 & 365.93 & 268.61 & 247.68 \\
$546(\mathrm{Hg})$ & 302.98 & 460.62 & 410.31 & 308.13 & 284.06 \\
$436(\mathrm{Hg})$ & 631.08 & 683.00 & 593.46 & 570.17 & 526.30 \\
\hline
\end{tabular}

To rationalize the biochemical construction of the novel carbon skeleton for vatiparol, the reactivity of the tautomeric resveratrol radicals A-F (Figure A.6) was examined computationally. The coupling between radicals $\mathrm{B}$ and $\mathrm{F}$ is highly probable since the most pronounced enthalpic $\left(\Delta H=-31.46 \mathrm{kcal} \cdot \mathrm{mol}^{-1}\right)$ and Gibbs free energetic changes $\left(\Delta G=-8.05 \mathrm{kcal} \cdot \mathrm{mol}^{-1}\right)$ can be expected from the $\mathrm{C}$ - $\mathrm{C}$ bond formation. This is generally in agreement with the superior electron delocalizability of radicals B and F. Furthermore, the presence of the 2,5,6,6-tetrasubstituted cyclohex4-ene-1,3-dione moiety in vatiparol implies that the 3,5-dihydroxyphenyl residue of a resveratrol unit has been repeatedly attacked during the in planta oligomerization process. As illustrated in Figure A.6, the biosynthesis of vatiparol could be driven by the $\mathrm{C}-\mathrm{C}$ bond formations through the radical-radical couplings and intramolecular nucleophilic cycloadditions. Interestingly, the configuration we determined for vatiparol is naturally reasonable, that is, energetically preferable in view of the steric hindrance, which tends to orient each pair of neighboring phenyl rings in a trans position. It is noteworthy that this hindrance-imprinted rationalization helps understand the stereochemical preference of the reported resveratrol oligomers, such as e-viniferin, vitisinols $\mathrm{B}$ and $\mathrm{D}$, and suffruticosol C. 

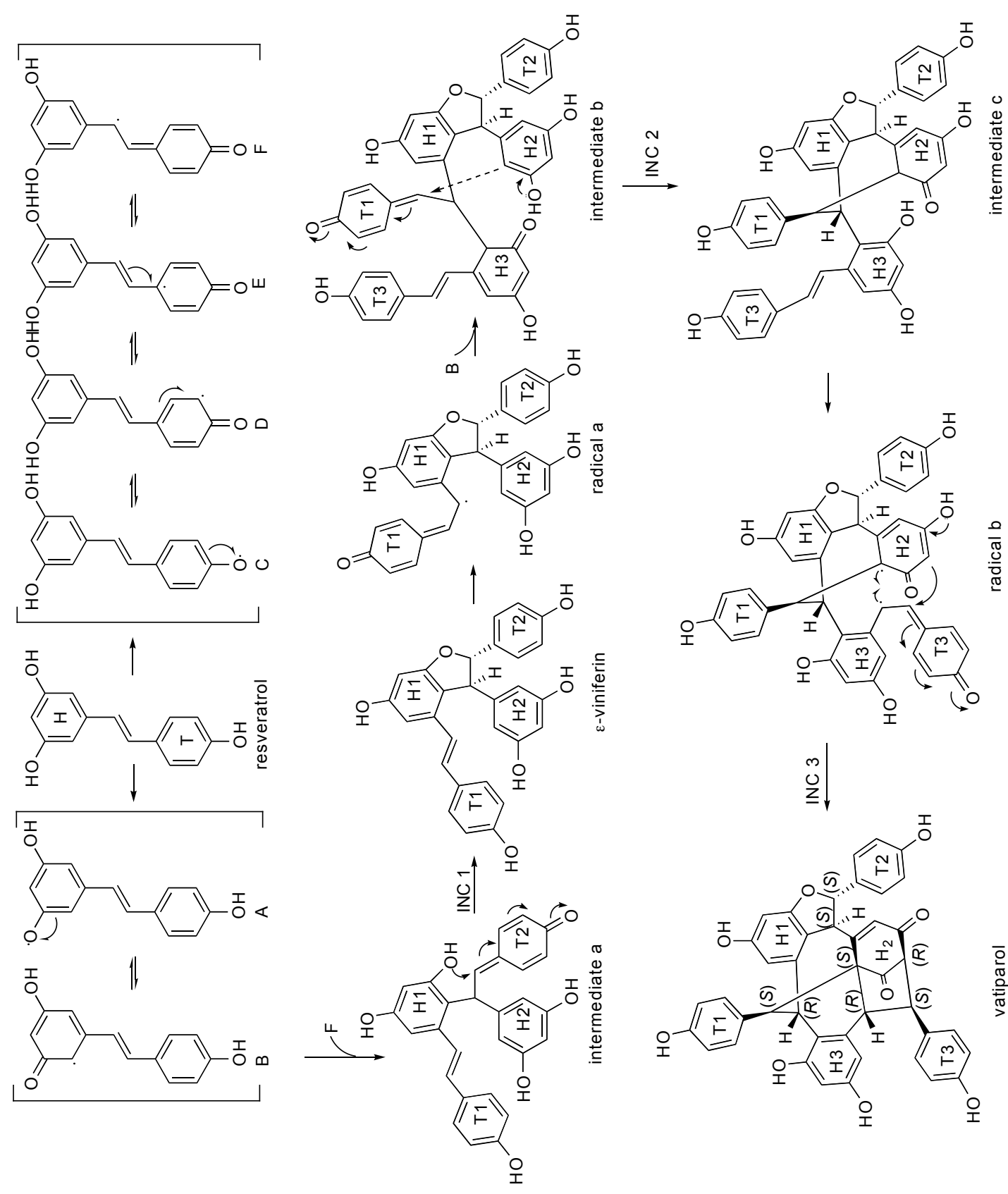

Figure A.6: Proposed biosynthetic pathway of vatiparol. H1-H3 and T1-T3 symbolize, respectively, 1,3-dihydroxy (Head) and hydroxy (Tail) benzene rings of the $1^{\text {st }}$ through $3^{\text {rd }}$ resveratrol units. A $-\mathrm{F}$ represent resveratrol radical tautomers with the $\mathrm{B}-\mathrm{F}$ coupling resulting in the most substantial enthalpic and Gibbs free energetic decreases. The novel carbon skeleton of vatiparol might have been primarily generated by a combination of the B-F coupling (-like) and intramolecular nucleophilic cycloaddition (INC). Concerning the configuration of vatiparol, the enzymatic catalysis and steric hindrance between $\mathrm{H} 2$ and $\mathrm{T} 2$ rings during INC 1 would accommodate collectively the configuration of the 2,3-dihydrobenzofuran moiety, and subsequently the repulsion of $\mathrm{H} 3$ against $\mathrm{T} 1$ and $\mathrm{T} 3$, encountered successively upon INC 2 and 3, could permit those of other chiral centers. 


\section{A.3 Additional data for naphth 1 and naphth 2}

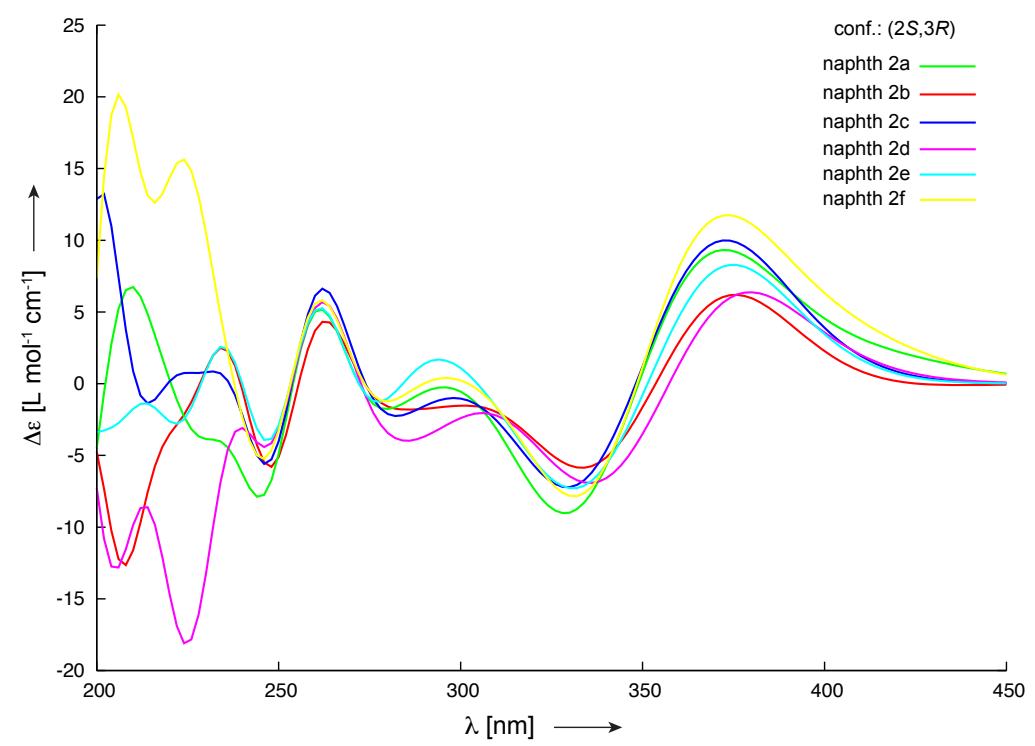

Figure A.7: Comparison of the computed ECD spectra for the six lowest energy conformers with a $(2 S, 3 R)$ configuration of naphth 2 . The calculations were performed with DFT at the B3LYP/6-31G(d) level using the IEFPCM solvent continuum model with methanol as the solvent. 
Table A.6: Carbon and proton chemical shifts of naphth 2 in $\mathrm{CD}_{3} \mathrm{OD}-d_{4}$ at $298 \mathrm{~K}$. The data were acquired by Dr. Emily L. Whitson.

\begin{tabular}{lcll}
\hline Carbon & Chemical shift $(\mathrm{ppm})$ & Proton & Chemical shift $(\mathrm{ppm})$ \\
\hline C1 & 198.8 & & \\
C2 & 64.7 & & \\
C3 & 60.4 & H3 & 3.84 \\
C4 & 192.4 & & \\
C4a & 133.9 & & \\
C5 & 119.9 & H5 & 7.46 \\
C6 & 138.1 & H6 & 7.66 \\
C7 & 125.3 & H7 & 7.26 \\
C8 & 163.0 & & \\
C8a & 116.0 & & \\
C1a' & 26.8 & H1a' & 2.98 \\
& & H1b' & 2.72 \\
C2 & 116.9 & H2' & 5.13 \\
C3 & 138.2 & & \\
C4' & 18.3 & H4' & 1.70 \\
C5' & 26.2 & H5' & 1.74 \\
\hline
\end{tabular}

Table A.7: Relevant long-range proton-carbon coupling constants of naphth 2 for the conformational analysis of the side chain. The measurements were performed using a $J$ resolved $\mathrm{HMBC}$ optimized for a $J_{\mathrm{CH}}$ coupling of $1 \mathrm{~Hz}$ and a scaling factor of 32 . The data were acquired by Dr. Emily L. Whitson.

\begin{tabular}{lclc}
\hline Atoms & Coupling constant $(\mathrm{Hz})$ & Atoms & Coupling constant $(\mathrm{Hz})$ \\
\hline H1a'-C1 & $\leq 2.0$ & H1b'-C1 & 2.7 \\
H1a'-C2 & -5.4 & H1b'-C2 & -7.7 \\
H1a'-C3 & 2.9 & H1b'-C3 & 3.6 \\
H2'-C2 & 2.1 & & \\
\hline
\end{tabular}


Table A.8: Important dihedral angles (deg) of optimized predominant conformers naphth 2a-2f with DFT at the B3LYP/6-31G(d) level using the IEFPCM solvent continuum model with methanol as the solvent.

\begin{tabular}{lrrrrrr}
\hline Dihedral & \multicolumn{5}{c}{ Dihedral angle (deg) } \\
& $\mathbf{2 a}$ & $\mathbf{2 b}$ & $\mathbf{2 c}$ & $\mathbf{2 d}$ & $\mathbf{2 e}$ & $\mathbf{2 f}$ \\
\hline C3-C2-C1'-C2' & 126 & -82 & 17 & -89 & 30 & 133 \\
C2-C1'-C2'-C3' & 126 & -118 & 94 & 115 & -120 & -101 \\
\hline
\end{tabular}

Table A.9: Calculated ORD values for the six lowest energy conformers of $(2 S, 3 R)$ of naphth 2 at different wavelengths. All calculations were performed at the B3LYP/6-31G(d) level using the IEFPCM solvent continuum model with methanol as the solvent.

\begin{tabular}{lllll}
\hline Conformer & Population (\%) & \multicolumn{3}{c}{$[\alpha]^{\mathrm{DFT}}(\mathrm{deg})$} \\
& & $\lambda=546 \mathrm{~nm}$ & $\lambda=578 \mathrm{~nm}$ & $\lambda=589 \mathrm{~nm}$ \\
\hline $\mathrm{2a}$ & 6.4 & +225.0 & +136.3 & +112.7 \\
$2 \mathrm{~b}$ & 26.2 & +246.5 & +202.8 & +189.5 \\
$2 \mathrm{c}$ & 15.4 & +276.0 & +205.9 & +185.8 \\
$2 \mathrm{~d}$ & 10.5 & -215.5 & -210.4 & -206.5 \\
$2 \mathrm{e}$ & 29.3 & +314.5 & +246.1 & +225.9 \\
$2 \mathrm{f}$ & 12.2 & +873.2 & +690.4 & +636.3 \\
\hline Conformational average & +297.5 & +227.7 & +207.5 \\
Experimental & +132.5 & +103.6 & +92.8 \\
\hline
\end{tabular}

Table A.10: Important dihedral angles (deg) of optimized predominant conformers naphth 1a-1f obtained with DFT at the B3LYP/6-31G(d) level using the IEFPCM solvent continuum model with methanol as the solvent.

\begin{tabular}{lrrrrrr}
\hline Dihedral & \multicolumn{6}{c}{ Dihedral angle $(\mathrm{deg})$} \\
& $\mathbf{1 a}$ & $\mathbf{1 b}$ & $\mathbf{1 c}$ & $\mathbf{1 d}$ & $\mathbf{1 e}$ & $\mathbf{1 f}$ \\
\hline C3-C2-C1'-C2' & 129 & -81 & 17 & -88 & 30 & 135 \\
C2-C1'-C2'-C3' & 125 & -118 & 93 & 116 & -120 & -102 \\
\hline
\end{tabular}


Table A.11: Calculated ORD values for the six lowest energy conformers of $(2 S, 3 R)$ of naphth 1 at different wavelengths. All calculations were performed at the B3LYP/6-31G(d) level using the IEFPCM solvent continuum model with methanol as the solvent.

\begin{tabular}{lcccc}
\hline Conformer & Population $(\%)$ & \multicolumn{3}{c}{$[\alpha]^{\mathrm{DFT}}(\mathrm{deg})$} \\
& & $\lambda=546 \mathrm{~nm}$ & $\lambda=578 \mathrm{~nm}$ & $\lambda=589 \mathrm{~nm}$ \\
\hline $1 \mathrm{a}$ & 5.5 & -190.5 & -191.5 & -185.4 \\
$1 \mathrm{~b}$ & 34.6 & 109.1 & 114.5 & 130.6 \\
$1 \mathrm{c}$ & 11.3 & -118.1 & -122.3 & -133.0 \\
$1 \mathrm{~d}$ & 11.8 & -319.6 & -333.0 & -370.3 \\
$1 \mathrm{e}$ & 30.3 & 60.1 & 66.2 & 86.9 \\
$1 \mathrm{f}$ & 6.6 & 293.9 & 318.1 & 399.4 \\
\hline \multicolumn{2}{l}{ Conformational average } & +13.8 & +17.0 & +28.9 \\
\multicolumn{2}{l}{ Experimental } & -3.0 & -11.3 & -9.0 \\
\hline
\end{tabular}

Table A.12: Conformational analysis of naphth 1 using DFT computations. Relative energy, relative zero point energy, and relative Gibbs free energy $\left(\mathrm{kcal} \cdot \mathrm{mol}^{-1}\right)$ were calculated at the B3LYP/6-31G(d) level using the IEFPCM solvent continuum model with methanol as the solvent. $298.15 \mathrm{~K}$ and $1 \mathrm{~atm}$ were considered in the calculation for the Gibbs free energy. Conformational distribution was calculated based on the Boltzmann distribution.

\begin{tabular}{llrlrlr}
\hline Conformer & $\begin{array}{c}\Delta E \\
\left(\mathrm{kcal} \cdot \mathrm{mol}^{-1}\right)\end{array}$ & $\begin{array}{c}p_{E} \\
(\%)\end{array}$ & $\begin{array}{c}\Delta E_{0} \\
\left(\mathrm{kcal} \cdot \mathrm{mol}^{-1}\right)\end{array}$ & $\begin{array}{r}p_{E_{0}} \\
(\%)\end{array}$ & $\begin{array}{c}\Delta G \\
\left(\mathrm{kcal} \cdot \mathrm{mol}^{-1}\right)\end{array}$ & $\begin{array}{c}p_{G} \\
(\%)\end{array}$ \\
\hline 1a & 0.84 & 7.0 & 0.85 & 6.9 & 1.09 & 5.5 \\
1b & 0.15 & 23.0 & 0.18 & 21.3 & 0.00 & 34.6 \\
1c & 0.45 & 13.8 & 0.44 & 13.8 & 0.66 & 11.3 \\
1d & 0.29 & 18.0 & 0.23 & 19.8 & 0.40 & 11.8 \\
1e & 0.00 & 29.6 & 0.00 & 29.1 & 0.08 & 30.3 \\
1f & 0.73 & 8.6 & 0.69 & 9.0 & 0.98 & 6.6 \\
\hline
\end{tabular}




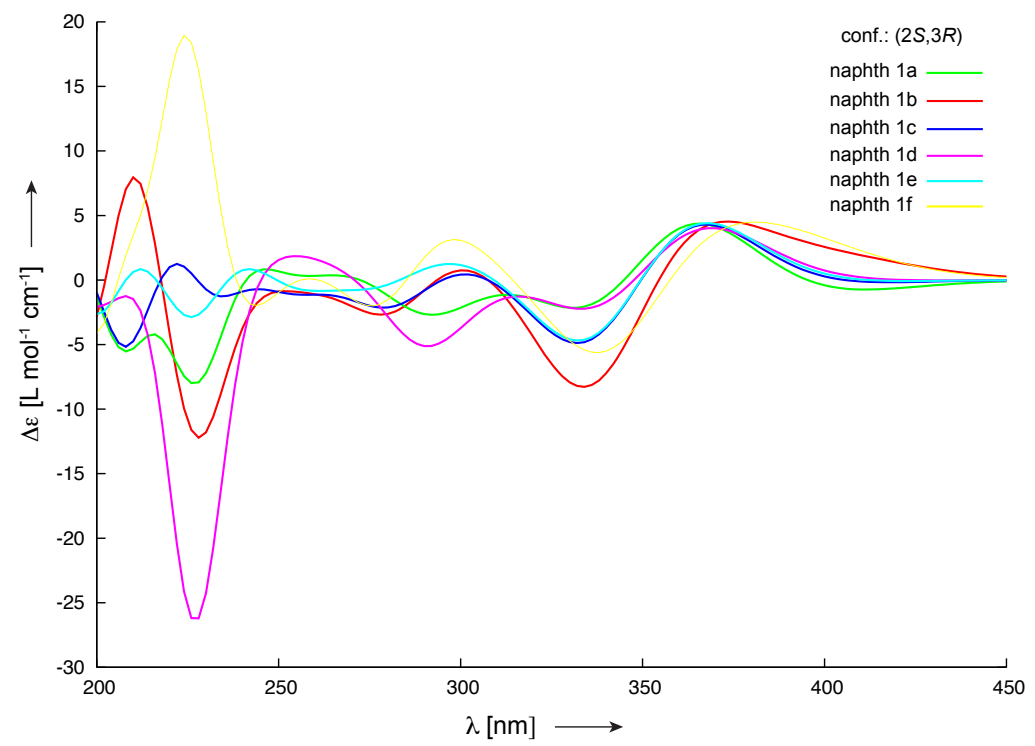

Figure A.8: Comparison of the computed ECD spectra for the six energy lowest conformers with a $(2 S, 3 R)$ configuration of naphth 1 . The calculations were performed with DFT at the B3LYP $/ 6-31 \mathrm{G}(\mathrm{d})$ level using the IEFPCM solvent continuum model with methanol as the solvent. 

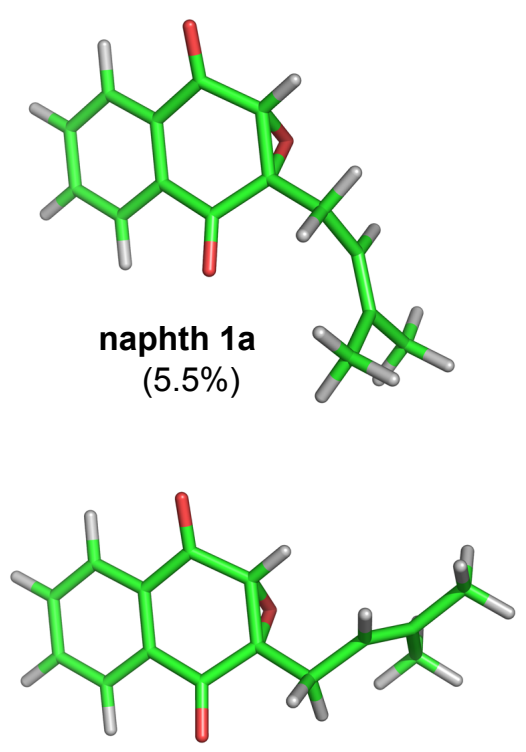

naphth 1c

$(11.3 \%)$

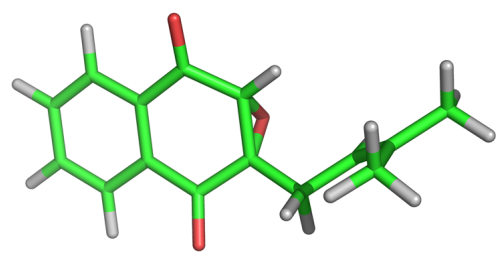

naphth 1e

$(30.3 \%)$
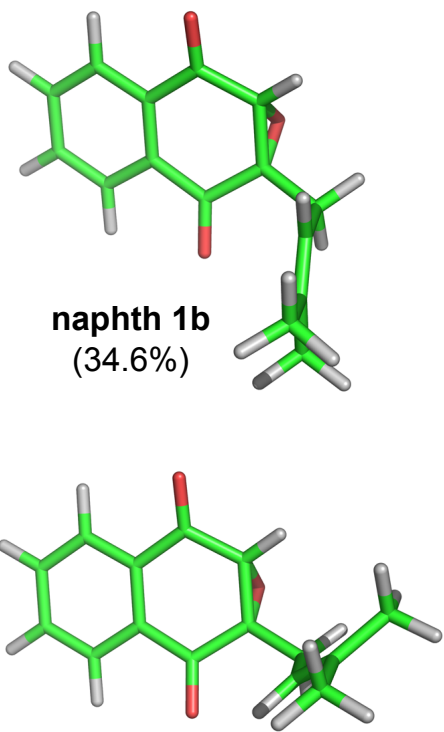

naphth 1d

$(11.8 \%)$

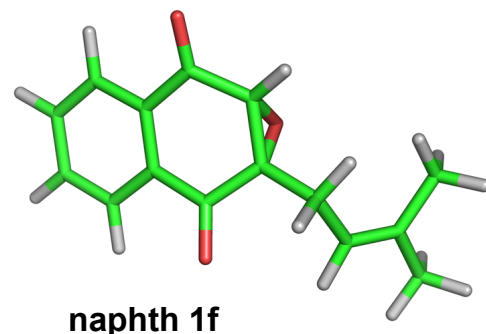

$(6.6 \%)$

Figure A.9: Six lowest energy conformers of (2S,3R)-2,3-epoxy-2,3-dihydrolapacho (naphth 1) with their populations calculated using the Gibbs free energy. All structures were optimized at the B3LYP/6-31G(d) level using the IEFPCM solvent continuum model with methanol as the solvent. 


\section{A.4 Additional data for the Michael addition prod- uct}
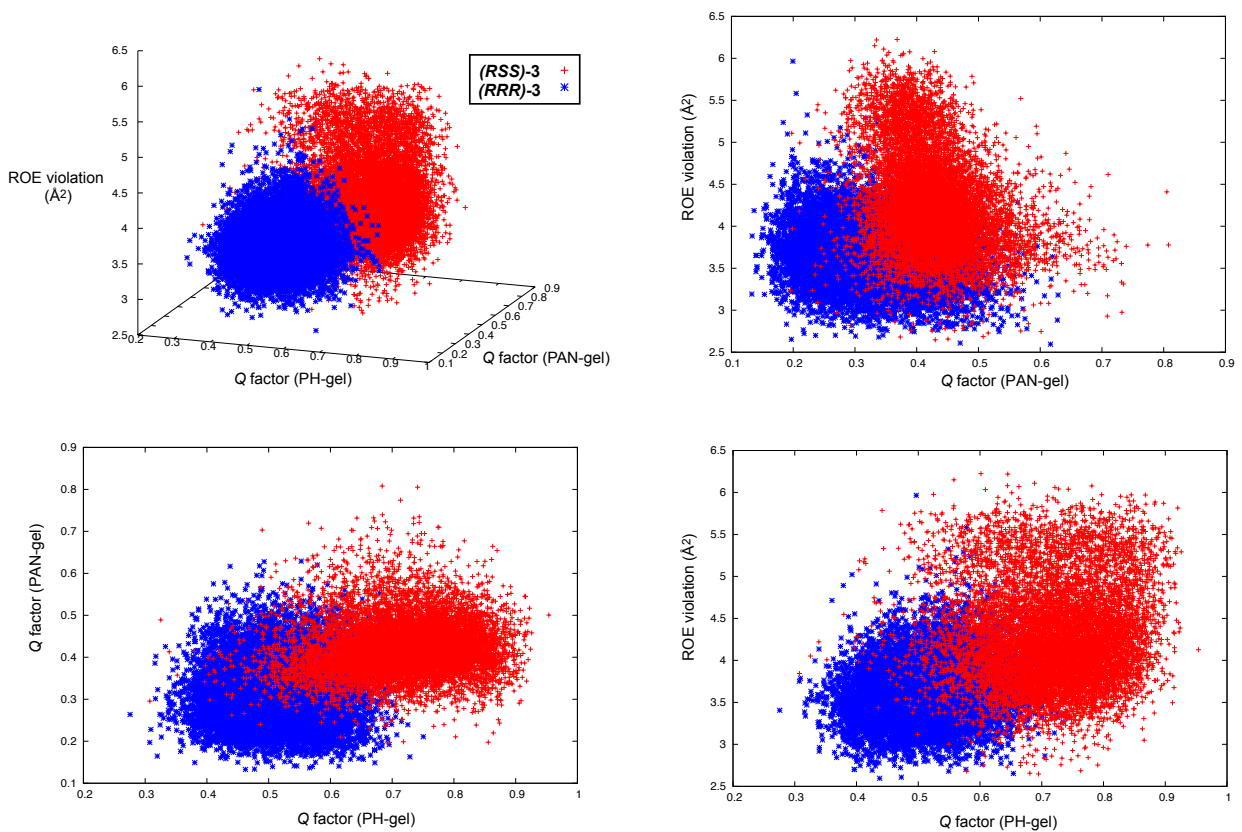

Figure A.10: The three 2D projections of Figure 5.4. 
Table A.13: Characteristics of the polymer media used to align $(R)$-3-anti.

\begin{tabular}{|c|c|c|c|}
\hline Medium & Polymer & Preparation & ${ }^{2} \mathrm{D}$ splitting $(\mathrm{Hz})$ \\
\hline PH-gel & Polyacrylamide & $\begin{array}{l}\text { Preparation follow- } \\
\text { ing [Haberz et al., } \\
\text { 2005], washed in HCl; } \\
\text { Monomer concentration } \\
0.75 \mathrm{~mol} / \mathrm{L} \text {; Gel cylinder } \\
\text { diameter } 5.4 \mathrm{~mm} \text {. }\end{array}$ & 6.5 \\
\hline PAN-gel & Polyacrylonitrile & $\begin{array}{l}\text { Preparation follow- } \\
\text { ing [Kummerlöwe et al., } \\
\text { 2007]; Monomer concen- } \\
\text { tration } 280 \mathrm{~g} / \mathrm{L} \text {; Irra- } \\
\text { diation with } 440 \text { kGy. } \\
\text { Polymer } \quad \text { molecular } \\
\text { weight: } 231000 \mathrm{~g} / \mathrm{mol} \text {. }\end{array}$ & 18.2 \\
\hline
\end{tabular}

Table A.14: Experimental and computed ORD values at different wavelengths. The computed ORD values are linearly weighted averages calculated from the best ensemble of the respective configuration, assuming that all conformations are equally populated. For all conformations, the $\mathrm{C}^{1}-\mathrm{N}$ bond is antiperiplanar. ORD computations were performed at the B3LYP/6-31G(d) level using the IEFPCM solvent continuum model with DMSO as the solvent.

\begin{tabular}{lrrrrrr}
\hline$\lambda(\mathrm{nm})$ & $\mathrm{T}$ & {$[\alpha]^{\exp }$} & \multicolumn{5}{c}{$[\alpha]^{\mathrm{DFT}}(\mathrm{deg})$} \\
$($ Source $)$ & $\left({ }^{\circ} \mathrm{C}\right)$ & $(\mathrm{deg})$ & $(R, R, R)-\mathbf{3}$ & $(R, S, S)-\mathbf{3}$ & $(S, S, S)-\mathbf{3}$ & $(S, R, R)-\mathbf{3}$ \\
\hline $589(\mathrm{Na})$ & 20.3 & -35 & -59 & 82 & 59 & -82 \\
$578(\mathrm{Hg})$ & 20.6 & -37 & -61 & 87 & 62 & -87 \\
$546(\mathrm{Hg})$ & 20.6 & -42 & -70 & 104 & 70 & -104 \\
$436(\mathrm{Hg})$ & 20.6 & -76 & -110 & 252 & 110 & -252 \\
\hline
\end{tabular}


Table A.15: Calculated ORD values of each conformation for the best ensemble $(R, R, R)$ 3 at different wavelengths. For all conformations, the $\mathrm{C}^{1}-\mathrm{N}$ bond is antiperiplanar. ORD calculations were performed at the B3LYP/6-31G(d) level using the IEFPCM solvent continuum model with DMSO as the solvent.

\begin{tabular}{lrrrr}
\hline$(R, R, R)-\mathbf{3}$ & \multicolumn{5}{c}{$[\alpha]^{\mathrm{DFT}}(\mathrm{deg})$} \\
& $\lambda=436 \mathrm{~nm}$ & $\lambda=546 \mathrm{~nm}$ & $\lambda=578 \mathrm{~nm}$ & $\lambda=589 \mathrm{~nm}$ \\
\hline 1 & -60 & -21 & -18 & -16 \\
2 & -60 & -22 & -17 & -17 \\
3 & 96 & 54 & 47 & 45 \\
4 & -470 & -262 & -230 & -219 \\
5 & -1362 & -749 & -654 & -622 \\
6 & -10 & 6 & 6 & 6 \\
7 & -11 & 5 & 6 & 6 \\
8 & -11 & 5 & 6 & 6 \\
9 & -58 & -20 & -16 & -15 \\
10 & 850 & 302 & 249 & 233 \\
\hline
\end{tabular}

Table A.16: Calculated ORD values of each conformation for the best ensemble $(R, S, S)$ 3 at different wavelengths. For all conformations, the $\mathrm{C}^{1}-\mathrm{N}$ bond is antiperiplanar. ORD calculations were performed at the B3LYP/6-31G(d) level using the IEFPCM solvent continuum model with DMSO as the solvent.

\begin{tabular}{lrrrr}
\hline$(R, S, S)-\mathbf{3}$ & \multicolumn{4}{c}{$[\alpha]^{\mathrm{DFT}}(\mathrm{deg})$} \\
& $\lambda=436 \mathrm{~nm}$ & $\lambda=546 \mathrm{~nm}$ & $\lambda=578 \mathrm{~nm}$ & $\lambda=589 \mathrm{~nm}$ \\
\hline 1 & 545 & 184 & 150 & 139 \\
2 & 306 & 150 & 129 & 122 \\
3 & 306 & 150 & 129 & 122 \\
4 & 316 & 160 & 137 & 130 \\
5 & 536 & 184 & 150 & 140 \\
6 & 296 & 144 & 124 & 117 \\
7 & 298 & 146 & 125 & 118 \\
8 & 306 & 149 & 129 & 122 \\
9 & -421 & -243 & -214 & -204 \\
10 & 47 & 18 & 14 & 13 \\
\hline
\end{tabular}




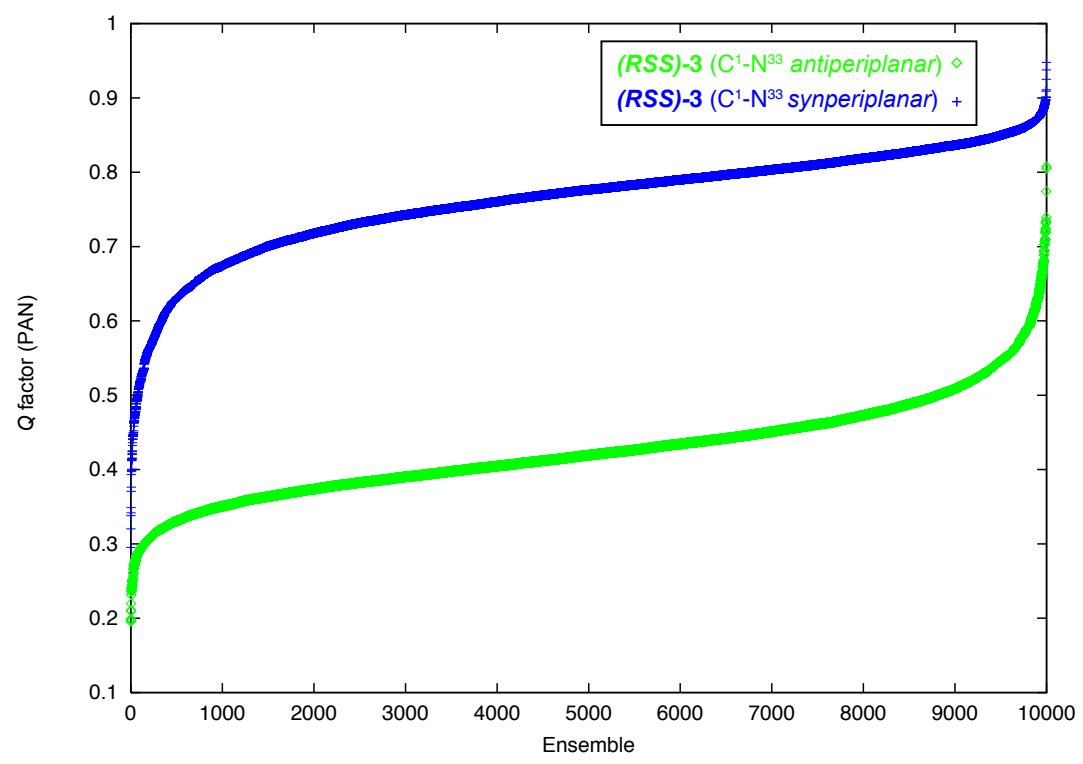

Figure A.11: Comparison of the $Q_{\mathrm{PAN}}$ factors between the $\mathrm{C}^{1} \mathrm{-N}$ antiperiplanar (green) and $\mathrm{C}^{1} \mathrm{-N}$ synperiplanar (blue) configurations of the diastereomer $(R, S, S)-\mathbf{3}$. 

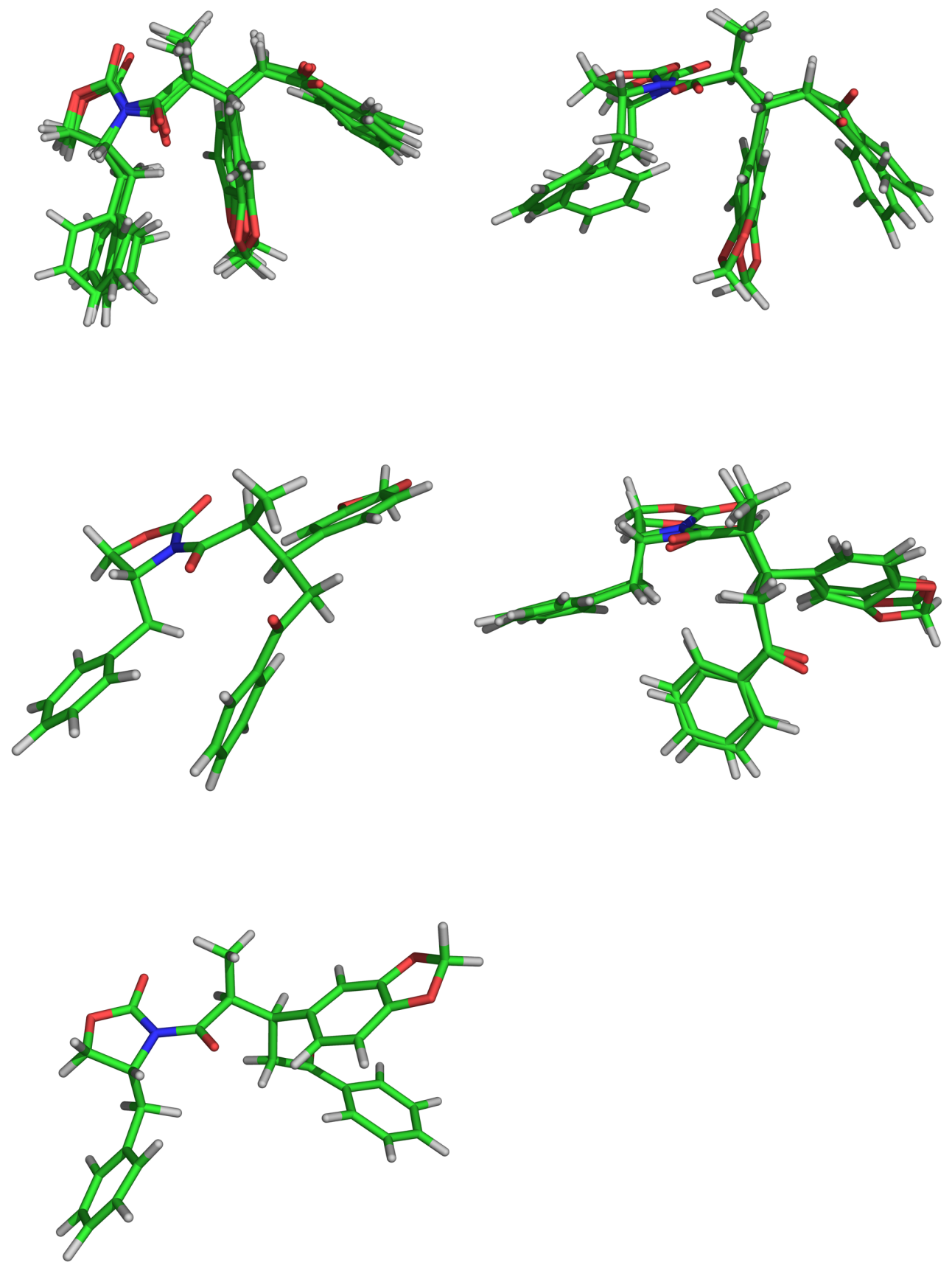

Figure A.12: The ten structures of the best ensemble of $(R, S, S)-\mathbf{3}$. For all conformations, the $\mathrm{C}^{1}-\mathrm{N}$ is antiperiplanar. All structures were optimized at the B3LYP/6-31G(d) level using the IEFPCM solvent continuum model with DMSO as the solvent. 


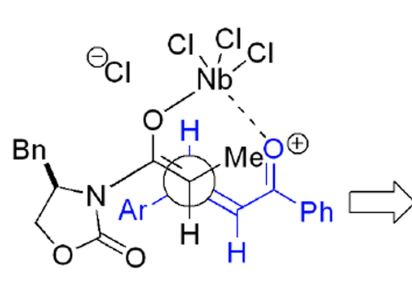

TS-A antiperiplanar

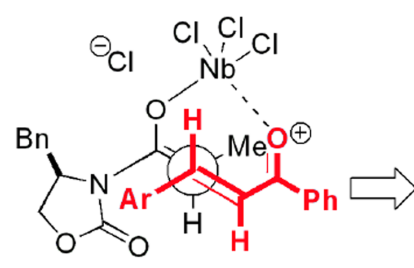

TS-C antiperiplanar<smiles>CCCC1[CH]COC(=O)N1C(=O)[C@H](C)[C@H](CC(=O)c1ccccc1)c1ccc2c(c1)OCO2</smiles>

$(R, R, R)-3$<smiles>C[C@H](C(=O)N1C(=O)OC[C@H]1Br)[Ge](CC(=O)c1ccccc1)c1ccc2c(c1)OCO2</smiles>

$(R, S, S)-3$
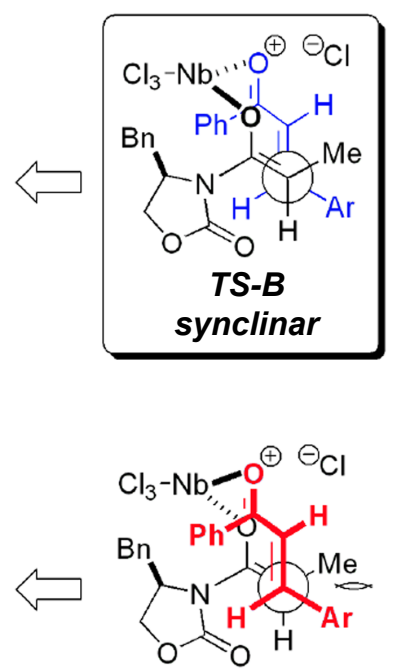

TS-D synclinar

Figure A.13: Four proposed transition states with antiperiplanar and synclinal attack on the $S i$ and $R e$ faces of the chalcone for both possible diastereomers $(R, R, R)-\mathbf{3}$ and $(R, S, S)-\mathbf{3}$. 


\section{A.5 Additional data for comp.540a}
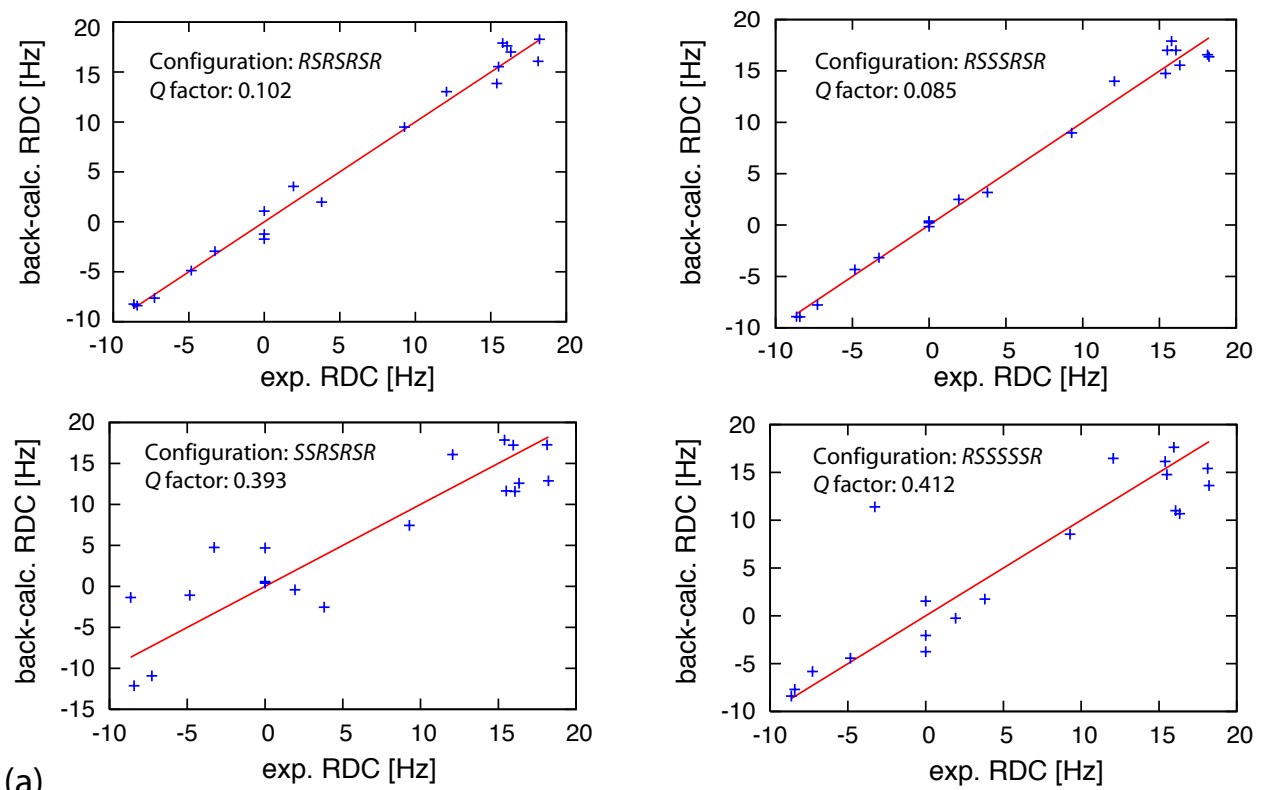

(a)
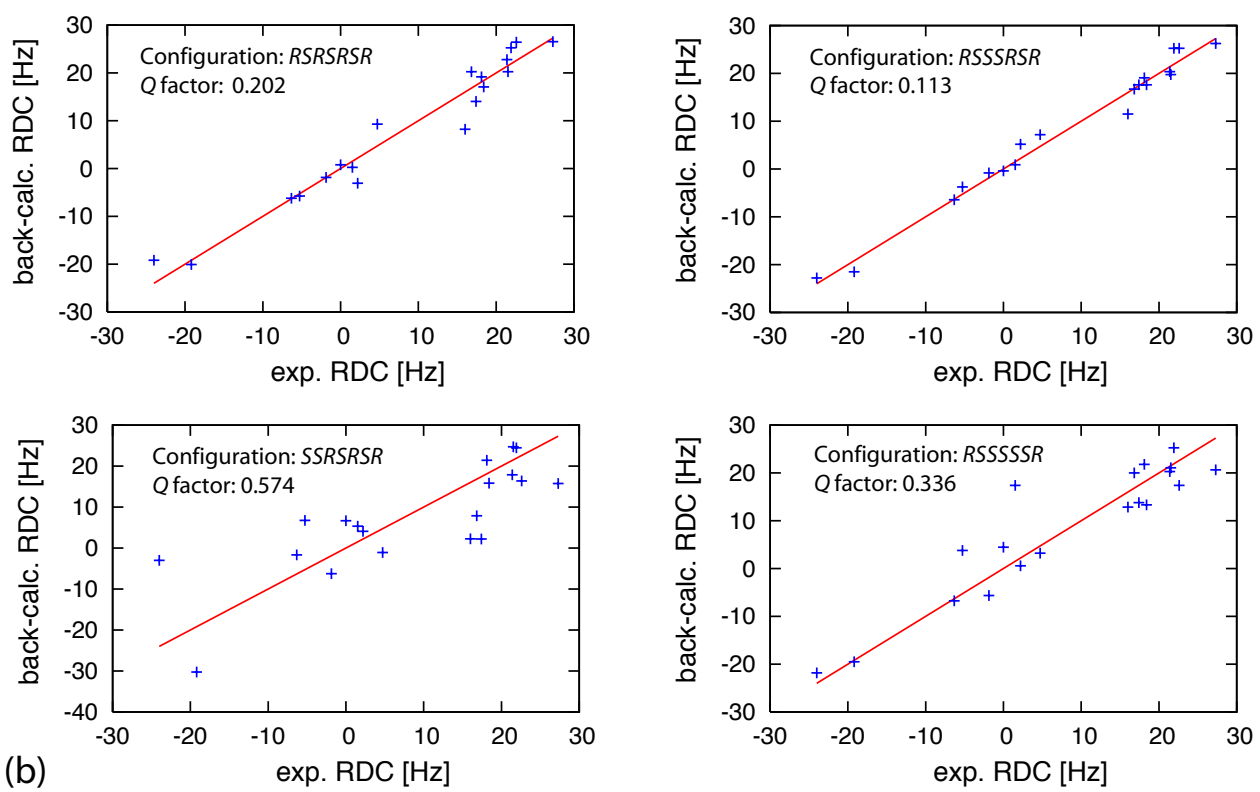

Figure A.14: Comparison of experimental and back-calculated RDCs of the remaining configurations selected from the MD simulation with floating chirality. The RDC fitting was performed using the experimental data of (a) PH-gel and (b) $(R)$-APhES-gel to a structure ensemble assuming a uniform alignment tensor for different conformations within one ensemble. 
Table A.17: Populations of the main configurations generated in the 40 ns restrained MD simulations with the floating chirality method. $10 \%, 15 \%$ and $20 \%$ of snapshots with lowest deviation energy were considered.

\begin{tabular}{|c|c|c|c|}
\hline & Percentage of snapshots & Configuration & Population \\
\hline \multirow[t]{7}{*}{ Simulation I } & \multirow[t]{2}{*}{$10 \%$} & $R S R S R S R$ & $45.5 \%$ \\
\hline & & $R S S S R S R$ & $54.5 \%$ \\
\hline & \multirow[t]{2}{*}{$15 \%$} & $R S R S R S R$ & $52.0 \%$ \\
\hline & & $R S S S R S R$ & $48.0 \%$ \\
\hline & \multirow[t]{3}{*}{$20 \%$} & $R S R S R S R$ & $61.6 \%$ \\
\hline & & $R S S S R S R$ & $36.8 \%$ \\
\hline & & $R S S S S S R$ & $1.6 \%$ \\
\hline \multirow[t]{9}{*}{ Simulation II } & \multirow[t]{3}{*}{$10 \%$} & $R S R S R S R$ & $50.0 \%$ \\
\hline & & $R S S S R S R$ & $33.3 \%$ \\
\hline & & $S S R S R S R$ & $16.7 \%$ \\
\hline & \multirow[t]{3}{*}{$15 \%$} & $R S R S R S R$ & $54.5 \%$ \\
\hline & & $R S S S R S R$ & $40.9 \%$ \\
\hline & & $S S R S R S R$ & $4.6 \%$ \\
\hline & \multirow[t]{3}{*}{$20 \%$} & $R S R S R S R$ & $39.0 \%$ \\
\hline & & $R S S S R S R$ & $56.3 \%$ \\
\hline & & $S S R S R S R$ & $4.7 \%$ \\
\hline \multirow[t]{6}{*}{ Simulation III } & $10 \%$ & $S R R R S R S$ & $100.0 \%$ \\
\hline & \multirow[t]{2}{*}{$15 \%$} & $S R R R S R S$ & $66.7 \%$ \\
\hline & & $S R R S R R S$ & $33.3 \%$ \\
\hline & \multirow[t]{3}{*}{$20 \%$} & $S R R R S R S$ & $61.5 \%$ \\
\hline & & $S R S R S R S$ & $23.1 \%$ \\
\hline & & $S R S R R R S$ & $15.4 \%$ \\
\hline
\end{tabular}


Table A.18: The $Q$ factors of the RDC fitting for different orientations between the lactone ring and the sugar. Two possible relative configurations were considered here: $\alpha$-Lrhamnose and $\alpha$-D-rhamnose, where the configuration of the lactone ring was maintained as $R S S S R S R$. The relative energy was calculated with force field based molecular mechanics calculations using the CFF force field.

\begin{tabular}{ccccc}
\hline Configuration & State & $\begin{array}{c}\text { Relative energy } \\
\left(\mathrm{kcal}^{-} \mathrm{mol}^{-1}\right)\end{array}$ & \multicolumn{2}{c}{$Q$ factor } \\
& & PH-gel & $(R)$-APhES-gel \\
\hline$\alpha$-L-rhamnose & 1 & 3.6 & 0.27 & 0.25 \\
& 2 & 9.6 & 0.39 & 0.35 \\
& 3 & 7.6 & 0.80 & 0.71 \\
& 4 & 3.7 & 0.42 & 0.56 \\
& 5 & 5.9 & 0.38 & 0.38 \\
& 6 & 0.0 & 0.28 & 0.39 \\
& 7 & 0.4 & 0.13 & 0.23 \\
& 8 & 7.0 & 0.46 & 0.54 \\
\hline \multirow{2}{*}{-D-rhamnose } & 1 & 3.3 & 0.36 & 0.30 \\
& 2 & 2.6 & 0.75 & 0.68 \\
& 3 & 0.0 & 0.26 & 0.32 \\
& 4 & 8.0 & 0.39 & 0.57 \\
& 5 & 3.6 & 0.42 & 0.41 \\
& 6 & 1.4 & 0.24 & 0.31 \\
& 7 & 2.0 & 0.44 & 0.50 \\
\hline
\end{tabular}




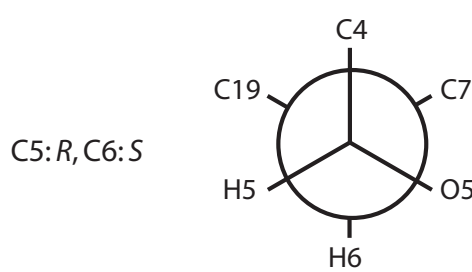

a

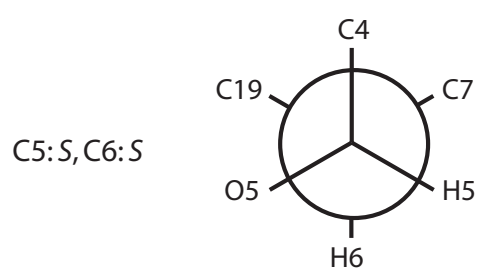

d

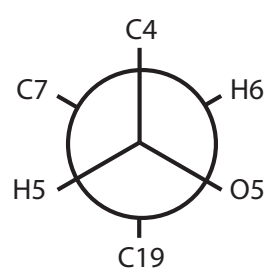

b

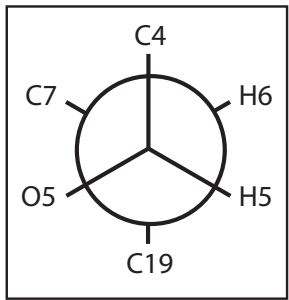

e

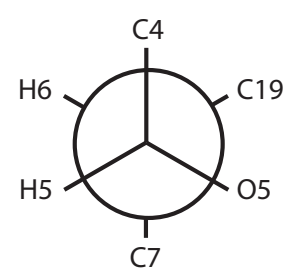

c

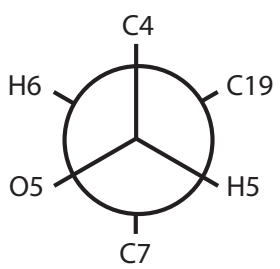

f

\begin{tabular}{lll}
\hline${ }^{3} J(\mathrm{H} 5-\mathrm{H} 6)$ & very small & no signal in COSY, no splitting in 1D signal \\
${ }^{2} J(\mathrm{H} 6-\mathrm{C} 5)$ & very small & no signal in $\left[{ }^{1} \mathrm{H}-{ }^{13} \mathrm{C}\right]-\mathrm{HSQMBC},\left[{ }^{1} \mathrm{H}-{ }^{13} \mathrm{C}\right]-\mathrm{HECADE}$ \\
${ }^{3} \mathrm{~J}(\mathrm{H} 5-\mathrm{C} 7)$ & $7 \mathrm{~Hz}$ & extracted from $\left[{ }^{1} \mathrm{H}-{ }^{13} \mathrm{C}\right]-\mathrm{HSQMBC}$ \\
\hline
\end{tabular}

Figure A.15: Newman projections of 6 possible conformers $(\mathbf{a}-\mathbf{f})$ for the dihedral C5-C6 of comp.540a. The three conformers on top have a configuration of $(5 R, 6 S)$, whereas the three conformers at the bottom have a configuration of $(5 S, 6 S)$. A very small ${ }^{3} J(\mathrm{H} 5$ $\mathrm{H} 6$ ) coupling excludes the conformers $\mathbf{b}$ and $\mathbf{f}$ as the main conformation. A very small ${ }^{2} J$ (H6-C5) coupling and a large ${ }^{3} J(\mathrm{H} 5-\mathrm{C} 7)$ coupling determines conformer e as the main conformation. The relative configuration of $\mathrm{C} 5$ and $\mathrm{C} 6$ was established as $(5 S, 6 S)$ or $(5 R, 6 R)$. 


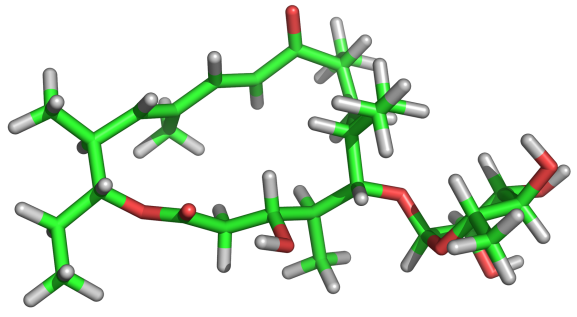

state 1
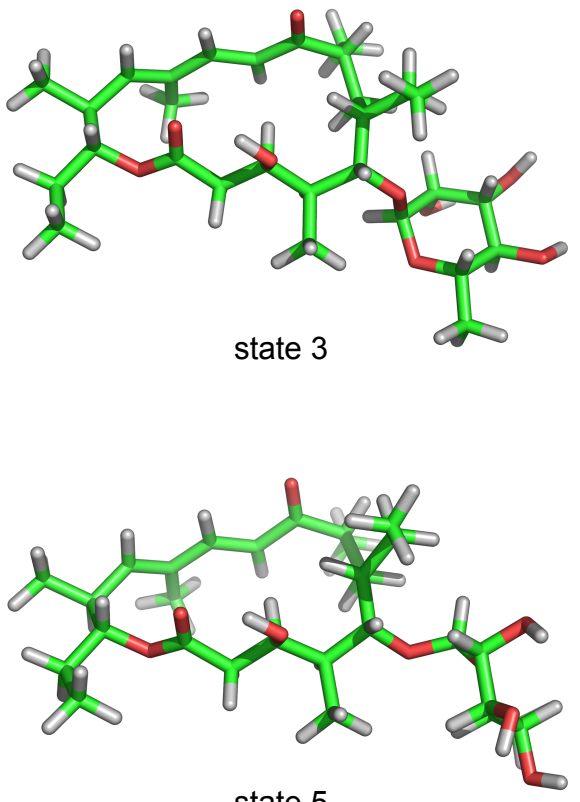

state 5

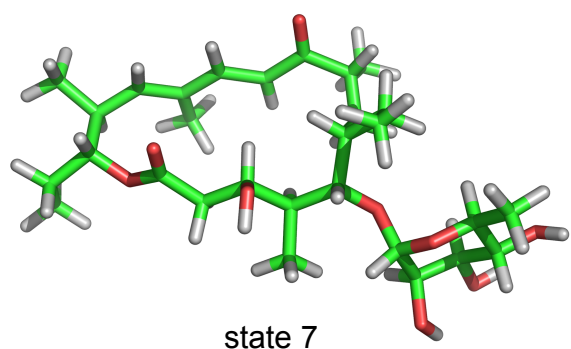

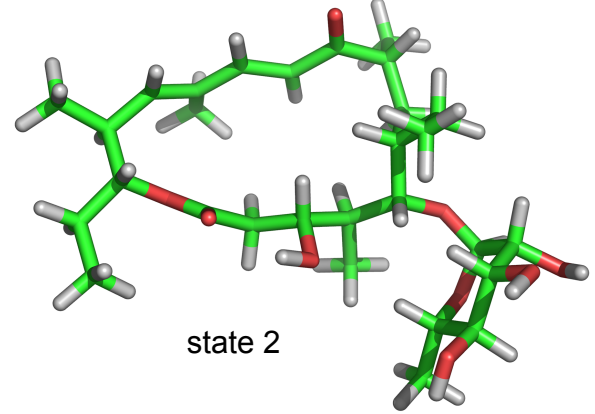
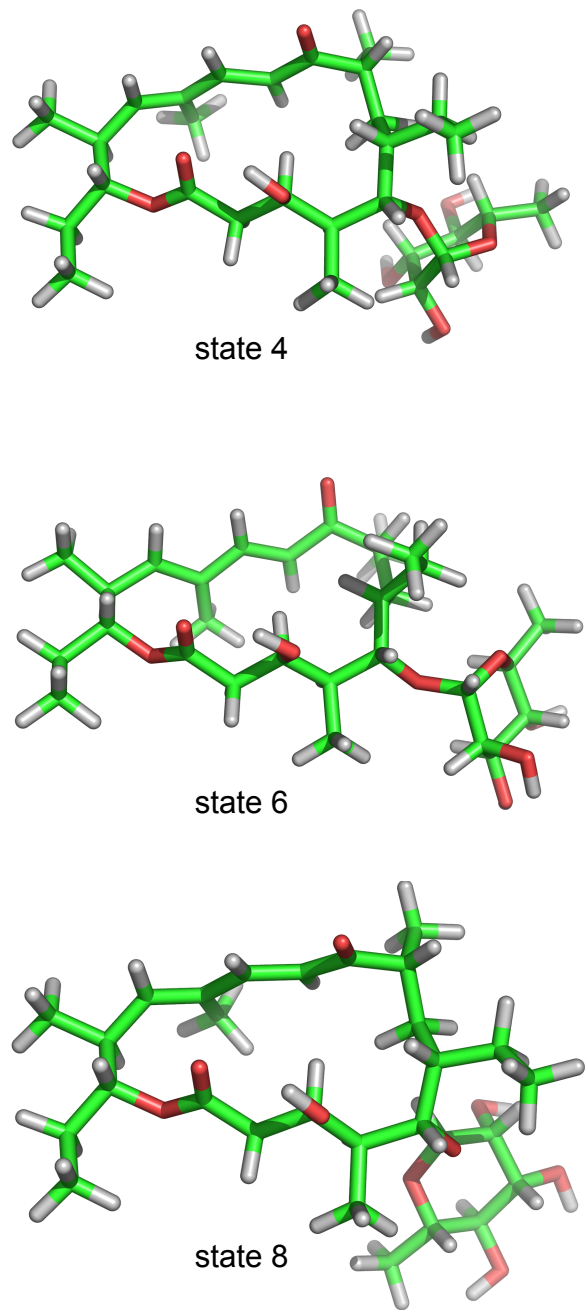

Figure A.16: Eight possible conformations of comp.540a with different orientations between macrolide and the $\alpha$-L-rhamnose. All structures have a configuration of $R S S S R S R$ in the lactone ring and they were minimized using the CFF force field. 


\section{A.6 Additional data for fibrosterol sulfate A}

Table A.19: Multiple alignment tensor fit: the dihedrals of each rotatable bond in the linker for each main conformation derived from RDC analysis (see Figure 6.3).

\begin{tabular}{lrrrr}
\hline \multirow{2}{*}{ Dihedral } & \multicolumn{4}{c}{ Dihedral angle } \\
& Conf. 1 & Conf. 2 & Conf. 3 & Conf. 4 \\
\hline H17-C17-C20-H20 & $113.69^{\circ}$ & $-176.23^{\circ}$ & $83.97^{\circ}$ & $94.95^{\circ}$ \\
H20-C20-C22-H22 & $-70.93^{\circ}$ & $-55.40^{\circ}$ & $175.62^{\circ}$ & $-65.22^{\circ}$ \\
H22-C22-C23-H23 & $-178.29^{\circ}$ & $-151.65^{\circ}$ & $173.69^{\circ}$ & $-148.21^{\circ}$ \\
H24-C24-C25-C26 & $12.87^{\circ}$ & $-163.05^{\circ}$ & $92.69^{\circ}$ & $78.43^{\circ}$ \\
H24'-C24'-C23'-C22' & $40.92^{\circ}$ & $54.67^{\circ}$ & $18.52^{\circ}$ & $30.92^{\circ}$ \\
C24'-C23'-C22'-C20' $^{\circ} 166.06^{\circ}$ & $173.01^{\circ}$ & $168.97^{\circ}$ & $-159.55^{\circ}$ \\
C23'-C22'-C20'-C17' $^{\circ}$ & $-174.72^{\circ}$ & $-162.42^{\circ}$ & $-145.99^{\circ}$ & $177.93^{\circ}$ \\
C22'-H20'C17'-H17' $^{\prime}$ & $-71.05^{\circ}$ & $-57.43^{\circ}$ & $-147.81^{\circ}$ & $-150.97^{\circ}$ \\
\hline
\end{tabular}


Table A.20: Single alignment tensor fit: number of remaining conformations after the filtering step using dihedral restraints derived from ${ }^{3} J_{\mathrm{HH}}$ couplings.

\begin{tabular}{lc}
\hline Configuration & Number of remaining conformations \\
\hline$R S R$ & 15 \\
$R R S$ & 14 \\
$S S R$ & 14 \\
$S R S$ & 41 \\
$R R R$ & 19 \\
$R S S$ & 21 \\
$S R R$ & 30 \\
$S S S$ & 24 \\
\hline
\end{tabular}

Table A.21: Single alignment tensor fit: The populations of the conformations and $Q$ factors of each configuration using the RDC fitting to a structure ensemble assuming a single alignment tensor for different conformations within one ensemble. The relative orientation of conformers in the ensemble is determined by superimposing each structure to one steroid ring system A'B'C'D'.

\begin{tabular}{lllll}
\hline Configuration & \multicolumn{3}{c}{ Population } & $Q$ factor \\
& Conf. 1 & Conf. 2 & Conf. 3 & \\
\hline$R S R$ & 0.71 & 0.18 & 0.12 & 0.42 \\
$R R S$ & 0.88 & 0.05 & 0.07 & 0.40 \\
$S S R$ & 0.32 & 0.49 & 0.19 & 0.27 \\
$S R S$ & 0.68 & 0.32 & & 0.30 \\
$R R R$ & 0.25 & 0.38 & 0.37 & 0.41 \\
$R S S$ & 0.66 & 0.15 & 0.19 & 0.29 \\
$S R R$ & 0.23 & 0.77 & & 0.41 \\
$S S S$ & 0.35 & 0.40 & 0.26 & 0.23 \\
\hline
\end{tabular}


Experimental RDCs versus back-calculated RDCs with an ensemble number of 4

(a)

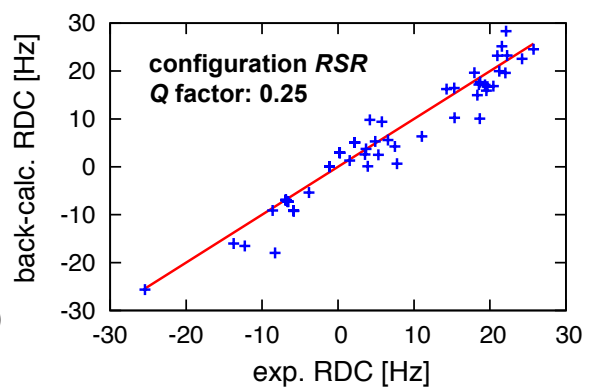

Experimental RDCs versus back-calculated RDCs with an ensemble number of 4

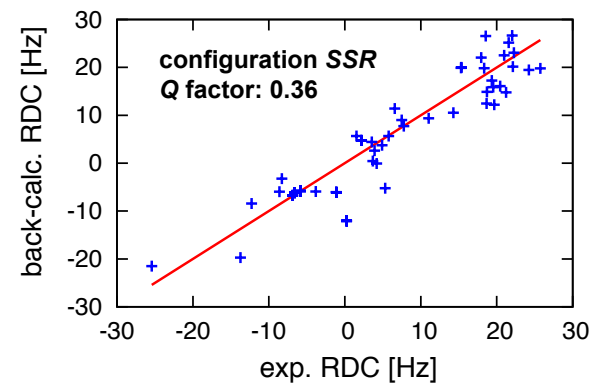

Experimental RDCs versus back-calculated RDCs with an ensemble number of 4

(e)

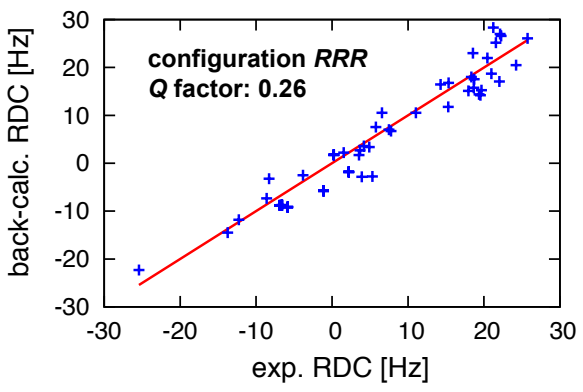

Experimental RDCs versus back-calculated RDCs with an ensemble number of 4

(g)

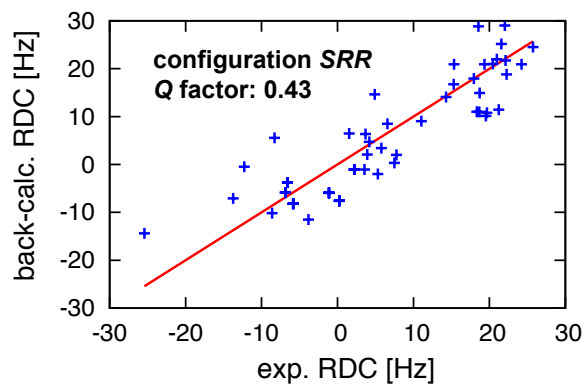

Experimental RDCs versus back-calculated RDCs with an ensemble number of 4

(b)

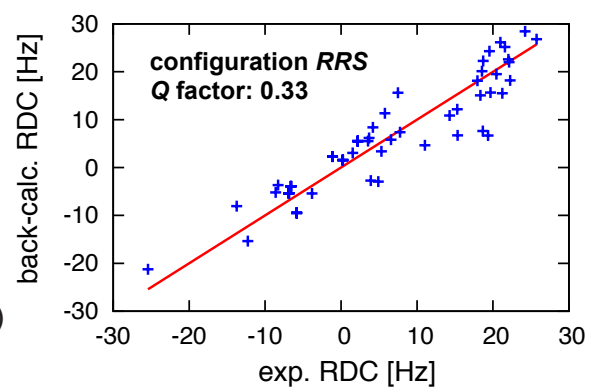

Experimental RDCs versus back-calculated RDCs with an ensemble number of 4

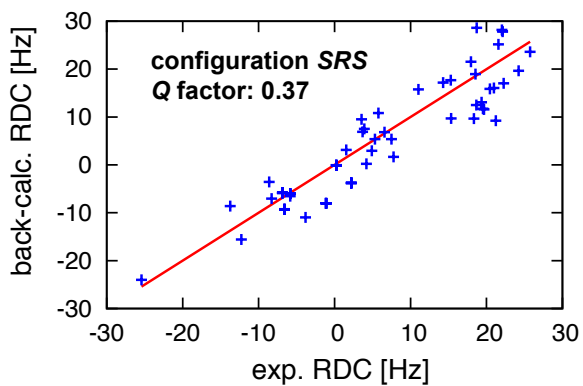

Experimental RDCs versus back-calculated RDCs with an ensemble number of 4

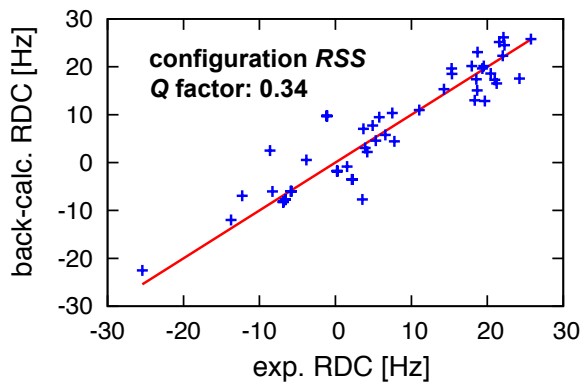

Experimental RDCs versus back-calculated RDCs with an ensemble number of 4

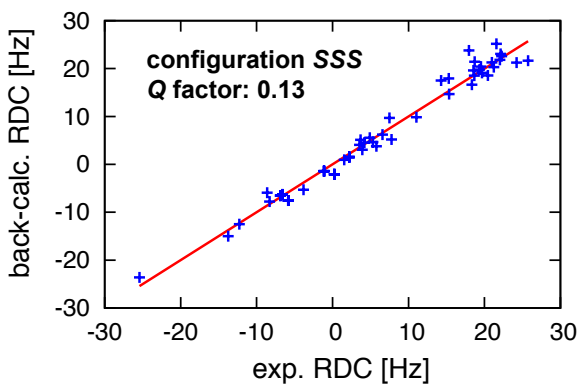

Figure A.17: Multiple alignment tensor fit: Comparison of the correlations of experimental and back-calculated RDCs of 8 possible configurations. The SVD fitting of the $44{ }^{1} D_{\mathrm{CH}}$ RDCs was performed against four conformers for each possible configuration. The four main conformers and the populations were obtained from a selected unrestrained MD simulation based on NOE and ${ }^{3} J$ couplings. The alignment tensor was determined individually for each conformer. 


\begin{tabular}{|c|c|c|c|c|c|c|c|c|c|c|c|}
\hline & \multicolumn{5}{|c|}{ Conformer 1} & \multicolumn{5}{|c|}{ Conformer 2} \\
\hline & & $\mathbf{A}_{\mathrm{xy}}$ & $\mathbf{A}_{\mathrm{xz}}$ & $\mathbf{A}_{\mathbf{y y}}$ & $\mathbf{A}_{\mathbf{y z}}$ & $\overline{\mathbf{A}_{\mathrm{zz}}}$ & $\mathbf{A}_{\mathbf{x y}}$ & $\mathbf{A}_{\mathrm{xz}}$ & $\mathbf{A}_{\mathbf{y y}}$ & $\mathbf{A}_{\mathbf{y z}}$ & $\overline{\mathbf{A}_{\mathrm{zz}}}$ \\
\hline \multirow{5}{*}{$\stackrel{\bar{\theta}}{\underline{\theta}}$} & $\mathbf{A}_{\mathrm{xy}}$ & $3.9 \mathrm{E}-09$ & $-5.6 \mathrm{E}-10$ & $-5.4 \mathrm{E}-10$ & $-8.4 \mathrm{E}-10$ & $2.5 \mathrm{E}-10$ & $2.7 \mathrm{E}-10$ & $-3.1 \mathrm{E}-09$ & $-3.7 \mathrm{E}-09$ & $-8.1 \mathrm{E}-09$ & $1.2 \mathrm{E}-10$ \\
\hline & $\mathbf{A}_{\mathbf{x z}}$ & $-5.6 \mathrm{E}-10$ & $2.4 \mathrm{E}-09$ & $8.0 \mathrm{E}-11$ & $-9.8 \mathrm{E}-12$ & $-2.4 \mathrm{E}-10$ & $-1.0 \mathrm{E}-10$ & $1.9 \mathrm{E}-09$ & $1.3 \mathrm{E}-09$ & $4.5 \mathrm{E}-09$ & $-3.1 \mathrm{E}-09$ \\
\hline & $\mathbf{A}_{\mathbf{y y}}$ & $-5.4 \mathrm{E}-10$ & $8.0 \mathrm{E}-11$ & $4.4 \mathrm{E}-09$ & $1.2 \mathrm{E}-09$ & $-2.2 \mathrm{E}-10$ & $-5.7 \mathrm{E}-09$ & $-5.3 \mathrm{E}-09$ & $2.2 \mathrm{E}-10$ & $3.7 \mathrm{E}-09$ & $2.9 \mathrm{E}-10$ \\
\hline & $\mathbf{A}_{\mathrm{yz}}$ & $-8.4 \mathrm{E}-10$ & $-9.8 \mathrm{E}-12$ & $1.2 \mathrm{E}-09$ & $5.6 \mathrm{E}-09$ & $-1.8 \mathrm{E}-09$ & $1.8 \mathrm{E}-09$ & $-2.0 \mathrm{E}-09$ & $-6.0 \mathrm{E}-09$ & $1.4 \mathrm{E}-09$ & $1.5 \mathrm{E}-08$ \\
\hline & $\mathbf{A}_{\mathrm{zz}}$ & $2.5 \mathrm{E}-10$ & $-2.4 \mathrm{E}-10$ & $-2.2 \mathrm{E}-10$ & $-1.8 \mathrm{E}-09$ & $3.1 \mathrm{E}-09$ & $-1.5 \mathrm{E}-09$ & $-7.0 \mathrm{E}-10$ & $5.5 \mathrm{E}-09$ & $-3.8 \mathrm{E}-09$ & $-2.1 \mathrm{E}-09$ \\
\hline \multirow{5}{*}{$\frac{N}{\vdots}$} & $\mathbf{A}_{\mathbf{x y}}$ & $2.7 \mathrm{E}-10$ & $-1.0 \mathrm{E}-10$ & $-5.7 \mathrm{E}-09$ & $1.8 \mathrm{E}-09$ & $-1.5 \mathrm{E}-09$ & $1.4 \mathrm{E}-08$ & $1.0 \mathrm{E}-08$ & $-6.4 \mathrm{E}-09$ & $-3.2 \mathrm{E}-09$ & $7.1 \mathrm{E}-09$ \\
\hline & $\mathbf{A}_{\mathrm{xz}}$ & $-3.1 \mathrm{E}-09$ & $1.9 \mathrm{E}-09$ & $-5.3 \mathrm{E}-09$ & $-2.0 \mathrm{E}-09$ & $-7.0 \mathrm{E}-10$ & $1.0 \mathrm{E}-08$ & $2.7 \mathrm{E}-08$ & $1.4 \mathrm{E}-10$ & $1.5 \mathrm{E}-08$ & $-1.4 \mathrm{E}-08$ \\
\hline & $\mathbf{A}_{\mathbf{y y}}$ & $-3.7 \mathrm{E}-09$ & $1.3 \mathrm{E}-09$ & $2.2 \mathrm{E}-10$ & $-6.0 \mathrm{E}-09$ & $5.5 \mathrm{E}-09$ & $-6.4 \mathrm{E}-09$ & $1.4 \mathrm{E}-10$ & $2.7 \mathrm{E}-08$ & $5.3 \mathrm{E}-10$ & $-2.4 \mathrm{E}-08$ \\
\hline & $\mathbf{A}_{\mathbf{y z}}$ & $-8.1 \mathrm{E}-09$ & $4.5 \mathrm{E}-09$ & $3.7 \mathrm{E}-09$ & $1.4 \mathrm{E}-09$ & $-3.8 \mathrm{E}-09$ & $-3.2 \mathrm{E}-09$ & $1.5 \mathrm{E}-08$ & $5.3 \mathrm{E}-10$ & $5.7 \mathrm{E}-08$ & $-2.1 \mathrm{E}-08$ \\
\hline & $\mathbf{A}_{\mathrm{zz}}$ & $1.2 \mathrm{E}-10$ & $-3.1 \mathrm{E}-09$ & $2.9 \mathrm{E}-10$ & $1.5 \mathrm{E}-08$ & $-2.1 \mathrm{E}-09$ & 7.1E-09 & $-1.4 \mathrm{E}-08$ & $-2.4 \mathrm{E}-08$ & $-2.1 \mathrm{E}-08$ & $8.3 \mathrm{E}-08$ \\
\hline \multirow{5}{*}{$\stackrel{\infty}{\vdots}$} & $\mathbf{A}_{\mathbf{x y}}$ & $1.8 \mathrm{E}-10$ & $-1.1 \mathrm{E}-09$ & $-1.1 \mathrm{E}-08$ & $4.7 \mathrm{E}-09$ & $-3.4 \mathrm{E}-09$ & $2.2 \mathrm{E}-08$ & $2.0 \mathrm{E}-08$ & $-2.0 \mathrm{E}-08$ & $-2.9 \mathrm{E}-08$ & $6.0 \mathrm{E}-08$ \\
\hline & $\mathbf{A}_{\mathbf{x z}}$ & $-4.4 \mathrm{E}-09$ & $3.8 \mathrm{E}-09$ & $5.2 \mathrm{E}-09$ & $-1.6 \mathrm{E}-08$ & $3.0 \mathrm{E}-09$ & $-1.6 \mathrm{E}-08$ & $1.1 \mathrm{E}-08$ & $3.6 \mathrm{E}-08$ & $2.4 \mathrm{E}-08$ & $-8.7 \mathrm{E}-08$ \\
\hline & $\mathbf{A}_{\mathbf{y y}}$ & $1.9 \mathrm{E}-11$ & $-1.7 \mathrm{E}-10$ & $8.4 \mathrm{E}-09$ & $-6.1 \mathrm{E}-09$ & $5.7 \mathrm{E}-09$ & $-1.7 \mathrm{E}-08$ & $-2.3 \mathrm{E}-08$ & $2.3 \mathrm{E}-08$ & $-1.5 \mathrm{E}-08$ & $-1.2 \mathrm{E}-08$ \\
\hline & $\mathbf{A}_{\mathbf{y z}}$ & $1.3 \mathrm{E}-08$ & $-8.1 \mathrm{E}-09$ & $-7.9 \mathrm{E}-09$ & $1.0 \mathrm{E}-08$ & $-2.9 \mathrm{E}-09$ & $2.0 \mathrm{E}-08$ & $-1.8 \mathrm{E}-08$ & $-4.0 \mathrm{E}-08$ & $-8.5 \mathrm{E}-08$ & $8.7 \mathrm{E}-08$ \\
\hline & $\mathbf{A}_{\mathrm{zz}}$ & $-4.2 \mathrm{E}-09$ & $2.8 \mathrm{E}-09$ & $-7.4 \mathrm{E}-09$ & $1.3 \mathrm{E}-08$ & $-2.0 \mathrm{E}-09$ & $2.0 \mathrm{E}-08$ & $1.6 \mathrm{E}-08$ & $-1.7 \mathrm{E}-08$ & $-1.0 \mathrm{E}-09$ & $6.5 \mathrm{E}-08$ \\
\hline \multirow{5}{*}{$\frac{\nabla}{\stackrel{\Xi}{\Xi}}$} & $\mathbf{A}_{\mathbf{x y}}$ & $-1.7 \mathrm{E}-09$ & $-2.7 \mathrm{E}-09$ & $-9.4 \mathrm{E}-10$ & $-1.7 \mathrm{E}-08$ & $1.2 \mathrm{E}-08$ & $-1.8 \mathrm{E}-08$ & $-1.5 \mathrm{E}-08$ & $4.5 \mathrm{E}-08$ & $-2.5 \mathrm{E}-08$ & $-4.2 \mathrm{E}-08$ \\
\hline & $\mathbf{A}_{\mathrm{xz}}$ & $-7.1 \mathrm{E}-09$ & $-1.5 \mathrm{E}-09$ & $2.9 \mathrm{E}-08$ & $4.1 \mathrm{E}-09$ & $3.9 \mathrm{E}-09$ & $-5.3 \mathrm{E}-08$ & $-7.0 \mathrm{E}-08$ & $2.5 \mathrm{E}-08$ & $1.3 \mathrm{E}-08$ & $2.1 \mathrm{E}-08$ \\
\hline & $\mathbf{A}_{\mathbf{y y}}$ & $-3.4 \mathrm{E}-09$ & $4.7 \mathrm{E}-09$ & $-1.4 \mathrm{E}-08$ & $1.6 \mathrm{E}-09$ & $-4.7 \mathrm{E}-09$ & $2.5 \mathrm{E}-08$ & 4.3E-08 & $-1.5 \mathrm{E}-08$ & $7.0 \mathrm{E}-09$ & $5.2 \mathrm{E}-09$ \\
\hline & $\mathbf{A}_{\mathbf{y z}}$ & $-5.1 \mathrm{E}-09$ & $3.9 \mathrm{E}-09$ & $1.1 \mathrm{E}-08$ & $-4.1 \mathrm{E}-09$ & $1.5 \mathrm{E}-09$ & $-2.3 \mathrm{E}-08$ & $-1.7 \mathrm{E}-08$ & $1.5 \mathrm{E}-08$ & $4.3 \mathrm{E}-08$ & $-4.7 \mathrm{E}-08$ \\
\hline & $\mathbf{A}_{\mathrm{zz}}$ & $4.7 \mathrm{E}-10$ & $3.0 \mathrm{E}-09$ & $2.0 \mathrm{E}-09$ & $-5.6 \mathrm{E}-09$ & $-1.7 \mathrm{E}-09$ & $-8.1 \mathrm{E}-09$ & $2.5 \mathrm{E}-10$ & $2.8 \mathrm{E}-10$ & $1.3 \mathrm{E}-08$ & $-3.5 \mathrm{E}-08$ \\
\hline
\end{tabular}

Figure A.18: Multiple alignment tensor fit: variance-covariance matrix of the alignment tensor parameters of the best-fitting configuration $S S S$, calculated from Equation 6.1 (Chapter 6). The variance of each tensor data is depicted in red. 


\begin{tabular}{|c|c|c|c|c|c|c|c|c|c|c|c|}
\hline & & \multicolumn{5}{|c|}{ Conformer 3} & \multicolumn{5}{|c|}{ Conformer 4} \\
\hline & & $\mathbf{A}_{x y}$ & $\mathbf{A}_{\mathbf{x z}}$ & $\mathbf{A}_{\mathbf{y y}}$ & $\mathbf{A}_{\mathbf{y z}}$ & $\mathbf{A}_{\mathrm{zz}}$ & $\mathbf{A}_{\mathbf{x y}}$ & $\mathbf{A}_{\mathrm{xz}}$ & $\mathbf{A}_{\mathbf{y y}}$ & $\mathbf{A}_{\mathbf{y z}}$ & $\mathbf{A}_{\mathrm{zz}}$ \\
\hline \multirow{5}{*}{$\stackrel{\bar{g}}{\underline{0}}$} & $\mathbf{A}_{\mathbf{x y}}$ & $1.8 \mathrm{E}-10$ & $-4.4 \mathrm{E}-09$ & $1.9 \mathrm{E}-11$ & $1.3 \mathrm{E}-08$ & $-4.2 \mathrm{E}-09$ & $-1.7 \mathrm{E}-09$ & $-7.1 \mathrm{E}-09$ & $-3.4 \mathrm{E}-09$ & $-5.1 \mathrm{E}-09$ & $4.7 \mathrm{E}-10$ \\
\hline & $\mathbf{A}_{\mathrm{xz}}$ & $-1.1 \mathrm{E}-09$ & $3.8 \mathrm{E}-09$ & $-1.7 \mathrm{E}-10$ & $-8.1 \mathrm{E}-09$ & $2.8 \mathrm{E}-09$ & $-2.7 \mathrm{E}-09$ & $-1.5 \mathrm{E}-09$ & $4.7 \mathrm{E}-09$ & $3.9 \mathrm{E}-09$ & $3.0 \mathrm{E}-09$ \\
\hline & $\mathbf{A}_{\mathbf{y y}}$ & $-1.1 \mathrm{E}-08$ & $5.2 \mathrm{E}-09$ & $8.4 \mathrm{E}-09$ & $-7.9 \mathrm{E}-09$ & $-7.4 \mathrm{E}-09$ & $-9.4 \mathrm{E}-10$ & $2.9 \mathrm{E}-08$ & $-1.4 \mathrm{E}-08$ & $1.1 \mathrm{E}-08$ & $2.0 \mathrm{E}-09$ \\
\hline & $\mathbf{A}_{\mathbf{y z}}$ & $4.7 \mathrm{E}-09$ & $-1.6 \mathrm{E}-08$ & $-6.1 \mathrm{E}-09$ & $1.0 \mathrm{E}-08$ & $1.3 \mathrm{E}-08$ & $-1.7 \mathrm{E}-08$ & $4.1 \mathrm{E}-09$ & $1.6 \mathrm{E}-09$ & $-4.1 \mathrm{E}-09$ & $-5.6 \mathrm{E}-09$ \\
\hline & $\mathbf{A}_{\mathrm{zz}}$ & $-3.4 \mathrm{E}-09$ & $3.0 \mathrm{E}-09$ & $5.7 \mathrm{E}-09$ & $-2.9 \mathrm{E}-09$ & $-2.0 \mathrm{E}-09$ & $1.2 \mathrm{E}-08$ & $3.9 \mathrm{E}-09$ & $-4.7 \mathrm{E}-09$ & $1.5 \mathrm{E}-09$ & $-1.7 \mathrm{E}-09$ \\
\hline \multirow{5}{*}{ 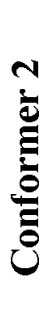 } & $\mathbf{A}_{\mathbf{x y}}$ & $2.2 \mathrm{E}-08$ & $-1.6 \mathrm{E}-08$ & $-1.7 \mathrm{E}-08$ & $2.0 \mathrm{E}-08$ & $2.0 \mathrm{E}-08$ & $-1.8 \mathrm{E}-08$ & $-5.3 \mathrm{E}-08$ & $2.5 \mathrm{E}-08$ & $-2.3 \mathrm{E}-08$ & $-8.1 \mathrm{E}-09$ \\
\hline & $\mathbf{A}_{\mathrm{xz}}$ & $2.0 \mathrm{E}-08$ & $1.1 \mathrm{E}-08$ & $-2.3 \mathrm{E}-08$ & $-1.8 \mathrm{E}-08$ & $1.6 \mathrm{E}-08$ & $-1.5 \mathrm{E}-08$ & $-7.0 \mathrm{E}-08$ & $4.3 \mathrm{E}-08$ & $-1.7 \mathrm{E}-08$ & $2.5 \mathrm{E}-10$ \\
\hline & $\mathbf{A}_{\mathbf{y y}}$ & $-2.0 \mathrm{E}-08$ & $3.6 \mathrm{E}-08$ & $2.3 \mathrm{E}-08$ & $-4.0 \mathrm{E}-08$ & $-1.7 \mathrm{E}-08$ & $4.5 \mathrm{E}-08$ & $2.5 \mathrm{E}-08$ & $-1.5 \mathrm{E}-08$ & $1.5 \mathrm{E}-08$ & $2.8 \mathrm{E}-10$ \\
\hline & $\mathbf{A}_{\mathbf{y z}}$ & $-2.9 \mathrm{E}-08$ & $2.4 \mathrm{E}-08$ & $-1.5 \mathrm{E}-08$ & $-8.5 \mathrm{E}-08$ & $-1.0 \mathrm{E}-09$ & $-2.5 \mathrm{E}-08$ & $1.3 \mathrm{E}-08$ & $7.0 \mathrm{E}-09$ & $4.3 \mathrm{E}-08$ & $1.3 \mathrm{E}-08$ \\
\hline & $\mathbf{A}_{\mathrm{zz}}$ & $6.0 \mathrm{E}-08$ & $-8.7 \mathrm{E}-08$ & $-1.2 \mathrm{E}-08$ & $8.7 \mathrm{E}-08$ & $6.5 \mathrm{E}-08$ & $-4.2 \mathrm{E}-08$ & $2.1 \mathrm{E}-08$ & $5.2 \mathrm{E}-09$ & $-4.7 \mathrm{E}-08$ & $-3.5 \mathrm{E}-08$ \\
\hline \multirow{5}{*}{$\stackrel{m}{\stackrel{0}{\sigma}}$} & $\mathbf{A}_{\mathbf{x y}}$ & $1.4 \mathrm{E}-07$ & $-6.6 E-08$ & $-3.7 \mathrm{E}-08$ & $1.0 \mathrm{E}-07$ & $8.0 \mathrm{E}-08$ & $-2.4 \mathrm{E}-08$ & $-9.6 \mathrm{E}-08$ & 7.6E- 08 & $-1.1 \mathrm{E}-07$ & $-3.9 \mathrm{E}-08$ \\
\hline & $\mathbf{A}_{\mathrm{xz}}$ & $-6.6 \mathrm{E}-08$ & $1.2 \mathrm{E}-07$ & $4.1 \mathrm{E}-08$ & $-1.1 \mathrm{E}-07$ & $-7.5 \mathrm{E}-08$ & $5.7 \mathrm{E}-08$ & $2.6 \mathrm{E}-08$ & $-1.2 \mathrm{E}-08$ & $5.5 \mathrm{E}-08$ & $4.3 \mathrm{E}-08$ \\
\hline & $\mathbf{A}_{\mathbf{y y}}$ & $-3.7 \mathrm{E}-08$ & $4.1 \mathrm{E}-08$ & $8.4 \mathrm{E}-08$ & 7.4E-09 & $-2.6 \mathrm{E}-08$ & $4.4 \mathrm{E}-08$ & $1.1 \mathrm{E}-07$ & $-6.0 \mathrm{E}-08$ & $3.2 \mathrm{E}-08$ & $1.2 \mathrm{E}-08$ \\
\hline & $\mathbf{A}_{\mathbf{y z}}$ & $1.0 \mathrm{E}-07$ & $-1.1 \mathrm{E}-07$ & 7.4E-09 & $2.3 \mathrm{E}-07$ & 7.2E-08 & $-4.2 \mathrm{E}-08$ & $-5.7 \mathrm{E}-08$ & $1.7 \mathrm{E}-08$ & $-1.0 \mathrm{E}-07$ & $-2.8 \mathrm{E}-08$ \\
\hline & $\mathbf{A}_{\mathrm{zz}}$ & $8.0 \mathrm{E}-08$ & $-7.5 \mathrm{E}-08$ & $-2.6 \mathrm{E}-08$ & $7.2 \mathrm{E}-08$ & $1.1 \mathrm{E}-07$ & $-6.0 \mathrm{E}-08$ & $-5.8 \mathrm{E}-08$ & $5.6 \mathrm{E}-08$ & $-6.9 \mathrm{E}-08$ & $-4.1 \mathrm{E}-08$ \\
\hline \multirow{5}{*}{$\frac{\nabla}{8}$} & $\mathbf{A}_{\mathbf{x y}}$ & $-2.4 \mathrm{E}-08$ & $5.7 \mathrm{E}-08$ & $4.4 \mathrm{E}-08$ & $-4.2 \mathrm{E}-08$ & $-6.0 \mathrm{E}-08$ & $1.3 \mathrm{E}-07$ & $6.3 \mathrm{E}-08$ & $-5.4 \mathrm{E}-08$ & $3.0 \mathrm{E}-08$ & $6.6 \mathrm{E}-09$ \\
\hline & $\mathbf{A}_{\mathbf{x z}}$ & $-9.6 \mathrm{E}-08$ & $2.6 \mathrm{E}-08$ & $1.1 \mathrm{E}-07$ & $-5.7 \mathrm{E}-08$ & $-5.8 \mathrm{E}-08$ & $6.3 \mathrm{E}-08$ & $3.3 \mathrm{E}-07$ & $-1.6 \mathrm{E}-07$ & $1.1 \mathrm{E}-07$ & $5.1 \mathrm{E}-09$ \\
\hline & $\mathbf{A}_{\mathbf{y y}}$ & 7.6E-08 & $-1.2 \mathrm{E}-08$ & $-6.0 \mathrm{E}-08$ & $1.7 \mathrm{E}-08$ & $5.6 \mathrm{E}-08$ & $-5.4 \mathrm{E}-08$ & $-1.6 \mathrm{E}-07$ & $1.4 \mathrm{E}-07$ & $-5.7 \mathrm{E}-08$ & $1.2 \mathrm{E}-08$ \\
\hline & $\mathbf{A}_{\mathbf{y z}}$ & $-1.1 \mathrm{E}-07$ & $5.5 \mathrm{E}-08$ & $3.2 \mathrm{E}-08$ & $-1.0 \mathrm{E}-07$ & $-6.9 \mathrm{E}-08$ & $3.0 \mathrm{E}-08$ & $1.1 \mathrm{E}-07$ & $-5.7 \mathrm{E}-08$ & $1.5 \mathrm{E}-07$ & $6.1 \mathrm{E}-08$ \\
\hline & $\mathbf{A}_{\mathrm{zz}}$ & $-3.9 \mathrm{E}-08$ & $4.3 \mathrm{E}-08$ & $1.2 \mathrm{E}-08$ & $-2.8 \mathrm{E}-08$ & $-4.1 \mathrm{E}-08$ & $6.6 \mathrm{E}-09$ & $5.1 \mathrm{E}-09$ & $1.2 \mathrm{E}-08$ & $6.1 \mathrm{E}-08$ & $6.8 \mathrm{E}-08$ \\
\hline
\end{tabular}

Figure A.19: Multiple alignment tensor fit: variance-covariance matrix of the alignment tensor parameters of the best-fitting configuration $S S S$, calculated from Equation 6.1. The variance of each tensor data is depicted in red. 


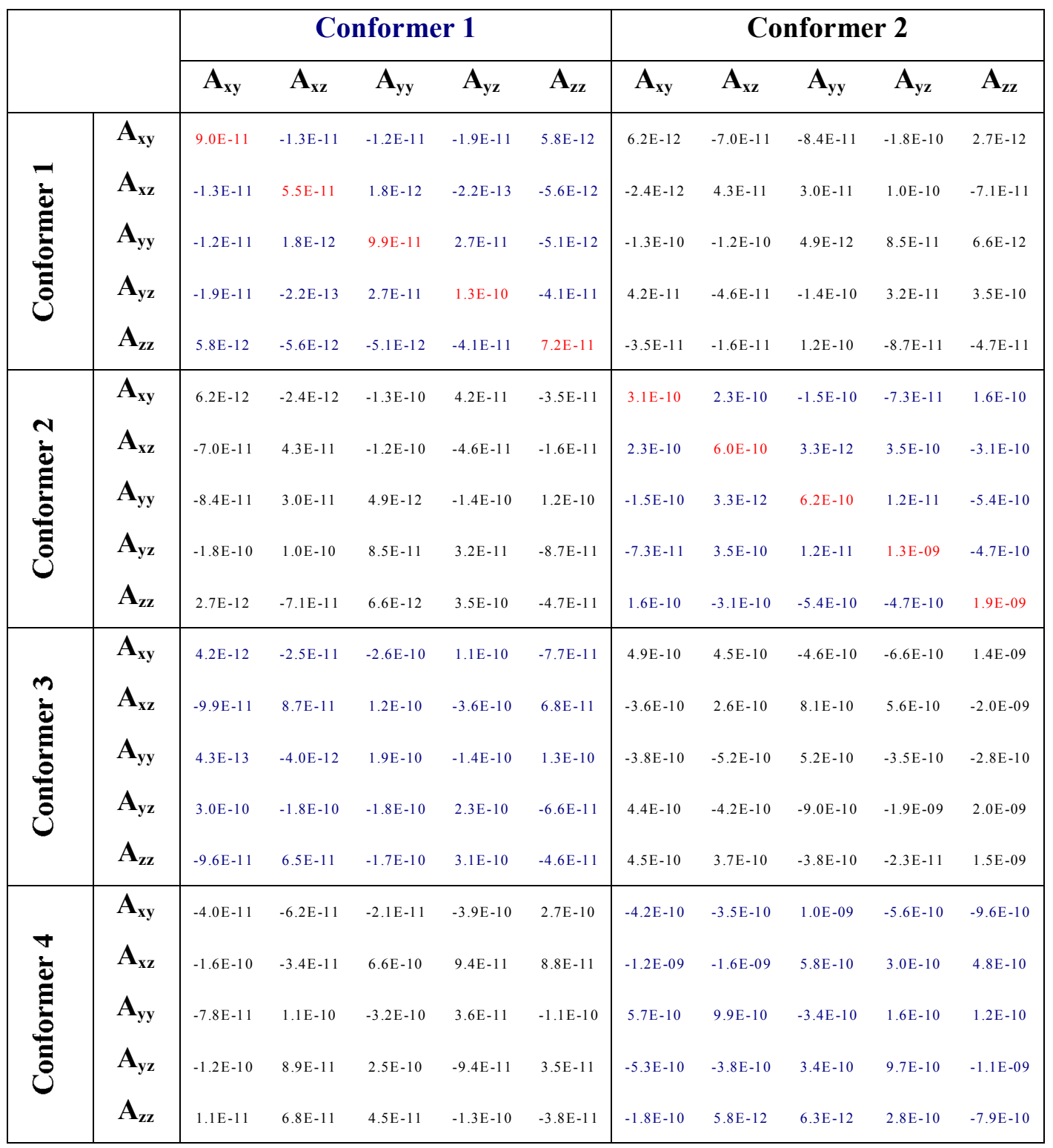

Figure A.20: Multiple alignment tensor fit: averaged variance-covariance matrix of the alignment tensor parameters of the best-fitting configuration $S S S$, calculated from Equation 6.2. The variance of each tensor data is depicted in red. 


\begin{tabular}{|c|c|c|c|c|c|c|c|c|c|c|c|}
\hline & & \multicolumn{5}{|c|}{ Conformer 3} & \multicolumn{5}{|c|}{ Conformer 4} \\
\hline & & $\mathbf{A}_{x y}$ & $\mathbf{A}_{\mathrm{xz}}$ & $\mathbf{A}_{\mathbf{y y}}$ & $\mathbf{A}_{\mathbf{y z}}$ & $\mathbf{A}_{\mathrm{zz}}$ & $\mathbf{A}_{\mathrm{xy}}$ & $\mathbf{A}_{\mathrm{xz}}$ & $\mathbf{A}_{\mathbf{y y}}$ & $\mathbf{A}_{\mathrm{yz}}$ & $\mathbf{A}_{\mathrm{zz}}$ \\
\hline \multirow{5}{*}{$\stackrel{\vec{\sigma}}{\underline{\theta}}$} & $\mathbf{A}_{\mathbf{x y}}$ & $4.2 \mathrm{E}-12$ & $-9.9 \mathrm{E}-11$ & $4.3 \mathrm{E}-13$ & $3.0 \mathrm{E}-10$ & $-9.6 \mathrm{E}-11$ & $-4.0 \mathrm{E}-11$ & $-1.6 \mathrm{E}-10$ & $-7.8 \mathrm{E}-11$ & $-1.2 \mathrm{E}-10$ & $1.1 \mathrm{E}-11$ \\
\hline & $\mathbf{A}_{\mathbf{x z}}$ & $-2.5 \mathrm{E}-11$ & $8.7 \mathrm{E}-11$ & $-4.0 \mathrm{E}-12$ & $-1.8 \mathrm{E}-10$ & $6.5 \mathrm{E}-11$ & $-6.2 \mathrm{E}-11$ & $-3.4 \mathrm{E}-11$ & $1.1 \mathrm{E}-10$ & $8.9 \mathrm{E}-11$ & $6.8 \mathrm{E}-11$ \\
\hline & $\mathbf{A}_{\mathbf{y y}}$ & $-2.6 \mathrm{E}-10$ & $1.2 \mathrm{E}-10$ & $1.9 \mathrm{E}-10$ & $-1.8 \mathrm{E}-10$ & $-1.7 \mathrm{E}-10$ & $-2.1 \mathrm{E}-11$ & $6.6 \mathrm{E}-10$ & $-3.2 \mathrm{E}-10$ & $2.5 \mathrm{E}-10$ & $4.5 \mathrm{E}-11$ \\
\hline & $\mathbf{A}_{\mathbf{y z}}$ & $1.1 \mathrm{E}-10$ & $-3.6 \mathrm{E}-10$ & $-1.4 \mathrm{E}-10$ & $2.3 \mathrm{E}-10$ & $3.1 \mathrm{E}-10$ & $-3.9 \mathrm{E}-10$ & $9.4 \mathrm{E}-11$ & $3.6 \mathrm{E}-11$ & $-9.4 \mathrm{E}-11$ & $-1.3 \mathrm{E}-10$ \\
\hline & $\mathbf{A}_{\mathrm{zz}}$ & $-7.7 \mathrm{E}-11$ & $6.8 \mathrm{E}-11$ & $1.3 \mathrm{E}-10$ & $-6.6 \mathrm{E}-11$ & $-4.6 \mathrm{E}-11$ & $2.7 \mathrm{E}-10$ & $8.8 \mathrm{E}-11$ & $-1.1 \mathrm{E}-10$ & $3.5 \mathrm{E}-11$ & $-3.8 \mathrm{E}-11$ \\
\hline \multirow{5}{*}{$\frac{N}{0}$} & $\mathbf{A}_{\mathbf{x y}}$ & $4.9 \mathrm{E}-10$ & $-3.6 \mathrm{E}-10$ & $-3.8 \mathrm{E}-10$ & $4.4 \mathrm{E}-10$ & $4.5 \mathrm{E}-10$ & $-4.2 \mathrm{E}-10$ & $-1.2 \mathrm{E}-09$ & $5.7 \mathrm{E}-10$ & $-5.3 \mathrm{E}-10$ & $-1.8 \mathrm{E}-10$ \\
\hline & $\mathbf{A}_{\mathrm{xz}}$ & $4.5 \mathrm{E}-10$ & $2.6 \mathrm{E}-10$ & $-5.2 \mathrm{E}-10$ & $-4.2 \mathrm{E}-10$ & $3.7 \mathrm{E}-10$ & $-3.5 \mathrm{E}-10$ & $-1.6 \mathrm{E}-09$ & $9.9 \mathrm{E}-10$ & $-3.8 \mathrm{E}-10$ & $5.8 \mathrm{E}-12$ \\
\hline & $\mathbf{A}_{\mathbf{y y}}$ & $-4.6 \mathrm{E}-10$ & $8.1 \mathrm{E}-10$ & $5.2 \mathrm{E}-10$ & $-9.0 \mathrm{E}-10$ & $-3.8 \mathrm{E}-10$ & $1.0 \mathrm{E}-09$ & $5.8 \mathrm{E}-10$ & $-3.4 \mathrm{E}-10$ & $3.4 \mathrm{E}-10$ & $6.3 \mathrm{E}-12$ \\
\hline & $\mathbf{A}_{\mathbf{y z}}$ & $-6.6 \mathrm{E}-10$ & $5.6 \mathrm{E}-10$ & $-3.5 \mathrm{E}-10$ & $-1.9 \mathrm{E}-09$ & $-2.3 \mathrm{E}-11$ & $-5.6 \mathrm{E}-10$ & $3.0 \mathrm{E}-10$ & $1.6 \mathrm{E}-10$ & $9.7 \mathrm{E}-10$ & $2.8 \mathrm{E}-10$ \\
\hline & $\mathbf{A}_{\mathrm{zz}}$ & $1.4 \mathrm{E}-09$ & $-2.0 \mathrm{E}-09$ & $-2.8 \mathrm{E}-10$ & $2.0 \mathrm{E}-09$ & $1.5 \mathrm{E}-09$ & $-9.6 \mathrm{E}-10$ & $4.8 \mathrm{E}-10$ & $1.2 \mathrm{E}-10$ & $-1.1 \mathrm{E}-09$ & $-7.9 \mathrm{E}-10$ \\
\hline \multirow{5}{*}{ 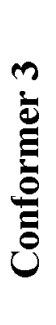 } & $\mathbf{A}_{\mathbf{x y}}$ & $3.3 \mathrm{E}-09$ & $-1.5 \mathrm{E}-09$ & $-8.4 \mathrm{E}-10$ & $2.3 \mathrm{E}-09$ & $1.8 \mathrm{E}-09$ & $-5.4 \mathrm{E}-10$ & $-2.2 \mathrm{E}-09$ & $1.7 \mathrm{E}-09$ & $-2.5 \mathrm{E}-09$ & $-8.8 \mathrm{E}-10$ \\
\hline & $\mathbf{A}_{\mathbf{x z}}$ & $-1.5 \mathrm{E}-09$ & $2.7 \mathrm{E}-09$ & $9.3 \mathrm{E}-10$ & $-2.5 \mathrm{E}-09$ & $-1.7 \mathrm{E}-09$ & $1.3 \mathrm{E}-09$ & $5.8 \mathrm{E}-10$ & $-2.8 \mathrm{E}-10$ & $1.2 \mathrm{E}-09$ & $9.8 \mathrm{E}-10$ \\
\hline & $\mathbf{A}_{\mathbf{y y}}$ & $-8.4 \mathrm{E}-10$ & $9.3 \mathrm{E}-10$ & $1.9 \mathrm{E}-09$ & $1.7 \mathrm{E}-10$ & $-6.0 \mathrm{E}-10$ & $9.9 \mathrm{E}-10$ & $2.4 \mathrm{E}-09$ & $-1.4 \mathrm{E}-09$ & 7.3E-10 & $2.8 \mathrm{E}-10$ \\
\hline & $\mathbf{A}_{\mathbf{y z}}$ & $2.3 \mathrm{E}-09$ & $-2.5 \mathrm{E}-09$ & $1.7 \mathrm{E}-10$ & $5.3 \mathrm{E}-09$ & $1.6 \mathrm{E}-09$ & $-9.5 \mathrm{E}-10$ & $-1.3 \mathrm{E}-09$ & $4.0 \mathrm{E}-10$ & $-2.3 \mathrm{E}-09$ & $-6.3 \mathrm{E}-10$ \\
\hline & $\mathbf{A}_{\mathrm{zz}}$ & $1.8 \mathrm{E}-09$ & $-1.7 \mathrm{E}-09$ & $-6.0 \mathrm{E}-10$ & $1.6 \mathrm{E}-09$ & $2.5 \mathrm{E}-09$ & $-1.4 \mathrm{E}-09$ & $-1.3 \mathrm{E}-09$ & $1.3 \mathrm{E}-09$ & $-1.6 \mathrm{E}-09$ & $-9.3 \mathrm{E}-10$ \\
\hline \multirow{5}{*}{ 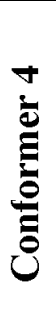 } & $\mathbf{A}_{\mathbf{x y}}$ & $-5.4 \mathrm{E}-10$ & $1.3 \mathrm{E}-09$ & $9.9 \mathrm{E}-10$ & $-9.5 \mathrm{E}-10$ & $-1.4 \mathrm{E}-09$ & $3.0 \mathrm{E}-09$ & $1.4 \mathrm{E}-09$ & $-1.2 \mathrm{E}-09$ & $6.8 \mathrm{E}-10$ & $1.5 \mathrm{E}-10$ \\
\hline & $\mathbf{A}_{\mathbf{x z}}$ & $-2.2 \mathrm{E}-09$ & $5.8 \mathrm{E}-10$ & $2.4 \mathrm{E}-09$ & $-1.3 \mathrm{E}-09$ & $-1.3 \mathrm{E}-09$ & $1.4 \mathrm{E}-09$ & $7.5 \mathrm{E}-09$ & $-3.6 \mathrm{E}-09$ & $2.5 \mathrm{E}-09$ & $1.2 \mathrm{E}-10$ \\
\hline & $\mathbf{A}_{\mathbf{y y}}$ & $1.7 \mathrm{E}-09$ & $-2.8 \mathrm{E}-10$ & $-1.4 \mathrm{E}-09$ & $4.0 \mathrm{E}-10$ & $1.3 \mathrm{E}-09$ & $-1.2 \mathrm{E}-09$ & $-3.6 \mathrm{E}-09$ & $3.2 \mathrm{E}-09$ & $-1.3 \mathrm{E}-09$ & $2.7 \mathrm{E}-10$ \\
\hline & $\mathbf{A}_{\mathbf{y z}}$ & $-2.5 \mathrm{E}-09$ & $1.2 \mathrm{E}-09$ & $7.3 \mathrm{E}-10$ & $-2.3 \mathrm{E}-09$ & $-1.6 \mathrm{E}-09$ & $6.8 \mathrm{E}-10$ & $2.5 \mathrm{E}-09$ & $-1.3 \mathrm{E}-09$ & $3.4 \mathrm{E}-09$ & $1.4 \mathrm{E}-09$ \\
\hline & $\mathbf{A}_{\mathrm{zz}}$ & $-8.8 \mathrm{E}-10$ & $9.8 \mathrm{E}-10$ & $2.8 \mathrm{E}-10$ & $-6.3 \mathrm{E}-10$ & $-9.3 \mathrm{E}-10$ & $1.5 \mathrm{E}-10$ & $1.2 \mathrm{E}-10$ & $2.7 \mathrm{E}-10$ & $1.4 \mathrm{E}-09$ & $1.5 \mathrm{E}-09$ \\
\hline
\end{tabular}

Figure A.21: Multiple alignment tensor fit: veraged variance-covariance matrix of the alignment tensor parameters of the best-fitting configuration $S S S$, calculated from Equation 6.2. The variance of each tensor data is depicted in red. 


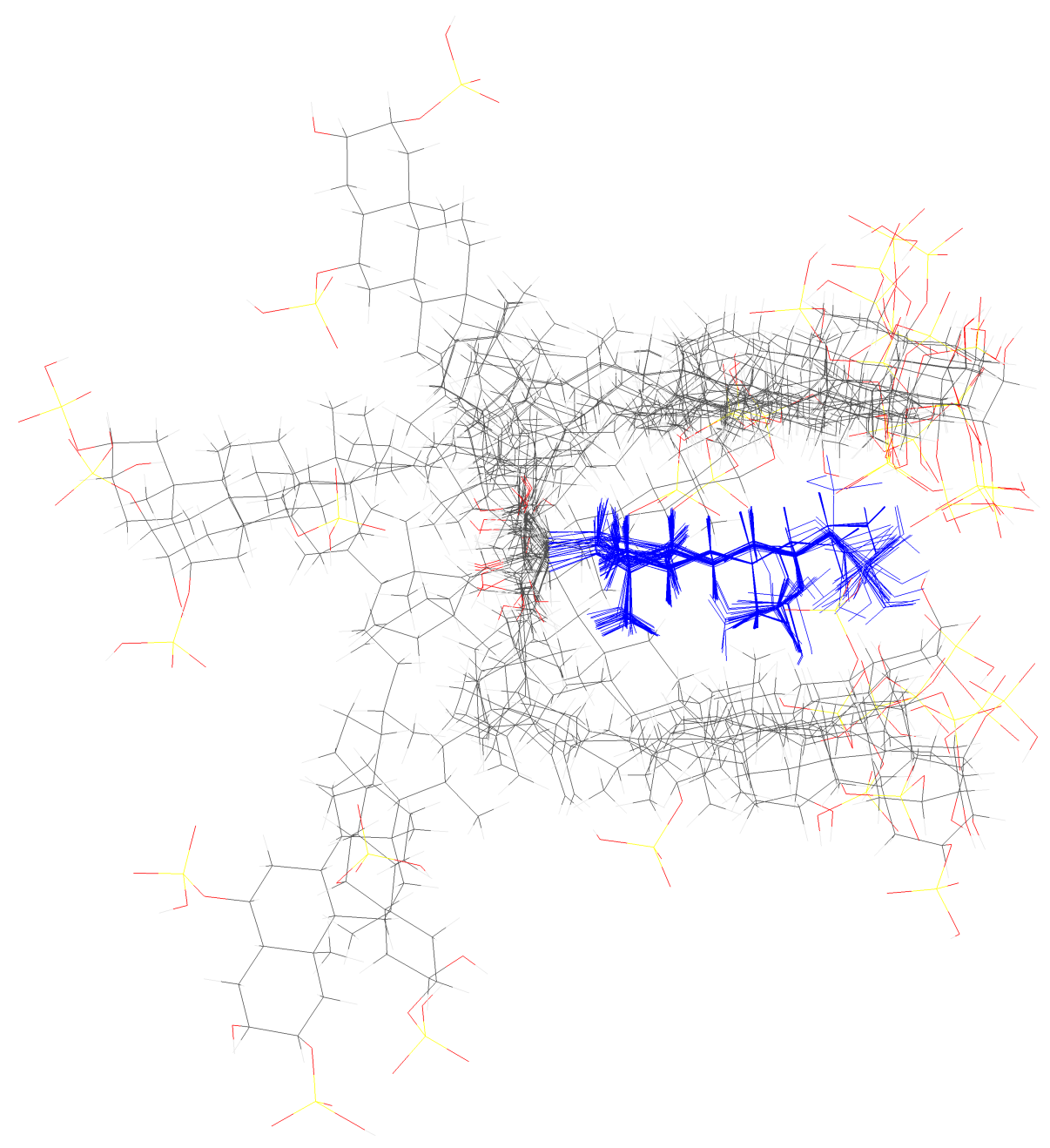

Figure A.22: Single alignment tensor fit: the remaining structures of configuration $S S S$ after the filtering step using ${ }^{3} J_{\mathrm{HH}}$ couplings, superimposed on steroid ABCD, which is visualized in blue. 
Experimental RDCs versus back-calculated RDCs with an ensemble number of 2

(a)

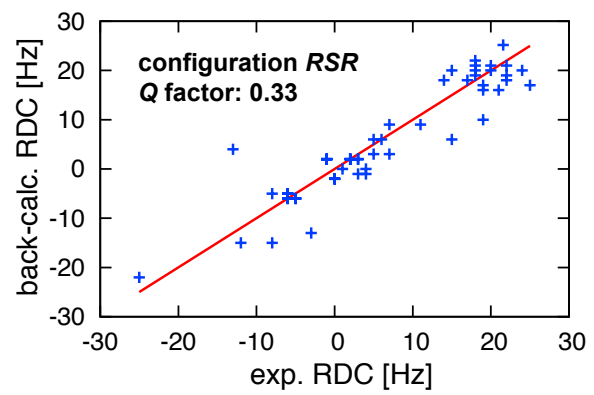

Experimental RDCs versus back-calculated RDCs with an ensemble number of 2

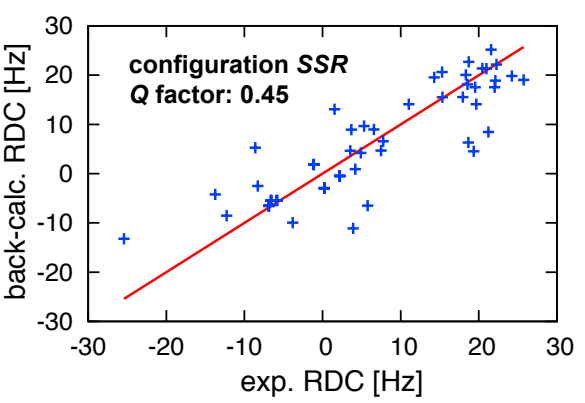

Experimental RDCs versus back-calculated RDCs with an ensemble number of 3

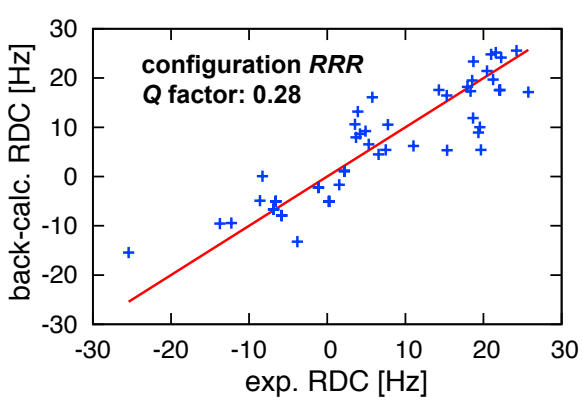

Experimental RDCs versus back-calculated RDCs with an ensemble number of 3

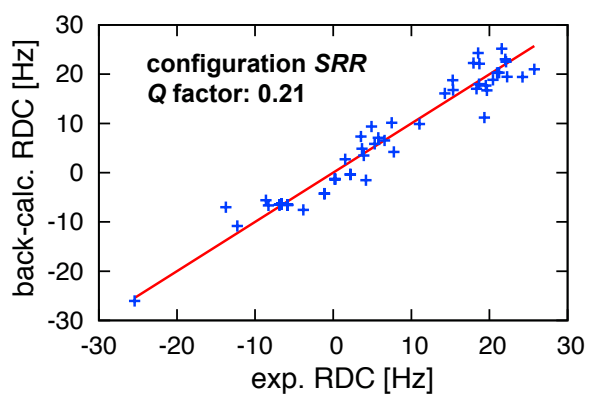

Experimental RDCs versus back-calculated RDCs with an ensemble number of 2

(b)

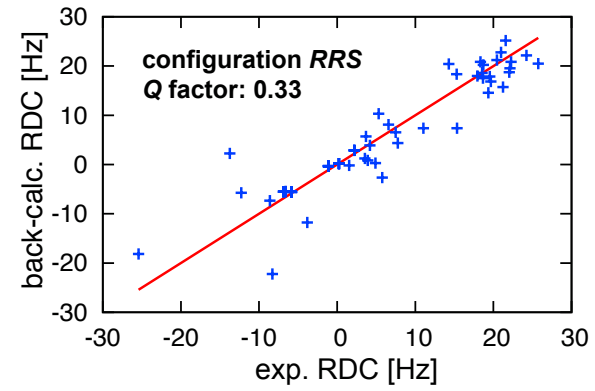

Experimental RDCs versus back-calculated RDCs with an ensemble number of 3

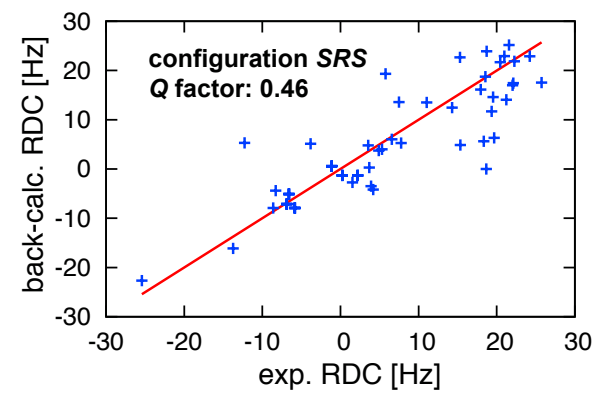

Experimental RDCs versus back-calculated RDCs with an ensemble number of 3

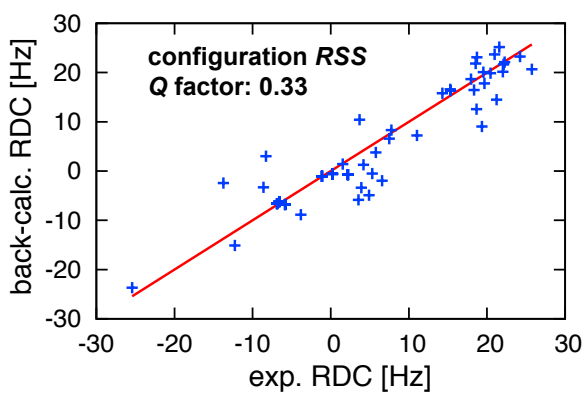

Experimental RDCs versus back-calculated RDCs with an ensemble number of 3

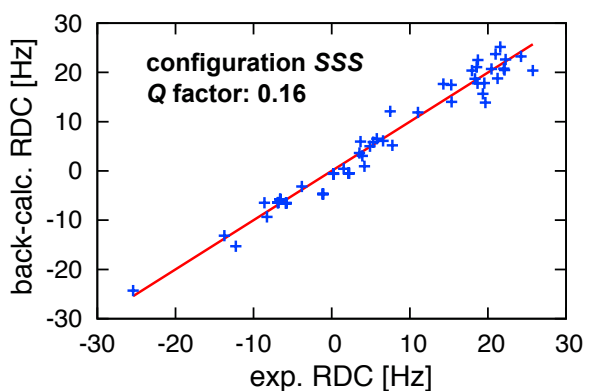

Figure A.23: Single alignment tensor fit: comparison of the correlations of experimental and back-calculated RDCs of 8 possible configurations. 


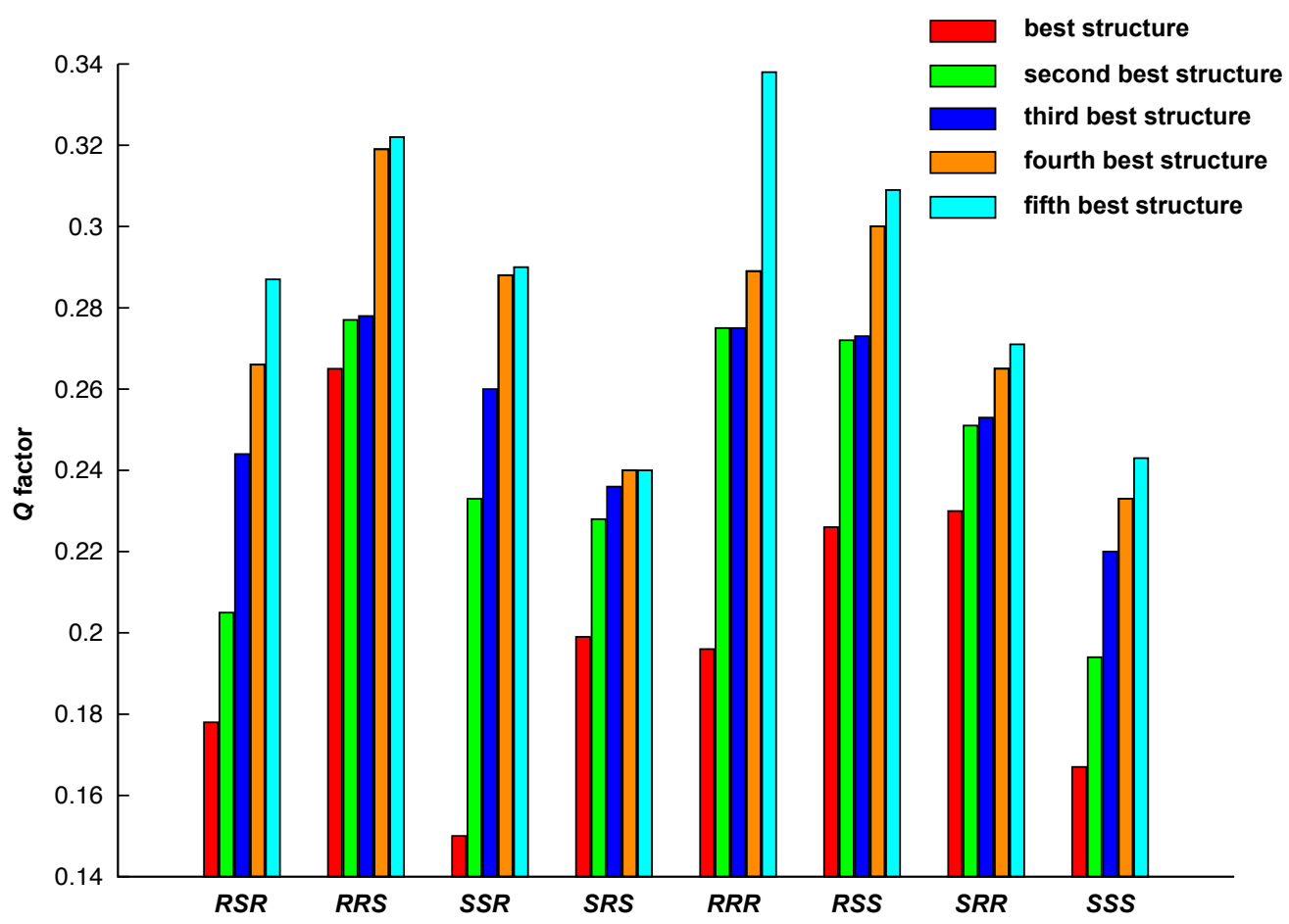

Figure A.24: Single alignment tensor fit: Comparison of the $Q$ factors of the RDC fitting of the five best structures between 8 configurations. The RDC fitting was performed on a subgroup of experimental RDCs, which includes the data from one steroid A'B'C'D' and the linker region.

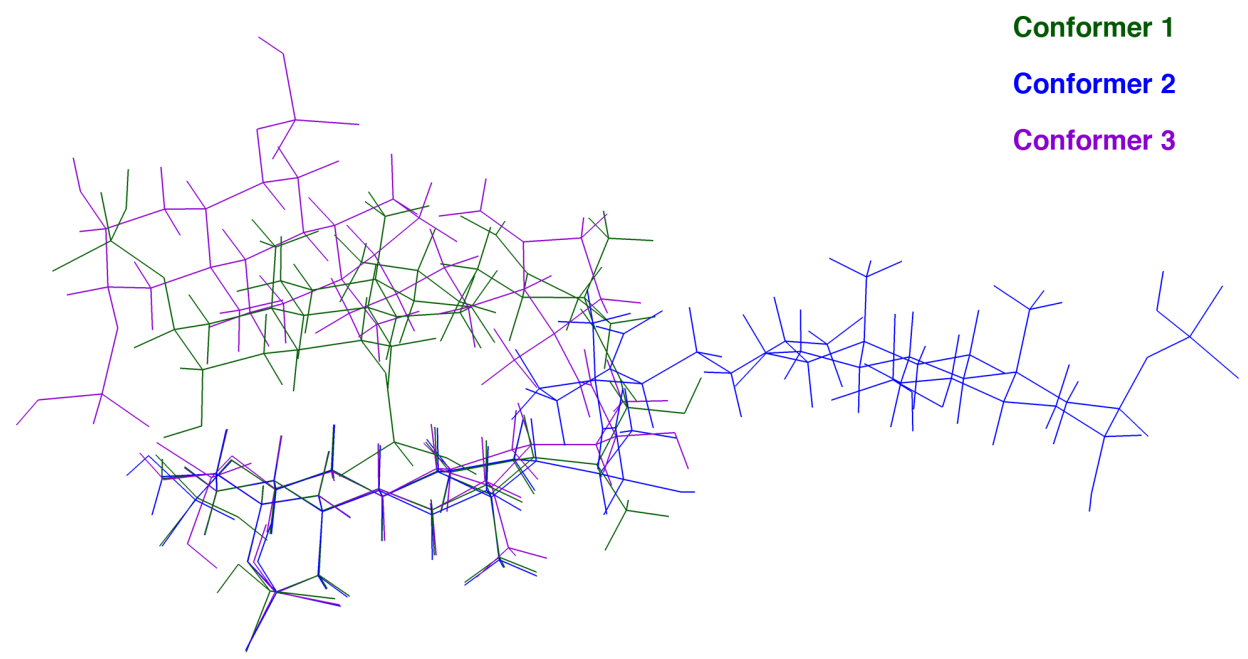

Figure A.25: Single alignment tensor fit: Three populated conformations of the bestfitting configuration $S S S$, superimposed on the steroid A'B'C'D'. The SVD fitting of the 44 ${ }^{1} D_{\mathrm{CH}} \mathrm{RDCs}$ was performed against an ensemble of five structures, which are superimposed to the steroid A'B'C'D'. One alignment tensor for all structures within one ensemble is assumed. 


\section{Appendix B}

\section{Additional equations for thermodynamics}

In Gaussian the thermodynamic quantities of the molecules are calculated as follows:

$$
\begin{aligned}
E & =E_{0}+E_{\text {vib }}+E_{\text {rot }}+E_{\text {trans }} \\
H & =E+R T \\
G & =H-T S
\end{aligned}
$$

where $E, H$ and $G$ are the thermal energy, enthalpy, and free energy (free enthalpy), respectively. The thermal energy $E$ and entropy $S$ are calculated from the partition function $q$ with the following relation:

$$
\begin{aligned}
E & =N k_{B} T^{2}\left(\frac{\partial \ln q}{\partial T}\right)_{V} \\
S & =R\left(\ln \left(q_{t} q_{e} q_{r} q_{v} e\right)+T\left(\frac{\partial \ln q}{\partial T}\right)_{V}\right) .
\end{aligned}
$$

The translational partition function is calculated in Gaussian as:

$$
q_{t}=\left(\frac{2 \pi m k_{B} T}{h^{2}}\right)^{3 / 2} \frac{K_{B} T}{P} .
$$

Therefore the contribution from the translation to the internal thermal energy and entropy are: 


$$
\begin{aligned}
E_{t} & =\frac{3}{2} R T, \\
S_{t} & =R\left(\ln q_{t}+\frac{5}{2}\right) .
\end{aligned}
$$

Using the assumption in Gaussian that the first electronic excitation energy is much greater than $k_{B} T$, the electronic partition function is:

$$
q_{e}=\omega_{0},
$$

where $\omega_{0}$ is the degeneracy grade of the $0^{\text {th }}$ level. Using this the entropy due to electronic motion is calculated as:

$$
S_{e}=R \ln q_{e}
$$

Since there are no temperature dependent terms in the partition function, the electronic contribution to the internal thermal energy is zero.

For a nonlinear polyatomic molecule, the rotational partition function is:

$$
q_{r}=\frac{\pi^{1 / 2}}{\sigma_{r}}\left(\frac{T^{3 / 2}}{\left(\Theta_{r, x} \Theta_{r, y} \Theta_{r, z}\right)^{1 / 2}}\right) .
$$

where $\Theta_{r}$ is the moment of inertia:

$$
\Theta=\frac{h^{2}}{8 \pi^{2} I k_{B}}
$$

Using this partition function the contribution from the rotation to the internal thermal energy and entropy are calculated as:

$$
\begin{array}{r}
E_{r}=\frac{3}{2} R T, \\
S_{r}=R\left(\ln q_{r}+\frac{3}{2}\right) .
\end{array}
$$

In Gaussian the zero-point energy is computed separately, therefore the partition function for each vibrational level is calculated as:

$$
q_{v, K}=\frac{1}{1-e^{-\Theta_{v, K} / T}}
$$


and the overall vibrational partition function is:

$$
q_{v}=\prod_{K} \frac{1}{1-e^{-\Theta_{v, K} / T}} .
$$

where $\Theta_{v, K}$ is the characteristic vibrational temperature. It is given by:

$$
\Theta_{v, K}=\frac{h \nu_{K}}{k_{B}}
$$

where $\nu_{K}$ is the vibrational frequency. The total contribution to internal thermal energy and entropy from the vibrational partition function are:

$$
\begin{aligned}
E_{v} & =R \sum_{K} \Theta_{v, K}\left(\frac{1}{2}+\frac{1}{e^{\Theta_{v, K} / T}-1}\right), \\
S_{v} & =R \sum_{K}\left(\frac{\Theta_{v, K} / T}{e^{\Theta_{v, K} / T}-1}-\ln \left(1-e^{-\Theta_{v, K} / T}\right)\right) .
\end{aligned}
$$


198 APPENDIX B. ADDITIONAL EQUATIONS FOR THERMODYNAMICS 


\section{Appendix C}

\section{Pulse program}

For the RDC measurements a CLIP-HSQC pulse program has been implemented into Bruker format according to [Enthart et al., 2008]. The sequence is given as follows.

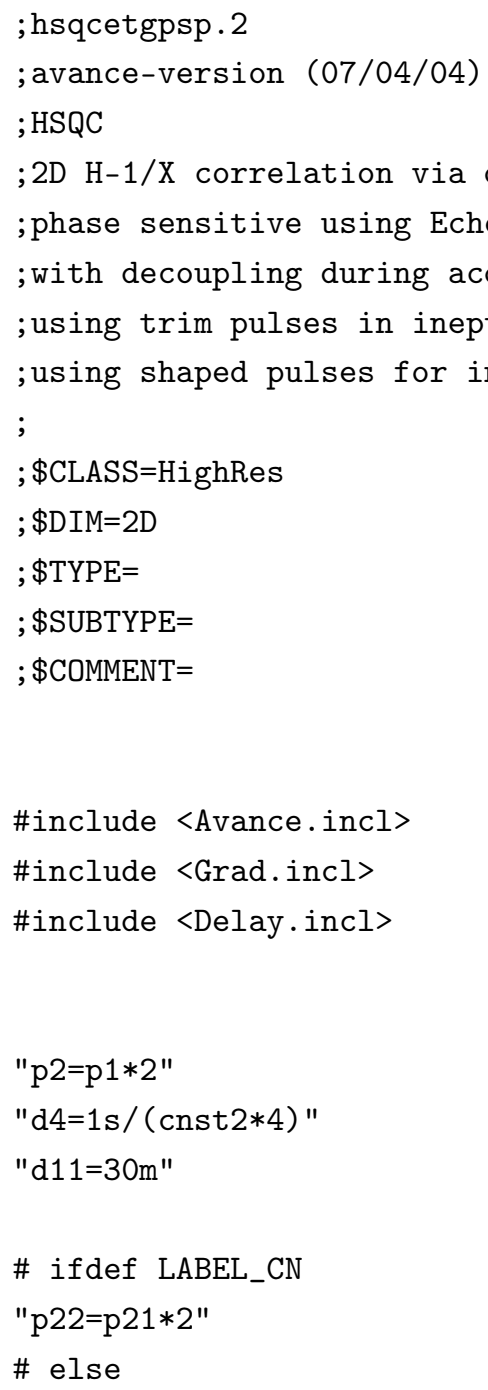


\# endif / $*$ LABEL_CN*/

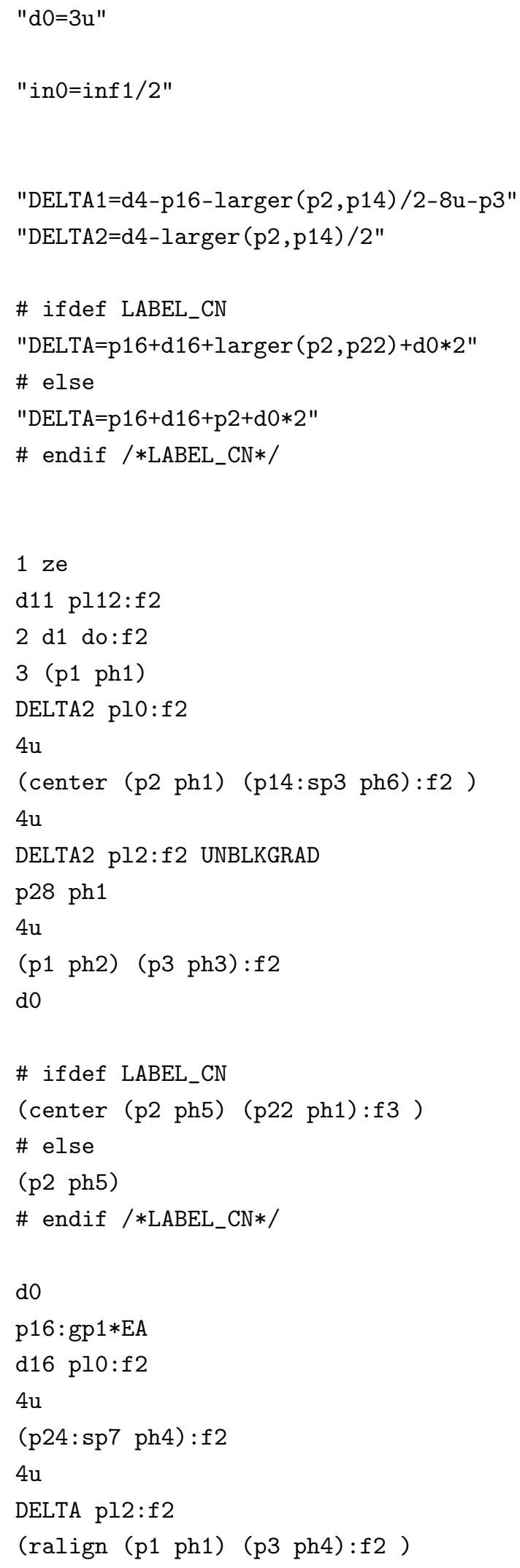




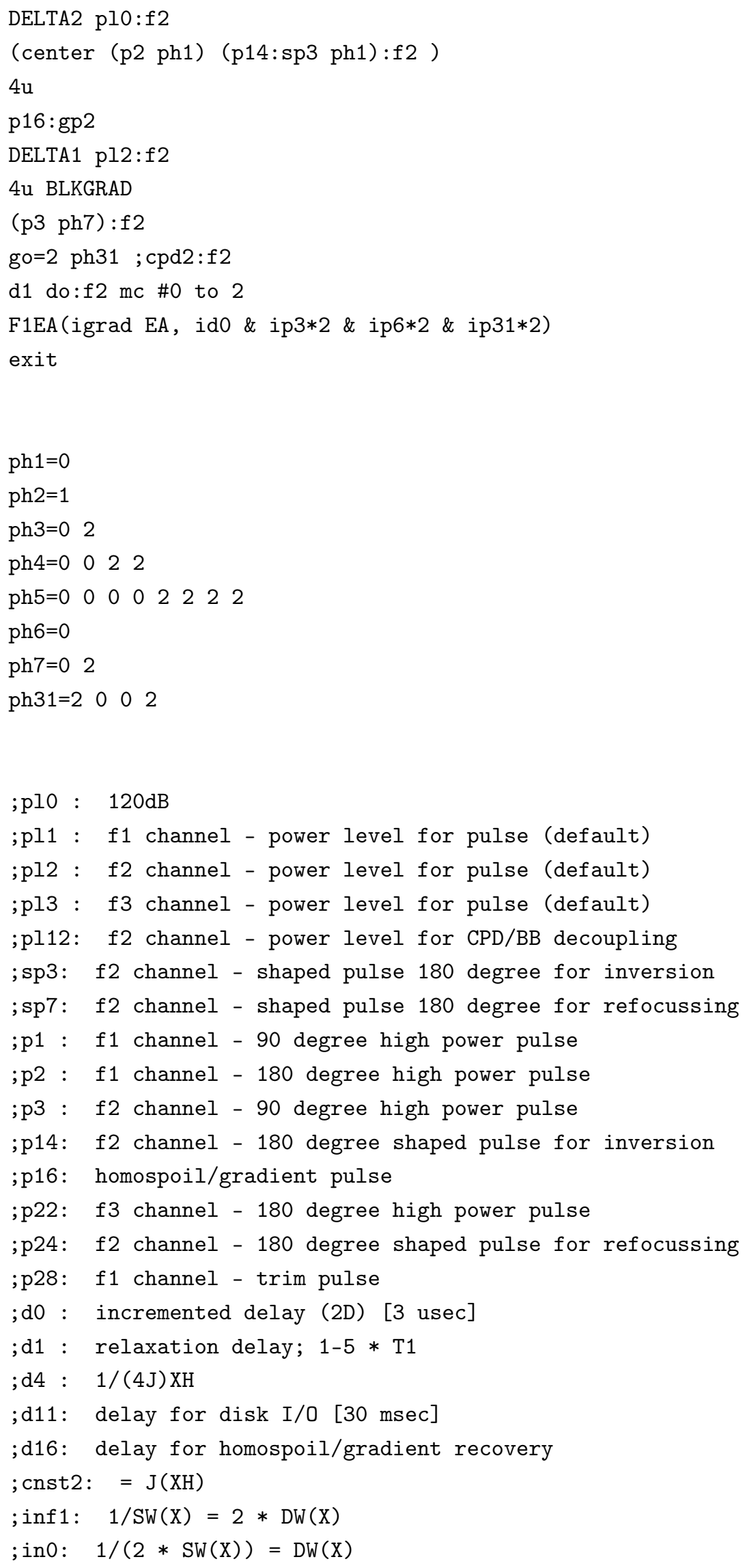


;ndo: 2

;NS: $1 * \mathrm{n}$

;DS: $>=16$

;td1: number of experiments

;FnMODE: echo-antiecho

; cpd2: decoupling according to sequence defined by cpdprg2

;pcpd2: f2 channel - 90 degree pulse for decoupling sequence

; use gradient ratio: gp 1 : gp 2

; 80 : 20.1 for $\mathrm{C}-13$

; 80 : 8.1 for $\mathrm{N}-15$

;for z-only gradients:

;gpz1: $80 \%$

;gpz2: $20.1 \%$ for $\mathrm{C}-13,8.1 \%$ for $\mathrm{N}-15$

; use gradient files:

;gpnam1: SINE.100

;gpnam2: SINE.100

; preprocessor-flags-start

;LABEL_CN: for C-13 and N-15 labeled samples start experiment with

; option -DLABEL_CN (eda: ZGOPTNS)

; preprocessor-flags-end

;\$Id: hsqcetgpsp.2,v 1.5 2007/04/11 13:34:30 ber Exp \$ 


\section{Lebenslauf}

\section{Persönliche Daten}

$\begin{array}{ll}\text { Name } & \text { Han Sun } \\ \text { Geburtstag } & 07.08 .1984 \\ \text { Geburtsort } & \text { Peking, V. R. China } \\ \text { Staatsangehörigkeit } & \text { V. R. China }\end{array}$

\section{Ausbildung}

$03 / 2009$

Doktorandin in der Abteilung NMR-basierte Strukturbiologie am Max-Planck-Institut für biophysikalische Chemie in Göttingen; Titel der Arbeit unter Anleitung von Prof. Dr. Christian Griesinger: Stereochemistry of Challenging Natural Products Studied by NMR-based Methods

$01 / 09$

$03 / 2008-12 / 2008$

$10 / 2004-01 / 2009$

09/2003-08/2004

$09 / 2000-07 / 2003$

Abschluss als Diplom Chemikerin

Diplomarbeit in der Abteilung NMR-basierte Strukturbiologie am Max-Planck-Institut für biophysikalische Chemie in Göttingen; Titel der Arbeit unter Anleitung von Prof. Dr. Christian Griesinger: Konformations- und Konfigurationsbestimmung von Canescensterone und einem Evans Produkt mittels isotroper and anisotroper NMR-Parameter

Studium der Chemie an der Georg-August-Universität Göttingen, Göttingen, Deutschland

Studium der Chemie an der Nankai Universität, Nankai, V. R. China

Gymnasium der Tsinghua Universität, Peking, V. R. China

$09 / 1997-07 / 2000$

Peking Nr. 41 Mittelschule, Peking, V. R. China

\section{Auslandsaufenthalt}

$10 / 2009-11 / 2009$

Wissenschaftliche Mitarbeiterin bei Dr. Armando NavarroVázquez, Universität Vigo, Vigo, Spanien 